\title{
Les productions de l'atelier de la Muette
}

\author{
Martine Genin, Armand Desbat, Sandrine Elaigne, Colette Laroche \\ et Bernard DANGRÉAUX
}

\section{MÉTHODES D'INVENTAIRE ET D'ÉTUDE}

I.es productions sigillées représentent un fonds documentaire dont l'importance quantitative et l'état de fragmentation ont imposé certains choix dans les méthodes d'inventaire et d'étude.

En premier lieu, un recollage sur l'ensemble destiné à déterminer un nombre minimum de vases selon la Góméthode Arcelin-Pradelle, aujourd'hui couramment titomployée, a été exclu. Cette méthode qui a fait ses preuves et que nous utilisons à Lyon dans l'étude de mobilier stratifié et d'ensembles clos ne paraissait pas convenir ou se justifier pour l'inventaire et l'étude de proEriuctions d'atclicr. Nous avons considéré qu'effectuer des ocomptages systématiques sur la totalité des fragments ¿n'apporterait en effet rien de significatif tout en représentant une énorme perte de temps. Un premier travail a donc consisté à isoler les éléments identifiables, rebords et fonds, puis à classer les bords par groupes morphologiques (plats, assiettes, bols) et typologiques (service I, service II, divers) afin d'évaluer les fréquences respectives de ces groupes et types.

\section{MISE EN PLACE DU CADRE DE L'ÉTUDE}

\section{JUSTIFICATION DU CHOIX DES RÉFÉRENCES TYPOLOGIQUES}

L'étude des productions sigillées de l'atelier nous a logiquement amenés à réfléchir sur la façon dont il fallait présenter le matériel : devions-nous bâtir de toutes pièces une nouvelle typologie propre à ce seul atelier, continuer de nous référer à des classifications existantes mais déjà anciennes et parfois contestables, ou bien encore utiliser le Conspectus, typologie la plus récente dont l'usage tend à se généraliser aujourd'hui?

Le travail effectué sur les productions de la Muette nous a assez vite montré que la typologie présentée dans le Conspectus ne pouvait guère être utilisée dans l'identification, l'étude et la présentation des sigillées lyonnaises. On observe en effet que les auteurs font table rase des classifications antérieures, et en premier lieu pour ce qui nous concerne, de celle que $S$. Loeschcke a établie en 1909 d'après la sigillée du camp de Haltern : ils tentent de justifier cette démarche en arguant du fait que ce classement est insuffisant et largement inadéquat.

La classification de S. Loeschcke en quatre services s'avère effectivement en partie inadéquate, et nous avons déjà eu l'occasion de la remettre en question, notamment pour les services III et IV (Genin, 1993). En revanche, l'abandon des services I et II, et à l'intérieur de chacun d'entre eux des séries A, B, C, ne semble pas se justifier, en particulier à Lyon, et cela même si on nuance ou si on conteste dans le détail les implications chronologiques de cet ancien classement. À cet égard, les exemples des formes 12 et 14 du Conspectus sont particulièrement significatifs : ces deux formes rassemblent respectivement les assiettes et les bols du « service I " au sens large, en mélangeant des séries morphologiques distinctes (IB, IC) et en présentant sur le même plan des variantes précoces, peu standardisées, des profils classiques ou bien encore des formes dont on sait aujourd'hui qu'elles furent sans doute produites exclusivement à Lyon.

À quoi servent de tels regroupements? En quoi sont-ils censés faciliter l'identification de ces assiettes et de ces bols pour les non-spécialistes? Ne rendent-ils pas au contraire encore plus ardue l'appréhension du matériel comme production d'un atelier donné à une époque donnée obéissant à un programme précis et inscrit dans une logique commerciale? Il nous semble que la tentative 
de clarification annoncée aboutit en fait à brouiller les cartes en nivelant l'ensemble des problèmes, sinon en les niant, par le biais de regroupements typologiques parfois très larges dont la validité n'est d'ailleurs pas toujours prouvée (voir également, pour exemple, les formes 1 à 5). Par ailleurs, les travaux menés par S. von Schnurbein et M. Picon sur la sigillée de Haltern ont montré l'importance quantitative des productions lyonnaises au sein du matériel, et abandonner totalement la classification de Loeschcke dans l'étude des sigillées de la Muette n'apparaît pas comme une démarche cohérente dans le cadre de la recherche que nous avons engagée.

Enfin, l'usage d'anciennes classifications, entériné par le temps et l'habitude, ne semble pas faire obstacle à la réflexion et à la recherche; autrement dit, se référer à $S$. Loeschcke ou, plus près de nous, à ('hr. Goudineau, ne signifie pas que nous ne prenons pas en compte les progrès des connaissances depuis la parution de ces travaux ou qu'on s'interdit, le cas échéant, de proposer des rectifications en apportant des informations supplémentaires, qu'elles soient d'ordre chronologique ou typologique.

De façon plus générale, les difficultés que l'on éprouve à utiliser la nouvelle typologie du Conspectus tiennent à quelques grands principes de base adoptés par les auteurs et que l'étude des productions lyonnaises permet aujourd'hui de remettre globalement en question.

Le premier principe est qu'on ne peut, en l'absence d'analyses physico-chimiques de pâtes, distinguer les différentes productions sigillées des ateliers italiques ou de leurs succursales gauloises. De là, toutes ces productions se retrouvent donc dans un pot commun appelé «sigillée de type italique".

Parallèlement, les auteurs tiennent pour certaine la suprématie absolue d'Arezzo sur les autres ateliers italiques et adoptent donc un point de vue monolithique et linéaire ne pouvant déboucher que sur une typologie tronquée. Rappelons que les ateliers italiques sont sans doute plus nombreux qu'on ne le pensait jusqu'alors, et que de plus aucun d'entre eux n'a, à ce jour, fait l'objet d'études détaillées et encore moins de travaux de synthèse. Or, ce que nous savons aujourd'hui des productions sigillées de la Muette (chronologie, degrés de standardisation et composition du répertoire par période) incite à la prudence et montre la nécessité de ne pas séparer arbitrairement typologie, chronologie et lieu de fabrication. Une typologie doit effectivement constituer un système de classification d'une réalité donnée. Il s'agit en l'occurrence des productions de sigillée dans le monde romain, en commençant par les productions d'époque augustéenne. Or cette réalité est complexe et il ne semble pas rigoureux de s'appuyer sur des constats d'impuissance (difficulté ou impossibilité d'attribuer telle sigillée à tel centre de production) et sur notre ignorance (absence de monographies d'ateliers) au seuil d'un travail aussi ambitieux.

Le deuxième principe, discuté plus haut, consiste à vouloir remplacer toutes les typologies antérieures afin de constituer un canevas « universel » pouvant servir aussi bien aux céramologues qu'aux archéologues ou historiens ayant affaire avec le matériel de façon sporadique. Tout se passe donc comme si les répertoires des différents ateliers avaient peu ou prou connu un développement similaire à la même époque, ce qui reste à prouver, comme nous l'indiquions plus haut.

Le troisième principe stipule que seuls les profils complets permettant une identification fiable sont pris en compte; les fragments isolés ne sont donc pas, de façon délibérée, considérés comme des vases proprement dits. Ce parti pris conduit logiquement à oblitérer la partie du matériel dont on ne sait pas quoi faire, et dont on ne saura jamais quoi faire aussi longtemps qu'on refusera de l'examiner dans le détail et, à terme, de la classifier. Que saurait-on du matériel de Haltern ou de Bolsena si S. Loeschcke ou Chr. Goudineau avaient exclu les vases incomplets ou les fragments isolés?

Cette nouvelle classification apparaît donc comme un ensemble de tiroirs dans lesquels sont rangés, par forme, des vases qui se ressemblent sur un plan purement morphologique et typologique, et ce, quelles que soient leur provenance et leur datation. Or, une typologie qui porte uniquement sur les formes tend à devenir une simple collection d'objets ne traduisant aucune des réalités liées à la fabrication et à la diffusion de ces objets.

Il semble donc logique qu'au bout du compte elle s'apparente plus à un catalogue ou à une vitrine de musée qu'à un outil de travail utile pour la recherche céramologique.

Nous avons envisagé l'étude et la publication des productions lyonnaises sous un angle radicalement différent. Il s'agissait pour nous de caractériser ces productions afin de donner l'image la plus fidèle qui soit d'un répertoire donné, dont seule une analyse fine pouvait permettre de déboucher sur les questions que pose cet atelier de la Muette : sa chronologie, son rôle, son fonctionnement, soit l'organisation de la production et de la diffusion (problèmes liés aux notions de standardisation et de 
"spécialisation"). Notre ambition n'était donc pas de bâtir une typologie fermée propre à un seul atelier, et dans un souci de cohérence et de clarté, nous avons choisi de faire référence au classement de $\mathrm{S}$. Loeschcke, d'ailleurs repris en 1982 par S. von Schnurbein, qu il s'agisse des "services " I et II proprement dits et de leurs subdivisions ou des "types", beaucoup plus rarement représentés, qui leur sont associés.

\section{MODE DE PRÉSENTATION DU MATÉRIEL}

L'étude des formes montre la présence d'un grand nombre de variantes dans les différentes séries morphologiques du service I et du service II. Le classement par groupes finalement retenu ne prétend pas constituer une typologie fine et achevée : il correspond davantage à un outil destiné à faciliter une " lecture » simple et rapide du matériel et à établir des comparaisons extérieures.

Parallèlement, les vases sigillés ont fait l'objet d'un catalogue décrivant de façon volontairement simple et brève les caractéristiques principales de chaque série morphologique et typologique des différents services et du groupe divers. Les formes présentées en regard sont celles qui constituent les types ou variantes les plus représentatifs de chaque service ou de chaque série à l'intérieur d'un même service. Les références citées en appendice ne forment pas une liste exhaustive : seuls les parallèles proches ou identiques y sont mentionnés.

Les planches figurant en fin de texte décrivent toutes les variantes reconnues dans chaque groupe morphologique et typologique.

\section{LA CÉRAMIQUE SIGILLÉE}

(fig. 8 à 64 ; pl. 9 à 31 )

\section{DONNÉES NUMÉRIQUES DES DIX CONTEXTES SÉLECTIONNÉS (fig. 8 à 11)}

Cinq des dix contextes ont été dégagés en 1966 lors de la première campagne de fouilles : il s'agit de quatre dépotoirs ( Gobelet I " et " Gobelet II ", M.T.S., S3/S4) ainsi que du comblement d'une tranchée (T.N.E.). Les cinq autres correspondent à différents niveaux d'un grand dépotoir mis au jour en 1975 et fouillé en extension sur quatre zones contiguës : on a d'unc part lc remplissage de la couche ayant livré l'essentiel du matériel (U.S. 4 - zone 2, U.S. 3 - zone 4), d'autre part le comblement de niveaux immédiatement antérieurs (U.S. 1-2 et U.S. 5 - zone 4), (fig. 8).

Les histogrammes établis sur la base de cette première répartition (fig. 9) illustrent dans tous les cas la très nette prédominance du service I sur le service II, ainsi que le caractère anecdotique du groupe divers qui réunit les vases lisses ou décorés n'appartenant pas à l'un ou l'autre service. Le service I se maintient dans une fourchette comprise entre $70 \%(\mathrm{~S} 3 / \mathrm{S} 4)$ et $95 \%$ des vases (U.S. 4) ; le service II va de $4 \%$ (U.S. 4 ) à $26 \%$ (S3/S4) et le groupe divers ne dépasse 1 ou $2 \%$ que dans un des dix contextes ( $5 \%$ pour $\mathrm{S} 3 / \mathrm{S} 4$ ).

Ces premières données ont de quoi surprendre avant même d'aborder l'étude typo-chronologique proprement dite : le faible indice de fréquence des formes du service II, prépondérantes à Haltern (environ 7 avant J.-C. $/ 9$ après J.-C.) et dans les contextes augustéens classiques connus à ce jour, en particulier à Lyon, pose bien sûr des problèmes d'interprétation liés à la nature de dépôts d'atelier mais ne laisse pas d'évoquer également un horizon chronologique plus ancien que celui défini à Haltern : la juxtaposition des histogrammes des dix contextes de la Muette et de ceux effectués d'après les publications de Haltern (von Schnurbein, 1982) et de contextes lyonnais du Verbe-Incarné d'époque classique datés par référence à Haltern (Genin, 1993) mettent en lumière un très fort déséquilibre numérique dans les proportions service I/service II entre le matériel de l'atelier et celui de ces deux sites de consommation. La sigillée du Verbe-Incarné se calque presque parfaitement sur celle de Haltern, en dépit de sa faiblesse numérique relative (136 vases contre 1353 à Haltern) : le service II représente sur les deux sites plus de $50 \%$ des vases sigillés, le service I, 33 à $34 \%$ et le groupe divers, $15 \%$ (fig. 9).

\begin{tabular}{|l|r|r|r|r|r|r|r|r|r|r|r|}
\cline { 2 - 12 } \multicolumn{1}{c|}{} & G. I & G. II & U.S. 1 & U.S. 2 & U.S. 3 & U.S. 4 & U.S. 5 & T.N.E. & M.T.S. & S3/S4 & total \\
\hline S.I & 1863 & 2503 & 1580 & 864 & 676 & 2387 & 1513 & 6919 & 1261 & 321 & 19887 \\
\hline S.II & 155 & 270 & 248 & 75 & 74 & 110 & 230 & 1374 & 163 & 118 & 2817 \\
\hline divers & 22 & 22 & 18 & 10 & 9 & 17 & 31 & 277 & 22 & 25 & 453 \\
\hline total & $\mathbf{2 0 4 0}$ & 2795 & 1846 & $\mathbf{9 4 9}$ & $\mathbf{7 5 9}$ & $\mathbf{2 5 1 4}$ & $\mathbf{1 7 7 4}$ & $\mathbf{8 5 7 0}$ & $\mathbf{1 4 4 6}$ & $\mathbf{4 6 4}$ & $\mathbf{2 3 1 5 7}$ \\
\hline
\end{tabular}

Fig. 8. Première répartition des bords sigillés dans les trois grands groupes de vases. 

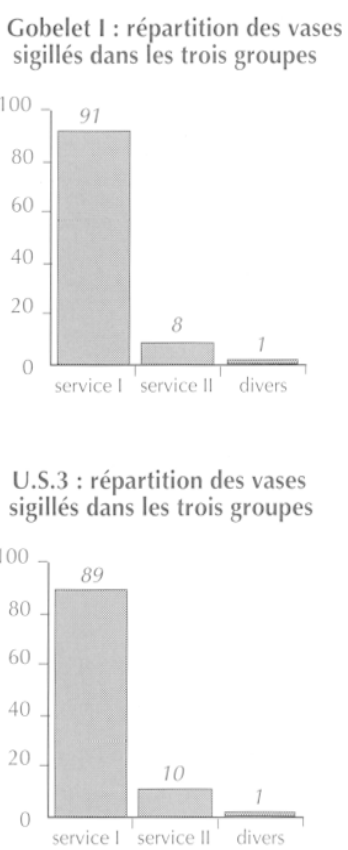

M.T.S : répartition des vases sigillés dans les trois groupes

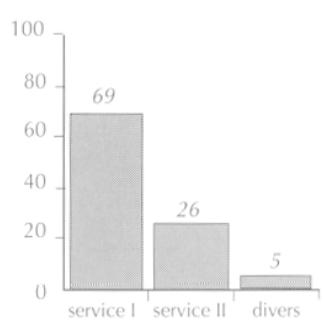

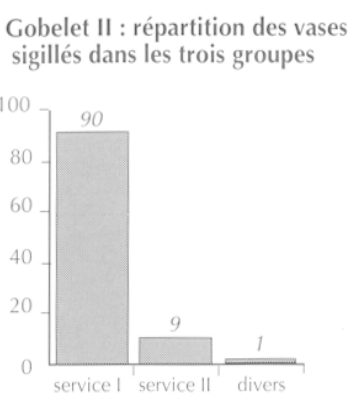

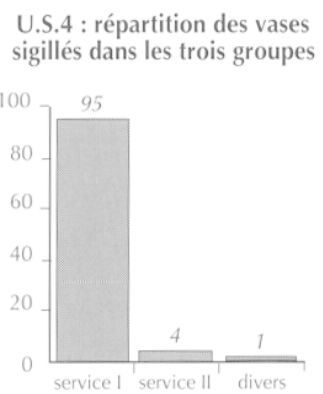

S3/S4 : répartition des vases sigillés dans les trois groupes

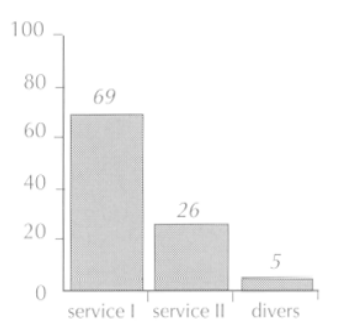

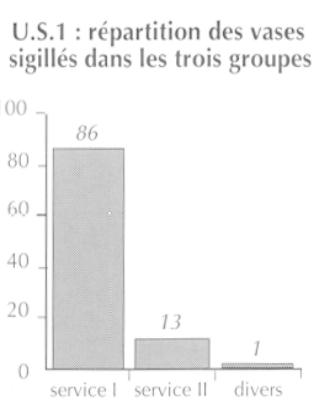

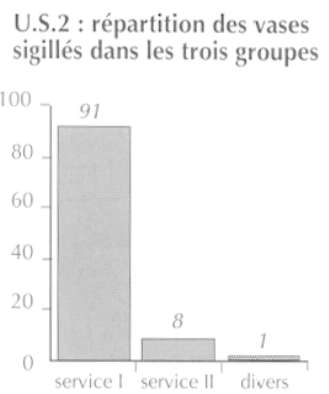

U.S.5 : répartition des vases sigillés dans les trois groupes

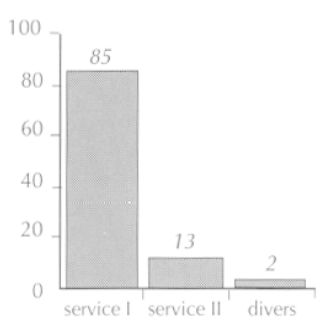

T.N.E : répartition des vases sigillés dans les trois groupes

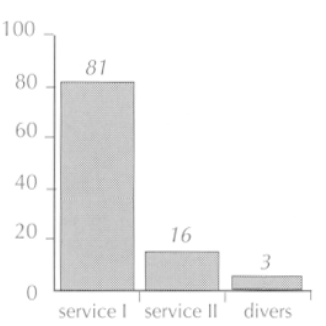

HALTERN : répartition des vases sigillés dans les trois groupes

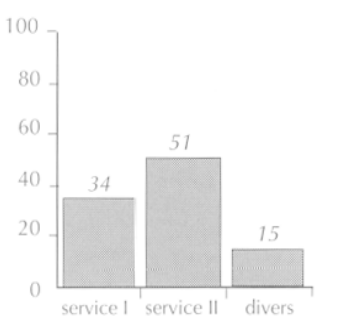

Verbe-Incarné : répartition des vases sigillés dans les trois groupes

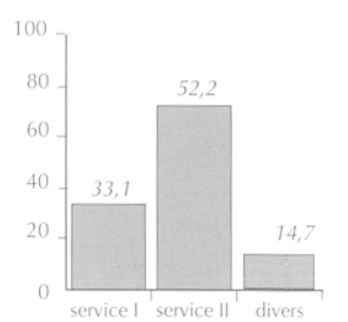

Fig. 9. Répartition par service des vases sigillés dans les ensembles de latelier et comparaison avec les domnées fournies par les sites de Haltern (d'après von Schnurbein, 1982) et du Verbe-Incamé (d'après Cienin, 1993).

De tels chiffres pourraient d'ailleurs suggérer d'cmblée qu'il existe une identité quasi parfaite entre les dix contextes de l'atelier, identité chronologique bien sûr et, partant, identité typologique.

D'une part, il a donc fallu dans un premicr temps examiner de façon très précise le mobilier issu des niveaux mis au jour en 1975 : l'étude a en fait révélé que ces niveaux appartiemnent au même horizon typo-chronologique stricto sensu. D'autre part, il est vite apparu que les ensembles appclés "Gobelet I " et "Gobelet II " ne formaient en fait qu'un seul et même dépotoir. L'analyse détaillée des formes sigillées de ces niveaux comme des trois autres contextes montre un grand nombre d'analogies d'un contexte à l'autre, mais fait également ressortir des dissemblances notables sur les plans typologique et numérique. Ces dissemblances bousculent l'impression d'homogénéité, voire d'uniformité, que donnent les histogrammes établis d'après la première répartition typologique des rases et nous ont amenés à distinguer deux aspects ou "faciès " dans les productions sigillées et à présenter le matériel à travers deux grands ensembles :

- ensemble I : "Gobelet I - Gobelet II »- U.S. 1 - U.S. 2 - U.S. 3 - U.S. 4 - U.S. 5 ;

- enscmble II : M.T.S. - T.N.E. - S3/S4.

Les tableaux qui suivent détaillent la répartition entre les différentes séries typologiques et morphologiques des services I-II et du groupe divers : ils constituent notre principale base de données pour l'étude et la présentation des productions sigillées (fig. 10 et 11 ). 


\section{ENSEMBLE I : ÉTUDE TYPOLOGIQUE ET STATISTIQUE}

LE SERVICE IB (fig. 12 et $13 ;$ pl. 9 à 12)

Les plats (pl. 9, 1-5)

Les deux variantes (53 ex.) n'appellent pas de commentaire particulier : elles se rattachent sans problème au type de plats IB connu sous Auguste (type 15 de Bolsena).

Les assiettes (fig. 12; pl. 9, 6-18 et pl. 10, 1-4)

Le groupe 1 (profils précoces) se distingue avant tout par son absence d'unité : c'est un chapelet de formes rarement semblables de l'une à l'autre et témoignant d'un faible degré de standardisation de la production. Ces profils sont totalement absents à Haltern et les rares parallèles mentionnés proviennent de Dangstetten et de Neuss. On note pourtant ici qu'il constitue plus de $92 \%$ des assiettes IB de l'ensemble I (pl. 9, 6-18).

Le groupe 2 est à l'inverse un groupe très homogène qui rassemble les assiettes que nous avons appelées « type Lyon " par assimilation aux bols IB ainsi dénommés par les auteurs allemands; des formes approchantes existent à Haltern mais les seuls parallèles exacts que l'on puisse citer sont attestés à Oberhausen. Il se réduit ici à $8 \%$ de l'effectif (pl. 10, 1-4).

Les bols (fig. 13; pl. 10, 5-15; pl. 11 et pl. 12)

Les groupes 1 à 3 rassemblent différents types de profils précoces inégalement attestés sur le limes. On peut assimiler les bols du groupe 1 au type Goudineau 16 (pl. 10, 5-15) et signaler quelques parallèles pour ceux du groupe 3 (pl. 11, 7-15), tandis que le groupe 2 (pl. 11, 16), en l'occurrence le plus homogène typologiquement, reste totalement inconnu sur les sites de référence. En termes numériques, ces trois groupes ajoutés ne représentent cependant que $14 \%$ des bols IB avec 636 exemplaires.

L'ensemble est en fait dominé par les groupes 4 (pl. 12, 1-7) et 5 (pl. 12, 8-12) qui comprennent un fort effectif de «bols type Lyon » et de formes satellites pour lesquels les références abondent sous Auguste : ces deux groupes classiques totalisent en effet $86 \%$ des bols IB.

L'image typologique qu'offre l'échantillon des bols du service IB est à forte dominante classique et contraste donc singulièrement avec celle qu'offraient les assiettes du même service.

\begin{tabular}{|l|c|c|}
\hline & ensemble I & ensemble II \\
\hline service IB & 5468 & 2923 \\
\hline service IB/IC & 2479 & 63 \\
\hline service IC & 3439 & 5515 \\
\hline service II & 1162 & 1655 \\
\hline autres & 129 & 324 \\
\hline total & 12677 & 10480 \\
\hline
\end{tabular}

Fig. 10. Répartition aypologique des vases sigillés dans les deux ensembles.

\begin{tabular}{|c|r|r|}
\hline & ensemble I & ensemble II \\
\hline service IB & 5468 & 2923 \\
\hline plats & 53 & 168 \\
assiettes & 913 & 887 \\
bols & 4502 & 1868 \\
\hline service IB/IC & 2479 & 63 \\
\hline plats & 32 & 0 \\
\hline assiettes & 1757 & 42 \\
\hline bols & 690 & 21 \\
\hline service IC & 3439 & 5515 \\
\hline plats & 83 & 607 \\
\hline assiettes & 2340 & 4508 \\
\hline bols & 1016 & 400 \\
\hline service II & 1162 & 1655 \\
\hline plats & 0 & 127 \\
\hline assiettes & 225 & 292 \\
\hline bols & 937 & 1236 \\
\hline divers lisses & 128 & 298 \\
\hline assiettes à bord oblique & 18 & 0 \\
\hline bols à bord oblique & 91 & 111 \\
\hline bols Ha. 10 & 1 & 103 \\
\hline bols Ha. 14 & 6 & 0 \\
\hline gobelets Ha. 16 & 2 & 73 \\
\hline formes & 2 & 1 \\
\hline & 2677 & 10480 \\
\hline
\end{tabular}

Fig. 11. Répartition typologique et morphologique des vases sigillés dans les deux ensembles. 
LE SERVICE IB/IC (fig. 14 et pl. 13)

Ce service réunit, comme son nom l'indique, des plats (pl. 13, 1-3), des assiettes (pl. 13, 4-6) et des bols (pl. 13, 79) qui apparaisscnt comme des formes intermédiaires entre les services IB et IC tels qu'ils sont traditionnellement définis; il montre un aspect homogène même si on relève, en particulier dans le groupe des plats, des variations sensibles dans les profils de lèvres.

Ces formes s'avèrent extrêmement rares sur le limes comme ailleurs : les seuls parallèles que l'on puisse signaler sont des plats mais surtout des assiettes classés à Neuss en « service I type Lyon » et apparemment peu nombreux au sein du service I. Les données numériques de l'atelier montrent pourtant qu'il ne s'agit pas d'un groupe marginal dans la production par rapport aux services IB et IC : les trois séries (plats, assiettes, bols) comprennent en effet 2479 vases soit $22 \%$ du total des vases du service I de l'ensemble I.

LE SERVICE IC (fig. 15 à 17; pl. 14 à 19)

Les plats (fig. 15 et pl. 14)

Au groupe 1 correspondent des plats qui, bien qu'appartenant sans ambiguité au service IC, restent très rares sur les sites de référence : un seul parallèle à Haltern et quelques profils similaires à Neuss dans le groupe « service I type Lyon » cité plus haut (pl. 14, 1-6).

Le groupe 2, qui est ici nettement majoritaire, présente un aspect très homogène et se rattache parfaitement aux profils classiques de plats IC connus sur le limes comme en Italie ou en Gaule : il correspond aux variantes b et c du type Goudineau 17 (pl. 14, 7-15).

Les assiettes (fig. 16; pl. 15 à 17)

Le groupe 1 (pl. 15) apparaît comme un agglomérat de formes non normalisées dont la variété interdit tout classement cohérent, même si certains profils montrent déjà des traits morphologiques propres au service IC, et notamment la division tripartite de la paroi interne. Il est intéressant de souligner que ce groupe quasiment absent sur le limes, rassemble pourtant $42 \%$ des assiettes IC avec 986 exemplaires.

À l'inverse, les groupes 2 (pl. 16, 1-17) et 3 (pl. 16, 1823) constituent bel et bien des types homogènes, paradoxalement absents des sites de référence, et formant ici

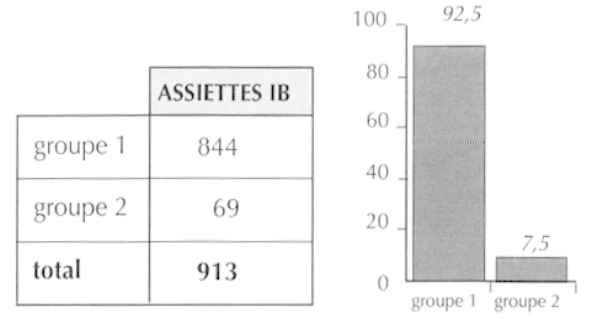

Fig. 12. Ensemble I, représentation respective des deux groupes d'assiettes du service IB.

\begin{tabular}{|l|c|}
\cline { 2 - 2 } \multicolumn{1}{c|}{} & BOLS IB \\
\hline groupe 1 & 341 \\
\hline groupe 2 & 85 \\
\hline groupe 3 & 210 \\
\hline groupe 4 & 2274 \\
\hline groupe 5 & 1592 \\
\hline total & 4502 \\
\hline
\end{tabular}

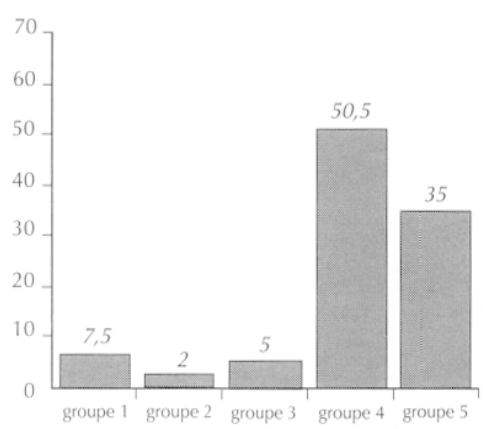

Fig. 13. Ensemble I, représentation respective des cinq groupes de bols du service $I B$.

\begin{tabular}{|l|c|}
\cline { 2 - 2 } \multicolumn{1}{c|}{} & SERVICE IB/IC \\
\hline plats & 32 \\
\hline assiettes & 1757 \\
\hline bols & 690 \\
\hline total & $\mathbf{2 4 7 9}$ \\
\hline
\end{tabular}

Fig. 14. Ensemble I, représentation respective des trois groupes de vases du service $I B / I C$.

\begin{tabular}{|l|c|}
\cline { 2 - 2 } \multicolumn{1}{c|}{} & PLATS IC \\
\hline groupe 1 & 11 \\
\hline groupe 2 & 72 \\
\hline total & 83 \\
\hline
\end{tabular}

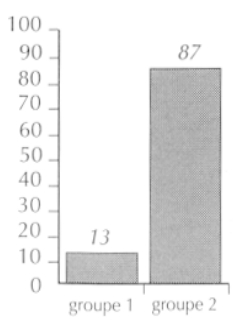

Fig. 15. Ensemble I, représentation respective des deux groupes de plats du service IC. 
$11 \%$ et $35 \%$ de l'effectif. On remarque une nette évolution vers les formes classiques avec les assiettes du groupe 4 qui évoquent de façon plus évidente le type Goudineau 23 , en dépit de variations encore fréquentes dans les profils de lèvres et la division interne des parois qui n'obëit pas toujours au schéma bipartite propre à ce type classique. Ce groupe attesté à Haltern comme à Neuss se réduit à $2 \%$ des assiettes IC (pl. 17, 1-7).

Les assiettes du groupe 5 qui offrent enfin des profils classiques pour lesquels on pourrait énumérer un grand nombre de parallèles sur tous les sites augustéens ne représentent encore que $10 \%$ des assiettes IC de l'ensemble I (pl. 17, 8-19).

Les bols (fig. 17; pl. 18 et 19)

Les groupes 1 (pl. 18, 1-8) et 2 (pl. 18, 9-16) montrent deux types précoces figurant en quelques exemplaires à Haltern ou à Dangstetten et qui correspondent ici à 8,5 \% et $16 \%$ des bols du service IC.

Les bols du groupe 3 (pl. 19, 1-4) forment un type homogène de facture classique qui, bien que faible sur le plan numérique (2\%), est parfaitement connu sur les sites de référence.

Au groupe 4 (pl. 19, 5-10) se rattachent les profils les plus classiques de bols IC extrêmement abondants à Haltern et à Neuss comme ailleurs, et qui prédominent ici de façon évidente avec plus de $73 \%$ de l'effectif. Ce très fort pourcentage des groupes classiques au sein des bols IC contraste avec l'aspect qu'offraient les assiettes du même service où les formes standardisées du groupe 5 ne comptaient que $10 \%$ des vases.

LE SERVICE II (fig. 18 et 19 ; pl. 20 à 22)

Les assiettes (fig. 18; pl. 20, 1-7)

Les assiettes du service II appartiennent dans leur quasi-totalité à un même type caractérisé par des bords incurvés et non profilés. Ce type qui n'existe pas à Haltern, et dont les contextes augustéens lyonnais, qu'ils soient d'époque précoce ou classique, n'ont pas livré un seul exemplaire, apparaît à Neuss et à Dangstetten en proportions infimes par rapport aux formes classiques du service II (pl. 20, 1-5).

Les deux assiettes qui font exception dans ce lot possèdent des bords profilés à division interne bipartite qui se

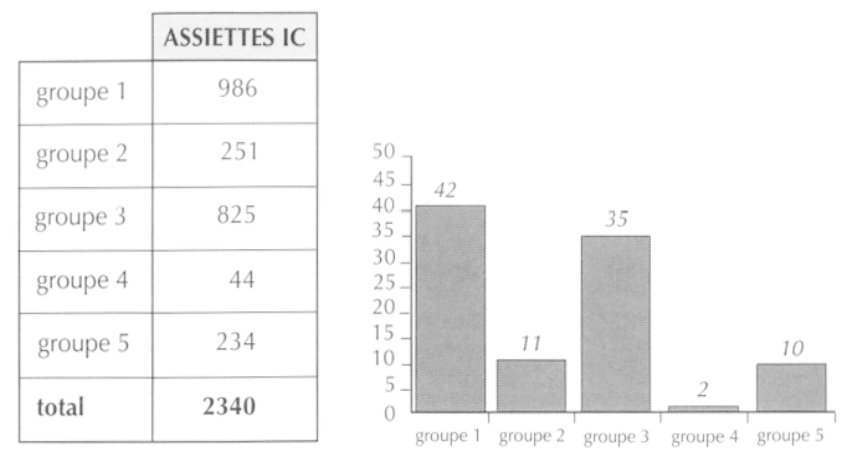

Fig. 16. Ensemble I, représentation respective des cing groupes d'assiettes du service IC.

\begin{tabular}{|l|c|}
\cline { 2 - 2 } \multicolumn{1}{c|}{} & BOLS IC \\
\hline groupe 1 & 88 \\
\hline groupe 2 & 162 \\
\hline groupe 3 & 19 \\
\hline groupe 4 & 747 \\
\hline total & 1016 \\
\hline
\end{tabular}

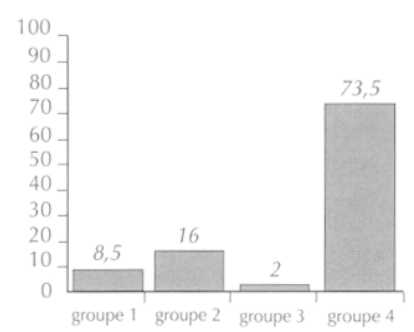

Fig. 17. Ensemble I, représentation respective des quatre groupes de bols du service IC.

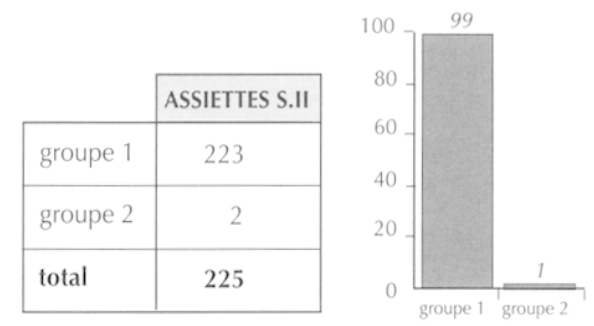

Fig. 18. Ensemble I, représentation respective des deux groupes d'assiettes du service II.

\begin{tabular}{|l|c|}
\cline { 2 - 2 } \multicolumn{1}{c|}{} & BOLS S.II \\
\hline groupe 1 & 571 \\
\hline groupe 2 & 47 \\
\hline groupe 3 & 113 \\
\hline groupe 4 & 194 \\
\hline groupe 5 & 12 \\
\hline total & 937 \\
\hline
\end{tabular}

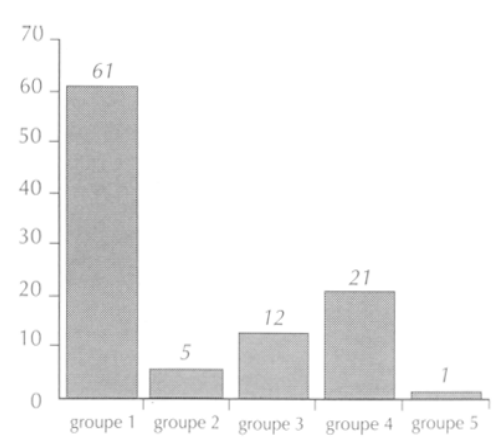

Fig. 19. Ensemble I, représentation respective des cinq groupes de bols du service II. 
rapprochent davantage des assiettes classiques du service II (pl. 20, 6-7).

Les bols (fig. 19; pl. 20, 8-18; pl. 21 et 22)

Neuf cent trente-sept bols déterminent cinq groupes très homogènes typologiquement.

Le groupe 1 (pl. 20,8-18) rassemble des bols à bord court, très évasé, dont la morphologie d'ensemble définit une variante sans doute précoce des bols du service II : ce groupe est extrêmement rare sur le limes, si l'on excepte quelques exemplaires d'Oberaden, de Mayence et de Bonn recensés par $S$. von Schnurbein, mais il reste inédit à Lyon, sur les sites de consommation. On retiendra qu'il constitue $61 \%$ des bols du service II de l'ensemble I.

Au groupe 2 (pl. 21, 1-3) correspond un type complètement différent : il s'agit de bols aux petites dimensions montrant des bords droits délimités par des bourrelets guillochés parfaitement réguliers : ce type est connu sous Auguste, que ce soit sur le limes ou dans les contextes lyonnais, et représente ici う̌ \% seulement de l'effectif.

Les bols classés dans le groupe 3 (pl. 21, 4-13) offrent en revanche des profils tout à fait originaux et, de fait, totalement inconnus dans les typologies existantes : les lèvres sont hautes, à face externe arrondie ou oblique et dégagées à l'intéricur par une gorge large et très accentuée. Ce type inédit compte $12 \%$ des bols du service II.

Les bols du groupe 4 (pl. 22, 1-8), qui se rapprochent davantage des formes classiques, sont un peu mieux représentés sur le limes, mais toujours, semble-t-il, en proportions modestes. Il arrive ici en seconde position derrière le groupe 1 avec $21 \%$ des vases.

I.es formes classiques de bols du service II (type Haltern 8/Goudineau 27) se retrouvent en quelques exemplaires isolés dans le groupe 5 qui réalise à peine $1 \%$ de l'effectif (pl. 22, 9-11).

DIVERS (fig. 20 et pl. 23)

Le groupe divers comprend cent vingt-neuf vases qui se répartissent entre huit séries distinctes (fig. 20).

Les assiettes et les bols à bord oblique, hérités d'anciens types de la céramique campanienne puis repris au tout début de la production par les potiers arétins, forment l'essentiel de cet échantillon. Contrairement aux assiettes, les bols ont connu un succès plus durable, comme le montrait déjà le mobilier de Haltern où ils sont classés dans le type 10A. Les exemplaires de l'atelier sont typologiquement semblables à leurs homologues de Haltern et possèdent tous de très grands diamètres avoisinant $200 \mathrm{~mm}$. Ils offrent néanmoins, pour quelques-uns d'entre eux, des surfaces d'aspects variés : vernis orange à rouge sombre, grésés et parfaitement uniformes ou vernis peu adhérents de teintes brunes à noires équivalents aux engobes connus sur les bols à bord oblique produits en imitations sous Auguste. Les bols Haltern 10A constituent plus de $70 \%$ des vases du groupe divers (pl. 23, 1-3).

Dix-neuf autres vases lisses déterminent des types qui, bien que répertoriés à Haltern, sont extrêmement rares de manière générale et au premier rang desquels figurent les couvercles Haltern 17 dont on compte seulement cinq exemplaires dans le matériel du camp (pl. 23, 10); les gobelets Haltern 16 (pl. 23, 4), les bols Haltern 10 (pl. 23, 5) et les tasses Haltern 14 (pl. 23, 6-7) font eux aussi partie de ces formes lisses toujours marginales sous Auguste et isolées au sein de la masse du service I et du service II.

\begin{tabular}{|l|c|}
\hline \multicolumn{1}{|c|}{ TYPES } & VASES \\
\hline assiettes à bord oblique & 18 \\
\hline bols à bord oblique & 91 \\
\hline bols Ha.10 & 1 \\
\hline bols Ha.14 & 6 \\
\hline gobelets Ha.16 & 2 \\
\hline couvercles Ha.17 & 8 \\
\hline formes rares & 2 \\
\hline décorée & 1 \\
\hline total & 129 \\
\hline
\end{tabular}

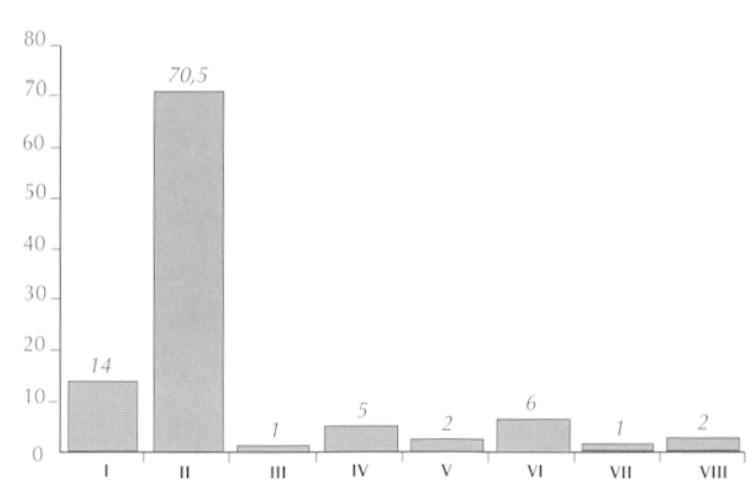

Fig. 20. Ensemble I, représentation respective des huit séries du groupe divers. 
Une dernière forme lisse est matérialisée par deux coupes ou gobelets à bord court, eversé et légèrement concave qui n’existent pas dans la typologie de Haltern mais qui éroquent quelques gobelets de Neuss (pl. 23, 8-9). Il convient enfin de signaler pour information la présence d'un fragment moulé montrant un départ d'oves et un décor végétal (non illustré).

\section{ENSEMBLE II : ÉTUDE TYPOLOGIQUE ET STATISTIQUE}

LE SERVICE IB (fig. 21 et $22 ;$ pl. 24)

Les plats (pl. 24, 1-6)

I.es deux variantes de plats IB recomnues dans l'ensemble I restent inchangées.

Les assiettes (fig. 21 et pl. 24, 7-9)

Le groupe 2 des " assiettes type Iyon " qui constituait $8 \%$ de l'effectif culmine à $95 \%$ dans l'ensemble II (pl. 24 , 7-9). Ce processus de normalisation a pour corollaire une chute brutale des profils précoces du groupe 1.

LES BOLS (fig. $22 ;$ pl. 24, 10-16)

Comme le montrent clairement ce tableau et l'histogramme qui l’accompagne, les groupes précoces 1 à 3 sont réduits à néant et l'essentiel des bols du service IB de l'ensemble II est désormais formé par les bols " type Iyon " et assimilés : groupe 4 (pl. 24, 10-15) et groupe s (pl. 24, 16).

\section{LE SERVICE IB/IC (fig. 23)}

Ce service à la fois original et bien homogène typologiquement ne comprend plus que 63 vases, soit 42 assiettes et 21 bols qui constituent à peine $1 \%$ du service I dans l'ensemble II contre $22 \%$ dans l'ensemble I (non illustrés).

LE SERVICE IC (fig. 24 à 26 ; pl. 25 et 26 )

Les plats (fig. $24 ;$ pl. 25, 1-8)

Le groupe 1 déjà faiblement représenté dans l'ensemble I, a presque complètement disparu au profit du

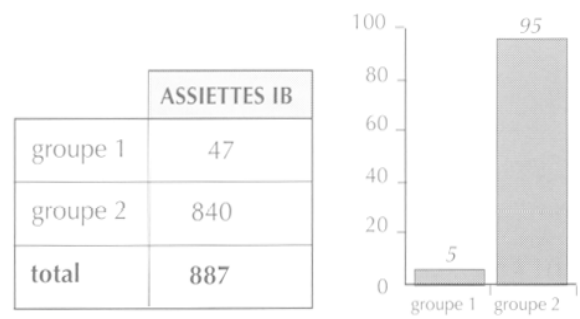

Fig. 21. Einsemble II, représentation respective des deux groupes d'assiettes du service IB.
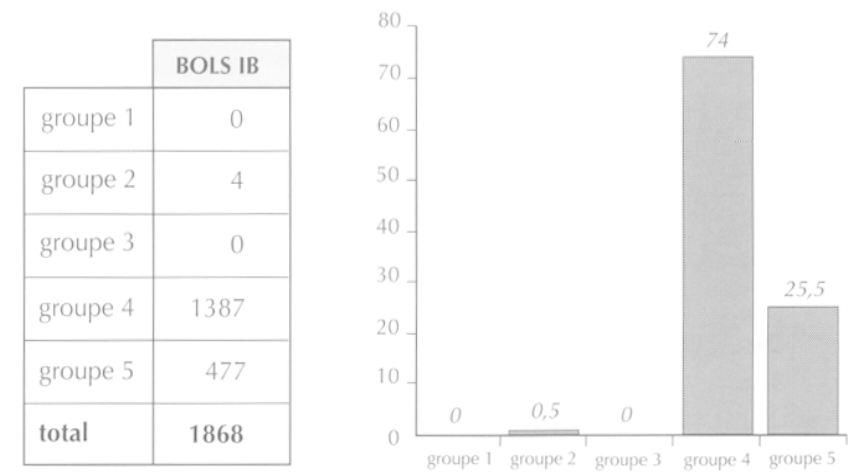

Fig. 22. Ensemble II, représentation respective des cinq groupes de bols du service IB.

\begin{tabular}{|l|c|}
\cline { 2 - 2 } \multicolumn{1}{c|}{} & SERVICE IB/IC \\
\hline plats & 0 \\
\hline assiettes & 42 \\
\hline bols & 21 \\
\hline total & 63 \\
\hline
\end{tabular}

Fig. 23. Ensemble II, représentation respective des deux groupes de vases du service $I B / I C$ :

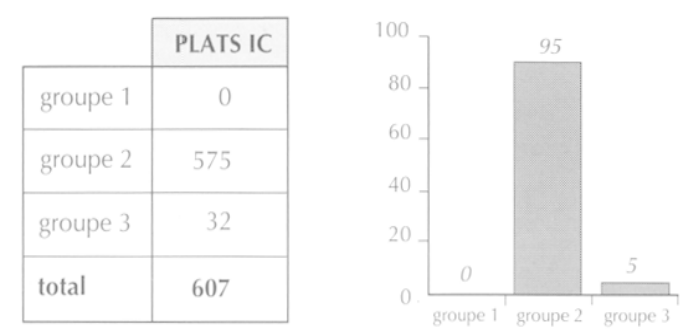

Fig. 24. Ensemble II, représentation respective des trois groupes de plats du senvice IC: 
groupe 2 (formes classiques) qui culmine à $95 \%$ des plats du service IC. On note qu'une trentaine de bords, de morphologie comparable à celle des assiettes IC classiques, composent un nouveau groupe 3 (pl. 25, 1). Un plat possède un bord oblique se raccordant au fond par une partie bombée à l'intérieur et droite à l'extérieur : cette particularité se rencontre sur les plats de type Haltern 3/Goudineau 28 (pl. 25, 8).

Les assiettes (fig. 25; pl. 25, 9-18)

Si l'on excepte quatorze assiettes du groupe 1 (profils précoces non standardisés), on voit que l'ensemble est définitivement dominé par les formes classiques du groupe 5 qui se caractérisent par une très forte homogénéité typologique et correspondent parfaitement au faciès des assiettes du service IC de l'horizon Haltern (pl. 25, 9-18).

Les bols (fig. 26 et pl. 26)

Si les quatre groupes initialement définis sont attestés de même dans l'ensemble II, leur répartition numérique montre également de nets changements : les profils faiblement normalisés des groupes 1 et 2 se réduisent à quelques exemplaires (pl. 26, 1), cédant le pas aux deux types classiques des groupes 3 (pl. 26, 2-4) et 4 (pl. 26, 510). D'une part, le groupe 3 passe de $2 \%$ à $13 \%$ des bols IC, d'autre part le groupe 4 confirme sa prééminence en augmentant encore de $73 \%$ à $84 \%$ de l'effectif (fig. 26).

LE SERVICE II (fig. 27 et 28 ; pl. 27 à 29)

Les plats (pl. 27, 1-11)

Ce groupe morphologique absent de l'ensemble I comprend 127 exemplaires dont 126 de type Haltern 2 et un seul de type Haltern 3 .

Les plats Haltern 2 présentent des variantes qui ne justifient pas cependant un classement par groupes : les bords, qu'ils soient incurvés ou droits possèdent une triple division externe et interne bien marquée et seuls quelques exemplaires isolés offrent des bords lisses non divisés ou à division interne bipartite (pl. 27, 1-5).

Le plat Haltern 3 ne diffère des précédents que par un double ressaut à la liaison bord/fond; la fragmentation du matériel empêche de savoir s'il s'agit ou non du seul plat de type 3 au sein de cet échantillon (pl. 27, 11).

\begin{tabular}{|l|c|}
\cline { 2 - 2 } \multicolumn{1}{c|}{} & ASSIETTES IC \\
\hline groupe 1 & 14 \\
\hline groupe 2 & 0 \\
\hline groupe 3 & 0 \\
\hline groupe 4 & 0 \\
\hline groupe 5 & 4494 \\
\hline total & 4508 \\
\hline
\end{tabular}

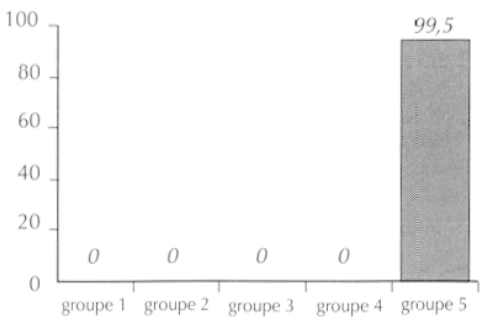

Fig. 25. Ensemble II, représentation respective des cinq groupes d'assiettes du service IC.

\begin{tabular}{|l|c|}
\cline { 2 - 2 } \multicolumn{1}{c|}{} & BOLS IC \\
\hline groupe 1 & 1 \\
\hline groupe 2 & 10 \\
\hline groupe 3 & 53 \\
\hline groupe 4 & 336 \\
\hline total & $\mathbf{4 0 0}$ \\
\hline
\end{tabular}

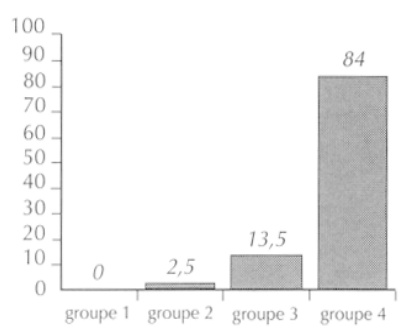

Fig. 26. Ensemble II, représentation respective des quatre groupes de bols du service IC.

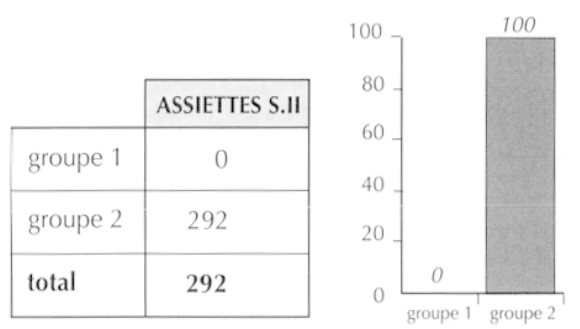

Fig. 27. Ensemble II, représentation respective des deux groupes d'assietles du service II.

Les assiettes (fig. 27; pl. 27, 12-20)

Le groupe 1 (bords incurvés, non profilés) qui était presque exclusivement représenté dans l'ensemble I a donc complètement disparu au profit du groupe 2 qui rassemble un lot conséquent d'assiettes montrant différentes variantes classiques du type Haltern 2 (pl. 27, 12$20)$. 
Les bols (fig. 28 ; pl. 28 et 29 )

Le groupe 1 (bords courts peu profilés et très évasés) qui formait $61 \%$ des bols de l'ensemble I et le groupe 3 (type inédit, 12\%), ne sont plus attestés dans l'ensemble II. En fait, seules restent les formes pour lesquelles on pouvait établir des parallèles sur les sites de référence : le groupe 2 (petits bols à bords droits) constituait $5 \%$ du lot et passe ici à $29 \%$ (pl. 28, 1-6); le groupe 4, proche des formes classiques, augmente également jusqu'à représenter la moitié des bols du service II (pl. 28, 7-14). Le groupe 5 (pl. 29), qui rassemble les variantes classiques les plus fréquentes sous Auguste et dont l'effectif était quasiment nul (1\%), livre $21 \%$ des bols de l'ensemble 2 (fig. 28).

Drvers (fig. 29 ; pl. 30 et 31 )

Trois des huit séries de vases lisses reconnues dans l'ensemble I n'existent plus dans l'ensemble II : assiettes à bord oblique, bols Haltern 14, couvercles Haltern 17.

Les bols à bord oblique accusent une baisse sensible avec $34 \%$ contre $70 \%$ précédemment (pl. 30, 1-4); en revanche, les gobelets Haltern 16 (pl. 30,5-10) et les bols Haltern 10 (pl. 31, 1-4), qui étaient auparavant très faiblement représentés, constituent ici $23 \%$ et $32 \%$ des rases du groupe divers. Parmi onze vases de type indéterminé (pl. 31, 5-8), trois possèdent une lèrre arrondie à décor guilloché et une paroi légèrement oblique évoquant quelques profils de Neuss (pl. 31, 5).

La sigillée décorée est illustrée par quatre vases distincts : des calices Drag. 11 (pl. 31, 9-10), un gobelet Drag. 13 (pl. 31, l1), ainsi qu'une coupe représentée par un fond de grand diamètre à pied fin et mouluré (pl. 31, 12).

\section{LA CHRONOLOGIE DU MOBILIER}

Les deux grands critères habituellement retenus sur un site de consommation afin de dater la sigillée augustéenne sont d'une part les fréquences respectives des services I et II, d'autre part la répartition des services IB et IC à l'intérieur du service I.

La première répartition service $\mathrm{I} /$ service II effectuée pour les deux ensembles donne deux images similaires dont le seul mérite est de prouver un fort décalage avec le matériel du limes, en particulier avec la sigillée de Haltern (cf. supra, fig. 9). En revanche, la seconde répartition, qui prend en compte les séries IB et IC auxquelles s'ajoute ici

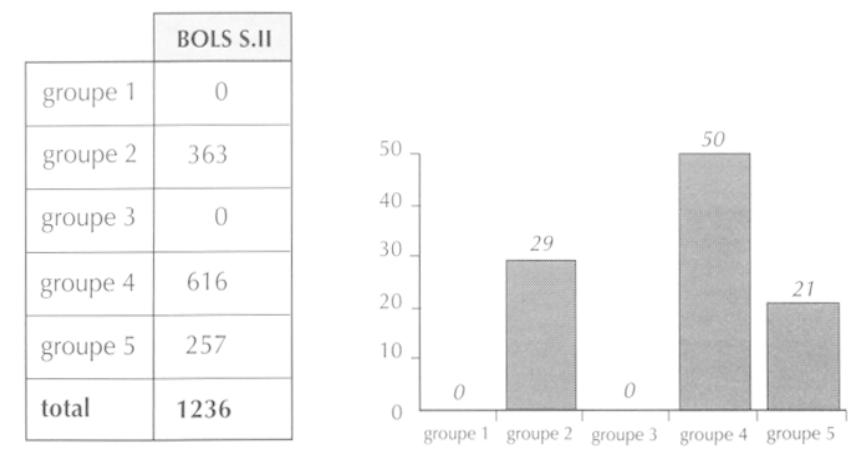

Fig. 28. Ensemble II, représentation respective des cinq groupes de bols du service II.

\begin{tabular}{|l|c|}
\hline \multicolumn{1}{|c|}{ TYPES } & VASES \\
\hline assiettes à bord oblique & 0 \\
\hline bols à bord oblique & 111 \\
\hline bols Ha.10 & 103 \\
\hline bols Ha.14 & 0 \\
\hline gobelets Ha.16 & 73 \\
\hline couvercles Ha.17 & 0 \\
\hline formes rares & 11 \\
\hline décorée & 26 \\
\hline total & 324 \\
\hline
\end{tabular}

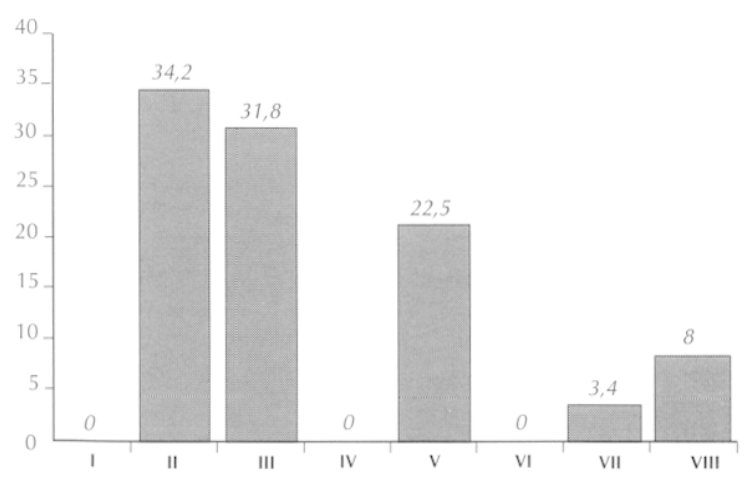

Fig. 29. Ensemble II, représentation respective des huit séries du groupe divers.

la série IB/IC, permet de reconnaitre deux faciès différents : dans l'ensemble II, baisse sensible du service IB, quasi-disparition du service IB/IC, nette augmentation du service IC (cf. supra, fig. 10 et 11). 
L'analyse fine des sigillées de chaque ensemble vient confirmer la valeur d'indice chronologique de ces déséquilibres numériques.

Dans l'ensemble I, le service IB se caractérise par la nette prćdominance de formes peu normalisées, en particulier dans le groupe des assiettes. La remarque vaut également pour les assiettes du service IC parmi lesquelles les types classiques que l'on retrouve dans l'horizon Haltern constituent à peine $10 \%$ de l'effectif. Cette opposition entre formes non standardisées et formes classiques est moins accentuée dans les groupes de bols IB et IC où on note parallèlement une supériorité incontestable des principaux types attestés sur le limes, et en premier lieu à Haltern. Par ailleurs, la présence et l'importance relative du service IB/IC sont autant d'autres éléments caractéristiqucs de l'ensemble I : ce service homogène, qui rassemble plus de $20 \%$ des vases sigillés, s'avère curieusement très rare sur les sites de référence.

Le service II présente une image originale, tant en ce qui concerne les assiettes que les bols. Les assiettes appartiennent dans leur quasi-totalité à un seul type complètement absent à Haltern et pour lequel on ne retrouve que quelques parallèles isolés à Dangstetten ou à Neuss. Les bols se singularisent par la prééminence de formes soit inédites (groupe 3), soit rarissimes sur les sites de référence (groupe 1) et qui constituent $73 \%$ de l'échantillon.

La sigillée de l'ensemble II montre des changements radicaux dans la composition et la répartition des groupes, séries et types définis d'après le mobilier de l'ensemble I.

Les groupes précoces d'assiettes IB et IC cèdent le pas aux groupes classiques qui représentent désormais l'essentiel des vases dans chacun des deux services. Parmi les bols IB et IC, les formes standardisées déjà majoritaires dans l'ensemble I (bols IB groupes 4 et 5 , bols IC groupes 3 et 4 ) assoient définitivement leur domination. Le service $\mathrm{IB} / \mathrm{IC}$ passe, quant à lui, de $20 \%$ à $0,5 \%$ des vases sigillés.

Le service II met à son tour en évidence une très nette évolution typologique : les assiettes précoces (groupe 1) ne sont plus attestées et l'on trouve enfin une série de profils réunissant les principaux traits morphologiques du type Haltern 2 (groupe 2). La composition typologique des bols subit, elle aussi, des modifications évidentes : les groupes 1 et 3 ont complètement disparu tandis que les trois autres groupes voient leur représentation globale augmenter de façon très nette; encore faut-il nuancer pour le groupe des formes classiques (groupe 5 ) qui reste loin derrière les groupes 2 et 4 . Les céramiques sigillées des deux ensembles définissent donc deux images très différentes qui correspondent, selon toute vraisemblance, à deux périodes de production. Les points de rupture observés peuvent se résumer dans le tableau VI.

Tabl. VI. Les deux périodes de production des céramiques sigillées de l'atelier de la Muette.

\begin{tabular}{|l|l|}
\hline ENSEMBLE I & ENSEMBLE II \\
\hline $\begin{array}{l}\text { abondance des assiettes précoces } \\
\text { du service IB }\end{array}$ & $\begin{array}{l}\text { absence quasi totale des assiettes précoces } \\
\text { du service IB }\end{array}$ \\
\hline $\begin{array}{l}\text { abondance relative des formes } \\
\text { du service IB/IC }\end{array}$ & $\begin{array}{l}\text { absence quasi totale des formes } \\
\text { du service IB/IC }\end{array}$ \\
\hline $\begin{array}{l}\text { abondance des assiettes précoces } \\
\text { du service IC }\end{array}$ & $\begin{array}{l}\text { absence quasi totale des formes précoces } \\
\text { du service IC }\end{array}$ \\
\hline $\begin{array}{l}\text { faiblesse numérique des assiettes IC } \\
\text { horizon Haltern }\end{array}$ & $\begin{array}{l}\text { présence quasi exclusive des assiettes IC } \\
\text { horizon Haltern }\end{array}$ \\
\hline $\begin{array}{l}\text { présence quasi exclusive d'un type } \\
\text { précoce dans les assiettes du service II }\end{array}$ & $\begin{array}{l}\text { absence totale de ce type précoce et présence } \\
\text { exclusive de profils classiques Haltern 2 }\end{array}$ \\
\hline $\begin{array}{l}\text { très forte représentation de variantes rares } \\
\text { ou inédites dans les bols du service II }\end{array}$ & $\begin{array}{l}\text { disparition totale des variantes précoces et nette } \\
\text { augmentation des profils classiques de type Haltern } 8\end{array}$ \\
\hline
\end{tabular}

L'ensemble I s'inscrit dans un horizon chronologique précoce, sans doute légèrement antérieur à celui de Dangstetten. L'ensemble II évoque davantage un horizon classique type Haltern, sans qu'il y ait, pour cela, adéquation parfaite, loin s'en faut, sur tous les points abordés. L'état actuel de nos connaissances sur les horizons chronologiques du limes incite cependant à la prudence : en effet, bien que publié de façon détaillée, le mobilier de Dangstetten, par exemple, n'a pas encore fait l'objet d'une publication de synthèse fondée sur une étude statistique permettant d'établir différentes sortes de comparaisons. Par ailleurs, mais là surgissent d'autres questions que nous aborderons ultérieurement, on peut suggérer, au vu de l'échantillon des marques de potiers attestées sur ce site, qu'il n'y avait pas de relations commerciales privilégiées entre l'atelier de la Muette et ce camp militaire. Parallèlement, les comparaisons avec Neuss, site dont l'occupation couvre une plus longue période et dont le matériel n'a pu être soumis à des analyses physico-chimiques en raison de son altération, s'avèrent plus difficilement exploitables. Il n'en est pas de même à Haltern, camp pour lequel on dispose d'une étude directement utilisable et où les analyses physico-chimiques de pâtes 
ont prouvé la part importante des productions lyonnaises dans l'approvisionnement en sigillée.

La mise en parallèle des histogrammes de fréquence des différents services incluant la séparation entre service IB et IC, établis d'après les ensembles I et II d'une part et d'après la sigillée de Haltern d'autre part, illustre de forts décalages et confère des particularités inattendues aux productions lyonnaises (fig. 30 à 32). Si on compare en termes de présence/absence les trente-deux groupes définis à la Muette et le matériel du camp, on s'aperçoit que huit groupes seulement y sont bien représentés : bols IB4, plats IC-2, assiettes IC-5, bols IC-4, plats II, assiettes II-2, bols II-2, bols II-5 (fig. 33). Les comparaisons entre les sigillées de l'ensemble I et celles de Haltern s'avèrent la plupart du temps totalement infrucueuses, quel que soit l'angle de vue adopté : force est de constater que l'atelier a fabriqué, bien avant l'installation du camp, des milliers de vases dont la destination finale nous échappe totale-

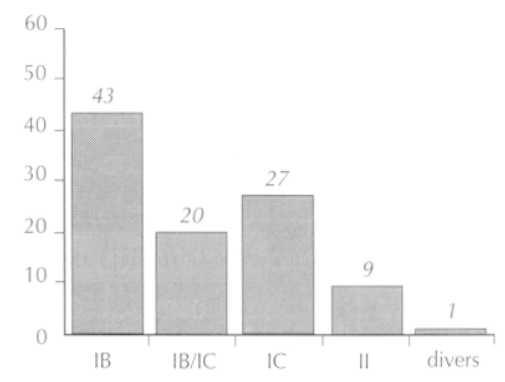

Fig. 30. Ensemble I, pourcentages respectifs des cinq groupes de vases sigillés. ment ou presque. On pouvait aussi s'attendre à unc meilleure adéquation entre les sigillées de l'ensemble II et celles trouvées sur le camp, que ce soit dans la composition globale ou dans la répartition typologique et numérique des vases : il n'y a pas identité à proprement parler entre ce que nous connaissons aujourd'hui des productions de la seconde période et ce qui a été utilisé sur un

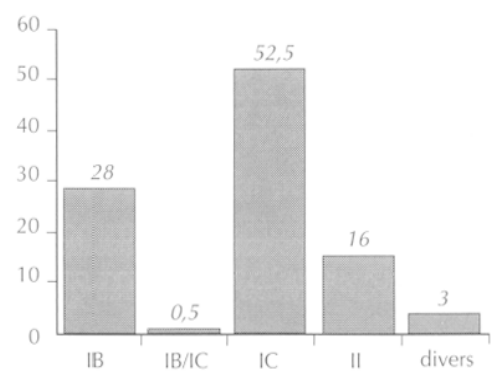

Fig. 31. Ensemble II, pourcentages respectifs des cinq groupes de vases sigillés.

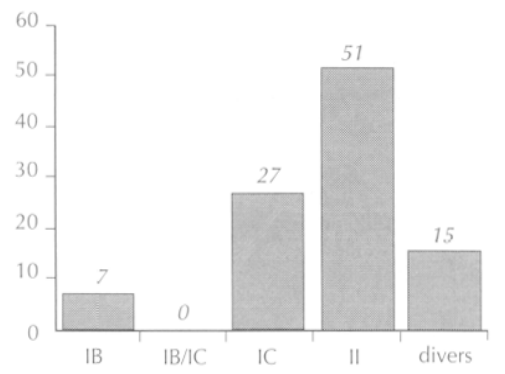

Fig. 32. Haltern, pourcentages respectifs des cinq groupes de vases sigillés.

\begin{tabular}{|c|c|c|c|}
\hline MUETTE & HALTERN & MUETTE & HALTERN \\
\hline plats IB gr1 & peu abondants & plats IC gr1 & absents \\
\hline plats IB gr2 & peu abondants & plats IC gr2 & très abondants \\
\hline assiettes IB gr1 & peu abondantes & assiettes IC gr1 & absentes \\
\hline assiettes IB gr2 & absentes & assiettes IC gr2 & absentes \\
\hline bols IB gr1 & absents & assiettes IC gr3 & absentes \\
\hline bols IB gr2 & absents & assiettes IC gr4 & peu abondantes \\
\hline bols IB gr3 & absents & assiettes IC gr5 & très abondantes \\
\hline bols IB gr4 & très abondants & bols IC gr1 & peu abondants \\
\hline bols IB gr5 & abondants & bols IC gr2 & absents \\
\hline plats IB/IC & absents & bols IC gr3 & abondants \\
\hline assiettes IB/IC & absentes & bols IC gr4 & très abondants \\
\hline bols IB/IC & absents & & \\
\hline
\end{tabular}

\begin{tabular}{|l|l|}
\hline \multicolumn{1}{|c|}{ MUETTE } & \multicolumn{1}{c|}{ HALTERN } \\
\hline plats II & très abondants \\
\hline assiettes II gr1 & absentes \\
\hline assiettes II gr2 & très abondantes \\
\hline bols II gr1 & absents \\
\hline bols II gr2 & très abondants \\
\hline bols II gr3 & absents \\
\hline bols II gr4 & abondants \\
\hline bols II gr5 & très abondants \\
\hline & \\
& \\
\end{tabular}

Fig. 33. Présences/absences à Hallem des différents groupes typologiques de la . Muette. 


\begin{tabular}{|l|c|c|}
\hline SERVICE IB & ensemble I & ensemble II \\
\hline plats & 53 & 168 \\
\hline assiettes & 913 & 887 \\
\hline bols & 4502 & 1868 \\
\hline total & 5468 & 2923 \\
\hline
\end{tabular}

ensemble I

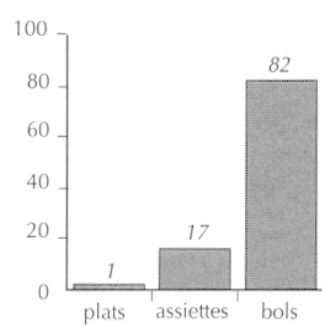

ensemble II

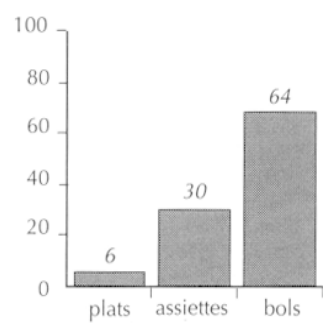

Fig. 34. Ensembles I et II, service IB, pourcentages respectifs des trois groupes morphologiques : plats, assiettes, bols.

\begin{tabular}{|l|c|c|}
\hline SERVICE II & ensemble I & ensemble II \\
\hline plats & 0 & 127 \\
\hline assiettes & 225 & 292 \\
\hline bols & 937 & 1236 \\
\hline total & $\mathbf{1 1 6 2}$ & $\mathbf{1 6 5 5}$ \\
\hline
\end{tabular}

ensemble I

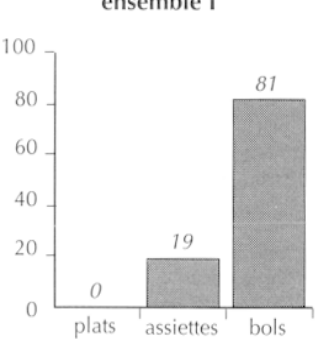

ensemble II

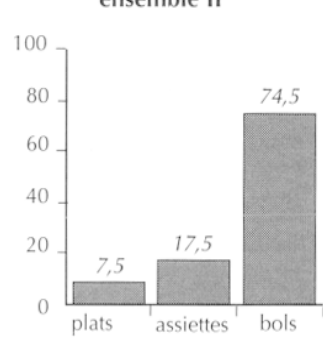

Fig. 36. Ensembles I et II, service II, représentation respective des trois groupes morphologiques : plats, assiettes, bols.

site dont l'approvisionnement est, pour autant que l'on sache, en grande partie d'origine lyonnaise, et dont l'occupation se situe dans les années 7 avant J.-C./9 après J.-C.

L'étude des céramiques sigillées permet donc tout d'abord, et le fait est nouveau, de distinguer deux périodes d'activité de l'atelier au sein de l'époque augustéenne.

\begin{tabular}{|l|c|c|}
\hline SERVICE IC & ensemble I & ensemble II \\
\hline plats & 83 & 607 \\
\hline assiettes & 2340 & 4508 \\
\hline bols & 1016 & 400 \\
\hline total & 3439 & 5515 \\
\hline
\end{tabular}

ensemble

ensemble II
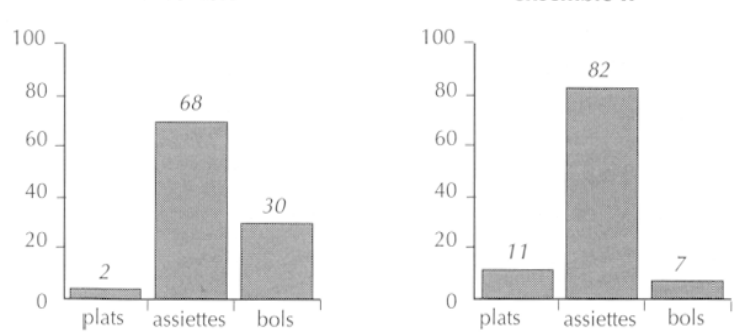

Fig. 35. Ensembles I et II, service IC, représentation respective des trois groupes morphologiques : plats, assiettes, bols.

Elle pose ensuite une nouvelle fois la question de savoir ce que recouvre encore la notion de service dans la vaisselle de table de l'époque. En effet, si l'on examine le service I dans sa globalité, on voit que les assiettes et les bols y sont représentés de façon équivalente, soit par $44 \%$ et $54,5 \%$ des objets mais on constate un très net décalage numérique à l'intérieur des services IB et IC entre les assiettes et les bols : le groupe morphologique des assiettes y est dominé par les assiettes IC ( $68 \%$ et $82 \%)$ et celui des bols par les bols IB ( $82 \%$ et $64 \%$ ) (fig. 34 et 35 ). Ce décalage attesté également sur les sites de consommation n'existerait pas s'il y avait eu, à la source, une quelconque intention de fabriquer, et donc de vendre, des "services de table " au sens traditionnel du terme, c'est-àdire des assortiments d'objets ayant diverses fonctions mais présentant tous la même facture et la même provenance (lieu et cachet de fabrication).

Le décalage assiettes/bols au sein du service II pris seul (fig. 36) ne revêt pas la même signification dans la mesure où on ne connaît pas précisément les différents usages affectés à tel ou tel groupe de formes. La notion de service est ici battue en brèche par un autre biais : l'étude typologique fine de ces vases révèle en effet une grande diversité dans les profils de bords et un manque total d'adéquation entre les bords des assiettes et ceux des bols. Cette question fondamentale, sur laquelle un matériel d'atelier jette un éclairage particulièrement intéressant, sera développée au terme de l'étude des timbres de potiers. 
L'analyse fine de ce mobilier soulève enfin nombre de questions sur la destination réelle de la production précoce qui reste en grande partie absente des ensembles du limes ou des sites de consommation urbains tels que les sites lyonnais eux-mêmes : pour qui a-t-on lancé cette production? Quels étaient et où étaient les marchés visés et la clientèle potentielle de l'atelier au début de son activité? La production que nous appellerons "classique " par opposition à la production " précoce " pose aussi, bien qu'en moindre part, le même problème d'interprétation : à quoi correspondent, par exemple, les assiettes $\mathrm{IB}$ « type Lyon " à la fois particulièrement homogènes et très abondantes durant cette seconde période mais qu'on ne retrouve qu'en proportions infimes sur le limes?

\section{LES TIMBRES}

Le catalogue publié en 1976 (Lasfargues, 1976) présentait un classement des timbres en quarante-deux grandes marques au sein desquelles apparaissaient les différentes graphies reconnues pour chacune de ces marques. Nous avons choisi de garder la même numérotation et le même mode de présentation afin de faciliter la lecture et la confrontation, le cas échéant, des deux documents.

Les dix contextes ont livré 2263 timbres dont 1247 pour l'ensemble I et 1016 pour l'ensemble II (fig. 37). Sur quarante-deux marques publiées en 1976, dix-neuf seulement sont attestées dans le mobilier des ensembles I et II, ce qui peut à priori surprendre. La relecture du catalogue montre cependant que les séries absentes de nos contextes sont, à une exception près (XXI, I.C.E.), notées comme " assez rares ", " très rares " ou " uniques ». On peut donc conférer une valeur représentative au corpus sur lequel nous avons travaillé et raisonner sur la base de données statistiques ayant toute chance d'être fiables.

\section{LES TIMBRES ET LA CHRONOLOGIE}

Le corpus, pris globalement, révèle tout d'abord que quatre marques sur dix-neuf rassemblent $75 \%$ du total des timbres (fig. 38).

La distribution par ensemble révèle cependant des différences notables qu'une étude précise des graphies permet de mettre en évidence (fig. 39 et 40).

La marque ATTIVS, qui représente $52 \%$ des timbres de l'ensemble I, observe une chute brutale dans l'ensemble II où elle se réduit à $4,6 \%$ du lot ; encore doit-on préciser que les ATTIVS de l'ensemble II (47 ex.) sont

\begin{tabular}{|c|c|c|c|}
\hline$N^{\circ}$ cat. & marques & ensemble & $\begin{array}{c}\text { ensemble } \\
\text { II }\end{array}$ \\
\hline III & ALPVS & 6 & 32 \\
\hline V & C.ATILLIVS & 0 & 1 \\
\hline VI & C.ATTIVS & 558 & 47 \\
\hline IX & CALAMVS & 1 & 0 \\
\hline XII & FAVSTVS & 5 & 1 \\
\hline $\mathrm{XVI}$ & FRONTO & 68 & 44 \\
\hline$X V I I$ & GAMVS & 6 & 2 \\
\hline$X I X$ & HILARVS & 2 & 0 \\
\hline XXIII & T.M.FORTVNATVS & 25 & 95 \\
\hline$X X V I I$ & RASINIVS & 165 & 128 \\
\hline$X X V I I I$ & RVSIICVS & 6 & 72 \\
\hline XXIX & C.SENTIVS & 330 & 102 \\
\hline$X X X$ & STABILIO & 0 & 1 \\
\hline XXXIII & L.THYRSVS & 26 & 344 \\
\hline XXXIV & TIGRANVS & 8 & 101 \\
\hline$X X X V$ & TVLLIVS & 28 & 0 \\
\hline XXXVIII & VARIVS & 0 & 1 \\
\hline XLI & VLON ou ELON & 13 & 43 \\
\hline \multirow[t]{2}{*}{$\mathrm{XI} I \mathrm{I}$} & anépigraphes & 0 & 2 \\
\hline & total & 1247 & 1016 \\
\hline
\end{tabular}

Fig. 37. Répartition des timbres par ensemble.

\begin{tabular}{|l|c|}
\hline \multicolumn{1}{|c|}{ marques } & unités \\
\hline C.ATTIVS VI & 605 \\
\hline RASINIVS XXVII & 293 \\
\hline C.SENTIVS XXIX & 432 \\
\hline L.THYRSVS XXXIII & 370 \\
\hline total & 1700 \\
\hline
\end{tabular}

Fig. 38. Représentation respective des quatre marques majoritaires dans les deux ensembles. 


\begin{tabular}{|l|r|}
\hline \multicolumn{1}{|c|}{ marques } & unités \\
\hline C.ATTIVS VI & 558 \\
\hline RASINIVS XXVII & 165 \\
\hline C.SENTIVS XXIX & 330 \\
\hline L.THYRSVS XXXIII & 26 \\
\hline total & 1079 \\
\hline
\end{tabular}

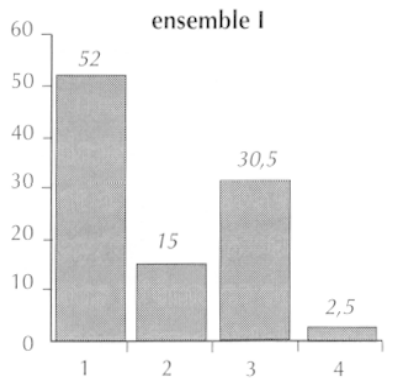

Fig. 39. Représentation respective des quatre marques majoritaires dans l'ensemble I.

\begin{tabular}{|l|c|}
\hline \multicolumn{1}{|c|}{ marques } & unités \\
\hline C.ATTIVS VI & 47 \\
\hline RASINIVS XXVII & 128 \\
\hline C.SENTIVS XXIX & 102 \\
\hline L.THYRSVS XXXIII & 344 \\
\hline total & $\mathbf{6 2 1}$ \\
\hline
\end{tabular}

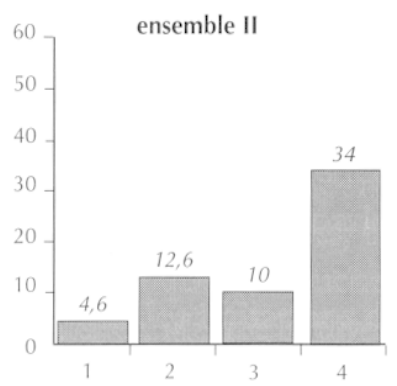

Fig. 40. Représentation respective des quatre marques majoritaires dans l'ensemble II.

tous imprimés sur plats et assiettes et possèdent, pour quarante et un d'entre eux, la même graphie (graphie 11).

La marque SENTIVS passe, quant à elle, de $30,5 \%$ à $10 \%$ du total de chaque ensemble.

À l'inverse, la marque THYRSVS qui ne comprend que $2 \%$ des timbres de l'ensemble I augmente jusqu'à $34 \%$ dans l'ensemble II.

Les pourcentages des timbres de RASINIVS, bien que similaires, rendent compte de deux faciès complètement différents. Parmi 165 RASINIVS de l'ensemble I, cent cinquante-cinq sont circulaires et imprimés exclusivement sur des bols (graphies 5-6) : ces graphies ne figurent plus ou presque dans l'ensemble II où cent vingt-six des cent vingt-huit timbres sont rectangulaires et imprimés cette fois exclusivement sur des assiettes (graphie 1).

Les observations que l'on peut faire sur les autres marques ne sauraient être exploitées telles quelles quand elles s'avèrent trop faiblement représentées : aussi n'ai-je retenu pour cette discussion que les séries et graphies comprenant un minimum de dix exemplaires dans l'un ou l'autre ensemble.
On observe tout d'abord que la marque TVLLIVS, qui va jusqu'à dépasser celle de THYRSVS dans l'ensemble I, est totalement absente dans l'ensemble II. Parallèlement, cinq marques, d'ailleurs imprimées presque exclusivement sur des bols (cf. infra, fig. 49) voient leurs effectifs se multiplier dans l'ensemble II :

- ALPVS (de 6 à 32 ex., soit de $0,5 \%$ à $3,2 \%$ ),

- T. MALIVS FORTVNATVS (de 25 à 95 ex., soit de $2 \%$ à $9,4 \%$ ),

- RVSTICVS (de 6 à 72 ex., soit de $0,5 \%$ à $7,1 \%$ ),

- TIGRANVS (de 8 à 100 ex., soit de 0,6\% à 9,8\%),

- VLON (de 11 à 43 ex., soit de $0,9 \%$ à 4,2\%).

L'étude fine des graphies révèle d'autres différences. La marque FRONTO, à peu près équivalente en nombre de timbres sur les deux ensembles, montre l'inversion de deux graphies : la graphie 2 , tout d'abord majoritaire ( 57 ex. sur 68), est ensuite remplacée par la graphie 4 dans l'ensemble II (41 ex. sur 44), sans qu'il y ait pour cela, comme c'est le cas pour RASINIVS, changement de support, puisque les timbres FRONTO sont tous imprimés sur des bols (cf. infra, fig. 49 et 50 ).

Ce premier examen du corpus fait ressortir entre les deux ensembles un certain nombre de discordances qui se traduisent soit par la disparition d'une marque, soit par un déséquilibre numérique important entre les marques et, parfois, entre les graphies d'une même marque. De telles données tendent à confirmer qu'on a bien affaire à deux phases de production mais indiquent également ce que suggérait déjà l'étude des formes, à savoir une continuité probable entre ces phases.

La confrontation de ces timbres avec ceux mis au jour à Haltern souligne un grand nombre d'absences sur les deux sites et prouve une nouvelle fois que nous sommes loin de connaître l'importance et le volume exacts des productions augustéennes de Lyon lato sensu (fig. 41). Elle a du moins le mérite de montrer que quelques marques et graphies abondantes dans l'ensemble II (notamment T. M. FORTVNATVS, L. THYRSVS, TIGRANVS) sont relativement bien représentées à Haltern. Il est clair, cependant, qu'elle n'apporte pas de renseignements plus précis que ceux fournis par l'étude typologique et statistique des vases.

\section{LES NOTIONS DE SPÉCIALISATION ET DE STANDARDISATION}

Cet ensemble de timbres, qu'il soit pris de façon globale ou par période, permet d'aborder des problèmes spécifiquement liés à l'organisation de la production au 


\begin{tabular}{|c|c|}
\hline marques & graphies \\
\hline \multirow[t]{2}{*}{ C.ATTIVS } & $\mathrm{VI}, 1$ \\
\hline & $\mathrm{VI}, 11$ \\
\hline FAVSTVS & XII, 1 \\
\hline \multirow[t]{2}{*}{ FRONTO } & $\mathrm{XVI}, 4$ \\
\hline & $X V I, 5$ \\
\hline GAMVS & $\mathrm{XVII}, 1$ \\
\hline \multirow[t]{2}{*}{ T.M.FORTVNATVS } & XXIII, 1 \\
\hline & XXIII, 2 \\
\hline \multirow[t]{2}{*}{ RASINIVS } & $\mathrm{XXVII}, 1$ \\
\hline & XXVII, 7 \\
\hline RVSTICVS & XXVIII, 1 \\
\hline \multirow[t]{2}{*}{ C.SENTIVS } & XXIX, 3 \\
\hline & XXIX, 9 \\
\hline \multirow[t]{6}{*}{ L.THYRSVS } & XXXIII, 1 \\
\hline & XXXIII, 2 \\
\hline & XXXIII, 5 \\
\hline & XXXIII, 7 \\
\hline & XXXIII, 17 \\
\hline & XXXIII, 19 \\
\hline TIGRANVS & XXXIV, 1 \\
\hline
\end{tabular}

Fig. 41. Craphies des deux ensembles attestés à Haltern.

sein de l'atelier : spécialisation des potiers, fonctionnement interne du travail, validité de la notion de "service ". Il faut donc dans un premier temps examiner, cas par cas, la distribution des marques ainsi que des graphies, sur assiettes et sur bols, et, dans un deuxième temps, la répartition de ces marques et graphies sur bols du service I et II. Seuls sont retenus les exemples tirés de lots comprenant au moins dix exemplaires.

\section{La distribution par forme, assiette/bol}

Si aucune marque n'apparaît exclusivement sur des assiettes, c'est le cas en revanche pour certaines graphies (fig. 42).

Six marques apparaissent exclusivement sur des bols (fig. 43).

\begin{tabular}{|l|c|c|c|}
\hline & ensemble I & ensemble II & total \\
\hline C.ATTIVS VI, 11 & 12 & 41 & 53 \\
\hline RASINIVS XXVII, 1 & 9 & 126 & 135 \\
\hline C.SENTIVS XXIX,9 & 10 & 77 & 87 \\
\hline L.T(H)YRSVS XXXIII, 14 & 0 & 40 & 40 \\
\hline L.T(H)YRSVS XXXIII, 15 & 0 & 14 & 14 \\
\hline L.T(H)YRSVS XXXIII, 16 & 3 & 28 & 31 \\
\hline L.T(H)YRSVS XXXIII, 17 & 9 & 113 & 122 \\
\hline total & 43 & 439 & 482 \\
\hline
\end{tabular}

Fig. 42. Graphies apparaissant exchusivement sur des assiettes.

\begin{tabular}{|l|r|r|r|}
\hline & ensemble I & ensemble II & total \\
\hline ALPVS III & 6 & 32 & 38 \\
\hline FRONTO XVI & 68 & 44 & 112 \\
\hline T.M.FORTVNATVS XXIII & 25 & 95 & 120 \\
\hline RVSTICVS XXVIII & 6 & 72 & 78 \\
\hline TVLLIVS XXXV & 28 & 0 & $\mathbf{2 8}$ \\
\hline VLON XLI & 13 & 43 & $\mathbf{5 6}$ \\
\hline total & 146 & 286 & 432 \\
\hline
\end{tabular}

Fig. 43. (maphies apparaissant exclusivement sur des bols.

Neuf graphies de marques attestées sur assiettes et sur bols apparaissent exclusivement sur des bols (fig. 44).

\section{La distribution par service (fig. 45 à 50$)$}

Les fréquences des marques et graphies sur plats et assiettes du service I ou du service II ne peuvent être déterminées en raison de la fragmentation du matériel qui interdit la plupart du temps d'attribuer tel ou tel fond à l'un des deux services. On note du moins que la graphie 10 de C. SENTIVS est attestée dans l'ensemble I aussi bien sur des assiettes du service I (série IB/IC, inv. : 26, 27, 422, 528) que sur des assiettes précoces du service II (groupe 1, inv. : $7,487,488)$; cette graphie est en l'occurrence celle qui a livré les deux seuls timbres radiaux comnus sur l'atelier et imprimés sur des plats ou assiettes de types 


\begin{tabular}{|l|r|r|r|}
\hline & ensemble I & ensemble II & total \\
\hline C.ATTIVS VI, 8 & 15 & 0 & 15 \\
\hline C.ATTIVS VI, 9 & 32 & 0 & 32 \\
\hline C.ATTIVS VI, 10 & 27 & 0 & 27 \\
\hline RASINIVS XXVII, 5-6 & 155 & 1 & 156 \\
\hline L.THYRSVS XXXIII, 4 & 12 & 22 & 34 \\
\hline L.THYRSVS XXXIII, 5 & 0 & 11 & 11 \\
\hline L.THYRSVS XXXIII, 8 & 0 & 74 & 74 \\
\hline TIGRANVS XXXIV, 1 & 8 & 95 & 103 \\
\hline total & 249 & 203 & 452 \\
\hline
\end{tabular}

Fig. 44. Graphies (de marques attestées sur assiettes et sur bols) apparaissant exclusivement sur des bols.

\begin{tabular}{|l|r|r|c|}
\hline & \multicolumn{1}{|c|}{ S.I } & \multicolumn{1}{c|}{ S.II } & indét. \\
\hline FRONTO XVI & 105 & 1 & 6 \\
\hline RASINIVS XXVII & 141 & 5 & 11 \\
\hline TVLLIVS XXXV & 23 & 0 & 5 \\
\hline VLON XLI & 41 & 0 & 14 \\
\hline total & 310 & 6 & 36 \\
\hline
\end{tabular}

Fig. 45. Marques majoritaires sur les bols du service I.

\begin{tabular}{|l|r|r|r|}
\hline & \multicolumn{1}{|c|}{ S.I } & \multicolumn{1}{|c|}{ S.II } & indét. \\
\hline C.ATTIVS VI, 1 & 33 & 0 & 36 \\
\hline C.ATTIVS VI, 3 & 61 & 4 & 28 \\
C.ATTIVS VI, 8 & 12 & 0 & 3 \\
C.ATTIVS VI, 9 & 23 & 2 & 7 \\
\hline C.SENTIVS XXIX, 2 & 141 & 1 & 18 \\
\hline C.SENTIVS XXIX, 4 & 39 & 1 & 9 \\
\hline L.THYRSVS XXXIII, 4 & 31 & 2 & 1 \\
\hline L.THYRSVS XXXIII, 8 & 67 & 4 & 3 \\
\hline total & 407 & 14 & 105 \\
\hline
\end{tabular}

Fig. 46. Graphies majoritaires sur les bols du service I. indéterminés. L'étude de la distribution par service se fonde par conséquent sur les formes de bols.

\section{Timbres sur bols du service I}

En dépit de la présence de fonds représentés par des fragments qui empêchent toute identification précise, on note que quatre grandes marques apparaissent le plus souvent sur des bols du service I : FRONTO, RASINIVS; TVLIIVS, VLON (fig. 45).

Huit graphies sont ensuite en majorité sur service I (fig. 46).

\section{Timbres sur bols du service II}

Deux marques sont attestées de façon presque systématique sur des bols du service II (fig. 47).

Deux graphies de C. ATTIVS et une graphie de C. SENTIVS sont majoritaires sur des bols du service II (fig. 48).

Il est donc patent que sept marques représentent autant de cachets de fabrication réservés aux bols, voire, et c'est là tout le problème, autant de potiers spécialisés dans la production de bols : ALPVS, FRONTO, T. MALIVS FORTVNATVS, RVSTICVS, TVLLIVS, VLON (fig. 49 et 50). Pour ces marques, la répartition service $\mathrm{I} /$ service II ne change pas de l'un à l'autre ensemble :

\begin{tabular}{|l|c|c|c|}
\hline & S.I & S.II & indét. \\
\hline ALPVS III & 1 & 34 & 3 \\
\hline T.M.FORTVNATVS XXIII & 0 & 119 & 1 \\
\hline total & 1 & 153 & 4 \\
\hline
\end{tabular}

Fig. 47. Marques majoritaires sur les bols du service II.

\begin{tabular}{|l|c|c|c|}
\hline & S.I & S.II & indét. \\
\hline C.ATTIVS VI, 6 & 9 & 32 & 19 \\
\hline C.ATTIVS VI, 10 & 5 & 16 & 6 \\
\hline C.SENTIVS XXIX, 3 & 1 & 15 & 1 \\
\hline total & 15 & 63 & $\mathbf{2 6}$ \\
\hline
\end{tabular}

Fig. 48. Gmphies majoritaires sur les bols du service II. 


\begin{tabular}{|c|c|c|c|c|}
\hline $\mathrm{N}^{\circ}$ cat. & marques & formes & $\begin{array}{c}\text { ensemble } \\
1\end{array}$ & $\begin{array}{c}\text { ensemble } \\
\text { II }\end{array}$ \\
\hline \multirow[t]{2}{*}{ III } & \multirow[t]{2}{*}{ ALPVS } & assiettes & 0 & 0 \\
\hline & & bols & 6 & 32 \\
\hline \multirow[t]{2}{*}{ V } & \multirow[t]{2}{*}{ C.ATILLIVS } & assiettes & 0 & 1 \\
\hline & & bols & 0 & 0 \\
\hline \multirow[t]{2}{*}{ VI } & \multirow[t]{2}{*}{ C.ATTIVS } & assiettes & 236 & 47 \\
\hline & & bols & 322 & 0 \\
\hline \multirow[t]{2}{*}{ IX } & \multirow[t]{2}{*}{ CALAMVS } & assiettes & 0 & 0 \\
\hline & & bols & 1 & 0 \\
\hline \multirow[t]{2}{*}{ XII } & \multirow[t]{2}{*}{ FAVSTVS } & assiettes & 0 & 0 \\
\hline & & bols & 5 & 1 \\
\hline \multirow[t]{2}{*}{ XVI } & \multirow[t]{2}{*}{ FRONTO } & assiettes & 0 & 0 \\
\hline & & bols & 68 & 44 \\
\hline \multirow[t]{2}{*}{ XVII } & \multirow[t]{2}{*}{ GAMVS } & assiettes & 1 & 0 \\
\hline & & bols & 5 & 2 \\
\hline \multirow[t]{2}{*}{ XIX } & \multirow[t]{2}{*}{ HILARVS } & assiettes & 2 & 0 \\
\hline & & bols & 0 & 0 \\
\hline \multirow[t]{2}{*}{ XXIII } & \multirow[t]{2}{*}{ T.M.FORTVNATVS } & assiettes & 0 & 0 \\
\hline & & bols & 25 & 95 \\
\hline \multirow[t]{2}{*}{ XXVII } & \multirow[t]{2}{*}{ RASINIVS } & assiettes & 10 & 126 \\
\hline & & bols & 155 & 2 \\
\hline \multirow[t]{2}{*}{ XXVIII } & \multirow[t]{2}{*}{ RVSTICVS } & assiettes & 0 & 0 \\
\hline & & bols & 6 & 72 \\
\hline \multirow[t]{2}{*}{ XXIX } & \multirow[t]{2}{*}{ C.SENTIVS } & assiettes & 100 & 84 \\
\hline & & bols & 230 & 18 \\
\hline \multirow[t]{2}{*}{$X X X$} & \multirow[t]{2}{*}{ STABILIO } & assiettes & 0 & 0 \\
\hline & & bols & 0 & 1 \\
\hline \multirow[t]{2}{*}{ XXXIII } & L.THYRSVS & assiettes & 13 & 216 \\
\hline & & bols & 13 & 128 \\
\hline XXXIV & TIGRANVS & assiettes & 0 & 1 \\
\hline & & bols & 8 & 100 \\
\hline XXXV & TVLLIVS & assiettes & 0 & 0 \\
\hline & & bols & 28 & 0 \\
\hline XXXVIII & SEX.VARIVS & assiettes & 0 & 0 \\
\hline & & bols & 0 & 1 \\
\hline XLI & VLON & assiettes & 0 & 0 \\
\hline & & bols & 13 & 43 \\
\hline XLIII & anépigraphes & assiettes & 0 & 0 \\
\hline & & bols & 0 & 2 \\
\hline & total & & 1247 & 1016 \\
\hline
\end{tabular}

Fig. 49. Répartition par ensemble des marques sur les assiettes et les bols. prenons, pour exemple, le cas de T. MALIVS FORTVNATVS qui signe toujours sur service II, que ce soit dans l'ensemble I où ce service rassemble à peine $10 \%$ des vases sigillés ou bien dans l'ensemble II où ses effectifs augmentent légèrement; on notera d'ailleurs que les timbres de cette marque semblent "réservés " aux bols du groupe 2.

\section{SYNTHÈSE}

\section{La notion de service}

Aux observations déjà énoncées au terme de l'étude typologique et statistique des vases, s'ajoutent celles que l'on peut faire grâce à cet ensemble de timbres et qui concordent en grande partie avec les hypothèses émises en 1982 par S. von Schnurbein d'après les timbres de Haltern.

Il est clair que les potiers Alpvs, Fronto, Titvs Malivs Fortvnatvs, Rvsticvs, Tvllivs et Vlon ne se sont jamais lancés dans la fabrication et la diffusion de plats et d'assiettes. De plus, on a vu que, selon les cas, certains étaient spécialisés dans le service I ou dans le service II et que d'autres ont signé des bols des deux services (fig. 51).

Les potiers qui ont abondamment produit et diffusé aussi bien des assiettes que des bols et, pour les bols, aussi bien des formes du service I que du service II sont les quatre qui représentent l'essentiel de l'effectif des timbres dans les deux ensembles : C. Attivs, Rasinivs, C. Sentivs, L. Thyrsvs (fig. 52 à 54). La présence de poinçons identiques sur assiettes ou bols de différents types, ainsi que sur bols du service I ou du service II à l'intérieur de ces grandes marques, tend à confirmer que les potiers qui avaient inscrit les principaux types d'assiettes et de bols à leur répertoire n'ont pas voulu fabriquer de "services " à proprement parler. Or, si rien ne les en empêchait sur le plan technique et pratique, il nous faut bien supposer qu'il n'y avait tout simplement pas de " demande " de la part d'une clientèle moins préoccupée qu'on ne le suppose d'assortir les vases destinés au service de la table. De toute façon, si on persistait à considérer cette notion de "service " au sens le plus strict du terme (assortiment d'objets présentant les mêmes caractéristiques typologiques et le même " cachet " de fabrication), on ferait fi de ce que montre le matériel de l'atelier d'une part et celui des sites de consommation d'autre part, à savoir des déséquilibres numériques constants au sein du service I entre assiettes et bols IB ainsi qu'entre assiettes et bols IC 


\begin{tabular}{|c|c|c|c|c|c|c|}
\hline & \multicolumn{3}{|c|}{ assiettes } & \multicolumn{3}{|c|}{ bols } \\
\hline & $\begin{array}{c}\text { ensemble } \\
\text { I }\end{array}$ & $\begin{array}{c}\text { ensemble } \\
\text { II }\end{array}$ & total & $\begin{array}{c}\text { ensemble } \\
1\end{array}$ & $\begin{array}{c}\text { ensemble } \\
\text { II }\end{array}$ & total \\
\hline ALPVS III, 1 & 0 & 0 & 0 & 6 & 32 & 38 \\
\hline total & 0 & 0 & 0 & 6 & 32 & 38 \\
\hline C.ATILLIVS V, 1 & 0 & 1 & 1 & 0 & 1 & 0 \\
\hline C.ATTIVS VI, 1 & 130 & 5 & 135 & 69 & 0 & 69 \\
\hline C.ATTIVS VI, 2 & 0 & 1 & 1 & 0 & 0 & 0 \\
\hline C.ATTIVS VI, 3 & 21 & 0 & 21 & 93 & 0 & 93 \\
\hline C.ATTIVS VI, 4 & 5 & 0 & 5 & 6 & 0 & 6 \\
\hline C.ATTIVS VI, 5 & 56 & 0 & 56 & 20 & 0 & 20 \\
\hline C.ATTIVS VI, 6 & 12 & 0 & 12 & 60 & 0 & 60 \\
\hline C.ATTIVS VI, 8 & 0 & 0 & 0 & 15 & 0 & 15 \\
\hline C.ATTIVS VI, 9 & 0 & 0 & 0 & 32 & 0 & 32 \\
\hline C.ATTIVS VI, 10 & 0 & 0 & 0 & 27 & 0 & 27 \\
\hline C.ATTIVS VI, 11 & 12 & 41 & 53 & 0 & 0 & 0 \\
\hline total & 236 & 47 & 283 & 322 & 0 & 322 \\
\hline CALAMVS IX, 1 & 0 & 0 & 0 & 1 & 0 & 1 \\
\hline FAVSTVS XII, 1 & 0 & 0 & 0 & 5 & 1 & 6 \\
\hline FRONTO XVI, 1 & 0 & 0 & 0 & 4 & 0 & 4 \\
\hline FRONTO XVI, 2 & 0 & 0 & 0 & 57 & 1 & 58 \\
\hline FRONTO XVI, 4 & 0 & 0 & 0 & 5 & 41 & 46 \\
\hline FRONTO XVI, 5 & 0 & 0 & 0 & 1 & 2 & 3 \\
\hline FRONTO XVI, 6 & 0 & 0 & 0 & 1 & 0 & 1 \\
\hline total & 0 & 0 & 0 & 68 & 44 & 112 \\
\hline GAMUS XVII, 1 & 1 & 0 & 1 & 2 & 2 & 4 \\
\hline GAMUS XVIII, 2 & 0 & 0 & 0 & 3 & 0 & 3 \\
\hline HILARVS XIX & 2 & 0 & 2 & 0 & 0 & 0 \\
\hline T.M.FORTVNATVS XXIII, 1 & 0 & 0 & 0 & 24 & 91 & 115 \\
\hline T.M.FORTVNATVS XXIII, 2 & 0 & 0 & 0 & 1 & 4 & 5 \\
\hline total & 0 & 0 & 0 & 25 & 95 & 120 \\
\hline RASINIVS XXVII, 1 & 9 & 126 & 135 & 0 & 0 & 0 \\
\hline RASINIVS XXVII, 5 & 0 & 0 & 0 & 152 & 1 & 153 \\
\hline RASINIVS XXVII, 6 & 0 & 0 & 0 & 3 & 0 & 3 \\
\hline RASINIVS XXVII, 7 & 1 & 0 & 1 & 0 & 1 & 1 \\
\hline total & 10 & 126 & 136 & 155 & 2 & 157 \\
\hline RVSTICVS XXVIII, 1 & 0 & 0 & 0 & 6 & 69 & 75 \\
\hline RVSTICVS XXVIII, 2 & 0 & 0 & 0 & 0 & 3 & 3 \\
\hline total & 0 & 0 & 0 & 6 & 72 & 78 \\
\hline
\end{tabular}

Fig. 50. Répartition par ensemble des graphies sur les assiettes et les bols.

\begin{tabular}{|c|c|c|c|c|c|c|}
\hline & \multicolumn{3}{|c|}{ assiettes } & \multicolumn{3}{|c|}{ bols } \\
\hline & $\begin{array}{c}\text { ensemble } \\
1\end{array}$ & $\begin{array}{c}\text { ensemble } \\
\text { II }\end{array}$ & total & $\begin{array}{c}\text { ensemble } \\
1\end{array}$ & $\begin{array}{c}\text { ensemble } \\
\text { II }\end{array}$ & total \\
\hline C.SENTIVS XXIX, 1 & 2 & 0 & 2 & 0 & 0 & 0 \\
\hline C.SENTIVS XXIX, 2 & 10 & 0 & 10 & 156 & 4 & 160 \\
\hline C.SENTIVS XXIX, 3 & 0 & 1 & 1 & 3 & 14 & 17 \\
\hline C.SENTIVS XXIX, 4 & 4 & 0 & 4 & 49 & 0 & 49 \\
\hline C.SENTIVS XXIX, 5 & 1 & 0 & 1 & 1 & 0 & 1 \\
\hline C.SENTIVS XXIX, 6 & 1 & 0 & 1 & 10 & 0 & 10 \\
\hline C.SENTIVS XXIX, 7 & 2 & 1 & 3 & 4 & 0 & 4 \\
\hline C.SENTIVS XXIX, 8 & 3 & 0 & 3 & 0 & 0 & 0 \\
\hline C.SENTIVS XXIX, 9 & 10 & 77 & 87 & 0 & 0 & 0 \\
\hline C.SENTIVS XXIX, 10 & 67 & 1 & 68 & 7 & 0 & 7 \\
\hline C.SENTIVS XXIX, 12 & 0 & 3 & 3 & 0 & 0 & 0 \\
\hline C.SENTIVS XXIX, 13 & 0 & 1 & 1 & 0 & 0 & 0 \\
\hline total & 100 & 84 & 184 & 230 & 18 & 248 \\
\hline STABILIO XXX & 0 & 0 & 0 & 0 & 1 & 1 \\
\hline L.THYRSVS XXXIII, 1 & 1 & 0 & 1 & 0 & 0 & 0 \\
\hline L.THYRSVS XXXIII, 2 & 0 & 9 & 9 & 0 & 0 & 0 \\
\hline L.THYRSVS XXXIII, 3 & 0 & 3 & 3 & 0 & 2 & 2 \\
\hline L.THYRSVS XXXIII, 4 & 0 & 0 & 0 & 12 & 21 & 33 \\
\hline L.THYRSVS XXXIII, 5 & 0 & 0 & 0 & 0 & 12 & 12 \\
\hline L.THYRSVS XXXIII, 6 & 0 & 0 & 0 & 0 & 1 & 1 \\
\hline L.THYRSVS XXXIII, 7 & 0 & 0 & 0 & 1 & 0 & 1 \\
\hline L.THYRSVS XXXIII, 8 & 0 & 0 & 0 & 0 & 74 & 74 \\
\hline L.THYRSVS XXXIII, 9 & 0 & 2 & 2 & 0 & 0 & 0 \\
\hline L.THYRSVS XXXIII, 10 & 0 & 0 & 0 & 0 & 8 & 8 \\
\hline L.THYRSVS XXXIII, 12 & 0 & 0 & 0 & 0 & 1 & 1 \\
\hline L.THYRSVS XXXIII, 13 & 0 & 0 & 0 & 0 & 6 & 6 \\
\hline L.THYRSVS XXXIII, 14 & 0 & 40 & 40 & 0 & 0 & 0 \\
\hline L.THYRSVS XXXIII, 15 & 0 & 14 & 14 & 0 & 0 & 0 \\
\hline L.THYRSVS XXXIII, 16 & 3 & 28 & 31 & 0 & 0 & 0 \\
\hline L.THYRSVS XXXIII, 15/16 & 0 & 6 & 6 & 0 & 0 & 0 \\
\hline L.THYRSVS XXXIII, 17 & 9 & 113 & 122 & 0 & 0 & 0 \\
\hline L.THYRSVS XXXIII, 18 & 0 & 1 & 1 & 0 & 0 & 0 \\
\hline L.THYRSVS XXXIII, 19 & 0 & 0 & 0 & 0 & 3 & 3 \\
\hline total & 13 & 216 & 229 & 13 & 128 & 141 \\
\hline TIGRANVS XXXIV, 1 & 0 & 0 & 0 & 8 & 95 & 103 \\
\hline TIGRANVS XXXIV, 5 & 0 & 1 & 1 & 0 & 5 & 5 \\
\hline total & 0 & 1 & 1 & 8 & 100 & 108 \\
\hline TVLLIVS XXXV, 1 & 0 & 0 & 0 & 28 & 0 & 28 \\
\hline SEX.VARIVS XXXVIIII, 1 & 0 & 0 & 0 & 0 & 1 & 1 \\
\hline VLON XLI, 1 & 0 & 0 & 0 & 11 & 43 & 54 \\
\hline VLON XLI, 4 & 0 & 0 & 0 & 1 & 0 & 1 \\
\hline total & 0 & 0 & 0 & 12 & 43 & 55 \\
\hline
\end{tabular}




\begin{tabular}{|c|c|c|c|c|c|c|c|c|c|}
\hline & \multirow{2}{*}{\multicolumn{4}{|c|}{ ensemble I }} & \multirow{2}{*}{\multicolumn{4}{|c|}{ ensemble II }} \\
\hline & & & & & & & & & \\
\hline$N^{\circ}$ cat. & marques & S.I & S.II & indét. & total & S.I & S.II & indét. & total \\
\hline III, 1 & ALPVS & 1 & 5 & 0 & 6 & 0 & 29 & 3 & 32 \\
\hline $\mathrm{VI}$ & ATTIVS & 155 & 61 & 106 & 322 & 0 & 0 & 0 & 0 \\
\hline IX & CALAMVS & 0 & 0 & 1 & 1 & 0 & 0 & 0 & 0 \\
\hline XII & FAVSTVS & 3 & 0 & 2 & 5 & 1 & 0 & 0 & 1 \\
\hline XVI & FRONTO & 63 & 1 & 4 & 68 & 42 & 0 & 2 & 44 \\
\hline$X V I I$ & GAMVS & 3 & 0 & 2 & 5 & 0 & 0 & 1 & 1 \\
\hline XXIII & T.M.FORTVNATVS & 0 & 25 & 0 & 25 & 0 & 93 & 2 & 95 \\
\hline$X X V I I$ & RASINIVS & 140 & 5 & 10 & 155 & 1 & 0 & 1 & 2 \\
\hline XXVIII & RVSTICVS & 0 & 6 & 0 & 6 & 11 & 46 & 15 & 72 \\
\hline$X X I X$ & C.SENTIVS & 190 & 4 & 36 & 230 & 4 & 13 & 1 & 18 \\
\hline$X X X$ & STABILIO & 0 & 0 & 0 & 0 & 1 & 0 & 0 & 1 \\
\hline XXXIII & THYRSVS & 12 & 1 & 0 & 13 & 110 & 13 & 5 & 128 \\
\hline XXXIV & TIGRANVS & 6 & 1 & 1 & 8 & 55 & 4 & 41 & 100 \\
\hline$X X X V$ & M.TVLLIVS & 23 & 0 & 5 & 28 & 0 & 0 & 0 & 0 \\
\hline XXXVIII & S.VARIVS & 0 & 0 & 0 & 0 & 0 & 1 & 0 & 1 \\
\hline XLI & VLON & 10 & 0 & 2 & 12 & 31 & 1 & 11 & 43 \\
\hline \multirow[t]{2}{*}{ XLII } & anépigraphes & 0 & 0 & 0 & 0 & 0 & 2 & 0 & 2 \\
\hline & total & 606 & 109 & 169 & 884 & 256 & 202 & 82 & 540 \\
\hline
\end{tabular}

Fig. 51. Ensembles I et II, répartition des marques par service.

\begin{tabular}{|c|c|c|c|c|c|}
\hline $\mathrm{N}^{\circ}$ cat. & marques & S.I & S.II & indét. & total \\
\hline III & ALPVS & 1 & 34 & 3 & 38 \\
\hline VI & C.ATTIVS & 155 & 61 & 106 & 322 \\
\hline IX & CALAMVS & 0 & 0 & 1 & 1 \\
\hline XII & FAVSTVS & 4 & 0 & 2 & 6 \\
\hline $\mathrm{XVI}$ & FRONTO & 105 & 1 & 6 & 112 \\
\hline XVII & GAMVS & 3 & 0 & 3 & 6 \\
\hline XXIII & T.M.FORTVNATVS & 0 & 118 & 2 & 120 \\
\hline XXVII & RASINIVS & 141 & 5 & 11 & 157 \\
\hline XXVIII & RVSTICVS & 11 & 53 & 14 & 78 \\
\hline XXIX & C.SENTIVS & 193 & 17 & 37 & 247 \\
\hline$x x x$ & STABILIO & 1 & 0 & 0 & 1 \\
\hline XXXIII & L.THYRSVS & 122 & 14 & 5 & 141 \\
\hline XXXIV & TIGRANVS & 62 & 5 & 42 & 109 \\
\hline$X X X V$ & TVLLIVS & 23 & 0 & 5 & 28 \\
\hline XXXVIII & SEX.VARIVS & 0 & 1 & 0 & 1 \\
\hline XLI & VLON & 41 & 0 & 14 & 55 \\
\hline \multirow[t]{2}{*}{ XLII } & anépigraphes & 0 & 3 & 0 & 3 \\
\hline & total & 862 & 312 & 251 & 1425 \\
\hline
\end{tabular}

(cf. supra). Mais admettre qu'un service ne se composait pas de pièces fabriquées à l'identique pose d'autres problèmes d'interprétation.

\section{L'organisation interne du travail}

Afin de constituer ce type d'assortiment, il fallait donc rassembler des vases signés par des potiers différents mais travaillant dans la même zone de production et probablement au contact des mêmes relais de diffusion : on avait par exemple quelques plats et assiettes de C. ATTIVS associés à des bols signés RASINIVS ou FRONTO, etc. Cette hypothèse suppose explicitement que les potiers s'organisaient dans le cadre d'une coopérative qui leur permettait de se compléter et (ou) de se relayer à différents stades du travail. Une fois défini tel ou tel " programme " de production par marque, ils se seraient ensuite associés au stade de la cuisson à l'image de ce que feront plus tard les potiers de la Graufesenque et comme en attestent les

Fig. 52. Répartition générale des marques par service. 


\begin{tabular}{|c|c|c|c|c|c|c|}
\hline \multirow{2}{*}{$\begin{array}{l}\text { marques } \\
\text { et } \\
\text { graphies }\end{array}$} & \multicolumn{3}{|c|}{ ensemble I } & \multicolumn{3}{|c|}{ ensemble II } \\
\hline & S.I & S.II & indét. & S.I & S.II & indét. \\
\hline ALPVS III, 1 & 1 & 5 & 0 & 0 & 29 & 3 \\
\hline ATTIVS VI, 1 & 33 & 0 & 36 & 0 & 0 & 0 \\
\hline ATTIVS VI, 3 & 61 & 4 & 28 & 0 & 0 & 0 \\
\hline ATTIVS VI, 4 & 2 & 0 & 4 & 0 & 0 & 0 \\
\hline ATTIVS VI, 5 & 10 & 7 & 3 & 0 & 0 & 0 \\
\hline ATTIVS VI, 6 & 9 & 32 & 19 & 0 & 0 & 0 \\
\hline ATTIVS VI, 8 & 12 & 0 & 3 & 0 & 0 & 0 \\
\hline ATTIVS VI, 9 & 23 & 2 & 7 & 0 & 0 & 0 \\
\hline ATTIVS VI, 10 & 5 & 16 & 6 & 0 & 0 & 0 \\
\hline total & 155 & 61 & 106 & 0 & 0 & 0 \\
\hline CALAMVS IX, 1 & 0 & 0 & 1 & 0 & 0 & 0 \\
\hline FAVSTVS XII, 1 & 3 & 0 & 2 & 1 & 0 & 0 \\
\hline FRONTO XVI, 1 & 2 & 1 & 1 & 0 & 0 & 0 \\
\hline FRONTO XVI, 2 & 55 & 0 & 2 & 1 & 0 & 0 \\
\hline FRONTO XVI, 4 & 5 & 0 & 0 & 39 & 0 & 2 \\
\hline FRONTO XVI, 5 & 0 & 0 & 1 & 2 & 0 & 0 \\
\hline FRONTO XVI, 6? & 1 & 0 & 0 & 0 & 0 & 0 \\
\hline total & 63 & 1 & 4 & 42 & 0 & 2 \\
\hline GAMVS XVII, 1 & 1 & 0 & 2 & 0 & 0 & 1 \\
\hline GAMVS XVII, 2 & 2 & 0 & 0 & 0 & 0 & 0 \\
\hline T.M.F. XXIII, 1 & 0 & 24 & 0 & 0 & 89 & 2 \\
\hline T.M.F. XXIII, 2 & 0 & 1 & 0 & 0 & 4 & 0 \\
\hline total & 0 & 25 & 0 & 0 & 93 & 2 \\
\hline RASINIVS XXVII, 5-6 & 140 & 5 & 10 & 1 & 0 & 0 \\
\hline RASINIVS XXVII, 7 & 0 & 0 & 0 & 0 & 0 & 1 \\
\hline total & 140 & 5 & 10 & 1 & $\mathbf{0}$ & 1 \\
\hline RVSTICVS XXVIII, 1 & 0 & 6 & 0 & 11 & 43 & 15 \\
\hline RVSTICVS XXVIII, 2 & 0 & 0 & 0 & 0 & 3 & 0 \\
\hline total & 0 & 6 & 0 & 11 & 46 & 15 \\
\hline
\end{tabular}

\begin{tabular}{|c|c|c|c|c|c|c|}
\hline \multirow{2}{*}{$\begin{array}{c}\text { marques } \\
\text { et } \\
\text { graphies }\end{array}$} & \multicolumn{3}{|c|}{ ensemble I } & \multicolumn{3}{|c|}{ ensemble II } \\
\hline & S.I & S.II & indét. & S.I & S.II & indét. \\
\hline C.SENTIVS XXIX, 2 & 137 & 1 & 18 & 4 & 0 & 0 \\
\hline C.SENTIVS XXIX, 3 & 1 & 2 & 0 & 0 & 13 & 1 \\
\hline C.SENTIVS XXIX, 4 & 39 & 1 & 9 & 0 & 0 & 0 \\
\hline C.SENTIVS XXIX, 5 & 1 & 0 & 0 & 0 & 0 & 0 \\
\hline C.SENTIVS XXIX, 6 & 9 & 0 & 1 & 0 & 0 & 0 \\
\hline C.SENTIVS XXIX, 7 & 3 & 0 & 1 & 0 & 0 & 0 \\
\hline C.SENTIVS XXIX, 10 & 0 & 0 & 7 & 0 & 0 & 0 \\
\hline total & 190 & 4 & 36 & 4 & 13 & 1 \\
\hline STABILIO XXX & 0 & 0 & 0 & 1 & 0 & 0 \\
\hline L.T(H)YRSVS XXXIII, 3 & 0 & 0 & 0. & 2 & 0 & 0 \\
\hline L.T(H)YRSVS XXXIII, 4 & 11 & 1 & 0 & 20 & 1 & 1 \\
\hline L.T(H)YRSVS XXXIII, 5 & 0 & 0 & 0 & 6. & 5 & 0 \\
\hline L.T(H)YRSVS XXXIII, 6 & 0 & 0 & 0 & 1 & 0 & 0 \\
\hline L.T(H)YRSVS XXXIII, 7 & 1 & 0 & 0 & 0 & 0 & 0 \\
\hline L.T(H)YRSVS XXXIII, 8 & 0 & 0 & 0 & 67 & 4 & 3 \\
\hline L.T(H)YRSVS XXXIII, 10 & 0 & 0 & 0 & 8 & 0 & 0 \\
\hline L.T(H)YRSVS XXXIII, 12 & 0 & 0 & 0 & 1. & 0 & 0 \\
\hline L.T(H)YRSVS XXXIII, 13 & 0 & 0 & 0 & 5 & 0 & 1 \\
\hline L.T(H)YRSVS XXXIII, 19 & 0 & 0 & 0 & 0 & 3 & 0 \\
\hline total & 12 & 1 & 0 & 110 & 13 & 5 \\
\hline C.TIGRANVS XXXIV, 1 & 6 & 1 & 1 & 50 & 4 & 40 \\
\hline C.TIGRANVS XXXIV, 5 & 0 & 0 & 0 & 5 & 0 & 1 \\
\hline total & 6 & 1 & 1 & 55 & 4 & 41 \\
\hline M.TVLLIVS XXXV, 1 & 23 & 0 & 5 & 0 & 0 & 0 \\
\hline SEX.VARIVS XXXVIII & 0 & 0 & 0 & 0 & 1 & 0 \\
\hline VLON XLI, 1 & 9 & 0 & 2 & 31 & 0 & 12 \\
\hline VLON XLI, 4? & 1 & 0 & 0 & 0 & 0 & 0 \\
\hline total & 10 & 0 & 2 & 31 & 0 & 12 \\
\hline anépigraphes & 0 & 0 & 0 & 0 & 2 & 0 \\
\hline
\end{tabular}

Fig. 53. Ensembles I et II, répartition des marques et graphies par service. comptes de cuisson mis au jour sur cet atelier. Ce type d'association entre les potiers de la Muette au stade de la cuisson semble logique, ne serait-ce que du point de vue de la rentabilité des fours : les potiers spécialisés dans des créneaux très étroits et représentant sans doute des petits ateliers ne pouvaient pourvoir individuellement à une seule et même fournée; ainsi « utilisés en commun » les fours devaient fonctionner plus souvent et à plein rendement. Il est tentant de supposer que cette pratique permettait également aux ateliers, qu'ils soient de taille modeste ou de plus grandes "manufactures ", de mieux planifier la production.
On peut donc imaginer qu'à l'étape suivante on ait procédé à des assortiments de vases de marques différentes en vue de leur commercialisation et en fonction de demandes précises. Le rôle des potiers eux-mêmes peut avoir différé selon qu'il s'agissait de petits ou de grands ateliers. Tenter de préciser ce rôle dans le cas de grandes fabriques comme celles de C. Attivs, Rasinivs, C. Sentivs ou L. Thyrsvs supposerait que l'on sache en amont qui dirigeait réellement ces fabriques. Quoiqu'il en soit, tout le problème est de savoir qui, et à quelle étape du travail, se chargeait de procéder à ces assortiments : les potiers s'arrangeaient-ils directement entre eux ou bien cette tâche revenait-elle à d'autres instances (marchands, gros- 


\begin{tabular}{|c|c|c|c|c|}
\hline marques & S.I & S.II & indét. & total \\
\hline ALPVS III, 1 & 1 & 34 & 3 & 38 \\
\hline C.ATTIVS VI, 1 & 33 & 0 & 36 & 69 \\
\hline C.ATTIVS VI, 3 & 61 & 4 & 28 & 93 \\
\hline C.ATTIVS VI, 4 & 2 & 0 & 4 & 6 \\
\hline C.ATTIVS VI, 5 & & 7 & 3 & 20 \\
\hline C.ATTIVS VI, 6 & 9 & 32 & 19 & 60 \\
\hline C.ATTIVS VI, 8 & 12 & 0 & 3 & 15 \\
\hline C.ATTIVS VI, 9 & 23 & 2 & 7 & 32 \\
\hline C.ATTIVS VI, 10 & 5 & 16 & 6 & 27 \\
\hline CALAMVS IX, 1 & 0 & 0 & 1 & 1 \\
\hline FAVSTVS XII, 1 & 4 & 0 & 2 & 6 \\
\hline FRONTO XVI, 1 & 2 & 1 & 1 & 4 \\
\hline FRONTO XVI, 2 & 56 & 0 & 2 & 58 \\
\hline FRONTO XVI, 4 & 44 & 0 & 2 & 46 \\
\hline FRONTO XVI, 5 & 2 & 0 & 1 & 3 \\
\hline FRONTO XVI, 6 & 1 & 0 & 0 & 1 \\
\hline GAMVS XVII, 1 & 1 & 0 & 3 & 4 \\
\hline GAMVS XVIII, 2 & 2 & 0 & 0 & 2 \\
\hline TM.FORTVNATVS XXIII, 1 & 0 & 113 & 2 & 115 \\
\hline T.M.FORTVNATVS XXIII, 2 & 0 & 5 & 0 & 5 \\
\hline RASINIVS XXVII, 5-6 & 141 & 5 & 10 & 156 \\
\hline RASINIVS XXVII, 7 & 0 & 0 & 1 & 1 \\
\hline RVSTICVS XXVIII, 1 & 11 & 49 & 15 & 75 \\
\hline RVSTICVS XXVIII, 2 & 0 & 3 & 0 & 3 \\
\hline C.SENTIVS XXIX, 2 & 141 & 1 & 18 & 160 \\
\hline C.SENTIVS XXIX, 3 & 1 & 15 & 1 & 17 \\
\hline C.SENTIVS XXIX, 4 & 39 & 1 & 9 & 49 \\
\hline C.SENTIVS XXIX, 5 & 1 & 0 & 0 & 1 \\
\hline C.SENTIVS XXIX, 6 & 9 & 0 & 1 & 10 \\
\hline C.SENTIVS XXIX, 7 & 3 & 0 & 1 & 4 \\
\hline C.SENTIVS XXIX, 10 & 0 & 0 & 7 & 7 \\
\hline STABILIO $x x x$ & 1 & 0 & 0 & 1 \\
\hline L.THYRSVS XXXIII, 3 & 2 & 0 & 0 & 2 \\
\hline L.THYRSVS XXXIII, 4 & 31 & 2 & 1 & 34 \\
\hline L.THYRSVS XXXIII, 5 & 6 & 5 & 0 & 11 \\
\hline L.THYRSVS XXXIII, 6 & 1 & 0 & 0 & 1 \\
\hline L.THYRSVS XXXIII, 7 & 1 & 0 & 0 & 1 \\
\hline L.THYRSVS XXXIII, 8 & 67 & 4 & 3 & 74 \\
\hline L.THYRSVS XXXIII, 10 & 8 & 0 & 0 & 8 \\
\hline L.THYRSVS XXXIII, 12 & 1 & 0 & 0 & 1 \\
\hline L.THYRSVS XXXIII, 13 & 5 & 0 & 1 & 6 \\
\hline L.THYRSVS XXXIII, 19 & 0 & 3 & 0 & 3 \\
\hline TIGRANVS XXXIV, 1 & 56 & 5 & 41 & 102 \\
\hline TIGRANVS XXXIV, 5 & 5 & 0 & 1 & 6 \\
\hline TVLLLIVS XXXV, 1 & 23 & 0 & 5 & 28 \\
\hline SEX.VARIVS XXXVIII, 1 & 0 & 1 & 0 & 1 \\
\hline VLON XLL, 1 & 40 & 0 & 14 & 54 \\
\hline VLON XLL, 4 & 1 & 0 & 0 & 1 \\
\hline anépigraphes & 0 & 2 & 0 & 2 \\
\hline total & 862 & 310 & 252 & 1424 \\
\hline
\end{tabular}

sistes ou transporteurs) qui assuraient en aval l'acheminement des productions? Des " marchands de poterie de terre " et des negotatiores artis cretariae sont connus notamment à Lyon grâce à un certain nombre d'inscriptions funéraires. Bien que ces inscriptions semblent plus tardives que l'époque qui nous intéresse, elles montrent que dans l'Antiquité la diffusion des céramiques se faisait dans un cadre donné et était assurée par des gens dont c'était le métier. Si l'on reprend ce schéma pour l'époque augustéenne, et en particulier pour la diffusion des sigillées lyonnaises, on peut penser que les petits ateliers ne jouaient pas un rôle direct ou important en la matière; les ateliers de plus grande envergure auraient eu, comme les plus modestes, tout intérêt à s'associer à des professionnels (marchands, grossistes et/ou transporteurs) dont le travail était d'adapter l'offre à la demande et d'assurer l'approvisionnement des marchandises. Une telle situation suppose cependant l'existence sous Auguste d'un système " commercial " très étroitement organisé dont la réalité n'est pas formellement attestée à ce jour par des inscriptions funéraires, par des textes ou par toute autre découverte archéologique.

\section{Étude des calibres par marque, ensembles I et II}

L'étude des calibres porte sur l'ensemble des bols signés des ensembles I et II, parmi lesquels seules les séries les plus abondantes ont été prises en compte : Alpvs, Attivs, Fronto, T. M. Fortvnatvs, Rasinivs, Rvsticvs, Sentivs, Thyrsvs, Tigranvs, Vlon.

Les diamètres sont compris entre 3 et $7 \mathrm{~cm}$; quelques exemplaires offrent des diamètres supérieurs à $7 \mathrm{~cm}$. Les histogrammes (fig. 55 à 64 ) montrent que les potiers qui signaient exclusivement des bols, soit Alpvs, Fronto et $\mathrm{T}$. M. Fortvnatvs, produisaient toujours des petits vases. $\grave{A}$ l'inverse, les potiers qui fabriquaient toutes les pièces des différents services (Attivs, Sentivs, Thyrsvs) travaillaient sur une gamme de calibres beaucoup plus étendue.
Fig. 54. Répartition générale des marques par service.

\section{LA CÉRAMIQUE À PAROIS FINES LISSES} (fig. 65 à 72 ; pl. 32 à 40)

Les vases à parois fines des dix contextes ont été inventoriés et étudiés selon les mêmes méthodes de comptage que celles employées pour les céramiques sigillées. Cette catégorie comprend une majorité de vases à pâte siliceuse aux côtés desquels figure un lot de vases à pâte calcaire 


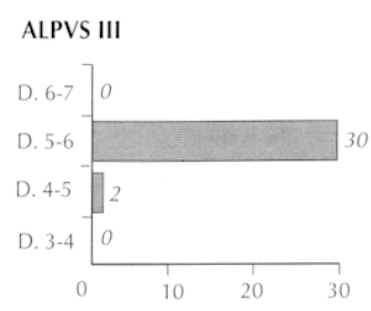

Fig. 55. ALPVS, diamètres des fonds.

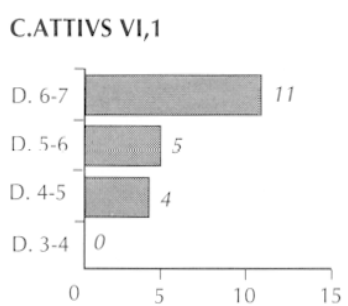

C.ATTIVS VI, 3

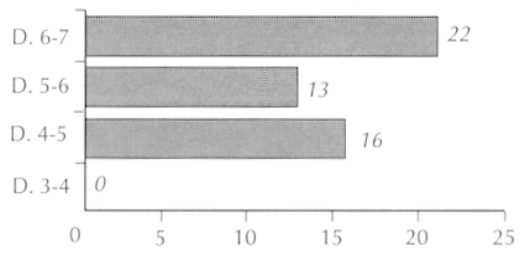

C.ATTIVS VI,8-9-10

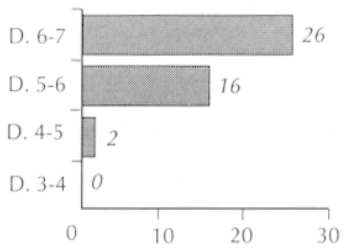

Fig. 56. ATTIVS, diamètres des fonds.

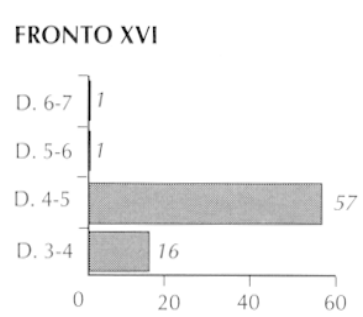

Fig. 57. FRONTO, diamètres des fonds.

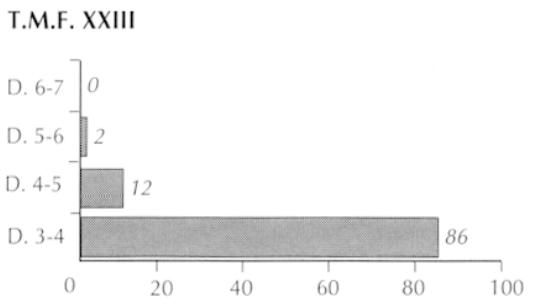

Fig. 58. FORTVNATVS, diamètres des fonds.

RASINIVS XXVII, 5-6

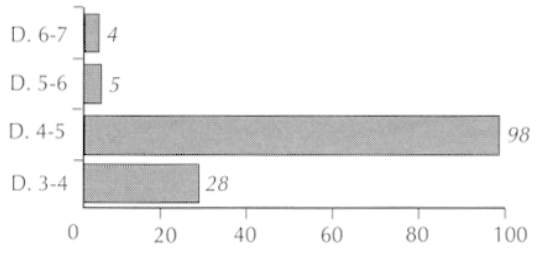

Fig. 59. RASINIVS, diamètres des fonds.

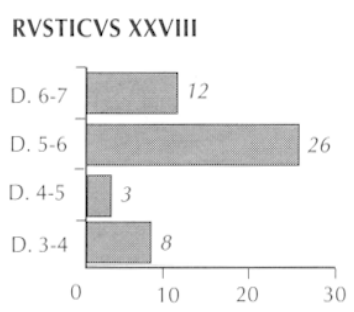

Fig. 60. RVSTICVS, diamètres des fonds.

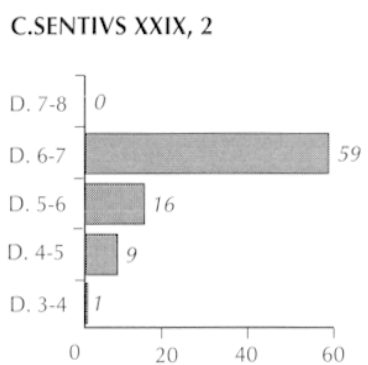

C.SENTIVS XXIX, 2-3-4-6

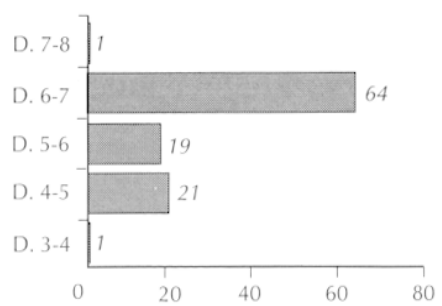

Fig. 61. SENTIVS, diamètres des fonds. 


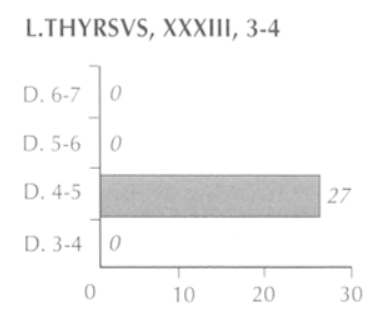

L.THYRSVS, XXXIII, 8

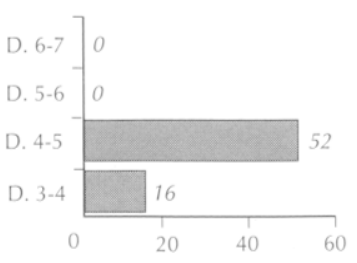

L.THYRSVS, XXXIII, 3-4-8

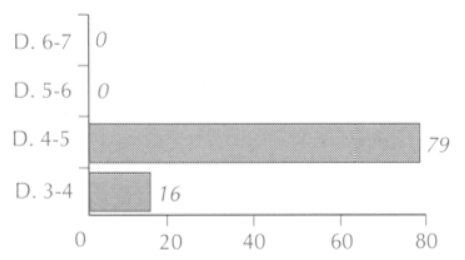

Fig. 62. THYRSVS, diamètres des fonds.

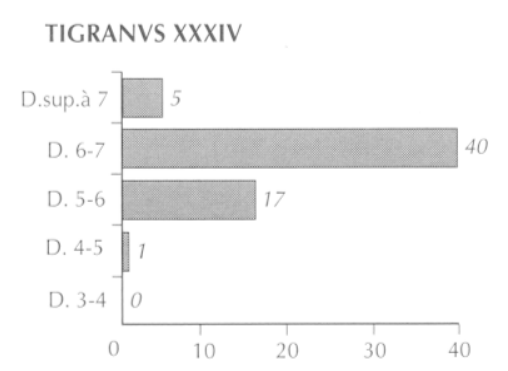

Fig. 63. TIGRANVS, diamètres des fonds.

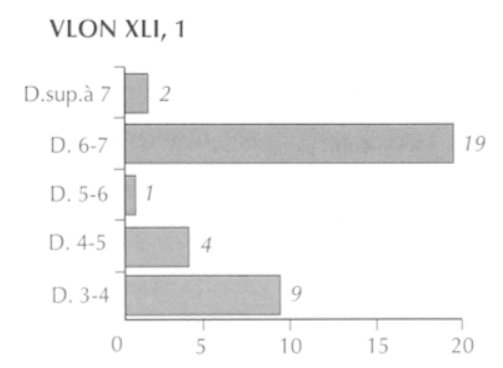

Fig. 64. VLON, diamètres des fonds. qui, bien que restreint, présente un intérêt particulier du fait même de sa rareté à l'époque augustéenne.

\section{LES DONNÉES NUMÉRIQUES (fig. 65 et 66)}

La répartition du matériel par contexte (fig. 65), puis par ensemble (fig. 66), montre tout d'abord d'importants déséquilibres numériques : les contextes de l'ensemble I rassemblent en effet 58561 fragments de bords et de fonds et ceux de l'ensemble II, pourtant très riches en sigillée comme on a pu le voir, n'en comprennent que 1047.

\section{ÉTUDE DES PRODUCTIONS}

\section{LES DONNÉES NUMÉRIQUES}

L'étude de ce matériel extrêmement fragmenté a fait ressortir de nouveaux problèmes qui concernent la méthode de comptage. Prenons pour exemple le mobilier de l'ensemble I : le tableau des fréquences respectives des différents groupes de bords (41 937 fragments) (fig. 67) met en évidence une très nette prédominance des gobelets-tonneaux (groupe $1=58 \%$ ) mais les chiffres donnés pour les gobelets tronconiques (groupe 2 $=3,5 \%$ ) et les gobelets cylindriques (groupe $3=0,1 \%$ ) restent imprécis : la dimension des tessons fait qu'un nombre considérable de fragments se retrouvent, par défaut, dans un groupe d'indéterminés. On a donc tenté de voir si le même travail portant cette fois sur les fonds pouvait aider à préciser davantage. Ce deuxième comptage permet en effet de déterminer plus exactement les fréquences des gobelets tronconiques et cylindriques ainsi que des bols hémisphériques, mais il fausse totalement l'image numérique des gobelets-tonneaux dont les fonds sont peu fragmentés et par conséquent beaucoup moins nombreux. Les deux types de comptage sont donc présentés dans le tableau qui suit, et, en dépit de ces problèmes de méthode qu'il semblait utile d'exposer en préambule, on voit que le mobilier pris globalement ou non est suffisamment abondant pour se prêter à une étude statistique (fig. 67).

Les groupes 1 à 4 correspondent aux quatre types majoritaires reconnus sur l'atelier (Lasfargues, Vertet, 1970). Nous avons classé dans les groupe 5 et 6 les bords droits et un certain nombre de fonds pourant se rattacher aux groupes 2, 3 ou 4, mais dont l'état de fragmentation interdit toute identification précise. Le groupe 7 (divers) 
Fig. 65. Répartition des vases à parois fines dans les dix contextes.

\begin{tabular}{|l|c|c|}
\cline { 2 - 3 } \multicolumn{1}{c|}{} & ensemble I & ensemble II \\
\hline bords & 41937 & 660 \\
\hline fonds & 16624 & 387 \\
\hline total & 58561 & 1047 \\
\hline
\end{tabular}

Fig. 66. Répartition des vases à parois fines par ensemble.

\begin{tabular}{|c|c|c|c|}
\hline GROUPES & & E.1 & E.2 \\
\hline gobelets-tonnea & & 33071 & 136 \\
\hline & bords & 24239 & 102 \\
\hline & fonds & 8832 & 34 \\
\hline gobelets troncor & ques & 3029 & 40 \\
\hline & bords & 1426 & 15 \\
\hline & fonds & 1603 & 25 \\
\hline gobelets cylindr & ues & 996 & 7 \\
\hline & bords & 65 & 0 \\
\hline & fonds & 931 & 7 \\
\hline bols hémisphéric & & 7888 & 846 \\
\hline & bords & 3014 & 531 \\
\hline & fonds & 4874 & 315 \\
\hline bords droits inde & erminés & 12288 & 0 \\
\hline fonds indétermir & & 120 & 0 \\
\hline divers & & 474 & 14 \\
\hline & bords & 434 & 9 \\
\hline & fonds & 40 & 5 \\
\hline pâtes calcaires & & 695 & 4 \\
\hline & bords & 471 & 3 \\
\hline & fonds & 224 & 1 \\
\hline total & & 58561 & 1047 \\
\hline
\end{tabular}

Fig. 67. Répartition des sept groupes de vases à parois fines dans les deux ensembles.

comprend les types minoritaires, parfois inédits, ne présentant aucun point commun avec les précédents et le groupe 8 des vases à pâte calcaire déterminant euxmêmes différents types.

\section{ENSEMBLE I : ÉTUDE TYPOLOGIQUE}

(fig. 68 à $72 ;$ pl. 32 à 38 )

Le mobilier de l'ensemble I, particulièrement abondant, présente une image typologique très homogène puisque $98 \%$ des vases appartiennent aux quatre principaux types produits sur l'atelier (groupes 1 à 5) (fig. 72). L'ensemble des vases offre un aspect extrêmement soigné témoignant d'une grandc finesse d'exécution : les gobelets tronconiques et cylindriques ainsi que les bols hémisphériques ont de façon générale des surfaces parfaitement lisses et les derniers portent parfois un léger guillochis sur la partie supérieure de la panse. À l'inverse, les gobelets-tonneaux offrent assez souvent des surfaces granuleuses au toucher. Ces quatre types sont fort bien connus à l'époque augustéenne sur le limes comme sur les principaux sites de consommation.

Groupe 1 : gobelets-tonneaux (fig. 68 ; pl. 32 et 33), constitue, il est vrai, l'essentiel de cet ensemble avec $58 \%$ des bords identifiés : l'examen détaillé de ces profils révèle un chapelet de variantes fines : les lèvres peuvent être épaissies " en amande ", à face interne ou externe légèrement concaves, et forment des embouchures plus ou moins évasées. L'étude des diamètres d'ouverture effectuée sur un échantillon de cent exemplaires montre que les dimensions extrêmes (inférieures à $5-6 \mathrm{~cm}$ ou supérieures à $12 \mathrm{~cm}$ ) restent très rares : la majeure partie des bords (75 ex.) sont en effet compris entre 7 et $9 \mathrm{~cm}$ (fig. 69).

Groupe 2 : gobelets tronconiques (pl. 34, 1-6), bien que comparativement très peu représenté avec 1426 fragments de bords $(3,4 \%)$ et 1603 fragments de fonds $(9,5 \%)$, se distingue par une très grande uniformité morphologique comme le montrent les quatre exemplaires complets.

Groupe 3: gobelets cylindriques (pl. 34, 7-15), nettement minoritaire, que l'on prenne en compte les bords $(0,1 \%)$ ou les fonds $(5,6 \%)$ correspond en sigillée au type Haltern 16 également produit sur l'atelier comme on a pu le voir. Malheureusement, aucun profil complet n'a 
pu être reconstitué : les parois sont rectilignes et adoptent parfois un profil légèrement érasé; le fond, de forme concave, repose sur un pied finement mouluré.

Croupe 4 : bols hémisphériques (fig. 70 et pl. 35) qui rassemble ici $7 \%$ des bords et $29 \%$ des fonds comprend une quinzaine de formes complètes parmi lesquelles on note seulement quelques variantes sensibles : elles peurent concerner d'une part les rapports dimensionnels (voir le rapport diamètre d'ouverture/hauteur du bol 14), d'autre part l'orientation des parois (voir le profil rentrant 1 et, à l'inverse le profil éversé du 2). Quelques exemplaires possèdent un décor guilloché plus ou moins maladroit et souvent presque effacé (1亏). Les surfaces sont en majorité lisses et uniformes, mais on note parfois des traitements de surface comparables à ceux observés sur une partie des gobelets-tonneaux.

Les rases n'appartenant pas aux groupes 1 à jo sont au nombre de neuf cent cinq et correspondent à $2 \%$ du total des bords. Quatre cent trente-quatre d'entre eux possèdent des pâtes siliceuses (pl. 36 et 37) et quatre cent soixante et onze des pâtes calcaires (pl. 38).

\section{Les pâtes siliceuses}

Cent vingt-cinq vases correspondent d'une part à des gobelets ansés (pl. 36, 1-11), d'autre part à des bols ou coupes à lèrre éversée ne se rattachant pas à des tỵpes bien définis (pl. 36, 12-24); dans ce lot, une dizaine de bords arrondis en bourrelet pourraient appartenir à des petits pots ovoïdes (pl. 36, 18-22).

Trois cent neuf bords déterminent ensuite un type original de gobelets à bord strié, comprenant lui-même deux séries distinctes : les stries se situent dans la plupart des cas sur la partie supérieure du rebord (pl. 37, 1-15) mais parfois aussi à la liaison bord/panse (pl. 37, 16-19). Chacune des deux séries ne constitue pas cependant un type figé : les profils peuvent adopter une forme cylindrique ou tronconique, la place et la succession des stries sur le bord ou à mi-panse sont rarement identiques d'un exemplaire à l'autre. Il s'agit là d'un tỵpe inédit à Lỵon (Verbe-Incarné, rue des Farges) et apparemment inconnu sur le limes comme ailleurs.

\section{Les pâtes calcaires}

Parmi quatre cent soixante et onze bords, trois cent deux sont de fidèles " imitations " des gobelets tronco-

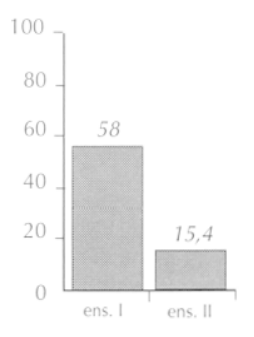

Fig. 68. Cobelets-tomneaux, pourcentages respectifs des bords dans les deux ensembles.

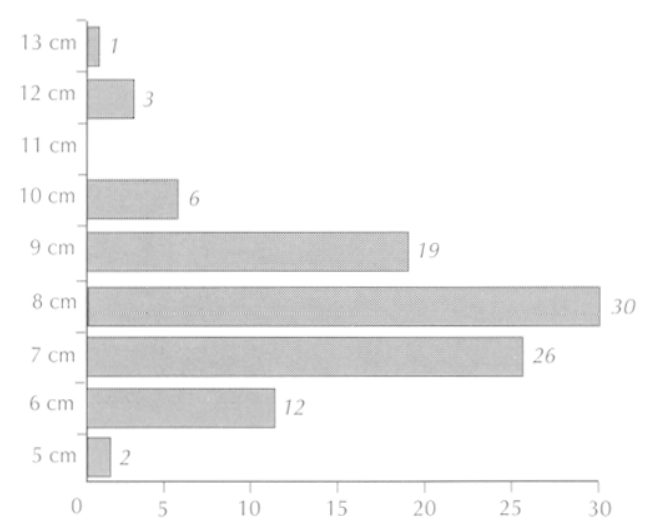

Fig. 69. Ensemble I, gobelets-tonneaux, variation des diametres des bords sur cent exemplaires.

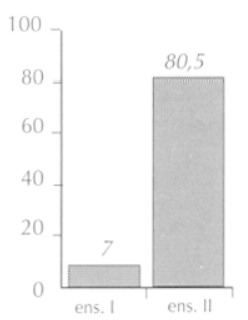

Fig. 70. Bols hémisphériques, pourcentages respectifs des bords de la totalite des vases dans les deux ensembles.

niques et cylindriques, des gobelets à bord strié ainsi que des bols hémisphériques (pl. 38, 1-5) ; parmi les fonds à pâte calcaire se trouvent d'ailleurs plusieurs exemples prouvant qu'on peut réellement parler de "copies conformes " aux originaux (pl. 38, 12-19). Les cent soixante-neuf derniers bords forment en revanche un type original de coupe haute ou gobelet à lèrre éversée pour lequel on ne trouve pas de correspondance dans les typologies des parois fines augustéennes (pl. 38, 6-7, 910); un dernier type, également inédit, est figuré par une coupe évasée à lèrre en bourrelet et anses latérales (pl. 38, 11).

Cet échantillon de parois fines reste unique à Lyon : les sites du Verbe-Incarné et de la rue des Farges n'ont en 
effet pas livré plus que les autres un seul tesson ou vase présentant les mêmes caractéristiques de pâte et d'aspect.

ENSEMBLE II : ÉTUDE TYPOLOGIQUE (fig. $68,69,71$ et 72 ; pl. 39 et 40$)$

Le second ensemble de vases à parois fines, bien que très faible numériquement, montre des différences notables tant sur le plan statistique que typologique (fig. 72).

La répartition typologique se modifie nettement : on observe une chute spectaculaire des gobelets-tonneaux qui passent de $58 \%$ à $15 \%$; cette chute semble avoir pour corollaire une très forte augmentation des bols hémisphériques qui, de $7 \%$ des bords et $29 \%$ des fonds dans l'ensemble I, vont ici jusqu'à dépasser $80 \%$ des bords et $82 \%$ des fonds. On note d'ailleurs certains changements dans l'aspect des surfaces et la morphologie des bols : les lèvres

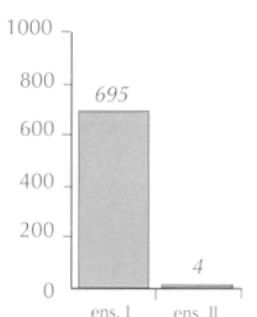

Fig. 71. Pourcentages respectifs des vases à pâte calcaire dans les deux ensembles.

ensemble I

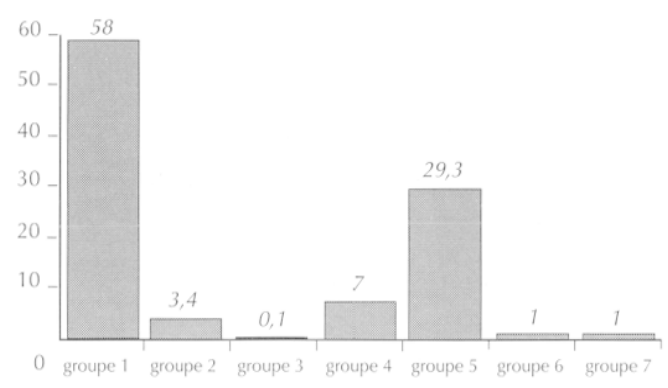

ensemble II

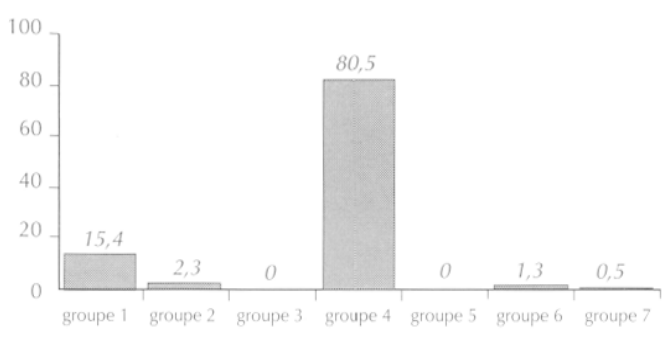

Fig. 72. Représentalion respective des sept groupes de vases à parois fines dans les deux ensembles. deviennent de plus en plus rentrantes et les parois, en très grande majorité, "granitées " ou granuleuses au toucher. C'est dans ce groupe qu'apparaissent aussi trois bols granités à pâte et surface grises ou "bols gris " qui correspondent à une série annexe des bols hémisphériques classiques, sans doute d'origine italique (pl. 39, 13-14). Cette série est attestée à Lyon dès le début de notre ère sur le site du Verbe-Incarné (Genin, 1993); on la trouve au Magdalensberg dans des contextes compris entre 10 avant J.-C. et 25 après J.-C. (Schindler, Kaudelka, 1975) ainsi qu'à Ravenne dans la première moitié du $\mathrm{I}^{\mathrm{cr}} \mathrm{s}$. après J.-C. (Maioli, 1972).

Les gobelets à bord strié et le groupe des vases à pâte calcaire deviennent rarissimes dans l'ensemble II. En revanche, il faut mentionner quatre fragments ( 3 bords, 1 fond) déterminant des pots ovoïdes à dépressions (pl. 40, 4-7). Les deux modules représentés offrent le même type de lèvre linéaire légèrement éversée; les surfaces sont noires, granitées et possèdent plutôt l'aspect de vases ratés que de vases à parois fines proprement dits. Il semble possible d'assimiler ces exemplaires au type Haltern 44, type rare et de ce fait peu connu (Loeschcke, 1909, pl. 26, 4).

Les changements que révèlent les céramiques à parois fines de l'ensemble II constituent autant d'indices chronologiques venant conforter l'hypothèse de deux phases de production.

M. G.

\section{LES GOBELETS D'ACO}

Les gobelets d'Aco qui constituent une part importante du mobilier des fosses "Gobelet I et II " ont été regroupés dès l'origine et forment donc un seul lot. Les gobelets issus des contextes du sondage de 1975 forment un lot beaucoup moins important mais qui apporte quelques données complémentaires.

Tous ces gobelets appartiennent à la même phase de production (ensemble I) et on a pu réaliser des collages entre le mobilier de 1966 et celui de 1975. Par ailleurs, la quantité de gobelets dans chaque contexte de la fouille de 1975 est trop faible pour justifier une étude par contexte. Pour ces raisons, il n'a pas semblé utile de présenter séparément les gobelets de 1966 de ceux de 1975 et l'étude a pris en compte la totalité du matériel qui sera présenté globalement. 
Ce matériel a fait l'objet d'un comptage sur le modèle du mobilier sigillé (fig. 73).

\begin{tabular}{|l|c|c|c|}
\hline & Gobelet I et II & Muette 75 & total \\
\hline nombre de fonds & 1630 & 130 & 1760 \\
\hline nombre de bords & 715 & 68 & 783 \\
\hline
\end{tabular}

Fig. 73. Répartition des gobelets d'Aco, fonds et bords.

\section{CARACTÉRISTIQUES TECHNIQUES ET TYPOLOGIE}

Tous les gobelets d'Aco ont été façonnés en pâte siliceuse. On note cependant une diversité dans la texture et le coloris des argiles utilisées. Celles-ci varient du beige rosé au brun rouge foncé. Elles comportent souvent un fin dégraissant micacé. Quelques exemplaires surcuits présentent une teinte gris foncé. Aucun gobelet n'a reçu de revêtement argileux, notamment de vernis rouge de type sigillée. En revanche, deux fragments présentent des traces d'une couverte blanchâtre, qui ne ressemble ni à un engobe ni à un dépôt calcaire (cf. pl. 67, 24). L'analyse a confirmé qu'il s'agissait d'une glaçure au plomb, insuffisamment cuite, comme le suggérait la comparaison avec certains gobelets de Saint-Romain-en-Gal. Cet indice laisse supposer que l'atelier de la Muette a produit également quelques gobelets à glaçure plombifère, comme ceux de Loyasse et de Saint-Romain-en-Gal, bien qu'aucune autre trace de cette production n'ait été découverte.

Seize exemplaires dont le profil est complet permettent de distinguer plusieurs gabarits dans les gobelets, qui se répartissent en deux groupes :

- les formes hautes (pl. 41, 1-11) : leur taille varie de 115 à $95 \mathrm{~mm}$ et leur diamètre supérieur de 61 à $81 \mathrm{~mm}$. La majorité des exemplaires se place vers $10 \mathrm{~cm}$ de haut. Le rapport $\mathrm{H} / \mathrm{D}$ se situant à 1,5 .

- les formes basses (pl. 41, 12-16) : moins nombreuses que les formes hautes, elles regroupent des gobelets dont la hauteur varie de 63 à $94 \mathrm{~mm}$ et le diamètre de 72 à $91 \mathrm{~mm}$. Le rapport H/D se situant entre 0,8 et 1 .

Comme sur tous les gobelets d'Aco, le col tourné hors du moule se termine par une lèvre formant un léger bourrelet. Les fonds sont tournassés de manière systématique, le tournassage s'accompagne de l'aménagement d'une mouluration sur le pied, à la base du décor. Il peut s'agir d'une simple gorge ou d'une moulure double ou triple (fig. 74), quelquefois celle-ci mord sur le décor. Seuls quelques rares gobelets (notamment un gobelet de T. Cavivs) ne présentent pas de tournassage (cf. pl. 65, 2). Ce traitement particulier du pied semble caractéristique des productions de la Muette et ne se rencontre ni dans l'atelier de Loyasse, ni dans celui de Saint-Romain-en-Gal, en dépit de la similitude des décors (Desbat, 1985). Le col du gobelet subit lui-même un tournassage qui peut mordre sur le décor de la frise, comme le montrent plusieurs exemples. Le procédé de façonnage explique que des vases issus du même moule puissent avoir une taille différente (cf. par exemple pl. 65).

\section{LES SIGNATURES, MARQUES INTRADÉCORATIVES}

Quatre cent trente-huit marques ont été dénombrées sur les gobelets, auxquelles il faut ajouter deux marques sur moule. Elles livrent les signatures suivantes (fig. 75) :

- ACO : 7 ex.

- HILARVS : 18 ex.

- HILARVS ACO : 114 ex.

- CHRYSIPPVS : $251 \mathrm{ex}$.

- PHILARCVRVS : 33 ex.

- T. CAVIVS : 8 ex.

- PHILARCVRVS T. CAVIVS : 2 ex.

- PHILOCRATES : 5 ex.

- FIDELIS : 2 ex.

\section{ACO}

La signature Aco, seule, ne figure qu'à sept exemplaires avec deux graphismes (fig. 76 et pl. 66).

1.1. ACO. Inscrit dans la frise. Lettres de 4 et $4,5 \mathrm{~mm}$ de haut (2 exemplaires). (Décor 1, pl. 66).

1.2. ACO. La signature s'inscrit dans le décor de picots. Hauteur des lettres $4,5 \mathrm{~mm}$, (5 exemplaires). (Décor 2, pl. 66).

\section{HILARVS (fig. 77 et pl. 66)}

2.1. HILARVS. La signature est formée de lettres imprimées séparément, de hauteur variable, sans motif d'encadrement; $\mathrm{L}: 3 \mathrm{~mm}, \mathrm{H}: 4 \mathrm{~mm}, \mathrm{~S}: 5 \mathrm{~mm}$. (Décors 1 et 2 , pl. 66).

2.2. HIL (A) RVS. Lettres imprimées séparément, de hauteur variable : $\mathrm{H}, \mathrm{I}, \mathrm{L}: 3 \mathrm{~mm}$; $\mathrm{S}: 4 \mathrm{~mm}$; V formé de deux barres; la signature est encadrée par deux masques. (Décor 3, pl. 66). 


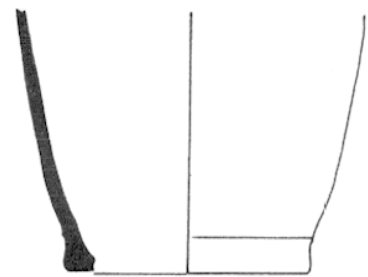

1

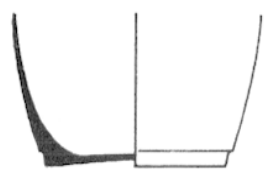

5

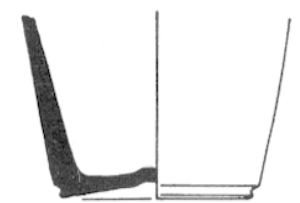

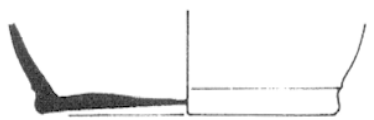

2

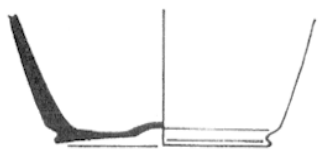

6

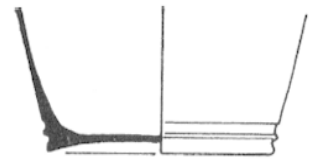

10

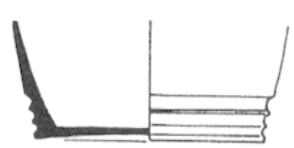

13

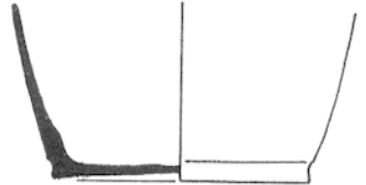

3

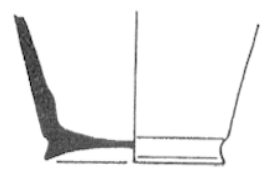

7

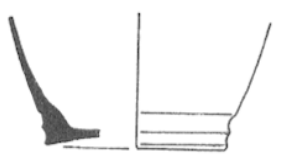

11

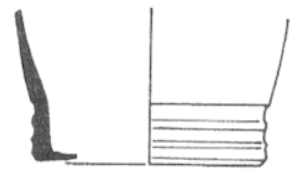

14

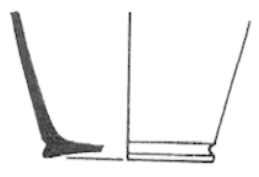

4
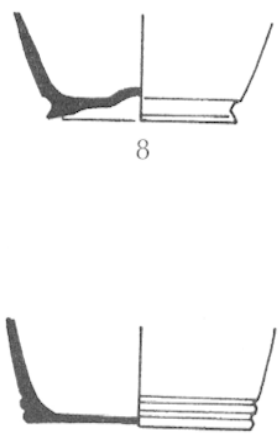

12

Fig. 74. Différents exemples de mouluration des fonds de gobelets (échelle: 1/2).

\begin{tabular}{|l|r|r|}
\hline \multicolumn{1}{|c|}{ signature } & nombre & \multicolumn{1}{c|}{$\%$} \\
\hline Chrysippvs & 251 & \multicolumn{1}{c|}{57} \\
\hline Aco - Hilarvs-Aco & 139 & 31,5 \\
\hline T. Cavivs & 8 & 1,8 \\
\hline Philarcvrvs & 33 & 7,5 \\
\hline Philarcvrvs Cavivs & 2 & 0,45 \\
\hline Philocrates & 5 & 1,1 \\
\hline Fidelis & 2 & 0,45 \\
\hline total & $\mathbf{4 4 0}$ & \\
\hline
\end{tabular}
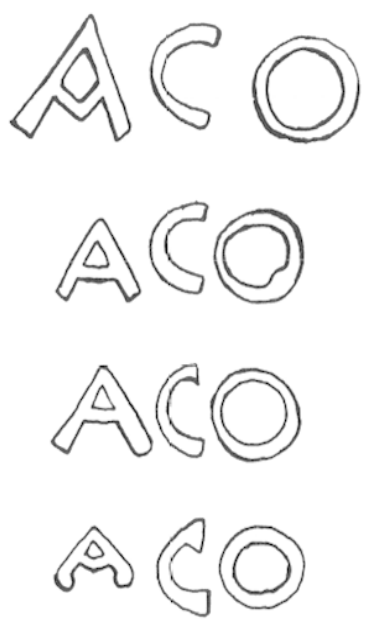

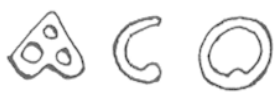

Fig. 75. Répartition des différentes signatures des gobelets d'Aco.

\& $C(9)$

Fig. 76. Différents graphismes du nom d'Aco (échelle: $2 / 1$ ). 


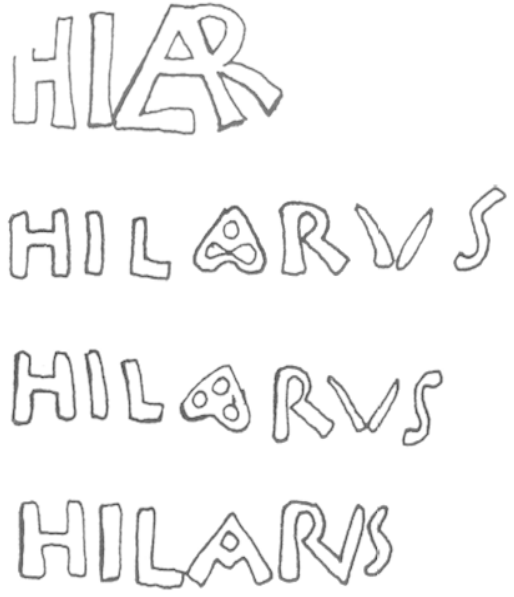

Fig. 77. Differents graphismes d'Hilarus (échelle: 2/1).

2.3. (HILA) RVS entre deux abeilles. (Décor 4, pl. 66).

2.4. (HILAR) VS entre deux motifs de bourgeons verticaux. (Décor 28, pl. 67).

2.5. P/HILARVS. Le $\mathrm{P}$ est situé sous le $\mathrm{H}$ d'Hilarvs. Cette signature qui n'est représentée à la Muette que par quatre exemplaires existe à Saint-Romain-en-Gal. La hauteur des lettres est de 3,5 à $5 \mathrm{~mm}$. Le A est formé avec une barre horizontale et le $\mathrm{R}$ et le $\mathrm{V}$ sont liés. Pas de motif d'encadrement. (Décor 5, pl. 66).

\section{HILARVS ACO}

La signature HILARVS ACO est une des plus fréquentes; elle apparaît sous plusieurs formes et avec plusieurs graphismes.

3.1. HILAR (vs) ACO. Cette signature inédite figure à huit exemplaires; hauteur des lettres $5,5 \mathrm{~mm}$. Elle comporte une ligature LAR. Le A de Aco présente une barre en $\mathrm{V}$, alors que celui de Hilar comporte une barre horizontale. (Décor 6, pl. 66).

C'est sous la forme HILARVS ACO que la signature est la plus fréquente. Elle est formée de lettres imprimées séparément, hautes de 3 à $3,5 \mathrm{~mm}$. Le A est formé d'un triangle avec trois cercles. Ce graphisme, de loin majoritaire, se rencontre le plus souvent encadré de différents poinçons qui permettent de distinguer plusieurs moules; elle peut se rencontrer également sans motif d'encadrement. Les deux noms sont fréquemment séparés par un picot, plus rarement par un point.
3.2. HII ARVS ACO, sans motif d'encadrement un picot entre Hilarvs et Aco. Le O est formé de deux $C$ affrontés, le $V$ de deux barres non jointives. (Décors $7,8,9$ et 11 , pl. 66).

3.3. Un fleuron à six pétales entre Hilarvs et Aco. (Décor 14, pl. 66).

3.4. Entre deux fleurons à six pétales, motif de séparation? (Décor 12, pl. 66).

3.5. Entre deux feuilles bilobées, avec un point de séparation. (Décor 16, pl. 67).

3.6. Entre deux feuilles bilobées avec un picot en séparation. (Décors 10, pl. 66 et 15, pl. 67)

3.7. Entre deux abeilles, avec une guirlande sous la signature. (Décor 19, pl. 67).

3.8. Entre deux fleurons à huit pétales, motif de séparation? (Décors 21 et 22, pl. 67).

3.9. Entre deux coquilles Saint-Jacques, picot entre Hilarvs et Aco. (Décor 13, pl. 66).

3.10. Entre deux coquilles Saint-Jacques, pas de séparation entre Hilarvs et Aco. (Décor 20, pl. 67).

3.11. Entre deux feuilles de lierre, le $\mathrm{O}$ est ovale. Le A avec une barre horizontale, hauteur : $4 \mathrm{~mm}$. (Décor 23, pl. 67).

3.12. Entre Minerve et une tête. (Décor 24, pl. 67).

3.13. Entre deux masques, avec guirlande sous la signature, pas de séparation cntrc lcs dcux noms. Le $\Lambda$ est différent des autres signatures. (Décor 25, pl. 67).

3.14. Entre deux masques, sans guirlande sous la signature. Le A est du même type que pour 3.13. (Décor 26, pl. 67).

3.15. Entre deux feuilles de lierre verticales. Un picot entre Hilarvs et Aco (signature du moule). (Décor 29, pl. 67).

3.16. Signature incomplète à un seul exemplaire. (Hil) ARVS ACO, avec ligature RV et picot entre Hilarvs et Aco. On ignore si elle comporte un motif d'encadrement. (Décor 27, pl. 67).

\section{CHRYSIPPVS}

C'est de loin la signature la plus fréquente avec deux cent cinquante exemplaires. Elle est formée comme celle d'Hilarvs Aco de lettres séparées d'une hauteur de 3 à $3,5 \mathrm{~mm}$. Elle se rencontre également associée à différents motifs d'encadrement (pl. 64).

4.1. CHRYSIPPVS entre deux coquilles Saint-Jacques. (Décors 40 et 41, pl. 61,62 et 64). 
4.2. Entre deux feuilles de lierre (?). (Décors 43,46 et 47 , pl. 64).

4.3. Entre deux fleurons à huit pétales. (Décor 42, pl. 64).

4.4. Entre deux fleurons, au-dessus d'une guirlande (Décor 6, pl. 46).

4.5. Entre deux masques, au-dessus d'une guirlande. (Décors 44 et 45, pl. 64).

4.6. CHRY - SIPPVS de part et d'autre d'une Fortune. (Décor 26, pl. 57).

4.7. CHRYSI.- PPVS idem.

4.8. Entre deux dauphins. (Décors 14 et 19, pl. 52 et 55).

4.9. Entre deux vires. (Décor 38, pl. 53 et 61 ).

4.10. Entre deux étoiles (moule). (Décors 9,10 et 11 , pl. 50 et 51 ).

4.11. Entre deux masques. (Décors 33 et 37, pl. 59 et 60 ).

4.12. Entre deux bouquets d'acanthe, au-dessus d'une guirlande. (Décor 25, pl. 56).

4.13. Entre deux aigles, au-dessus d'une guirlande. (Décor 5, pl. 46 et 47).

\section{CHRYSIPPVS (ARS)}

À la différence des signatures précédentes, celle-ci n'est pas formée par l'impression de poinçons séparés mais par le surmoulage d'une estampille complc̀te de Chrysippvs, complété par le mot ARS. À la Muette, la signature incomplète n'est connue qu'en quatre exemplaires, mais elle existe également dans l'atelier de SaintRomain-en-Gal qui a fourni des signatures complètes, toutes du même moule. Le surmoulage au nom de Chrysippvs permet de supposer que cette signature n'émane pas de Chrysippvs mais d'un potier ayant imité le style de Chrysippvs; le "Chrysippvs ars " s'apparentant à "l'arretinum fecit " de certaines sigillées. (Décor 49 , pl. 64).

\section{PHILARCVRVS}

Cette signature formée également de lettres imprimées séparément est la troisième par importance avec trente-trois exemplaires. Elle se rencontre avec plusieurs graphies, toutes incomplètes (fig. 78). Elle est inconnue dans l'atelier de Saint-Romain-en-Gal.

6.1. PHILARCVRV (s). (Décor 1, pl. 68).

6.2. PHILARCV - RVS. (Décor 2, pl. 68).

6.3. PHILA - RCVR - VS. (Décor 3, pl. 68).

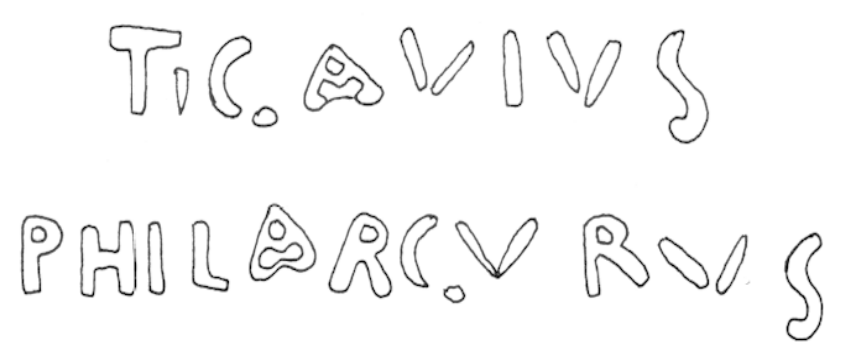

Fig. 78. Signatures de T. Cavivs et de Philarcurvs (échelle : 2/1).

\section{T. CAVIVS}

Seuls huit exemplaires de cette signature ont été recensés. Elle est formée de lettres imprimées séparément, de hauteur variable : A : $3 \mathrm{~mm}$; T, C, S : $5 \mathrm{~mm}$; V : $4 \mathrm{~mm}$. Un point sépare le $\mathrm{T}$ du C. L'extrémité inférieure du C est brisée, et l'on y a vu à tort un point de séparation entre le $\mathrm{C}$ et le $\mathrm{A}$, d'où la lecture T.C.AVIVS proposée à l'origine (fig. 78). Comme les deux précédentes, elle n'existe pas dans l'atelier de Saint-Romain. En revanche, elle est bien représentée à Dangstetten (Fingerlin, 1986). (Décors 1-3, pl. 69).

\section{8. (PHILAR) CVRVS CAVIVS}

On ne connaît que deux exemplaires de cette signature qui associe PHILARCVRVS et CAVIVS, sur une forme basse; hauteur des lettres : $4 \mathrm{~mm}$. (Décor 5 , pl. 65 et 69 ).

\section{PHILOCRAT (ES)}

Connue par six exemplaires incomplets, la signature est formée de lettres imprimées séparément, hauteur 3,5 à $4 \mathrm{~mm}$. Cette signature est également inconnue dans l'atelier de Saint-Romain-en-Gal. (pl. 70).

\section{FIDELIS}

À la différence des autres signatures celle de Fidelis est gravée dans le moule à la pointe, avec des lettres de $5 \mathrm{~mm}$. Un autre fragment permet de lire IT et de restituer la signature sous la forme FIDELIS FECIT. Cette signature se rencontre dans l'atelier de Saint-Romain-en-Gal sous la même forme (pl. 70). 


\section{LES FRISES}

Cinquante frises différentes ont été dénombrées (pl. 42 et 43). La plupart d'entre elles sont associées à des signatures permettant de les attribuer aux différents décorateurs.

\section{LES FRISES SIMPLES}

Frise 1 : motif en forme de double crosse terminé en pointe, orienté vers la droite (hauteur : $3,5 \mathrm{~mm}$ ). Frise d'Hilarss Aco et de Chrysipprs.

Frise 2 : motif semblable mais orienté vers la gauche. (hauteur : $5 \mathrm{~mm}$ ). Frise de Philarcvrvs Cavivs.

Frise 3 : motif de feuilles opposées, orienté vers la gauche (hauteur : $4 \mathrm{~mm}$ ). Frise d'Hilarvs Aco.

Frise 4 : motif de feuilles opposées arrondies, orienté vers la gauche (hauteur: $6 \mathrm{~mm}$ ). Frise de T. Cavivs.

Frise 5: motif de feuilles de lierre, orienté vers la gauche (longueur $6 \mathrm{~mm}$ ). Aucunc signature n'est associéc à cette frise dans l'atelier de la Muctte, mais on la trouve sur les gobelets de T. Cavivs à Dangstetten (Fingerlin, 1986, 397, 19).

Frise 6: motif de feuilles opposées lancéolées et nerrurées, orienté vers la droite (longueur $5,5 \mathrm{~mm}$ ). Frise de Chrysipprs.

Frise 7 : motif semblable mais avec un pétiole (longueur : $7 \mathrm{~mm}$ ). Frise de Chrysippvs.

Frise 8: motif semblable, plus petit (longueur 5 $\mathrm{mm}$ ) obtcnu par surmoulage du motif précédent? Frise d'Hilarvs.

Frise 9: motif de feuilles bilobées avec un pétiole, orienté vers la droite (longueur $6 \mathrm{~mm}$ ). Frise d'Hilarrs.

Frise 10: motif de feuilles trilobées, orienté vers la gauche (hauteur : $6,5 \mathrm{~mm}$ ). Frise de Philarcros et Chrysipprs.

Frise 11: motif semblable orienté vers la droite (hauteur : $6,5 \mathrm{~mm})$. Frise de Chrysipprs.
Frise 12: motif de petites feuilles de lierre, orienté vers la droite (longueur $4 \mathrm{~mm}$ ). Frise d'Hilars's.

Frise 13: motif de feuilles nervurées, à trois pointes, tourné vers la droite (hauteur : $6 \mathrm{~mm}$ ). Frise de Chrysipprs et T. Cavivs.

Frise 14 : motif semblable tourné vers la gauche (hauteur : $5,5 \mathrm{~mm}$ ). Frise d'Hilarus Aco et de Chrysipprs.

Frise 15: motif de fleurons à six pétales nervurés (hauteur : 4,5 mm). Frise d'Hilars et de Philocrates.

Frise 16 : motif de fleurons à six pétales arrondis (hauteur : $5 \mathrm{~mm}$ ). Frisc non attribuée.

Frise 17: motif de fleurons à six pétales nervurés avec le coeur circulaire, creux (hauteur : $5,5 \mathrm{~mm}$ ). Frise de Chrysipprs.

Frise 18: motif de feuilles trilobées nervurées, orienté vers la droite (longueur $6 \mathrm{~mm}$ ). Frise de Chrysipprs.

Frise 19: motif de feuilles opposées, lancéolées, orienté vers la gauche (hauteur: $4 \mathrm{~mm}$ ). Frise de Chrysipprs.

Frise 20: motif de feuilles trilobées avec deux bourgeons latéraux et un pétiole (longueur $6 \mathrm{~mm}$ ), orienté vers la droite. Frise de Chrysippus.

Frise 21 : motif semblable mais sans pétiole (longueur $5 \mathrm{~mm}$ ). Frise de Chrysipprs et Hilarvs Aco.

Frise 22: motif de feuilles à deux folioles nervurées et avec deux bourgeons à la base, orienté vers la droite (longueur $7 \mathrm{~mm}$ ). Frise de (hrysipprs.

Frise 23: motif semblable mais avec un pédoncule central (longueur $7 \mathrm{~mm}$ ). Frise d'Hilarrs Aco.

Frise 24: motif semblable mais avec les folioles et les bourgeons plus petits (longueur $6 \mathrm{~mm}$ ). Frise d'Hilars Aco.

Frise 25: motif de feuilles à deux folioles nervurées et pédoncule central, orienté vers la droite (longueur $6 \mathrm{~mm}$ ). Frise du moule d'Hilarrs Aco. 
Frise 26: motif proche des précédents mais avec folioles plus larges et pédoncule strié (longueur $7 \mathrm{~mm}$ ), orienté vers la droite. Frise de Chrysippvs.

Frise 27 : mulif de petites feuilles dentelées (vigne $\vdots$ ), orienté vers la droite (longueur $7 \mathrm{~mm}$ ). Frise de Chrysippvs.

Frise 28: motif de feuilles opposées avec deux bourgeons, orienté vers la droite (longueur $6 \mathrm{~mm}$ ). Frise de Chrysippus.

Frise 29: motif semblable, plus petit (longueur $5 \mathrm{~mm}$ ). Frise de Chrysippvs, Hilarvs et T. Cavivs.

Frise 30: motif semblable, empâté. Frise de Fidelis.

Frise 31 : motif de bourgeons floraux stylisés, orienté vers la gauche. Frise d'Hilarvs Aco.

Frise 32 : motif d'insectes, scarabées? Frise de Chrysippvs? d'Hilarvs Aco?

Frise 33: motif de feuilles de lierre encadrées par deux points. Frise de Chrysipprs.

Frise 34 : motif semblable, orienté vers la gauche. Frise de Philarcvrvs.

Frise 35 : motif indéterminé encadré par deux points. Frise d'Aco.

Frise 36: motif indéterminé alterné avec une pastille. Frise non attribuée.

\section{LES FRISES DOUBLES}

Plusieurs frises sont formées de deux rangées de motifs superposés.

Frise 37: en haut motif à feuilles opposées, orienté vers la gauche; en bas, motif de fleurons à huit pétales. Frise de Chrysippvs.

lirise 38 : en haut motif de fleurons à six pétales; en bas motif de feuilles opposées, orienté vers la droite. Frise d'Hilarvs Aco et de Chrysippus.
Frise 39: en haut motif de feuilles opposées, orienté à droite; en bas idem mais plus petit. Frise d'Hilarvs Aco.

Frise 40: en haut motif de feuilles opposées, orienté à gauche; en bas motif de petites feuilles de lierre. Frise d'Hilarvs Aco.

Frise 41: en haut, motif de feuilles opposées, orienté vers la gauche; en bas motif de petites feuilles trilobées, orienté à gauche. Frise d'Aco.

Frise 42 : en haut motif de feuilles nervurées opposées, orienté à droite; en bas motif de fleurons à huit pétales. Frise de Chrysippvs.

Frise 43: en haut motif de feuilles nervurées opposées, orienté à gauche; en bas motif de fleurons à huit pétales. Frise de Chrysipprs.

Frise 44 : en haut motif de fleurons à six pétales nervurés; en bas motif de feuilles trilobées, orienté à droite. Frise d'Hilarvs Aco.

Frise 45 : en haut motif de feuilles opposées, orienté à gauche. En bas motif de points. Frise de Philarcurvs.

Frise 46 : en haut motif de feuilles opposées avec deux bourgeons latéraux, orienté à gauche; en bas, motif de feuilles opposées nervurées, orienté à gauche. Frise d'Hilarvs.

Frise 47 : en haut motif de feuilles d'érable, orienté à gauche; en bas motif de feuilles de lierre, orienté à gauche. Frise de Philarcrrvs.

Frise 48 : en haut motif de feuilles dentelées nervurées, orienté à gauche; en bas motif de feuilles de lierre, orienté à gauche. Frise non attribuée.

Frise 49: en haut motif indéterminé orienté à gauche; cn bas motif de feuilles de lierre, orienté à gauche. Frise non attribuée.

Frise 5(): en haut motif de feuilles opposées avec deux bourgeons latéraux, orienté à droite; en bas motif de petits fleurons à huit pétales. Frise de T. Cavivs. 
Il est intéressant de noter que très peu de frises arec des compositions semblables se retrouvent che\% deux potiers. Encore faut-il remarquer que dans ce cas, les motifs proviennent de poinçons différents, permettant de les distinguer (par exemple frise 7 de Chrysippvs et frise 8 d'Hilarvs) ou que le même motif est utilisé mais dans un autre sens, par exemple avec le motif vers la droite pour Chrysippvs (frise 14) et vers la gauche pour T. Cavivs (frise 13). Ce constat permet d'attribuer avec une forte probabilité un élément de frise à un décorateur, même en l'absence de signature. Seules six frises n'ont pu être attribuées à un fabricant (frises 5, 16, 32, 36, 48, 49). Les quarante-quatre autres se répartissent entre les différents potiers de la manière suivante :

\section{ACO : 2}

HIIARVS : 18

CHRYSIPPVS : 22

PHILARCVRVS : 5

PHILOCRATES : 1

T. CAVIVS : 4

FIDELIS : 1

On notera que sur les quarante-quatre frises, huit frises seulement $(1,10,13,14,15,21,29$ et 38) sont communes à plusieurs potiers. Toutes les frises se distinguent aisément de celles de l'atelier de Loyasse. En revanche, vingt-deux d'entre elles sont présentes dans les productions de l'atelier de Saint-Romain-en-Gal.

\section{LES DÉCORS}

L'étude du matériel a permis de reconstituer un grand nombre de décors et d'en attribuer la plupart avec certitude aux différents décorateurs, grâce aux signatures. Il a été possible également de distinguer pour un même décor l'utilisation de plusieurs moules, à partir de certains détails. Beaucoup de décors sont fragmentaires et ont été reconstitués à partir de fragments appartenant à différents gobelets. Nous avons appelé " décor » une composition associant différents poinçons. Une légère variation dans la disposition des poinçons est l'indice de l'existence d'un autre moule. Dans ce cas, le numéro du décor reste le même mais nous avons indiqué l'existence de deux moules (par exemple : décors $3 \mathrm{a}$ et $3 \mathrm{~b}$ ). En revanche, si l'un des éléments constitutifs du décor change, frise, signature ou poinçon décoratif, nous arons considéré qu'il s'agissait d'un autre décor, même si le schéma décoratif général reste le même (par exemple : décors 5 et 6).

\section{LES DÉCORS DE CHRYSIPPVS}

C'est de loin la production de Chrysipprs qui présente le plus grand nombre de décors (pl. 44 à 64). Ceux-ci montrent une grande variété, alliée à une richesse iconographique qui se traduit par l'utilisation de nombreux poinçons. On peut classer les décors en six catégories : décors de gladiateurs, de cirque, de métopes, de frises, décors composites et décors de semis de picots.

\section{Gobelets à décor de gladiateurs (pl. 44 à 51)}

Onze décors avec des combats de gladiateurs ont été distingués.

\section{Décor 1 (pl. 44)}

Sous une frise de type 20, des guirlandes séparées par des bouquets d'acanthe encadrent la signature de CHRYSIPPVS. Dans le champ, deux couples de gladiateurs séparés par deux couples de hérauts jouant de la trompette, le premier tourné vers la gauche est placé sous la signature, le second est tourné vers la droite. Les deux couples de gladiateurs présentent, à gauche de la signature, le combat d'un rétiaire et d'un Gaulois (?), à droite, le combat entre un mirmillon (?) et un gladiateur, avec le bouclier rond et la lance. À la base du décor, court une frise de dauphins tournés vers la gauche. Le moule d'où est tiré le décor présente un défaut caractéristique, une strie horizontale qui traverse tout le décor à $1,5 \mathrm{~cm}$ sous la frise.

Ce défaut permet d'identifier avec certitude le même moule dans l'atelier de Saint-Romain-en-Gal.

Décor 2 (pl. 44)

De composition voisine, ce décor montre sous une frise de type 28 des guirlandes séparées par des têtes encadrant la signature de CHRYSIPYVS. Sous la signature, un seul héraut sépare les couples de gladiateurs identiques. À la base, une frise de dauphins.

Une composition très voisine existe également à SaintRomain-en-Gal.

\section{Décor 3 (pl. 45)}

Sous une frise de type 28, des guirlandes séparées par des têtes. Sous la signature de CHRYSIPPVS, une palme verticale sépare les deux paires de gladiateurs : à gauche 
le Gaulois affronte le rétiaire ; à droite le mirmillon combat le Samnite? À la base : frise de bouquets d'acanthe.

Le même décor se rencontre à Saint-Romain-en-Gal mais avec un moule différent.

Un autre fragment appartient au même décor (3b) un héraut orienté vers la gauche, tourne le dos au rétiaire.

Ce fragment est issu du même moule que celui de Saint-Romain-en-(ial.

Décor 4 (pl. 45)

Ce décor présente une composition proche du précédent : même frise et même guirlande, mais la palme verticale sépare le Samnite à gauche d'un autre gladiateur à droite.

Décor 5 (pl. 46 et 47)

Sous une frise de type 1 , une frise de guirlandes avec des rinceaux se développe de part et d'autre de la signature de CHRYSIPPVS, encadrée de deux aigles. Le décor central associe une scène érotique à un combat de gladiateurs. Un couple sur un lit est encadré par deux personnages portant des récipients. À gauche de la scìne, un héraut. Le combat de gladiateurs montre l'affrontement de deux gladiateurs armés d'un glaive et d'un bouclier ovale. À la base du décor, une frise de feuilles.

Le même décor issu du même moule existe à SaintRomain-en-Gal.

Décor 6 (pl. 46)

De composition similaire au décor précédent, il sc distingue par la frise, de type 21 , et par le motif qui encadre la signature : un fleuron à huit pétales.

Décor 7 (pl. 48 et 49)

Sous une frise constituée de fleurs à six pétales (type 17), des guirlandes avec rinceaux encadrent la signature de CHRYSIPPVS. Trois paires de combattants s'affrontent : sous la signature, un rétiaire est affronté à un gladiateur avec le scutus et un casque à cimier. À leur gauche, s'opposent deux combattants armés d'une épée courbe, brandissant dans l'autre main une clochette. La présence de cette clochette, ainsi que la position de la tête des combattants, permet de les identifier comme des gladiateurs aveugles. L.e troisième combat met en présence des pugi- listes. À la base du décor, se développe une frise de rinceaux comme à la partie supérieure. Plusieurs fragments appartiennent à ce même décor, tiré d'un autre moule (7b). Il s'en distingue par la forme de la guirlande sous la signature de CHRYSIPPVS, ainsi que par la position des pugilistes.

Décor inconnu à Saint-Romain-en-Gal.

Décor 8 (pl. 50 et 51$)$

Sous une frise de type 17, la signature de CHRYSIPPVS apparaît flanquée d'une rosette, sous la signature, les pugilistes s'affrontent.

Décor inconnu à Saint-Romain-en-Gal.

Décor 9 (pl. 50 et 51$)$

Sous une frise de type 21 , se déroulent des guirlandes avec rinceaux, de part et d'autre de la signature de CHRYSIPPVS, encadrée de deux étoiles à huit branches. À gauche de cette signature, dans la guirlande, s'inscrit un motif en forme de trident. Sous la signature, un héraut tourné vers la gauche. À sa gauche, un couple d'archers affrontés. Derrière un des deux archers, un arbitre brandit sa baguette. À droite de la signature et du héraut, un autre couple de gladiateurs s'affronte : un mirmillon (?) au bouclier carré est opposé au gladiateur au bouclier rond et à la lance (Samnite?).

Ce décor est incommu à Saint-Romain-en-Gal.

Décor 10 (pl. 50 et 51)

De composition proche du décor précédent, il s’en distingue par l'absence du motif en forme de trident dans la guirlande, au-dessus d'un des archers. On note également que la trompette du héraut arrive sous le $\mathrm{R}$ et non sous le $\mathrm{H}$. Une autre différence est constituée par la position de l'arbitre dont la tête passe au-dessus de la guirlande, alors qu'elle est au-dessous dans le décor 10.

Décor inconnu à Saint-Romain-en-Gal.

Des fonds avec un décor de feuilles tournées vers le bas appartiennent très vaisemblablement aux décors 10 et 11 .

Décor 11 (pl. 5l)

Ce décor est tiré du seul moule de Chrysipprs, recueilli dans l'atelier. Le décor (positif) est le suivant : 
sous une frise de type 21 se développent des guirlandes avec rinceaux, de part et d'autre de la signature de CHRYSIPPVS, encadrée par deux étoiles à huit branches. Sous la signature un arbitre brandissant sa baguette qui traverse une des étoiles, sépare deux couples de gladiateurs : à droite un mirmillon affronte un Samnite, à gauche deux archers s'opposent, un héraut sépare les deux paires de gladiateurs, à l'opposé.

Décor inconnu à Saint-Romain-en-Gal.

\section{Gobelets à décor de cirque (pl. 51)}

Seuls quelques fragments se rapportent à des gobelets à décor de cirque, connus par des spécimens de SaintRomain-en-Gal (Desbat, 1985). Un seul décor est identifiable, mais il provient de deux moules diffërents.

\section{Décor 12 (pl. 51)}

Un aurige brandissant le fouet conduit un quadrige. Les roues d'un autre quadrige apparaissent devant les jambes des chevaux. Au-dessus des chevaux d'un quadrige apparaît un aigle. Sur un autre fragment encore, apparaît une des metae du cirque avec un héraut tourné vers la droite.

Décor connu à Saint-Romain-en-Gal, tiré du même moule.

Gobelets à décor de métopes (pl. 52 à 56)

Il s'agit de gobelets dont le décor se développe sur deux registres. Un registre supérieur divisé en métopes par des colonnes et des guirlandes et un registre inférieur avec des motifs en sautoir séparés par des bandes verticales, constituées de différents motifs.

\section{Décor 13 (pl. 52 et 53)}

Sous une frise de type 14, le registre supérieur est divisé par sept colonnes reliées par des guirlandes. Audessus des guirlandes sont disposés des épis de blé et un trident. Un rang de perles fait la séparation avec le registre inférieur, divisé par sept bandes qui alternent des motifs de fleurons et de disques, surmontées de bustes, dans lesquels on reconnaît Auguste, Agrippa, Minerve ou Rome. Entre les bandes de motifs encadrées par les lignes de perles, des croix de Saint-André formées par des lignes de perles limitent des triangles frappés de fleurons et d'un dauphin à la partie supérieure. Les métopes sont de largeur variable. La plus étroite ne comporte pas de dauphin au-dessus de la croix de Saint-André.

Ce décor est connu à Saint-Romain-en-Gal avec la signature CHRYSIPPVS, issu du même moule.

\section{Décor 14 (pl. 52)}

Sous une frise de type 19, le registre supérieur est divisé en métopes par des colonnes surmontées de dauphins, entre les colonnes des guirlandes, sous les guirlandes des couples de dauphins affrontés. Une frise de fleurons sépare les deux registres. Le registre inférieur est divisé par des bandes de fleurons alternant avec des motifs végétaux, encadrées par des lignes de perles. Entre les bandes, des lignes de perles forment des croix de Saint-André frappées en leur centre par un fleuron.

Décor inconnu à Saint-Romain-en-Gal.

\section{Décor 15 (pl. 54)}

Sous une frise de type 20, le registre supérieur est divisé par des colonnes supportant des guirlandes. La signature CHRYSIPPVS s'inscrit sous la frise entre deux colonnes. Un défaut du moule empâte le I. Au-dessus des guirlandes sont disposés des dauphins, une bande de fleurons sépare les deux registres. Le registre inférieur est divisé par des bandes verticales de motifs végétaux.

Le même décor se rencontre à Saint-Romain-en-Gal, mais avec un autre moule, sans le défaut de la signature.

\section{Décor 16 (non représenté)}

Plusieurs fragments appartiennent à un décor proche. Sous une frise identique la signature de CHRYSIPPVS surmonte un dauphin tourné vers la droite.

\section{Décor 17 (pl. 54)}

Sous une frise double de type 37 , le registre supérieur est divisé par des colonnes supportant des guirlandes. Audessus des guirlandes sont disposées des couronnes. Une ligne de fleurons sépare les deux registres. Au registre inférieur incomplet un masque sous la colonne et un aigle disposé au-dessus d'une croix de Saint-André. Un autre moule comporte le même décor. Il se distingue du précédent par la position des lettres de la signature.

Décor inconnu à Saint-Romain-en-Gal. 


\section{Décor 18 (pl. 54)}

Sous une frise de type 7, le registre supérieur est divisé par des colonnes supportant des guirlandes. Au-dessus des guirlandes sont disposés des couronnes et des trophées, la signature de CHRYSIPPVS s'inscrit entre deux colonnes, au-dessus d'une couronne.

Décor inconnu à Saint-Romain-en-Gal.

\section{Décor $19(\mathrm{pl} .55)$}

Sous une frise double de type 37 , le registre supérieur est divisé par des colonnes surmontées par des dauphins et supportant une guirlande. Au-dessus de la guirlande, masque de face. La signature de CHRYSIPPVS s'inscrit entre deux colonnes au-dessus d'un masque. Le registre inférieur manque.

Décor présent à Saint-Romain-en-Gal.

Décor 20 (pl. 55)

Sous une frise de type 19 , le registre supérieur est divisé par des colonnes surmontées de têtes et de masques. Au-dessus des guirlandes reliant les colonnes, sont disposés des dauphins. Sous la signature de CHRYS (ippvs) figure un masque. Une ligne de fleurons sépare les deux registres. Au registre inférieur, des bandes de fleurons sont disposées sous les colonnes.

Décor inconnu à Saint-Romain-en-Gal.

\section{Décor 21 (pl. 55)}

Sous une frise de type 20, le registre supéricur cst divisé par des colonnes, entre les colonnes sont placées diverses figures mythologiques : Fortune, Minerve, Vénus... Entre deux colonnes s'inscrit la signature de (Chrysi) PPVS. Une frise de fleurons sépare les deux registres. Au registre inférieur, des bandes de fleurons verticales sont séparées par des croix de Saint-André ellesmêmes formées de fleurons. Dans le triangle supéricur est disposé un aigle et dans l'inférieur une feuille.

Décor inconnu à Saint-Romain-en-Gal.

Décor 22 (pl. 55)

Sous une frise de type 10 , le registre supérieur est divisé par des colonnes, supportant des guirlandes. Audessus des guirlandes, des aigles. La signature de
CHRYSIPPVS s'inscrit entre deux colonnes supportant des dauphins. Sous la signature, le buste de Vénus.

Décor inconnu à Saint-Romain-en-Gal.

Décor 23 (pl. 55)

Sous une frise de type 10 , le registre supérieur est divisé par des colonnes avec des chapiteaux à feuilles d'acanthe. Entre les colonnes, bustes ou figures mythologiques. La signature de CHRYSIPPVS s'inscrit entre deux colonnes supportant des masques affrontés. Au registre inférieur séparé par une ligne de fleurons, motif de croix de Saint-André. Sous la colonne un motif de «vires ».

Décor inconnu à Saint-Romain-en-Cal.

Décor 24 (pl. 56)

Sous une frise double de type 37 , la signature de CHRYSIPPVS s'inscrit entre des colonnes surmontées d'acanthe qui divisent la frise supérieure. Entre les colonnes, des figures sont disposées deux à deux, Minerve et Victoire sous la signature, masque, Victoire et personnage féminin. Au-dessous, des paires de dauphins affiontés sont séparés par un épi de blé.

Décor inconnu à Saint-Romain-en-Gal.

Décor 25 (pl. 56)

Sous une frise de type 21, le registre supérieur est divisé par des colonnes surmontées d'un bouquet d'acanthe, des guirlandes joignent les chapiteaux. Au-dessus de la guirlande figure un masque. Ia signature de CHRYSIPPVS s'inscrit au-dessus d'une guirlande, sous laquelle est placée une Vénus au miroir. Au registre inférieur, une rangée de triangles de picots pointe en haut.

Décor inconnu à Saint-Romain-en-Gal.

Gobelets à décor de frises (pl. 57 à 60)

Ces gobelets se caractérisent par une frise continue surmontant un registre inférieur avec motifs disposés en bandes verticales alternés avec des croix de Saint-André, ou bien des décors couvrants constitués de motifs végétaux. 
Décor 26 (pl. 57)

Sous une frise de type 21, la signature de CHRYSIPPVS encadrée par une Vénus au miroir et un monstre marin, est interrompue par une Fortune. De part et d'autre, la frise comporte différents motifs : épi de blé, masque, Vénus, dauphins et trophée, aigle, Minerve, buste d'Auguste, Minerve, buste de Vénus, Victoire. Au registre inféricur, quatre bandes verticales de fleurons surmontées de masques alternent avec des croix de Saint-André. Au-dessus de celles-ci s'inscrivent quatre compositions de plusieurs poinçons : grandes feuilles surmontant un dauphin, entre deux trophées, Éros entre deux dauphins, épi de blé entre deux poissons, trident surmontant une couronne entre deux aigles.

Le même décor existe dans l'atelier de Saint-Romainen-Gal, avec le même moule.

\section{Décor 27 (pl. 58)}

Sous une frise de type 27, une frise continue se compose d'oiseaux picorant une grappe de raisin, en alternance avec d'autres motifs : aigle, Minerve, Vénus au miroir, Victoire, Éros, aigle, trophée. Ln fragment appartenant au même décor livre la signature (Chry) SIP (pvs) au-dessus d'une femme à sa toilette. Au registre inférieur séparé par une ligne continue, des épis de blé alternent arec des bandes verticales de disques surmontées de masques ou du buste d'Auguste et Vénus.

Le même décor existe à Saint-Romain-en-Gal.

Décor 28 (pl. 58)

Sous une frise de type 29, la signature de (Ch) RYSI (ppvs) s'inscrit de part et d'autre d'une Fortune, au-dessus de grappes de raisin. Au registre supérieur, une frise continue se compose d'oiseaux picorant une grappe de raisin, en alternance avec des motifs d'épi de blé, Éros, Vénus à sa toilette. Le registre inférieur séparé par une ligne de perles est divisé par des bandes verticales surmontées de masques de face... Entre les bandes verticales des bandes de perles disposées en croix délimitent des triangles ornés de différents motifs : trophée, aigle, poissons.

Le même décor existe à Saint-Romain-en-Gal.
Décor 29 (pl. 58)

Sous une frise de type 33, la signature de CHRYSIPPVS s'inscrit entre deux aigles au-dessus de triangles de picots, la pointe vers le bas. De part et d'autre se déroule une frise de losanges constitués de picots. Au registre inférieur, divers motifs séparés par des bandes de disques superposés surmontées par des couronnes : Victoire, buste d'Octave, monstre marin, Minerve et deux taureaux cornupètes affrontés.

Décor connu à Saint-Romain-en-Gal.

Décor 30 (pl. 58)

Sous une frise de type 29, la signature de CHRYSIPPVS s'inscrit au-dessus d'une ligne de triangles de picots, la pointe vers le bas. Au registre inférieur, le buste de Vénus surmonte une bande verticale de disques, bordée de rang de perles, de part et d'autre un poisson et, à gauche, un dauphin.

Décor inconnu à Saint-Romain-en-Gal.

Décor 31 (pl. 58)

Sous une frise de type 14, un gorgoneion est accroché à un motif de double feuille. Ce décor n'est connu que par un seul fragment, mais la frise particulière le distingue de tous les autres. Malgré l'absence de signature, le style permet de l'attribuer à Chrysippvs.

Décor inconnu à Saint-Romain-en-Gal.

Décor 32 (pl. 59)

Sous une frise de type 42 , le registre supérieur est divisé par des colonnes alternant avec différents sujets : Éros, Minerve, Victoire. Sous les personnages des dauphins tournés vers la gauche. Le registre inférieur, séparé par une ligne de perles, est orné d'un décor couvrant de feuilles imbriquées. La signature de (Chrysip) PVS s'inscrit sous la première ligne de la frise double.

Décor inconnu à Saint-Romain-en-Gal.

Décor 33 (pl. 59)

Sous une frise de type 21, le registre supérieur est orné d'une frise décorée de masques, placés au-dessus de bouquets d'acanthe en alternance avec des figures de divinités. La signature de CHRYSIPPVS s'inscrit sous la frise 
entre deux masques affrontés. La frise est séparée par un rang de perles de la panse qu'il faut restituer ornée d'un décor couvrant de feuilles de lierre.

Décor connu à Saint-Romain-en-Gal.

\section{Décor 34 (pl. 59)}

Sous une frise de type 20, la signature de CHRYSIPPVS s'inscrit entre deux feuilles, au-dessus d'une guirlande. Le registre supérieur est orné de différents motifs, oiseaux sur des grappes de raisin, masque, gorgoneion, Victoire, lièvre mangeant des raisins, alternant avec des demi-colonnes supportant des masques. Le registre inférieur, séparé par un rang de perles, est orné d'un semis de fleurons, dans lequel s'inscrit un croissant de lune.

Ce décor est inconnu à Saint-Romain-en-Gal.

\section{Décor 35 (pl. 60)}

Sous une frise de type 13, la signature de CHRYSIPPVS s'inscrit entre deux dauphins, au-dessus de poissons. Sous le dauphin de gauche figure un trident. De part et d'autre, la frise se compose de masques posés sur des demi-colonnes, qui alternent avec diverses figures, lapin et Éros, oiseau picorant des raisins, Minerve tenant une Victoire... Une ligne de feuilles doubles sépare la frise du registre infërieur orné d'un décor couvrant de feuilles de lierre imbriquées.

Décor connu à Saint-Romain-en-Gal.

Un gobelet avec le même décor existe au Lorenzberg (Ulbert, 1965, pl. 25).

\section{Décor 36 (pl. 60)}

La frise manque. Le registre supérieur se compose de demi-colonnes surmontées de masques, alternant avec des motifs de lapins et d'oiseaux mangeant des raisins. Sur un fragment une composition associe une grande feuille avec une grappe de raisins. Une ligne de perles fait la séparation avec le registre inférieur orné d'un décor courant de motifs imbriqués.

Décor inconnu à Saint-Romain-en-Gal.

Décor 37 (pl. 60)

Sous une frise de type 21, la signature de CHRYSIPPVS s'inscrit entre deux masques. Au-dessous se déroule une frise où l'on reconnaît un cavalier au bouclier rond, et une composition associant Harpocrate entre deux Victoires, dont l'une tient un trophée et brandit une couronne, à sa gauche figure l'arrière-train d'une panthère.

Ce décor est très proche de celui d'un gobelet de Saint-Romain-en-Gal (SRG 25). Le parallèle avec le décor de Saint-Romain-en-Gal permet de considérer que celuici se termine à la base par un semis de feuilles de lierres imbriquées.

\section{Gobelets à décor composite}

Décor 38 (pl. 53 et 61)

Sous une frise de type 21, la signature de CHRYSI (ppvs) s'inscrit entre des vires au-dessus de triangles de picots. De part et d'autre se déroulent deux lignes de losanges de picots. En dessous, la panse est divisée en quatre parties par des bandes verticales de disques, surmontées de colonnes, supportant le buste de Vénus, un masque et un scarabée; chaque champ délimité par les bandes verticales est orné d'une résille formée de lignes de perles. Dans la partie supérieure s'ordonnent divers motifs : Vénus et dauphin, Minerve entre deux couronnes, au-dessus d'un dauphin, Fortune entre deux couronnes au-dessus d'un trophée, Victoire entre deux couronnes au-dessus de deux poissons. Dans la partie gauche des métopes apparaissent d'autres motifs : personnage, trident et aigle.

Le moule présentait des défauts qui apparaissent dans le décor sous la forme de lignes brisées. Le même décor avec les mêmes défauts est connu à Xanten. Décor inconnu à Saint-Romain-en-Gal.

Décor 39 (pl. 61)

Ce décor incomplet n'est attesté que par un seul fragment. Sous la signature de CHRYSI (ppvs) apparaît un trophée et une Victoire.

Décor inconnu à Saint-Romain-en-Gal.

\section{Les décors à semis de picots}

Ce décor couvrant caractéristique des gobelets d'Aco a été également utilisé par Chrysippvs. Seuls trois gobelets avec ce décor ont été reconstitués (pl. 61,62 et 64) mais l'étude des signatures et des frises permet d'évaluer le nombre de moules avec ce type de décor à dix-sept exemplaires. 
Décor 40 (pl. 61, 62 et 64)

Sous une frise de type 20, la signature de CHRYSIPPVS s'inscrit entre deux coquilles Saint-Jacques. Ce décor a été réalisé avec trois moules :

- 40a : le C de Chrysipprs touche la coquille; un seul picot sous le $\mathrm{C}$,

- 40b : le C ne touche pas la coquille, mais touche le $\mathrm{H}$; deux picots sous le $\mathrm{C}$,

- 40c : le Cine touche ni la coquille, ni le H.

Décor comnu à Saint-Romain-en-Gal, arec les mêmes moules.

Décor 41 (pl. 61 et 64)

Sous une frise de type 20, la signature de CHRYSIPPVS s'inscrit entre deux coquilles Saint-Jacques. Sous la signature les picots sont disposés en triangle.

Décor inconnu à Saint-Romain-en-Gal.

Décor $42(\mathrm{pl} .62$ à 64)

Sous une frise de type 22, la signature de CHRYSIPPVS s’inscrit entre deux fleurons à huit pétales.

Comme le décor 10, celui-ci a été réalisé avec plusieurs moules :

- 42a : la queue de la feuille de la frise commence à la hauteur du (;,

- 42b : la queue de la feuille de la frise se situe entre le fleuron et le $C$,

- 42c : la queue de la feuille commence à la hauteur du C. mais la feuille suivante est décalée.

Décor connu à Saint-Romain-en-Cal.

Décor 43 (pl. 64)

Sous une frise de type 28 , la signature de CHRYSIPPVS s'inscrit entre deux feuilles de lierre.

Décor connu à Saint-Romain-en-Gal.

Décor 44 (pl. 64)

Sous une frise de type 20 , la signature de CHRYSIPPVS s’inscrit au-dessus d'une guirlande, entre deux masques.

Décor connu à Saint-Romain-en-Gal.
Décor 45 (pl. 64)

Sous une frise de type 13, la signature de CHRYSIPPVS s'inscrit au-dessus d'une guirlande, entre deux masques.

Décor comnu à Saint-Romain-en-Caal.

Décor 46 (pl. 64)

Sous une frise de type 7 , la signature de (HRYSIPPVS s'inscrit entre deux feuilles de licre.

Décor commu à Saint-Romain-en-Cial.

Décor 47 (pl. 64)

Sous une frise de type 18 , la signature de CIIRYSIPPVS s’inscrit entre deux feuilles de lierre.

Décor connu à Saint-Romain-en-Gal, avec le môme moule.

Décor 48 (pl. 64)

Sous une frise de type 11 s'inscrit la signature de CHRYSIPPVS.

Décor connu à Saint-Romain-en-Càl.

Décor 49 (pl. 64)

Sous une frise de type 26, la signature de CHRYSIPPVS s'inscrit sous la forme CHRYSIPPVS/ARS, disposée entre deux masques; quatre feuilles verticales sont disposées sous le nom de Chrysipprs, de part et d'autre de "ars ". À la base du décor de picots sont aménagés des triangles en réserve garnis d'une feuille verticale, du même tỵpe que celle qui encadre la signature.

Il faut remarquer que la signature CHRYSIPPVS semble frappée avec un poinçon unique à la différence des autres gobelets où la signature est obtenue en imprimant chaque lettre séparément. Il pourrait donc s'agir d'un poinçon obtenu par surmoulage. Il est donc possible que le réalisateur du moule ne soit pas Chrysipprs. Décor connu à Saint-Romain-en-Cal, issu du même moule.

LES DÉCORS D'ACO (pl. 66)

Seuls deux décors sont atribuables à Aco. Ils se limitent tous les deux au décor de semis de picots surmonté d'une frise végétale. 


\section{Décor 1}

Dans une frise de type 35 s'inscrit la signature ACO.

Décor connu à Saint-Romain-en-Gal.

\section{Décor 2}

Au-dessus d'une frise double de type 41, sont disposés à intervalles réguliers plusieurs masques tournés vers la gauche, au sommet du décor de picots. Dans l'espace entre deux masques, la signature ACO s'inscrit, sous la première rangée de picots.

Décor inconnu à Saint-Romain-en-Gal.

LES DÉCORS D'Hilarvs ACo (pl. 62, 63 et 65 à 67, 71)

Comme ceux d'Aco, les décors d'Hilarvs Aco ne comportent aucune scène figurée en dehors des motifs qui encadrent la signature et tous présentent le décor de semis de picots surmonté d'une frise végétale. L'association entre les signatures, les frises et les motifs d'encadrement (voir signatures) permet d'attribuer à Hilarvs vingt-huit moules. Il faut sans doute y ajouter un dernier décor représenté par plusieurs fragments qui montrent un couple de gladiateurs dans un semis de picots. Aucune signature n'a pu être mise en relation mais un fragment permet d'identifier une frise de type 1 et le même décor se retrouve sur un gobelet d'Oberaden signé Hilarvs associé précisément à la frise 1 (Loeschcke, 1942, pl. 4, 2 et pl. 28, 34).

Décor 1 (pl. 66)

Sous une frise de type 1, le nom d'HILARVS seul s'inscrit au sommet du décor de picots. À ce décor appartiennent vraisemblablement les deux gladiateurs inscrits dans un semis de picots, et peut-être Minerve.

Décor inconnu à Saint-Romain-en-Gal.

Décor 2 (pl. 66)

Sous une frise de type 31, le nom d'HILARVS scul s'inscrit au-dessus du décor de picots, sans motif d'encadrement.

Décor inconnu à Saint-Romain-en-Gal.
Décor 3 (́pl. 66)

Sous une frise double de type 40 la signature HILARVS seul s'inscrit entre deux masques tournés vers la gauche, au sommet du décor de semis de picots.

Décor inconnu à Saint-Romain-en-Gal.

Décor 4 (pl. 66)

Sous une frise double de type 46, le nom d'HILARVS apparaît entre deux abeilles, au sommet du décor de picots.

Décor inconnu à Saint-Romain-en-Gal.

Décor 5 (pl. 66)

Sous une frise de type 9 , la signature P/HILARVS sans encadrement s'inscrit au sommet du semis de picots.

Décor connu à Saint-Romain-en-Gal.

Décor 6 (pl. 66)

Sous une frise de type 3 , la signature HILAR ACO s'inscrit au sommet du décor de semis de picots.

Décor inconnu à Saint-Romain-en-Gal.

Décor 7 (pl. 66)

Sous une frise de type 8 , la signature HILARVS ACO s'inscrit sans motif d'encadrement au sommet d'un décor de semis de picots. Un picot sépare Hilarvs et Aco.

Décor inconnu à Saint-Romain-en-Gal. Ce décor se retrouve sur quatre moules, identifiables à certains détails :

- 7a : le H de Hilarrs est plus bas que la ligne de picots supérieure. La signature est à $2 \mathrm{~mm}$ sous la frise. Le $\mathrm{H}$ est différent des autres moules,

- 7b : le H de Hilarvs est au même niveau que la ligne de picots supérieure. La signature est à $1 \mathrm{~mm}$ sous la frise,

- 7c : le $\mathrm{H}$ de Hilarvs est au même niveau que la ligne de picots supérieure mais la signature penche de $1 \mathrm{~mm}$, sous la frise à $2 \mathrm{~mm}$. Les picots sont plus petits que pour $7 \mathrm{~b}$,

- $7 \mathrm{~d}$ : le $\mathrm{H}$ de Hilarvs est au même niveau que la ligne supérieure de picots, mais la signature se situe à $2 \mathrm{~mm}$ de la frise et à $2 \mathrm{~mm}$ des picots situés en dessous. 
Décor 8 (pl. 66)

Sous une frise de tvpe 12, la signature HII ARVS ACO s'inscrit sans motif d'encadrement au sommet du décor de semis de picots. Un picot sépare Hilarvs et Aco.

Décor connu à Saint-Romain-en-Gal.

Décor 9 (pl. 66)

Sous une frise de type 31 , la signature HII ARVS ACO s'inscrit, sans motif d'encadrement, au sommet du décor de picots. Un picot sépare Hilarvs et Aco.

Décor inconnu à Saint-Romain-en-Gal.

Décor 10 (pl. 63 et 66)

Sous une frise de type 31, la signature HILARVS ACO s'inscrit entre deux feuilles bilobées, au sommet du décor de picots. Un picot sépare Hilarvs et Aco. À la base du décor de picots des fleurons à huit pétales s'inscrivent dans des triangles en réserve.

Décor inconnu à Saint-Romain-en-Gal.

Décor 11 (pl. 66)

Sous une frise de type 29 , la signature HIL ARVS ACO s'inscrit sans motif d'encadrement au sommet du décor de picots. Un picot sépare Hilarvs et Aco.

Décor connu à Saint-Romain-en-Gal.

Décor 12 (pl. 66)

Sous une frise de type 29, la signature HILARVS ACO s'inscrit entre deux fleurons à six pétales, au sommet du décor de picots.

Décor connu à Saint-Romain-en-Gal, avec un moule différent.

Décor 13 (pl. 66)

Sous une firise de type 21 , la signature HII ARIS ACO s'inscrit entre deux coquilles Saint-Jacques, au sommet du décor de picots. Un picot sépare Hilarvs et Aco.

Décor inconnu à Saint-Romain-en-Gal.
Décor 14 (pl. 66)

Sous une frise de type 9, la signature HILARVS ACO s’inscrit au sommet du décor de picots. Un seul fleuron encadre à gauche la signature. Un autre fleuron à six pétales sépare Hilarvs et Aco.

Décor inconnu à Saint-Romain-en-Gal.

Décor 15 (pl. 67)

Sous une frise de type 15 , la signature HII ARVS ACO s'inscrit entre deux feuilles bilobées, au sommet du décor de picots. Un picot sépare Hilarvs et Aco.

Décor inconnu à Saint-Romain-en-Gal.

Décor 16 (pl. 67)

Sous une frise de type 15 , la signature d'HILARVS ACO s'inscrit entre deux feuilles bilobées. Un point sépare Hilarvs et Aco. Le décor est tiré de deux moules :

- 16a : la partie inférieure du S d'Hilarvs se place entre deux picots,

- 16b : le S d'Hilarvs se place au-dessus d'un picot.

Décor connu à Saint-Romain-en-Gal.

Décor 17 (pl. 67)

Sous une frise de type 15 , la signature (Hilarv) S ACO s'inscrit sans motif d'encadrement. Un picot sépare Hilarvs et Aco.

Décor inconnu à Saint-Romain-en-Gal.

Décor 18 (pl. 62 et 67$)$

Sous une frise de type 24, la signature HII ARVS (Aco) s'inscrit entre deux feuilles bilobées. Le décor se rencontre avec deux moules.

Décor inconnu à Saint-Romain-en-Gal.

Décor 19 (pl. 67)

Sous une frise de type 15 , la signature HILARVS ACO s’inscrit au-dessus d'une guirlande, entre deux abeilles. Pas de motif de séparation entre Hilars et Aco.

Décor inconnu à Saint-Romain-en-Gal. 
Décor 20 (pl. 63 et 67)

Sous une frise de type 14, la signature HILARVS ACO s'inscrit entre deux coquilles Saint-Jacques. Pas de motif de séparation entre Hilarvs et Aco. À la base du décor, un motif de feuilles s'inscrit dans des triangles en réserve.

Décor inconnu à Saint-Romain-en-Gal.

\section{Décor 21 (pl. 67)}

Sous une frise double de type 38 , la signature HIL (arvs) s'inscrit entre deux fleurons à huit pétales.

Décor inconnu à Saint-Romain-en-Gaal.

Décor 22 (pl. 67)

Sous une frise double de type 39 , la signature HI (larvs) s'inscrit entre deux fleurons à huit pétales (frise interrompue).

Décor inconnu à Saint-Romain-en-Gal.

Décor 23 (pl. 67)

Sous une frise de type 21, la signature HILARVS ACO s'inscrit entre deux feuilles de lierre. Un picot sépare Hilarvs et Aco.

Décor inconnu à Saint-Romain-en-Gal.

Décor 24 (pl. 65 et 67)

Sous une frise de type 24, la signature HILARVS ACO s'inscrit entre Minerve et le buste de Sol. Picot entre Hilarvs et Aco.

Décor inconnu à Saint-Romain-en-Gal.

Décor 25 (pl. 67)

Sous une frise de type 24, la signature HII ARVS ACO s'inscrit entre deux masques, au-dessus d'une guirlande. Pas de séparation entre Hilarvs et Aco.

Décor inconnu à Saint-Romain-en-Gal.

Décor 26 (pl. 67)

Sous une frise de type 44, la signature HILAR (vs) (Aco)? s'inscrit entre deux masques.

Décor connu à Saint-Romain-en-Gal.
Décor 27 (pl. 67)

Sous une frise de type 29, la signature (Hil) ARVS ACO, motif d'encadrement? Un picot sépare Hilarvs et Acu.

Décor inconnu à Saint-Romain-en-Gal.

\section{Décor 28 (pl. 67)}

La frise manque. La signature (Hilar) VS est suivie d'un motif à double crosse.

Décor inconnu à Saint-Romain-en-Gal.

Décor 29 (pl. 67)

Sous unc frisc de type 25, la signature (Hila) RVS ACO s'inscrit entre deux (?) feuilles de lierre, orientées vers le bas. Un picot sépare Hilarvs et Aco. Ce décor n'est connu que par un fragment de moule.

Décor inconnu à Saint-Romain-en-Gal.

\section{Les déCors de Philarcvrvs (pl. 68)}

Quatre décors sont attribuables à Philarcvrvs, et un cinquième comporte la signature (Philar) CVRVS CAVIVS. Ces décors se différencient nettement de ceux de Chrysippvs ou d'Hilarvs $\Lambda$ co.

Décor 1 (pl. 68)

Dans une frise de type $47 \mathrm{~s}$ 'inscrit la signature de PHILARCVR (vs), au-dessus d'un décor constitué de lignes de feuilles de lierres disposées verticalement, en alternance avec des fleurons à douze pétales disposés au sommet d'une tige.

Décor 2 (pl. 68)

Sous une frise de type 45 , le décor se compose de rangées de fleurons à douze pétales disposés de part et d'autre d'une tige, en alternance avec un motif en forme de losange placé au sommet d'une tige. La signature de PHILARCV-RVS s'inscrit de part et d'autre d'une double rangée de fleurons.

Décor 3 (pl. 68)

Sous une frise de type 34, le décor se compose de rangées verticales de motifs alternés : feuilles de lierre entre 
deux lignes de perles et motif floral. La signature s'inscrit en trois parties PHILA/RCVR/VS séparées par des rangées de motifs.

Décor 4 (pl. 68)

Sous une frise de type 10, la signature (Philar) CVRVS suivie d'un picot s'inscrit au-dessus d'un décor de picots.

Décor 5 (pl. 65 et 69 )

Ce décor qui correspond à une forme basse se compose, sous une frise de type 2 , de lignes de perles obliques ou verticales. Sous la frise supérieure s'inscrit la signature (Philar) CVRVS CAVIVS.

Le même décor est connu dans le camp de Dangstetten (Fingerlin, 1986, 397, 18).

\section{LES DÉCORS DE T. CAVIVS}

Quatre décors uniquement sont attribuables à $\mathrm{T}$. CAVIVS (pl. 65).

Décor 1 (pl. 65 et 69$)$

Sous une frise de type 14, la signature T. CAVIVS s'inscrit entre deux masques dans un décor de semis de picots, avec des triangles en réserve à la base.

Le même décor est connu à Dangstetten (Fingerlin, 1986).

Décor 2 (pl. 65 et 69 )

Sous une frise de type 50, la signature de T. CAVIVS s'inscrit entre deux masques. Sur la panse, le décor se compose de trois registres de semis de picots : un premier registre formant pointe vers le bas est séparé du deuxième par une rangée de fleurons, et le deuxième est séparé du troisième par des losanges eux-mêmes formés de picots.

Ce décor se retrouve également dans le camp de Dangstetten (Fingerlin, 1986).

Décor 3 (pl. 65 et 69 )

Sous une frise de type 4, la signature T. CAVIVS s'inscrit entre deux masques, au-dessus d'un semis de picots couvrant la panse.
Ce décor n'est connu que par un fragment issu de la fouille de 1975 .

Décor 4 (pl. 69)

Sous une frise de type 29, la signature (T.) CAVIVS s'inscrit au-dessus du décor de picots. Motif d'encadrement inconnu.

\section{LE DÉCOR DE PHILOCRATES (pl. 70)}

Un seul décor est attribuable à Philocrates. Au-dessous d'une frise de type 15 s'inscrit la signature de PHILOCRAT (es). Dans le décor de semis de picots qui couvre la panse, sont ménagés des triangles en réserve dans lesquels sont placés des masques, ou une feuille de lierre. Un masque s'inscrit directement dans le décor de picots.

\section{LE DÉCOR DE FIDELIS (pl. 70)}

Un seul décor est attribuable à Fidelis. Sous une frise de type 30 , soulignée par une ligne, le nom de FIDELIS s'inscrit entre deux dauphins, au-dessus de deux rangs de feuilles, au centre d'une frise composée de masques. En dessous de cette frise se développe un décor de semis de picots.

\section{LES DÉCORS INDÉTERMINÉS OU NON ATTRIBUÉS (pl. 71)}

Plusieurs fragments appartiennent à un décor qu'il n'a pas été possible de rattacher avec certitude à un décorateur. Dans un champ de picots est inclue une figure de Minerve, le pied sur un bouclier. Le rapprochement avec le décor de gladiateurs attribuable à Hilarvs, pourrait toutefois suggérer qu'il s'agit d'une composition du même Hilarvs, voire un élément du même décor.

Décor 1 (pl. 71 et pl. 72, 12)

Sous une frise de type 16, se développe le décor de picots. Au même décor appartiennent des fonds qui présentent à la base une ligne de triangles de picots alternant avec des triangles en réserve ornés d'un fleuron. Au-dessus se développe une deuxième rangée de triangles alternés avec un motif de fleuron. Un filet en relief forme une séparation horizontale avec le semis de picots courrant le reste de la panse.

Décor inconnu à Saint-Romain-en-Gal. 


\section{Décor 2 (pl. 71)}

Sous une frise de type 32 , se développe le décor de picots. Forme haute.

Décor inconnu à Saint-Romain-en-(Gal.

\section{Décor 3 (non représenté)}

Sous une frise double de type 49 , se développe un décor de picots assez gros. Forme haute.

Décor inconnu à Saint-Romain-en-Gal.

\section{Décor 4 (non représenté)}

Sous une frise double de type 48 , se développe le décor de picots.

Décor inconnu à Saint-Romain-en-Gal.

Décor 5 (pl. 71)

Sous une frise de type 5, se développe le décor de picots. À la base, des motifs de feuilles de lierre sont placés verticalement dans des triangles en réserve. Forme basse. Le motif de feuille de lierre (poinçon 91) se retrouve sur le décor 1 de Philarcvrvs.

Décor inconnu à Saint-Romain-en-Gal.

Décor 6 (pl. 71)

Sous une frise de type 4 se développe le décor de semis de picots.

Décor de T. Cavivs?

\section{LES FONDS (pl. 72)}

Plusieurs fonds de gobelets à décor de picots qu'il est difficile de rattacher à un décor précis présentent des motifs disposés à la base du décor de picots. Le plus souvent, ces motifs s'inscrivent dans des triangles aménagés en réserve dans le semis de picots.

1. Fleuron à six pétales.

2. Feuilles bilobées disposées verticalement, le pétiole en haut.

3. Abeilles, disposées verticalement la tête en haut.

4. Feuille de lierre, la pointe en haut.

5. Motif en forme de gousse.

6. Frise continue de fleurons à six pétales.
7. Frise continue de feuilles bilobées tournées vers la droite.

8. Feuilles bilobées, avec le pétiole en haut, au-dessus d'une frise continue de fleurons à six pétales.

9. Fleurons à six pétales au-dessus d'une frise continue de feuilles de lierre, orientées vers la droite.

10. Frise continue de feuilles bilobées disposées horizontalement, le pétiole à gauche.

\section{INVENTAIRE DES POINCOONS}

L'étude des décors a permis de distinguer cent vingttrois poinçons.

\section{Les gladiateurs (pl. 73 et 74)}

Beaucoup de poinçons incomplets ont été reconstitués graphiquement à partir de plusieurs fragments.

La grande taille des motifs suggère que ceux-ci ont pu être réalisés à partir de plusieurs poinçons et que les têtes, les bras, les jambes, le subligaculum et les boucliers ont constitué des poinçons séparés. Cela aurait pu permettre de réaliser plusieurs motifs à partir de mêmes éléments, en changeant par exemple la tête ou le bouclier. On note en effet une grande similitude dans la position des motifs 2 et 7 . Toutefois cela n'est pas certain.

1. Rétiaire, hauteur $52 \mathrm{~mm}$. Le gladiateur avançant vers la droite tient un trident dans sa main gauche et un filet dans la droite. Ce dernier, enroulé autour de l'avant-bras, est relié par une corde au trident. Le gladiateur est fortement protégé. Il porte un casque avec des paragnathides, son bras gauche est protégé par la manica, et ses jambes par des ocreae, mais surtout son buste est couvert avec une cotte de mailles. Une telle représentation n'est pas habituelle pour un rétiaire, généralement figuré sans casque et le corps protégé uniquement par la manica. Peut-ètre s'agit-il d'un de ces "retiarii tunicati " signalés par Suétone. Ce motif entre dans la composition de plusieurs décors, associé au gladiateur 2 ou 3 .

Motif de Chrysippvs présent à Saint-Romain-en-Gal.

2. Gaulois? Hauteur $55 \mathrm{~mm}$. Ce gladiateur généralement affronté au rétiaire est tourné vers la gauche. Il brandit une épée et se protège avec un grand bouclier de type gaulois. Sa tête est couverte d'un casque avec paragnathides; des bandes de cuir (fasciae) protègent ses chevilles et ses poignets. Il est vêtu d'un pagne 
(subligaculum). L'épée longue et le grand bouclier suggèrent qu'il s'agit d'un Gaulois traditionnellement opposé au rétiaire.

Motif de Chrysipprs présent à Saint-Romain-en-Gal.

3. Samnite? Ce poinçon incomplet ne figure que sur le décor 7. Le gladiateur tourné vers la gauche brandit un bouclier long de type gaulois. Sa tête est coiffée d'un casque à cimier. Ce gladiateur est affronté au 1. Il peut s'agir d'une autre représentation de Gaulois.

Motif de Chrysippus inconnu à Saint-Romain-en-Cal.

4. Mirmillon? Hauteur $60 \mathrm{~mm}$. Gladiateur tourné vers la droite, vêtu d'un pagne seulement, il porte un bouclier carré et une épée courte, des jambières (ocreae) protègent ses tibias et ses genoux. Sa principale originalité réside dans son casque à visière et paragnathides, orné d'une figure difficilement identifiable. Il est tentant d'y voir un mirmillon, mais celui-ci derrait plutôt être opposé au rétiaire. Ce motif est opposé au 5 .

Motif de Chrysipprs présent à Saint-Romain-en-Gal.

5. Gladiateur à la lance. Hauteur $55 \mathrm{~mm}$. Ce gladiateur, tourné vers la gauche, n'est vêtu que d'un pagne. Sa tête est couverte par un casque à cimier avec paragnathides. Des jambières protègent ses tibias et ses genoux, des bandes de cuir (fasciae), ses poignets. Il tient un bouclier ovale ou rond de petite taille et une lance. Bien que son armement consiste en une lance, il ne peut s'agir d'un hoplomachus dont la première mention apparaît chez Suétone au $\mathrm{I}^{\mathrm{er}}$ s. après J.-C. Peut-être s'agit-il d'un Samnite ou d'un provocator dont on sait qu'ils combattaient quelquefois à la lance. Ce gladiateur est opposé au 4.

Motif de Chrysippss présent à Saint-Romain-en-Gal.

6. Gladiateur au bouclier ovale. Hauteur $56 \mathrm{~mm}$. Tourné vers la droite, il n'est vêtu que d'un pagne et brandit une épée courte dans sa main droite et un bouclier ovale. Sa tête est couverte d'un casque avec paragnathides. Des bandes de cuir (fasciae) protègent ses chevilles et ses poignets.

Motif de Chrysippss présent à Saint-Romain-en-Gal.

7. Gladiateur au bouclier ovale, tourné vers la gauche. Hauteur $55 \mathrm{~mm}$. Ce gladiateur présente le même armement que le 6 auquel il est affronté. Comme le précédent il est difficilement identifiable.

Motif de Chrỵsipprs présent à Saint-Romain-en-Gal.
8. Archer (sagitarius) tourné vers la droite. Il est vêtu d'un pagne, des bandes de cuir protègent ses chevilles. Sa tête est recouverte d'un casque avec paragnathides et un courre-nuque, différent de celui des autres gladiateurs. Son bras gauche est protégé par une manica dans laquelle sont fichées deux flèches. Sa main gauche tient un arc sur lequel sont engagées deux flèches, son bras droit pend le long du corps. Motif de Chrysippvs inconnu à Saint-Romain-en-Cal.

9. Archer (sagitarius) tourné vers la gauche. Ce second archer présente le même armement que le 7 auquel il est affronté.

Motif de Chrysipprs inconnu à Saint-Romain-en-Gal.

10. Gladiateur aveugle? tourné vers la droite. Vêtu seulement d'un pagne, il brandit une épée courte de la main droite et tient une clochette dans la main gauche. La tête manque.

Motif de Chrysippss inconnu à Saint-Romain-en-Gal.

11. Gladiateur aveugle tourné vers la gauche. Hauteur reconstituée $48 \mathrm{~mm}$. Vêtu comme le précédent il tient également une épée courte et une clochette. Sa tête couverte d'un casque à paragnathides est renversée en arrière. Le port de la tête ainsi que la présence de clochettes permet d'interpréter les motifs 10 et 11 comme des gladiateurs aveugles, qui préfiguraient les andabates, gladiateurs qui combattaient areuglés.

Motif de Chrysippvs inconnu à Saint-Romain-en-Gal.

12. Gladiateur tourné vers la droite. Hauteur : 21,5 mm (pl. 71). Vêtu d'une tunique serrée à la taille, il tient un bouclier long et une épée. Sa tête est couverte d'un casque à cimier. Ses jambes sont protégées par des ocreas.

Motif d'Hilarvs inconnu à Saint-Romain-en-Gal. Le poinçon est attribuable à Hilarvs grâce à un gobelet d'Oberaden, signé Hilarvs (Loeschcke, 1942, pl. 5, 17).

13. Gladiateur tourné vers la gauche. Hauteur : 22,5 mm (pl. 74). Il est vêtu comme le précédent auquel il est affronté. Il porte une tunique et son armement est identique.

Motif d'Hilarvs inconnu à Saint-Romain-en-Gal.

14. Gladiateur tourné vers la droite. Hauteur : $30 \mathrm{~mm}$. Il n'est vêtu que d'un pagne et porte un casque. Des ocreale protègent ses jambes, et son bras droit est protégé par des fasciae, il tient une épée courte. 
Poinçon attribuable à Chrysippvs, connu à SaintRomain-en-Gal.

\section{Autres personnages (pl. 74)}

15. Pugiliste. Motif incomplet tourné vers la droite. Motif de Chrysippvs inconnu à Saint-Romain-en-Gal.

16. Pugiliste. Motif incomplet tourné vers la gauche, affronté au 15.

Motif de Chrysippvs inconnu à Saint-Romain-en-Gal.

17. Arbitre. Hauteur : $49 \mathrm{~mm}$. Tourné vers la droite, il brandit sa baguette (rudis) de la main gauche. Il est vêtu d'une tunique courte serrée à la taille. Une écharpe ou cape courte flotte sur ses épaules.

Motif de Chrysippvs figurant dans le moule, inconnu à Saint-Romain-en-Gal.

18. Héraut. Hauteur : $43 \mathrm{~mm}$. Tourné vers la gauche et vêtu d'une tunique longue, il souffle dans une trompette.

Motif de Chrysippvs. Un motif semblable se retrouve à Saint-Romain-en-Gal sur un gobelet de Fidelis.

19. Héraut. Hauteur : $34 \mathrm{~mm}$. Tourné vers la gauche, il est vêtu d'une tunique s'arrêtant au-dessus du genou. Motif de Chrysippvs présent à Saint-Romain-en-Gal.

20. Héraut tourné vers la droite. Hauteur : $32 \mathrm{~mm}$.

21. Scène érotique. Largeur : $48 \mathrm{~mm}$. Couple sur un lit. Poinçon de Chrysippvs présent à Saint-Romain-en-Gal.

22. Aquarius. Hauteur : $21 \mathrm{~mm}$. Allant vers la gauche, il s'avance en tenant à bout de bras une cuvette.

Poinçon de Chrysippvs présent à Saint-Romain-en-Gal.

23. Aquarius. Hauteur : $27 \mathrm{~mm}$. Allant vers la droite. Les motifs 22 et 23 se rencontrent associés à la scène érotique 21 . On les retrouve également sur les courses de char.

Poinçon de Chrysippvs présent à Saint-Romain-en-Gal.

24. Cavalier chevauchant vers la gauche (non représenté). Motif incomplet figurant un cavalier casqué, tenant un bouclier rond.

Attribuable à Chrysippvs, présent à Saint-Romain-en-Gal.

25. Aurige. Motif incomplet (pl. 51).

Motif de Chrysippvs présent à Saint-Romain-en-Gal.

\section{Bustes et masques (pl. 75)}

26. Buste d'Octave Auguste. Hauteur : $12 \mathrm{~mm}$. La comparaison avec les types monétaires permet d'identifier sans l'ombre d'un doute ce motif avec une représentation d'Auguste jeune (fig. 79, 1-4).

Poinçon de Chrysippvs présent à Saint-Romain-en-Gal.
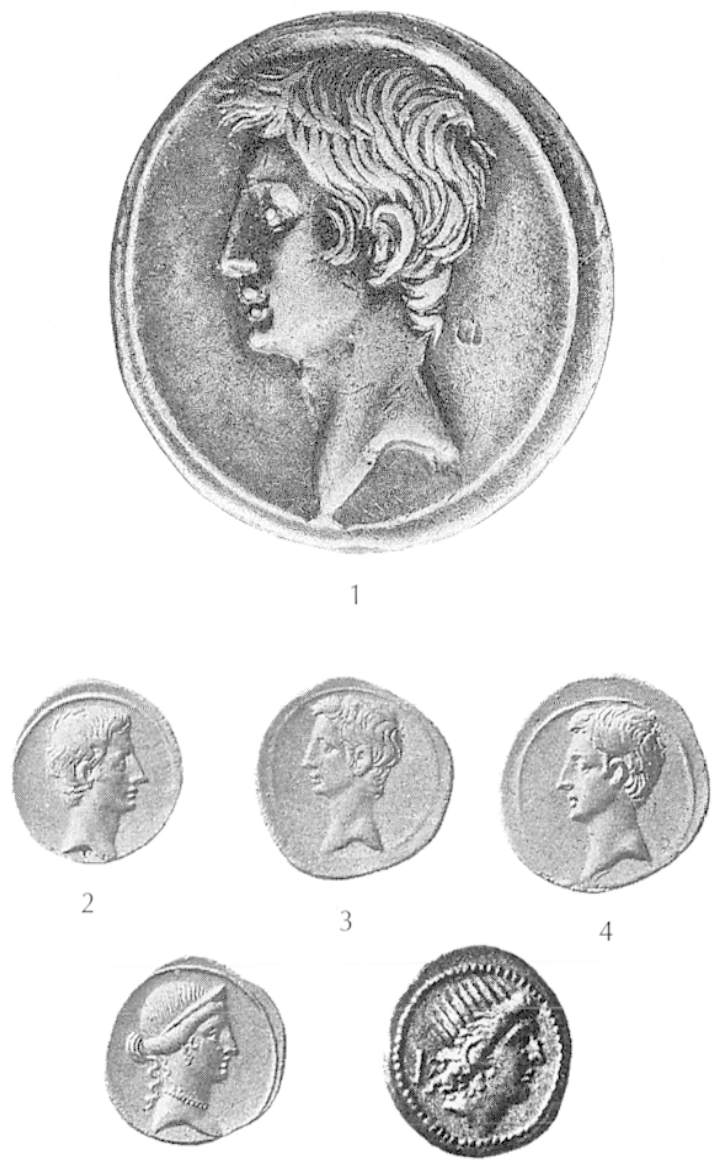

5

6

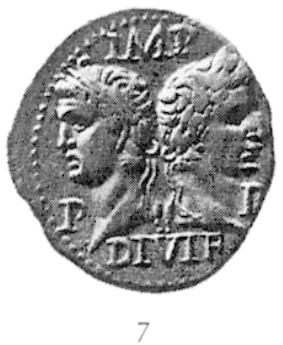

Fig. 79. Types monétaires présentant des ressemblances avec certains poincons de gobelets : 1-4, portraits d'Octave Auguste; 5, Vénus; 6. Sol; 7, as de Nîmes (échelle des $n^{\prime \prime} 2-7: 1 / 1$ ). 
27. Buste de Vénus. Hauteur : $12 \mathrm{~mm}$. Buste féminin tourné vers la gauche coiffé d'un chignon, un collier orne son cou. L'association de ce buste avec celui d'Auguste a pu faire penser qu'il s'agissait de celui de Livie, mais la comparaison avec un type monétaire permet de l'interpréter comme un buste de Vénus (fig. 79, 5).

Poinçon de Chrysipprs présent à Saint-Romain-en-Gal.

28. Buste d'homme. Hauteur : $14 \mathrm{~mm}$. Le troisième buste est celui d'un homme à cheveux courts tenus par un bandeau (tourné vers la gauche). Son association avec les portraits d'Auguste ainsi que la comparaison avec les as de Nîmes peut faire penser qu'il s’agit d'Agrippa (fig. 79, 6).

Poinçon de Chrysipprs prèsent à Saint-Romain-en-Gal.

29. Buste de Minerve ou de Rome. Hauteur : $10 \mathrm{~mm}$. Tête féminine casquée tournée vers la gauche.

Poinçon de Chrysippss présent à Saint-Romain-en-Gal.

30. Masque féminin. Hauteur : $9 \mathrm{~mm}$. Il est de profil, tourné vers la droite.

Poinçon de Chrysipprs présent à Saint-Romain-en-Cal.

31. Masque barbu de profil tourné vers la droite. Hauteur : $10 \mathrm{~mm}$. Tête de satyre?

Poinçon de Chrysippus présent à Saint-Romain-en-Gal.

32. Masque barbu de profil, tourné vers la droite. Hauteur : $11 \mathrm{~mm}$. Tête de satyre?

Poinçon de Chrysippus présent à Saint-Romain-en-Gal.

33. Masque barbu de profil, tourné vers la droite. Hauteur : $9 \mathrm{~mm}$. La barbe est courte, les chereux forment une calotte.

Poinçon de Chrysippus présent à Saint-Romain-en-Cal.

34. Masque barbu de profil, tourné vers la droite. Hauteur : $10 \mathrm{~mm}$.

Poinçon de Chrysipprs présent à Saint-Romain-en-Gal.

35. Masque imberbe de profil, tourné vers la droite. Hauteur : $13 \mathrm{~mm}$.

Poinçon de Chrysippvs présent à Saint-Romain-en-Gal.

36a. Masque féminin de profil, tourné vers la droite. Hauteur : $11 \mathrm{~mm}$. De longues boucles pendent derrière la tête.

Poinçon de Chrvsipprs présent à Saint-Romain-en-Gal. 36b. Même motif mais plus petit. Hauteur : $10 \mathrm{~mm}$. Ce poinçon a été probablement obtenu par surmoulage du précédent.

Poinçon de Fidelis inconnu à Saint-Romain-en-(;al.

37. Masque féminin de profil, tourné vers la gauche. Hauteur : $10 \mathrm{~mm}$.

Poinçon de Chrysippus présent à Saint-Romain-en-Gal.

38a. Masque féminin de profil, tourné vers la gauche. Hauteur : $10 \mathrm{~mm}$.

Poinçon d'Hilarvs présent à Saint-Romain-en-Caal.

38b. Même sujet, mais plus petit. Hauteur : $7,5 \mathrm{~mm}$.

Poinçon d'Hilarvs Aco. Ce motif se retrouve également sur des gobelets de Philocrates.

39. Masque féminin de profil, tournè vers la droite. Hauteur : $10 \mathrm{~mm}$.

Poinçon de T. Cavivs absent à Saint-Romain-en-Gal.

40. Masque de face. Hauteur : 7,5 $\mathrm{mm}$.

Poinçon de Chrysippss présent à Saint-Romain-en-Gal.

41. Masque de face. Hauteur : $6 \mathrm{~mm}$.

Poinçon de Chrysippss présent à Saint-Romain-en-Gal.

42. Masque de face. Hauteur: $7 \mathrm{~mm}$.

Poinçon de Chrysipprs présent à Saint-Romain-en-Gal.

43. Gorgoneion. Hauteur : 7,5 mm.

Poinçon de Chrysipprs présent à Saint-Romain-en-Gal.

44. Tête féminine de face. Hauteur : $8 \mathrm{~mm}$.

Poinçon de Chrysipprs présent à Saint-Romain-en-Gal.

45. Sol ou Apollon? Buste masculin de profil, tourné vers la gauche. Hauteur : $13 \mathrm{~mm}$. La chevelure forme une couronne sur la tête à moins qu'il ne s'agisse d'une couronne radiée. On peut rapprocher ce poinçon, malheureusement mal imprimé, de la représentation de Sol sur des deniers républicains de la période 43-37 avant J.-C. (Grueber, 1910, III, pl. IVIII, 3 et 4), (fig. 79, 7).

Poinçon de Hilarv's Aco inconnu à Saint-Romain-enGal.

\section{Divinités (pl. 75 et 76$)$}

46. Minerve, tournée vers la gauche. Hauteur : $16 \mathrm{~mm}$. La déesse casquée tend le bras droit. Ln bouclier est appuyé contre sa jambe. Une lance est dressée derrière elle. 
Poinçon de Chrysippvs présent à Saint-Romain-en-Gal.

47. Minerve, tournée vers la droite. Hauteur : $25 \mathrm{~mm}$. La déesse casquée pose le pied sur un bouclier et s'appuie sur une lance.

Poinçon d'Hilarvs (?) inconnu à Saint-Romain-en-Gal.

48. Minerve, tournée vers la droite. Hauteur : $12 \mathrm{~mm}$. La déesse, tenant son bouclier, brandit son bras droit.

Poinçon de Chrysippvs présent à Saint-Romain-en-Gal. Le même poinçon se retrouve sur un gobelet d'Hilarvs encadrant la signature avec le poinçon 46 (Fingerlin, 1986, pl. 21, 319, 6).

49. Victoire ailée tournée vers la gauche, tenant un éventail. Hauteur : $12 \mathrm{~mm}$.

Poinçon de Chrysippvs présent à Saint-Romain-en-Gal.

50. Victoire ailée tournée vers la droite brandissant une couronne associée au trophée 62 . Hauteur : $21 \mathrm{~mm}$.

Poinçon de Chrysippvs présent à Saint-Romain-en-Gal.

51.Victoire ailée tournée vers la gauche. Motif incomplet. Associée au trophée 63.

Poinçon de Chrysippvs présent à Saint-Romain-en-Gal.

52.Victoire? Hauteur : $12 \mathrm{~mm}$. Personnage féminin tourné vers la gauche. La main gauche est relevée vers la chevelure, tandis que la droite tient une couromne.

Poinçon de Chrysippvs que l'on rencontre également à Saint-Romain-en-Gal sur des gobelets de Fidelis.

53. Personnage féminin tourné vers la droite. Hauteur : $13 \mathrm{~mm}$.

Poinçon de Chrysippvs inconnu à Saint-Romain-enGal.

54. Vénus au miroir. Hauteur : $14 \mathrm{~mm}$. La déesse tournée vers la gauche est adossée à une colonne et tient un miroir dans sa main.

Poinçon de Chrysippvs présent à Saint-Romain-en-Gal.

55. Fortuna. Hauteur : $15 \mathrm{~mm}$. La déesse tournée vers la gauche s'appuie sur un sceptre et tient dans sa main droite une corne d'abondance.

Poinçon de Chrysipprs présent à Saint-Romain-en-Gal.

56. Femme à sa toilette. Hauteur : $12 \mathrm{~mm}$. Une femme accroupie tournée vers la gauche tient un récipient à la main.

Poinçon de Chrysippvs présent à Saint-Romain-en-Gal.
57. Harpocrate. Hauteur incomplète : $34 \mathrm{~mm}$. Le dieu nu est représenté de face tenant une corne d'abondance, son bras droit replié, un doigt devant la bouche.

Poinçon de Chrysippvs présent à Saint-Romain-en-Gal.

58. Éros avec la massue d'Hercule. Hauteur : 14,5 mm. Le dieu ailé, les jambes croisées, lève les bras au-dessus de la tête. Une massue est figurée à côté de lui.

Poinçon de Chrysippvs présent à Saint-Romain-en-Gal.

59. Néréide et monstre marin. Longueur : $19 \mathrm{~mm}$. La néréïde est accrochée à l'encolure d'un cheval marin tourné vers la droite.

Poinçon de Chrysippvs présent à Saint-Romain-en-Gal.

\section{Symboles divers ( $\mathrm{pl} .76)$}

60. Trophée. Hauteur : $9 \mathrm{~mm}$.

Poinçon de Chrysippvs présent à Saint-Romain-en-Gal.

61. Trophée. Hauteur : $15 \mathrm{~mm}$. De part et d'autre, figurent deux captifs.

Poinçon de Chrysippvs présent à Saint-Romain-en-Gal.

62. Trophée incomplet associé à la Victoire (50).

Poinçon de Chrysippvs présent à Saint-Romain-en-Gal.

63. Trophée incomplet associé à la Victoire (51).

Poinçon de Chrysippvs présent à Saint-Romain-en-Cal.

64. Couronne de laurier. Diamètre : $7 \mathrm{~mm}$.

Poinçon de Chrysippvs présent à Saint-Romain-en-Gal.

65. Trident. Hauteur : $9 \mathrm{~mm}$.

Poinçon de Chrysippvs présent à Saint-Romain-en-Gal.

66. Trident. Hauteur : $8 \mathrm{~mm}$.

Poinçon de Chrysippvs inconnu à Saint-Romain-enGal.

67a. Colonne. Hauteur : $22 \mathrm{~mm}$.

Poinçon de Chrysippvs présent à Saint-Romain-en-Gal.

67b. Colonne. Hauteur : $17 \mathrm{~mm}$.

Poinçon de Chrysipprs présent à Saint-Romain-en-Gal.

68a. Demi-colonne. Hauteur : $10 \mathrm{~mm}$.

Poinçon de Chrysippus présent à Saint-Romain-en-Gal.

68b. Demi-colonne avec chapiteau. Hauteur : $8 \mathrm{~mm}$.

Poinçon de Chrysippvs présent à Saint-Romain-en-Gaal.

69. Vires. Hauteur : $7 \mathrm{~mm}$.

Poinçon de Chrysippus présent à Saint-Romain-en-Gal. 


\section{Animaux (pl. 76)}

70. Dauphin nageant vers la gauche. Longueur : $11 \mathrm{~mm}$. Poinçon de Chrysipprss présent à Saint-Romain-en-Gal.

71.Dauphin nageant vers la droite. Longueur : $11 \mathrm{~mm}$. Poinçon de Chrysipprs présent à Saint-Romain-en-Gal.

72. Dauphin nageant vers la gauche. Longueur : $20 \mathrm{~mm}$. Poinçon de Chrysipprss présent à Saint-Romain-en-Gal.

73a. Poisson nageant vers la droite. Longueur : $10 \mathrm{~mm}$. Poinçon de Chrysipprs présent à Saint-Romain-en-Gal.

73b. Poisson proche du précédent, nageant vers la gauche. Longueur : $10 \mathrm{~mm}$.

Poinçon de Chrysippus présent à Saint-Romain-en-Gal.

73c. Poisson nageant vers la droite. Longueur : $12 \mathrm{~mm}$.

Poinçon de Chrysippus.

74. Aigle. Hauteur: $11 \mathrm{~mm}$.

Poinçon de Chrysippus présent à Saint-Romain-en-Gal.

75. Aigle aux ailes déployées tenant le foudre. Largeur : $13 \mathrm{~mm}$.

Poinçon de Chrysippus présent à Saint-Romain-en-Gal.

76. Oiseau tourné vers la droite. Longueur : $10 \mathrm{~mm}$.

Poinçon de Chrysipprss présent à Saint-Romain-en-Gal.

77. Lièvre. Hauteur : 11,5 mm.

Poinçon de Chrysipprs présent à Saint-Romain-en-Gal.

78. Panthère, motif incomplet. Longueur : $31 \mathrm{~mm}$.

Poinçon de Chrysipprs présent à Saint-Romain-en-Gal.

79a. Abeille.

Poinçon de Chrysipprss présent à Saint-Romain-en-Gal.

79b. Abeille. Longueur : 7,5 mm.

Poinçon d'Hilarvs?

79c. Abeille. Longueur : $5 \mathrm{~mm}$.

Poinçon de Chrysipprs.

80. Taureau cornupète tourné vers la droite. Longueur : $8 \mathrm{~mm}$.

Poinçon de Chrysipprs inconnu à Saint-Romain-enGal.

81. Taureau cornupète tourné vers la gauche. Longueur incomplète.

Poinçon de Chrysipprs inconnu à Saint-Romain-enGal.

82. Insecte, scarabée : Longueur : $11 \mathrm{~mm}$.
Poinçon de Chrysipprs inconnu à Saint-Romain-enGal.

83a. Coquille Saint-jacques. $(8,5 \mathrm{~mm})$.

Poinçon de Chrysipposs.

83b. Coquille Saint-Jacques ( $11 \mathrm{~mm}$ ).

Poinçon d'Hilarss Aco.

83c. Coquille Saint-Jacques $(8 \mathrm{~mm})$.

Poinçon d'Hilarvs Aco.

Motifs végétaux (pl. 77)

84. Grande feuille. Longueur : $22 \mathrm{~mm}$.

Poinçon de Chrysippss présent à Saint-Romain-en-Caal.

85. Grappe de raisin. Longueur : $9 \mathrm{~mm}$.

Poinçon de Chrysippus présent à Saint-Romain-en-Gal.

86. Épi de blé. Longueur incomplète : $9 \mathrm{~mm}$.

Poinçon de Chrysippus présent à Saint-Romain-en-Gal.

87. Épi de blé. Longueur : $12 \mathrm{~mm}$, inversé par rapport à 86.

Poinçon de Chrysippus présent à Saint-Romain-en-Gal.

88. Feuille d'acanthe. Hauteur : $8 \mathrm{~mm}$.

Poinçon de Chrysippus présent à Saint-Romain-en-Gal.

89. Feuille lancéolée. Hauteur : $7 \mathrm{~mm}$.

Poinçon de Chrysipprss présent à Saint-Romain-en-Gal.

90. Feuille de lierre. Hauteur : $5 \mathrm{~mm}$.

Poinçon de Chrỵsipprss présent à Saint-Romain-en-Gal.

91. Feuille de lierre. Hauteur : $5 \mathrm{~mm}$.

Poinçon de Philarcurrs inconnu à Saint-Romain-enGal.

92. Feuille de vigne. Hauteur : $5 \mathrm{~mm}$.

Poinçon de Chrysippus présent à Saint-Romain-en-Gal.

93. Feuille nervurée. Hauteur : $6,5 \mathrm{~mm}$.

Poinçon attribuable à Chrysippuș, absent à Saint-

Romain-en-Gal.

94. Feuille nervurée.

Poinçon d'Hilarss Aco. Moule.

Poinçon absent à Saint-Romain-en-Gal.

95a. Feuille bifide. Hauteur : $5 \mathrm{~mm}$.

Poinçon d'Hilarss présent à Saint-Romain-en-Gal.

95b. Feuille bifide. Hauteur : $6 \mathrm{~mm}$.

Poinçon d'Hilarss présent à Saint-Romain-en-Gal. 
96. Feuille bifide. Longueur : $5 \mathrm{~mm}$.

Poinçon de Chrysippvs, présent à Saint-Romain-enGal.

97. Petite feuille bifide.

Poinçon d'Hilarvs présent à Saint-Romain-en-Cal.

98. Motif de feuille stylisée.

Poinçon de Fidelis présent à Saint-Romain-en-Gal.

99a. Petite feuille (de guirlande). Longueur : 2,5 $\mathrm{mm}$.

Poinçon de Chrysippvs présent à Saint-Romain-en-Gal.

99b. Petite feuille (de guirlande). Longueur : $2 \mathrm{~mm}$.

100. Fleuron à six pétales. $6 \mathrm{~mm}$.

Poinçon de Chrysippus.

101. Flcuron à six pétales. $5,5 \mathrm{~mm}$.

Poinçon d'Hilarvs présent à Saint-Romain-en-Cal.

102. Fleuron à six pétales. $6 \mathrm{~mm}$.

Poinçon de Chrysippus présent à Saint-Romain-en-Gal.

103. Fleuron à huit pétales.

Poinçon de Chrysipprs présent à Saint-Romain-en-( $a$ al.

104. Fleuron à huit pétales $(4,5 \mathrm{~mm})$.

Poinçon de Chrysippus présent à Saint-Romain-en-Cal.

105. Petit fleuron à huit pétales $(4 \mathrm{~mm})$.

Poinçon d'Hilarvs.

106. Fleuron à douze pétales $(7 \mathrm{~mm})$.

Poinçon de Philarcurvs inconnu à Saint-Romain-enGal.

107. Motif de feuilles doubles avec tige pointillée.

Poinçon attribuable à Chrysippvs, inconnu à SaintRomain-en-Gal.

108. Grande feuille nervurée. Hauteur : $15 \mathrm{~mm}$.

Poinçon de Chrysipprs absent à Saint-Romain-en-Cal.

109a. Ornement avec crosses. Hauteur: $6 \mathrm{~mm}$.

Poinçon de Chrysippus présent à Saint-Romain-en-Gal.

109b. Ornement proche de $109 \mathrm{a}$. Hauteur : $6 \mathrm{~mm}$.

Poinçon de Philarcvrvs.

110. Ornement en forme de gousse. Hauteur : $8 \mathrm{~mm}$.

Poinçon de Chrysippus absent à Saint-Romain-en-Gal.

111. Motif en forme de bouton de fleur.

Poinçon de Chrysippus connu à Saint-Romain-en-(ial.

112. Ornement en forme de fruit. Hauteur : $7 \mathrm{~mm}$.

Poinçon de Chrysippus absent à Saint-Romain-en-Gal.
Divers (pl. 77)

113. Motif de double crosse.

Poinçon d'Hilarrs.

114. Motif en losanges imbriqués. Hauteur : $11 \mathrm{~mm}$.

Poinçon de Philarcvrvs absent à Saint-Romain-en-Gal.

115. Disque. Diamètre : $4 \mathrm{~mm}$.

Poinçon de Chrysipprs présent à Saint-Romain-en-Gal.

116. Étoile à huit branches.

Poinçon de Chrysippvs absent à Saint-Romain-en-Gal.

117. Croissant de lune. Diamètre : $15 \mathrm{~mm}$.

Poinçon de Chrysippvs absent à Saint-Romain-en-Gal.

118. Palme. Hauteur : $52 \mathrm{~mm}$ (pl. 74).

Poinçon de Chrysippvs inconnu à Saint-Romain-enGal.

\section{CONCLUSION}

La totalité des gobelets recueillis dans « Gobelet I et II ", et les compléments fournis par la fouille de 1975 permettent de restituer quatre-vingt-treize décors, correspondant à cent un moules au moins. On notera l'existence de plusicurs compositions identiques issues de moules différents et le faible nombre de tirages pour chaque moule, phénomènes déjà observés à Saint-Romain-en-Gal.

L'étude des décors montre la prépondérance de Chrysippvs, à la fois dans le nombre des gobelets produits, et dans la variété et la richesse des décors. Celui-ci totalise en effet plus de la moitié des décors différents utilisant cent trois poinçons. En second lieu, arrive le groupe d'Hilarys et Aco dont la production représente trente et un décors mais seulement quatorze poinçons. Les autres décorateurs ou fabricants de moule, Philocrates, Philarcrrvs, T. Cavivs et Fidelis ne représentent, quant à cux, que $11,5 \%$ de la production et n'utilisent que dix moules et dix poinçons (fig. 80).

Un certain nombre de décors se retrouve dans l'atelier d'Aco de Saint-Romain-en-Gal (Desbat, 1985), où les productions de Chrysippvs dominent également. En revanche, les décors de Philocrates, Philarcurvs et T. Cavivs sont absents de la fosse de rebuts de cuisson de Saint-Romain-en-Gal. Sur un total de cent trois poinçons, seuls dix poinçons de ('hrysippvs n'ont pas été recensés à Saint-Romain-en-Gal. Mais sur la totalité des poinçons de la Muette, vingt-sept manquent à Saint-Romain-en-Gal. Cependant, c'est sur les décors que l'écart est le plus 


\begin{tabular}{|l|r|r|r|r|r|}
\hline \multicolumn{1}{|c|}{ signature } & \multirow{2}{*}{ décors } & moules & poinçons & \multicolumn{2}{|c|}{ Saint-Romain } \\
\hline Chrysippvs & 49 & 55 & 103 & 23 & 12 \\
\hline Aco & 2 & 2 & - & 1 & - \\
\hline Hilarvs-Aco & 29 & 32 & 14 & 7 & 3 \\
\hline Philarcvrvs & 5 & & & & \\
\hline $\begin{array}{l}\text { T. Cavivs } \\
\text { Philocrates }\end{array}$ & 1 & 9 & 8 & - & - \\
\hline Fidelis & 1 & 1 & 2 & - & - \\
\hline indéterminés & 5 & 5 & - & - & - \\
\hline total & 95 & 104 & 127 & 31 & 15 \\
\hline
\end{tabular}

Fig. 80. Nombre de moules, de décors et de poinçons attribuables à chaque décorateur, avec le nombre de moules ou de décors de la Muette. attestés à Saint-Romain-en-Gal.

significatif puisqu'un tiers seulement des décors de la Muette se retrouvent dans la fosse de Saint-Romain-enGal (fig. 80). Les différences observées dans les décors entre Lyon et Saint-Romain-en-Gal ne sont sans doute pas un critère suffisant pour distinguer les deux ateliers. Des décors qui n'ont pas été reconnus dans le matériel de Saint-Romain-en-Gal peuvent quand même très bien avoir été produits également sur place. La vision de l'atelier donnée par la fosse de gobelets est forcément très partielle et ne représente sans doute qu'une partie de la production. La preuve en est que les deux moules trouvés à la Muette ont une composition viennoise alors qu'aucun fragment des décors correspondant ne figure dans les gobelets de Saint-Romain-en-Gal. De même les fragments recueillis dans "Gobelet I et II " ne représentent pas forcément la totalité de la production lyonnaise. La découverte d'un fragment avec une glaçure plombifère incuite laisse supposer qu'une production de gobelets à glaçure, qu'on ne soupçonnait pas jusqu'ici, a bien existé à la Muette.

L'étude des décors met en évidence d'autres phénomènes. On notera en premier lieu le peu d'emprunts constatés entre les fabricants de moules. Les copies de poinçon par surmoulage sont limitées à quelques exemples. Mais pour l'essentiel le répertoire est différent pour chaque potier.

On remarque en revanche les liens existant entre Philarcurvs et T. Cavivs. Non seulement un gobelet associe les deux noms dans la signature mais encore la signature de PHILARCVRVS utilise, pour le C, le même poinçon que celui de Cavivs. Ce poinçon comporte un défaut caractéristique, une petite coupure à l'extrémité basse qui avait d'ailleurs fait croire à l'existence d'un point après le $\mathrm{C}$ (d'où la lecture T.C.avivs). On signalera qu'un gobelet de Neuss (Vegas, 1975, pl. 4, 1), avec un décor absent à la Muette, associe ces deux noms dans la signature : (Philar) cvrvs Cavi (vs). Un autre gobelet trouvé à Roanne associe lui aussi les deux noms (Lasfargues et alii, 1970) de même qu'un gobelet d'Amiens (Massy, Molière, 1979) avec un décor également inconnu à la Muette. Enfin, la signature se rencontre sur un gobelet de Xanten (Hanel, 1995) avec un décor lui aussi inconnu à la Muette.

L'autre remarque concerne les moules. Il est fort intéressant de constater que la production de gobelets d'Aco a nécessité l'utilisation de plus d'une centaine de moules. La production en série des vases moulés exige un grand nombre de moules, le temps de séchage nécessaire au démoulage des vases suppose en effet que le potier puisse disposer de suffisamment de moules pour utiliser une deuxième série pendant que la première est au séchage. Sans cela, le potier ne pourrait avoir qu'une production très faible, la plus grande partie de son activité étant consacrée à la fabrication d'autres céramiques.

La grande quantité des moules de Chrysippvs n'est donc pas une surprise si l'on admet que ce potier était spécialisé dans la fabrication de ce type de gobelet. On peut s'étonner en revanche du faible nombre de décors et donc de moules attribuables à Philarcvrvs, T. Cavivs, Philocrates et Fidelis. Il paraît donc quasiment impossible que ces derniers aient constitué des ateliers indépendants et il est plus probable qu'il faille considérer que tous les moules correspondent en fait à un atelier unique.

Les décors de Chrysippvs présentent un caractère original qui les distingue des autres gobelets d'Aco, qu'il s'agisse des productions gauloises ou des productions italiques. Leur originalité réside surtout dans la richesse iconographique et le grand nombre de poinçons utilisés mais également dans les thèmes abordés. Toute la série des gobelets à décor de gladiateurs est très intéressante à plus d'un titre. Ils nous présentent des couples de gladiateurs rarement figurés comme ces combattants aveugles, ou les combats d'archers, mais l'intérêt de ces représentations réside surtout dans le fait qu'elles sont antérieures à la réforme de la gladiature par Auguste. La question est de savoir jusqu'à quel point ces représentations sont réalistes et peuvent fournir des indications sur l'armement des gladiateurs. L'identification des gladiateurs n'est pas 
évidente, hormis celle du rétiaire, mais on croît pouvoir reconnaitre la figuration d'un mirmillon dans le gladiateur portant sur le casque un cimier d'un type particulier (cf. décors 1, 2, etc.). De même le gladiateur au grand bouclier affronté au rétiaire pourrait être le Gaulois qui lui était traditionnellement opposé et dont les représentations disparaissent après Auguste.

Les autres gobelets dont l'iconographie appelle une remarque sont ceux où apparaissent les bustes d'Auguste et peut-être d'Agrippa. Ces gobelets associent d'autres symboles : dauphins, tridents, divinités marines, épis de blé, mais aussi Victoires, trophées et couronnes. De telles associations ne peuvent être le fait du hasard. La présence sur certains gobelets du dieu Harpocrate associé à des Victoires portant des trophées (décor 37) renforce l'aspect symbolique de ces décors, et invite à y voir des références à la bataille d'Actium et à la conquête de l'Égypte en même temps qu'une glorification de la famille impériale. De même, la figuration d'un buste radié, dans lequel on peut reconnaitre Sol ou Apollon, peut être interprétée comme une référence à la symbolique augustéenne.

Du point de vue chronologique, les gobelets d'Aco ne se rencontrent que dans la phase ancienne de production de l'atelier. Leur association avec les vases de sigillée permet de placer la date de production des gobelets de "Gobelet I et II » entre 15 et 10 avant J.-C. Leur rareté dans le matéricl de Haltern et leur faible représentation dans celui d'Oberaden sont un argument pour valider cette datation. On note en revanche une plus grande représentation des gobelets d'Aco dans le camp de Dangstetten (Fingerlin, 1986), où l'on retrouve des productions de Chrysippvs, Philarcvrvs, de T. Cavivs et d'Hilarvs, qui représentent $24 \%$ des céramiques et céramiques à parois fines. Il n'est pas certain toutefois qu'il s'agisse exclusivement de gobelets de la Muette, une origine viennoise étant également possible. Tous les éléments concourent à placer la production des gobelets d'Aco à la Muette sur un temps relativement court, et à la situer avant la grande période de diffusion de l'atelier.

L’iconographie des gobelets de Chrysippvs fournit un terminus post quem pour sa production. Nous arons vu que certains décors offrent une symbolique qui se rattache à la victoire d'Actium et constitue une glorification d'Octave Auguste. Le portrait que l'on peut identifier sans conteste avec celui d'Octave présente des analogies certaines avec les frappes réalisées en 29 avant J.-C. dans l'atelier de Rome notamment (Giard, 1976, pl. I, n² 20 sqq.) (cf. fig. 79, 1-4). D’autres poinçons offrent également des rapprochements avec les séries monétaires. La comparaison avec le monnayage d'Octave permet d'ailleurs d'interpréter le buste féminin associé à celui d'Octave sur plusieurs gobelets comme un buste de Vénus et non celui de Livie, ainsi qu'on avait pu l'identifier (Vierneisel, Zanker, 1979; Desbat, 1990a). Ce monnayage montre en effet une représentation qui sans être identique est très voisine : Vénus au diadème, avec un collier (Giard, 1976, pl. I, 1 à 11) (cf. fig. 79, 5). On remarquera que cette association n'est pas fortuite, Vénus étant la déesse de la Gens Julia. On peut noter que d'autres symboles figurés sur les gobelets, Victoire, trophées, épis de blé, Fortune, se retrouvent sur le monnayage de l'époque. De même le taureau cornupète, présent dans le décor 29 de Chrysipprs, est un thème que l'on retrouve sur les monnaies d'Auguste, en particulier sur le monnayage de Lyon entre 16 et 12 avant J.-C. (Grueber, 1910, III, pl. (XVII).

L'identification du troisième buste (poinçon 28) pose plus de problèmes. La comparaison avec les as de Nîmes (qui d'ailleurs se réfèrent à la conquête de l'Égypte) permet de penser qu'il s'agit d'Agrippa (cf. fig. 79, 6). La chronologie traditionnellement proposée, qui situe la première série à partir de 28 avant J.-C., serait en concordance avec le terminus fourni par les autres portraits. Toutefois, cette datation a été remise en cause par $M$. Christol et Chr. Goudineau (1988, p. 101-102) pour qui l'association d'Agrippa et d'Auguste au droit d'une monnaie traduirait une co-régence qui ne peut être antérieure à 18 avant J.-C. Ils rejoignent ici l'opinion de A. Chastagnol (1966) : " pour que les effigies d'Auguste et d'Agrippa soient disposées côte à côte sur un plan d'égalité, mais avec la titulature propre au seul Auguste, il faut qu'Agrippa ait été associé officiellement aux pouvoirs de son ami, notamment à la puissance tribunicienne, ce qui advint seulement en 18 avant J.-C., alors qu'il était devenu depuis trois ans le gendre du prince par son mariage avec la jeune Julie. ". Si l'identification d'Agrippa sur les gobelets était assurée, il faudrait donc placer la fabrication des gobelets après 18 , à moins d'envisager que la représentation d'Agrippa ne soit pas liée au monnayage de Nîmes mais ait une autre source. On rappellera que sur une monnaie de Simope frappée en 27, figurent Auguste au droit et Agrippa au revers. Pour J.-M. Roddaz (1984), l'association d'Auguste et Agrippa sur le monnayage de Nîmes peut bien être antérieure à la co-régence de $18 \mathrm{et}$ correspondre à la volonté d'Auguste d'associer Agrippa, 
avec lequel il partage théoriquement en 28-27 avant J-C. l'égalité des pouvoirs". Quoiqu'il en soit la chronologie des productions de Chrysippvs ne peut donc être au plus tôt que postérieure à 29 avant J.-C.., et pourrait plutôt se situer après 18 avant J.-C. Cette datation basse serait tout à fait concordante avec celle proposée pour le fonctionnement de la Muette et le début de la première phase. De plus, elle se situerait à une époque qui suit de près le séjour d'Agrippa en Caule, en 20-19 avant J.-C. et peut aussi correspondre à la venue d'Auguste en 16 avant J.-C.

A. D.

\section{LES MOULES}

Dix-sept moules ou fragments de moules ont été recueillis sur le site de la Muette dans des contextes différents. La majorité d'entre eux a été trouvée en 1966, seuls deux fragments proviennent du sondage de 1975. La plupart appartient à des contextes de la phase 2.

Un autre moule de sigillée (8) a été trouvé en dehors de la Muette, sur le site de Saint-Vincent, dans un remblai postérieur au fonctionnement de l'atelier. On ignore s'il provient de cet atelier ou s'il s'agit d'un élément déplacé originaire d'un autre site de production. Il nous a semblé préférable en tout cas de le publier avec les moules du même type trouvés à la Muette.

Tous ces fragments peuvent être classés en trois catégories.

\section{LES MOULES DE SIGILLÉE (pl. 78, 1-11)}

Au nombre de douze exemplaires, les moules utilisés pour le façonnage de la sigillée sont aisément identifiables, ils appartiennent à trois types.

\section{TYPE 1}

Huit fragments correspondent à des moules hémisphériques destinés à la fabrication de vases en forme de

\footnotetext{
8. J.-M. Roddaz (1984. p. 206-207) considere qu une grande publicité a été donnée dans tout l'Empire à cette association et que leur présence sur les as de Nîmes " commémore, au-delà de la victoire d'Actium et du succès sur l'Égypte, le double consulat de $28-27$ alant J.-C. et traduit la volonté d'Octavien-Auguste de placer son collègue sur le même plan que lui ".
}

calice, de tvpe Dragendorff 11 (Conspectus R8 ou R9). Quatre présentent un rebord avec une lère triangulaire débordante, que l'on retrouve sur certains moules arétins (Brown, 1968, XIX, 62, 77). Un autre fragment appartient à un fond avec un pied annulaire (4). Les deux derniers exemplaires sont des fragments de la panse d'un moule (pl. 79, 5 et pl. 80, 7$)^{9}$.

\section{Moule 1a (pl. 78 et 79)}

Fragment de moule hémisphérique avec rebord, diamètre restitué extérieur : $20,2 \mathrm{~cm}$, diamètre intérieur : $17 \mathrm{~cm}$.

Origine : Muette, 1966, analyse MML 119.

Le décor se compose d'une rangée d'oves, réalisée non pas avec une roulette mais arec un poinçon reporté plusieurs fois. Cet ove est très proche sinon identique à l'ove 1 de H. Dragendorff et C. Watzinger (1948, fig. 1, 1), attribué à Perennius Tigranvs (Oxé, 1968, pl. XXVII$\mathrm{XXX)}$. Sous la rangée d'oves apparaît un guerrier nu, casqué, se retournant vers l'arrière. Il est à demi agenouillé, sa jambe gauche repliée sous lui. Son bras droit tendu autour duquel s'enroule une draperie paraît tenir un bouclier. Son bras gauche se termine par un poing serré, comme s'il tenait une arme, non figurée. Dans le champ se dresse un bouquet d'acanthe.

\section{Moule 1b (pl. 78 et 79)}

Fragment de moule hémisphérique avec rebord appartenant au même moule, diamètre restitué : comme le précédent.

Origine: Muette, 1975, analyse MML 181.

Le décor se compose de la même rangée d'ores. Audessous apparaît le sommet d'une feuille d'acanthe et à droite, un motif incomplet, non identifiable.

\section{Moule 2 (pl. 78 et 79 )}

Fragment de moule hémisphérique avec rebord, diamètre extérieur restitué : $24 \mathrm{~cm}$.

Origine : Muette, 1966, analyse MMI. 116.

Le décor se compose de la même rangée d'ores que sur le moule 1 et d'une feuille d'acanthe vue de face.

9. Les décors sont décrits en positif. Le's dessins des poinçons ont cete réatlisés à partir de moulages en argile. Ies dimensions sont dommées daapres les dessins qui tiennent compte du retrait de l'argile. 
Moule 3 (pl. 78 et 79)

Fragment de moule hémisphérique avec rebord, diamètre indéterminé.

Origine : Muette, 1966, analyse MML 120.

Le décor se compose de la même rangée d'oves que sur les trois moules précédents. En dessous, apparaît la tête d'un personnage barbu, tendant le bras en direction d'un oiseau.

\section{Moule 4 (pl. 78 et 79 )}

Fragment de fond de moule hémisphérique avec pied annulaire.

Origine : Muette, 1966, analyse MML 112.

Le décor montre à la base une frise de feuilles surmontée par une feuille d'acanthe vue de face, d'où sortent deux ornements torsadés. Des rinceaux se déploient de part et d'autre de la base de la feuille.

Moule 5 (pl. 79)

Fragment de panse de moule hémisphérique.

Origine : Muette, 1966, analyse MML 121.

Décor de feuille d'acanthe, peut-être le même que sur le moule 2.

Moule 6 (pl. 78 et 80 )

Fragment de moule hémisphérique avec rebord, diamètre indéterminé.

Origine : Muette, 1966, analyse MML 93.

Le décor se compose d'une frise d'oves avec lancettes, surmontée d'un rang de perles. En dessous apparaît le sommet d'une palmette. La frise d'oves avec rang de perles est très proche de celle des moules de $M$. Perennius Bargathes (Bartoli et alii, 1984, moules 96 et 97 , et pl. II, ove 4). La palmette semble elle aussi identique à un poinçon de Bargathes (Bartoli et alii, 1984, p. 123 et pl. II, $\left.n^{\circ} 9\right)$.

Moule 7 (pl. 80)

Fragment de panse de moule hémisphérique.

Origine : Muette, 1975, analyse MML 200.

Le décor montre la partie inférieure d'un personnage debout, vêtu d'une tunique.
Moule 8 (pl. 78 et 80 )

Fragment de moule hémisphérique avec rebord, diamètre extérieur : $20 \mathrm{~cm}$, diamètre intérieur : $16,6 \mathrm{~cm}$.

Origine : Saint-Vincent, 1985. Analyse, MML 243.

Le décor, limité au sommet par une frise de feuilles se chevauchant horizontalement, se compose de deux groupes de poinçons identiques associant une palmette au-dessus d'une feuille dentelée. De la base de la palmette et de la feuille, partent des pointes torsadées obliques disposées symétriquement. À la base un rang de perles limite le décor.

Comme pour le moule 6 , on relève des analogies avec la production de Bargathes : une frise avec feuilles semblables disposées verticalement existe sur un moule (Bartoli et alii, 1984, pl. II, 17) de même que les motifs torsadés (Bartoli et alii, 1984, moule 19, p. 46).

Le motif de feuille se retrouve en outre sur un des vases de Haltern (Rudnick, 1994, pl. 47, n ${ }^{\circ}$ 169) avec un poinçon identique, pour lequel une origine lyonnaise était envisagée (Rudnick, 1994, p. 214, n 169).

\section{TYPE 2}

Le deuxième type de moule est représenté par deux fragments de forme aplatie, dont le rebord est conservé. La faible profondeur de ces moules permet de penser qu'ils ont été utilisés pour décorer la partie basse des calices carénés, réalisés à l'aide de moules en plusieurs parties. De tels calices sont connus par quelques exemples - cf. un calice à Haltern (Rudnick, 1994, forme 7a) et d'Asciburgium (fig. 81) - et se retrouvent également à une date plus tardive à la Graufesenque.

Moule 9 (pl. 78 et 80 )

Fragment de moule avec rebord, diamètre restitué : $16 \mathrm{~cm}$.

Origine : Muette, 1966, analyse MML 115.

Le décor se compose d'une rangée de feuilles d'acanthe, alternant avec un motif fuselé, torsadé. Le bouquet de feuilles d'acanthe est réalisé avec le même poinçon que dans le moule 1 . De même, le motif torsadé semble correspondre au même poinçon que celui utilisé dans le moule 5 . 


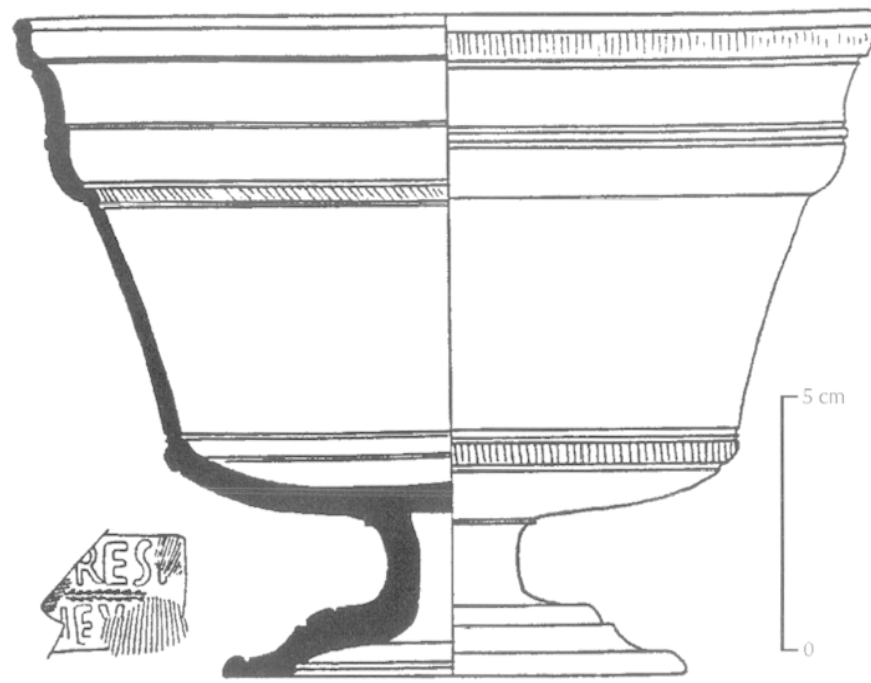

Fig. 81. Calice d'Asciburgium (d'après Bechert, Landerhorven, 1968).

Moule 10 (pl. 78 et 80)

Fragment de moule avec rebord, diamètre restitué : $17,2 \mathrm{~cm}$.

Origine : Muette, 1966, analyse MML 113.

La lèvre de profil triangulaire présente deux sillons internes. Un léger décrochement marque la base. Le décor se compose d'une frise de doubles cercles concentriques entre deux sillons. En dessous apparait un gladiateur incomplet reconnaissable à son casque et à son bouclier rond. Un autre personnage incomplet, dont il ne subsiste qu'une jambe et un bras, lui fait face. Il s'agit sans doute d'un autre gladiateur. Entre les deux, figure un masque.

\section{TYPE 3}

Le dernier type est représenté par un unique moule de petite taille, de forme hémisphérique à lèrre triangulaire. Sa taille exclut qu'il s'agisse d'un vase caliciforme, mais il pourrait plutôt correspondre à une petite coupe. C'est le seul moule qui livre une signature intradécorative.

Moule 11 (pl. 78 et 80)

Fragment de moule hémisphérique avec rebord, diamètre extérieur : $10,4 \mathrm{~cm}$, diamètre intérieur : $4,8 \mathrm{~cm}$.

Origine : Muette, 1966, analyse MML 92.
Le décor se compose d'une frise de cercles pointés, surmontant des palmettes. Dans le décor apparait l'estampille HERACLI (DA)/PVBLI (O.C. 1430-1431). L 'analyse de ce moule arait conclu à une origine italique, arétine (Picon, Lasfargues, 1974).

\section{Catalogue des poinçons de sigillée (figg. 82)}

1. Ove de Perennius Tigranss? Hauteur: $11 \mathrm{~mm}$, moules 1,2 et 3 (pl. 79);

2. Ove de Perennius Bargathes? Hauteur : $10 \mathrm{~mm}$, moule 6 (pl. 80);

3. Motif de feuille, frise du moule de Saint-Vincent, longueur : $7 \mathrm{~mm}$, moule 8 (pl. 80);

4. Motif de feuille dentelée, frise inférieure du moule, longueur : $8 \mathrm{~mm}$, moule 4 (pl. 79) ;

5. Feuille, hauteur : $21 \mathrm{~mm}$, moule 8 (pl. 80);

6. Palmette, hauteur conservée : $19 \mathrm{~mm}$, moule 8 (pl. 80) ;

7. Motif torsadé, hauteur : $31 \mathrm{~mm}$, moule 8 (pl. 80);

8. Feuille d'acanthe, hauteur : $41 \mathrm{~mm}$, moule 9 (pl. 80$)$.

\section{LES MOULES DE GOBELETS (pl. 78, 80 et 81)}

Un exemplaire complet et trois fragments appartiennent à des moules hémisphériques de petite taille.
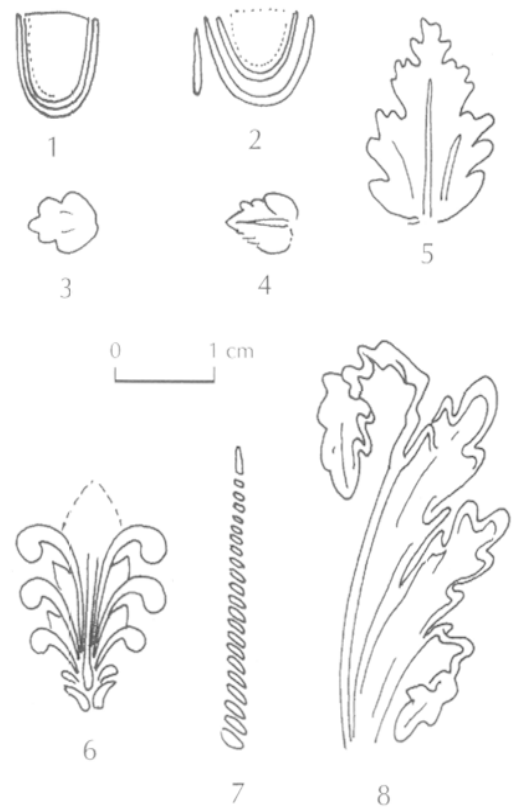

Fig. 82. Poincons des moules de sigillee. 
Aucune production correspondant à ces moules n'a été identifiée parmi l'abondant matériel récolté, et l'on ignore quel type de céramique a été précisément fabriqué avec ces moules : céramique à parois fines à pâte siliceuse, gobelet en sigillće ou à glaçure plombifère? Il est impossible d'acquérir une certitude. On connaît des vases à parois fines de forme globulaire avec un décor de lignes, de perles ou de clous, qui pourraient avoir été façonnés au moule. De tels vases sont présents à Haltern avec glaçure plombifère (Loeschcke, 1909, type 23 et 43), ainsi qu'à Saint-Romain-en-Gal (Desbat et alii, 1994; Desbat, 1996), avec ou sans glaçure. Toutefois, le décor de ces vases diffère de ceux des moules de la Muette, qui présentent un décor couvrant.

Moule 12 (pl. 78 et 80 )

Moule complet, de forme hémisphérique, à lèvre triangulaire et pied annulaire. Hauteur $: 6,1 \mathrm{~cm}$, diamètre extérieur : $10,6 \mathrm{~cm}$, diamètre intérieur : $8,6 \mathrm{~cm}$.

Origine : Muette, 1966, analyse MML 114.

Le décor est composé de trous juxtaposés, formant des triangles, disposés en quinconce, séparés par des bandes lisses de $5 \mathrm{~mm}$ de large.

Moule 13 (pl. 78 et 81 )

Fragment de moule avec lèvre en triangle, diamètre restitué : $12,2 \mathrm{~cm}$.

Origine : Muette, 1966, analyse MML 122.

Le décor est constitué de trames.

Moule 14 (pl. 78 et 81)

Fragment de moule avec lèrre en triangle, diamètre restitué : $10,4 \mathrm{~cm}$.

Origine : Muette, 1966, analyse MML 123.

Le décor se compose de lignes de trous irréguliers.

Moule 15 (pl. 78 et 81)

Fragment de panse.

Origine : Muette, 1966, pas analysé.

Le décor se compose de trous comme dans les exemplaires précédents, plusieurs gorges soulignent l'ensemble à la base.

\section{LES MOULES DE GOBELETS D'ACO}

Les deux derniers moules correspondent à la fabrication des gobelets d'Aco. Il s'agit de moules de forme légèrement tronconique. Le premier n'est représenté que par un fragment, le second presque complet comprend six fragments. Seule la partie supérieure des moules est conservée. Elle présente une lèvre arrondie sans rebord. On ignore comment se terminait la base mais on peut penser que le fond était ouvert, comme c'est le cas pour certains gobelets de Lezoux. Compte tenu de l'étroitesse des moules, l'absence de fond est un élément qui facilitait la réalisation de décor, lors de la fabrication du moule.

Moule 16 (pl. 78 et 81 )

Moule de gobelet de Chrysippvs constitué de six fragments. La circonférence est complète, mais le fond manque, diamètre extérieur : $9,6 \mathrm{~cm}$, diamètre intérieur : $8 \mathrm{~cm}$.

Origine : Muette, 1966, M.T.S., analyse MML 117.

Le décor se développe sous une frise de feuilles. Deux paires de gladiateurs s'affrontent, séparés par un laniste brandissant une canne. Au-dessus du décor, sous la frise, la signature de CHRYSIPPVS s'inscrit entre deux étoiles à huit branches (cf. supra, étude des décors de gobelets de Chrysippss). Ces fragments sont le seul exemple de moule de Chrysippvs.

Moule 17 (pl. 78 et 81 )

Fragment de rebord de moule de gobelet d'Hilarvs Aco, diamc̀tre extérieur : $11 \mathrm{~cm}$, diamètre intérieur : $9,8 \mathrm{~cm}$.

Origine : Muette, 1966, M.T.S., analyse MML 118.

Le décor se compose d'un semis de picots surmonté d'une frise de feuilles. Sous la frise s'inscrit la signature d'(HILAR) VS. ACO encadrée par une feuille. Cette frise se retrouve sur d'autres gobelets d'Hilarvs Aco (cf. supra), mais avec un motif d'encadrement de la signature, différent.

\section{UN POINÇON MATRICE}

Le dernier élément lié à la fabrication des moules est un poinçon matrice découvert en 1975 (pl. 80, 18). La face où figurait le motif est malheureusement endommagée. Sa longueur est de $49 \mathrm{~mm}$, la hauteur conservée sur 
la face figurée est de $38 \mathrm{~mm}$. Ia pâte roséc est recouverte d'un vernis rouge identique à celui des sigillées. Malgré sa dégradation, on devine un motif représentant un personnage debout, le bras droit écarté du corps. On peut penser à un gladiateur, que l'on peut rapprocher d'un des deux personnages figurés sur le moule 10 . La forme de la jambe, ainsi que la position du bras sont identiques. Ia composition de ce poinçon exclut une origine arétine mais suggère une origine viennoise.
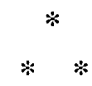

Tous les fragments de moules ainsi que les poinçons recueillis sur l'atelier se caractérisent par des pâtes fines, de couleur beige ou beige rosé. On peut remarquer toutefois quelques différences. Trois exemplaires $(7,8$ et 11$)$ présentent un épiderme interne très lisse et régulier, à la différence des autres exemplaires dont la surface est tendre, rayée et irrégulière. L.es trois exemplaires cités présentent en outre une pâte de couleur homogène, rosée, alors que tous les autres montrent une différence de coloration entre la surface extérieure de couleur beige et la pâte rosée.

Presque tous les fragments ont fait l'objet d'analyses physico-chimiques. Les analyses effectuées sur les exemplaires recueillis en 1966 avaient démontré l'existence de deux moules importés d'Arezo (Picon, Lasfargues, 1974). Il s'agit des moules 7 et 11 . Les compléments réalisés sur les moules trouvés en 1975 ont révélé l'existence d'un troisième moule arétin (8). Il est intéressant de constater qu'il s'agit des fragments qui présentaient une légère différence d'aspect avec les autres moules, et en particulier du moule estampillé HERACLIDA/PVBLI (11).

I a poursuite des recherches sur les argiles régionales et les progrès réalisés dans la séparation entre les argiles de Lyon et celles de Vienne permettent aujourd'hui de proposer une origine viennoise pour plusieurs des moules utilisés à la Muette, en particulier les deux moules de gobelets d'Aco.

Il faut rappeler qu'aucun fragment de vase n'a pu être mis en relation avec les moules, qu'il s'agisse des moules de sigillée ou des moules de gobelets. Ce seul fait montre bien que notre comnaissance de l'atelier de la Muette et de ses productions est loin d'être exhaustive. La découverte d'un autre moule de sigillée en dehors du site de la Muette ra dans le même sens et confirme que cet atelier ne représente qu'une partie d'un vaste complexe de potiers augustéens.

Il faut noter encore que plusieurs des moules trourés à Lyon ont montré des compositions viemnoises qui attestent qu'ils ont èté fabriqués à Vienne et probablement utilisés sur place, arant leur transfert à Lyon.

Concernant les moules de sigillée, on notera que la finesse des détails mais surtout la taille des motifs (en particulier les ores) semblent indiquer que les moules lyonnais ou viennois ont été réalisés, au moins en partie, avec des poinçons originaux et non à l'aide de poinçons obtenus par surmoulage à partir de vases arétins ou d'autres moules. Si tel est bien le cas, il en découle que l'atelier de la Muette et probablement avant lui un atelier viennois ont sinon accueilli des potiers du cercle de Perennius, au moins utilisé des poinçons et des moules qui provenaient de cette officine.

A. D.

\section{LA CÉRAMIQUE À ENGOBE INTERNE ROUGE POMPÉIEN}

Ce type de céramique fait partie des "céramiques communes culinaires" qui résistent au feu. Cet usage culinaire est clairement attesté par la morphologie des plats (Bats, 1988, p. 69), leur association à un couvercle, et la présence sur les plats d'un engobe interne. Leur usage a pu être précisé grâce à la découverte à Pompéi de certains de ces plats qui contenaient encore des galettes de pâte à pain (Loeschcke, 1909, p. 268; Goudineau, 1970 , p. 165). Ils sont donc destinés à l'enfournement et sont conçus de façon à éviter que le contenu n'attache au récipient, d'où la présence utilitaire d'un engobe (on peut penser que s'il avait eu une fonction décorative, l'engobe serait présent aussi sur la paroi externe). Cet engobe peut être appliqué sur la lèvre jusqu'au bord, voire jusqu’à $2 \mathrm{~cm}$ au plus bas du côté extérieur.

Les plats sont généralement de grande dimension (30) à $40 \mathrm{~cm}$, voire $50 \mathrm{~cm}$ de diamètre), bien que les diamètres n'excèdent pas $30 \mathrm{~cm}$ dans l'atelier de la Muette. Leurs bases, le plus souvent concaves, portent parfois un pied matérialisé par un anneau et leurs fonds internes sont, le plus sourent, dotés d'une à trois zones de stries concentriques.

Le couvercle n'est jamais engobé et reste dépourvu d'élément de préhension, mais il présente généralement 
un anneau en relief, qui peut servir de pied annulaire lorsqu'on l'utilise comme assiette pour démouler la " galette ". L'association du plat avec un couvercle, semble-t-il pour faciliter le démoulage, l'absence d'élément de préhension et l'engobage intérieur mettent en évidence la fonction de moule du récipient, destiné à être enfourné. Cette fonction spécifique était connue au moment de la production.

\section{CARACTÉRISTIQUES TECHNIQUES}

S'agissant de céramique culinaire, l'argile utilisée comporte généralement des dégraissants ajoutés et un important taux de silice (Batigne, 1995) ; la pâte est de teinte à dominante rouge, car le taux de fer qu'elle contient est élevé et la post-cuisson s'effectue en atmosphère oxydante. Ce type de production devait être cuit à basse température afin de bien conserver l'engobe et de résister aux chocs thermiques par la suite (Picon, Vichy, 1974, p. 51). D'ailleurs, la terminologie parfois employée pour désigner ce type de céramique - VRP : vernis rouge pompéien - n'est pas très précise puisqu'il s'agit d'un engobe, c'est-à-dire d'un vernis argileux non grésé (aspect mat et porosité), sa facture (notamment la couleur, l'épaisseur et l'aspect brillant) étant intentionnelle. La technique de fabrication répond à des procédés simples : un engobe non grésé se réoxyde rapidement lors de la post-cuisson, il doit être cuit à basse température pour ne pas se craqueler même s'il est épais, et cette épaisseur permet un lissage qui se rapproche de la brillance qui résulte du grésage (Goudineau, 1970, p. 164-165). Cette fabrication ne nécessite ni technique, ni structure de cuisson spécifique comme c'est le cas pour la sigillée.

En ce qui concerne précisément les productions de l'atelier de la Muette, deux argiles non calcaires ont été utilisées (Picon, Vichy, 1974, p. 41) "1", l'une à pâte fine, l'autre à pâte grossière : soit les potiers avaient deux sources d'approvisionnement en argiles distinctes, soit l'une des pâtes comporte un dégraissant ajouté (grains de quartz à granulométrie variable). La structure de la pâte, qu'elle soit fine ou grossière, est feuilletée et la matrice argileuse comporte de nombreuses particules de mica blanc. L'aspect de ces productions est très proche de celui des productions de Campanie mais sans les minéraux volcaniques noirs (pyroxènes).

10. Argile 6 a $65,58 \%$ de $\mathrm{Si} \mathrm{O}$, et argile 7 à $62.23 \%$ de Si O..
Le lot comprend majoritairement des surcuits (rebuts de cuisson issus de dépotoirs) de couleur foncée et parfois déformés.

\section{DONNÉES NUMÉRIQUES}

L'étude quantitative se fonde sur le matériel exhumé lors des découvertes de 1966 et 1975; cet échantillon est faiblement représenté par rapport à l'essentiel de la production. On compte au total 1526 fragments (tabl. VII).

L'ensemble II est moins bien représenté. La répartition plats et couvercles, relativement déséquilibrée, n'est pas significative dans le cadre d'une étude du matériel issu de dépotoirs qui n'ont pas été appréhendés de façon exhaustive.

Pour l'inventaire, on a procédé de la même façon que pour les productions principales (sigillée et céramique à parois fines) : après tri des bords et des fonds principalement (les fragments de panse sont rares et le matériel est peu fragmenté en raison de la résistance des pâtes à caractère siliceux), une répartition des plats et des couvercles, puis un classement des formes ont pu être effectués. Les comptages présentent donc le nombre de bords (seul critère discriminant pour ce type de production) afin de déterminer les différents types et la représentation de chacun d'cux en pourcentage (tabl. VII et VIII).

\section{TYPOLOGIE (pl. 82)}

L'ordre adopté pour le classement typologique reprend l'ordre décroissant de la fréquence des formes dans les deux contextes chronologiques, nous espérons ainsi pouvoir refléter une évolution chronologique de la production en relation avec son évolution morphologique.

La définition des types de plats a été effectuée selon l'orientation de la paroi et la morphologie de la lèvre.

Tabl. VII. Céramique à engobe interne rouge pompéien de l'atelier de la Muette : répartition en nombre de bords des céramiques dans les differents contextes.

\begin{tabular}{|l|r|r|r|r|r|r|r|r|r|}
\hline \multirow{2}{*}{$\begin{array}{l}\text { nombre total } \\
\text { de fragments }\end{array}$} & \multicolumn{4}{|c|}{ ensemble I } & \multicolumn{4}{c|}{ ensemble II } & \multirow{2}{*}{ total } \\
\cline { 2 - 11 } & Gob.I & Gob.II & M.U.75 & total & T.N.E. & M.T.S. & S3/S4 & total & \\
\hline plats & 193 & 136 & 132 & 461 & 171 & 39 & 70 & 280 & 741 \\
\hline couvercles & 364 & 187 & 114 & 665 & 70 & 11 & 39 & 120 & 785 \\
\hline total & 557 & 323 & 246 & 1126 & 241 & 50 & 109 & 400 & 1526 \\
\hline
\end{tabular}


Tabl. VIII. Céramique à engobe interne rouge pompëien de l'atelier de la Muette : quantification de chaque type en pourcentage dans les deux ensembles.

\begin{tabular}{|l|r|r|r|r|r|r|}
\hline $\begin{array}{c}\text { fragments } \\
\text { de bords }\end{array}$ & \multicolumn{2}{|c|}{ ensemble I } & \multicolumn{2}{|c|}{ ensemble II } & \multicolumn{2}{|c|}{ total } \\
\hline types & nombre & $\%$ & nombre & $\%$ & nombre & $\%$ \\
\hline $1 \mathrm{~A}$ & 136 & 26,1 & 5 & 3,4 & 141 & $\mathbf{2 1 , 1}$ \\
\hline $1 \mathrm{~B}$ & 45 & 8,6 & 11 & 7,5 & 56 & $\mathbf{8 , 4}$ \\
\hline $1 \mathrm{C}$ & 11 & 2,1 & 8 & 5,5 & 19 & 2,9 \\
\hline total 1 & 192 & 36,8 & 24 & $\mathbf{1 6 , 4}$ & 216 & 32,4 \\
\hline 2 & 2 & 0,4 & 45 & 30,8 & 47 & 7 \\
\hline 3 & 5 & 1 & 14 & 9,6 & 19 & 2,9 \\
\hline 4 & 1 & 0,2 & 2 & 1,4 & 3 & $\mathbf{0 , 4}$ \\
\hline 5 & 186 & 35,7 & 8 & 5,5 & 194 & $\mathbf{2 9}$ \\
\hline 6 & 103 & 19,8 & 20 & 13,7 & 123 & $\mathbf{1 8 , 5}$ \\
\hline 7 & 32 & 6,1 & 33 & 22,6 & 65 & 9,8 \\
\hline total & 521 & $\mathbf{1 0 0}$ & 146 & $\mathbf{1 0 0}$ & $\mathbf{6 6 7}$ & $\mathbf{1 0 0}$ \\
\hline
\end{tabular}

\section{Paroi CONCAVE}

Type 1 : bord épaissi en triangle, rentrant, dont trois variantes ont été déterminées selon son orientation :

- variante $1 \mathrm{~A}$ : oblique vers l'extérieur,

- variante $1 \mathrm{~B}$ : oblique vers l'intérieur,

- variante $1 \mathrm{C}$ : droit.

Type 2 : bord non épaissi, simple, arrondi.

\section{PAROI CONVEXE}

Type 3 : bord évasé à bourrelet externe, forme évolutive non stabilisée. Diverses formes de bourrelet ont été repérées au cours de l'étude; elles constituent des variantes dans le classement en raison de la faiblesse de l'échantillon (un seul individu représente chaque variante virtuelle de cette forme).

Type 4 : bord saillant; il pourrait s'agir soit de l'amorce d'une nouvelle production, soit d'éléments résiduels, soit d'éléments extérieurs à l'atelier, puisqu'on a comptabilisé seulement deux individus.

\section{COUVERCLE}

Type 5 : bord simple, pied annulaire attesté.
Type 6: bord triangulaire, pied annulaire attesté.

Type 7 : bord à rebord, pied annulaire non attesté.

Ces trois formes sont assez proches. Le type 6, qui présente un replat sur la lèrre externe, n'est qu'un intermédiaire entre la forme simple et la forme qui résulte de l'accentuation du replat. Ce caractère de la lèvre ménage une surface plus importante en contact avec le plat, phénomène semblant lié à des considérations pratiques.

\section{Type 1}

Cette forme à paroi concave et bord aplati orienté vers l'extérieur (A), vers l'intérieur (B) ou droit (C) est la plus représentéc et largement majoritaire dans l'ensemble I. Les variantes déterminées selon l'orientation du bord semblent être davantage tributaires du tour de main du potier que d'une évolution morphologique liée à une nécessité fonctionnelle, surtout sur une période aussi courte. La variante $1 \mathrm{~A}$, forme de plat essentielle de l'ensemble I d'après la répartition (26,1\% des bords de la totalité du lot), semble concurrencée par les variantes $1 \mathrm{~B}$ (bord incliné vers l'intérieur) et $\mathrm{IC}$ (bord droit) dans l'ensemble II.

La gorge externe n'est pas systématique mais son absence est exceptionnelle sur les bords appartenant à ce type.

Les quelques données que nous avons recueillies indiquent que l'apparition de ce type varie d'une région à l'autre. Le bord de la variante A est attesté dans les niveaux datés de 40 à 10 avant J.-C. à Bolsena (Goudineau, 1970, pl. VIII, n 5 , couche 2c), et à Genève à l'époque gallo-romaine précoce (Paunier, 1981, p. 259 et $\left.368, \mathrm{n}^{\circ} 589\right)^{11}$ mais sur paroi oblique rectiligne. On peut signaler en outre la présence de cette même forme à Niederbieber à la fin du II" s. (Goudineau, 1970, pl. III, $\mathrm{n}^{\circ} 40$ ). La variante $\mathrm{B}$ trouve son pendant plus tardivement à Pompéi (Volonté, 1984, p. 136, pl. 6, nº 5) dans un contexte augusto-tibérien, et en Grande-Bretagne, mais en tant que produit importé, probablement d'une région méditerranéenne (Peacock, 1977b, p. 157, fig. 3, $\mathrm{n}^{\circ} 4$, et p. 153-154). Des formes typologiques répertoriées sur les sites du limes, seule la forme 21B d'Oberaden

11. Ce type est le moins représenté à Genève avec $7.9 \%$ des céramiques à engobe interne. I auteur remarque que "les plats à bord épaissi, dont le $n^{\circ} 589$, plus rare, reproduisent une forme simplifice qui restera en usage jusqu’à la fin de l'époque romaine". 
(Albrecht, 1938, p. 37, pl. 26) est similaire à la variante C, mais avec un bord non épaissi ; or, cette dernière (bord aplati sur paroi convexe) est absente à la même époque à Dangstetten. La variante $\mathrm{C}$ est connue à Saint-Romain-enGal dans un contexte tibéro-claudien (Leblanc, 1994, p. 88 , fig. $86, n^{\circ} 4$ ) et elle est fréquente à Genève à l'époque augustéenne classique mais avec deux gorges sur la lèvre (Paunier, 1981, p. 259 et 367, n 586).

Il faut en déduire que les pratiques régionales, définissant la diversité typologique, prévalent à l'époque augustéenne précoce, cette diversité tendant à se réduire à l'époque augustéenne classique, si l'on admet comme résiduelle la présence du type 1 dans l'ensemble II, étant donné l'importance que prend le bord simple sur paroi concave (type 2) dans ce second ensemble.

\section{Type 2}

Cette forme à paroi concave et lèvre simple apparaît dans une seconde phase de production mise en évidence par l'étude des sigillées et des céramiques à parois fines (ensemble II). Elle prédomine sur les autres types et prend le relais du type 1 de façon soudaine à l'époque augustéenne classique (elle représente $30,8 \%$ des bords de l'ensemble II). Ce phénomène suggéré ici en contexte d'atelier se trouve conforté du fait de la présence relativement abondante de ce type à Dangstetten (Fingerlin, 1986 , fosse $176, \mathrm{n}^{\circ} 34$, p. 280 ; fosse $221, \mathrm{n}^{\circ} 25$, p. 301 ; fosse $393, n^{\circ} 11$, p. 365 ; fosse $585, n^{\circ} 60$, p. 463 ). Sur les sites de la région lyonnaise, ce type est bien présent aussi dans les contextes augustéens (Genin, 1993, fig. 16, p. 69, $\mathrm{n}^{\circ} 23$ à décor de stries concentriques sur le fond interne; Leblanc, 1994, fig. 82, p. $84, \mathrm{n}^{\circ} 2$ d'origine italique) et on le rencontre encore dans un niveau flavien à SaintRomain-en-Gal (Leblanc, 1994, fig. 94, p. 98, n 2 d'origine italique).

Du fait de son extrême simplicité, cette forme est bien répartie géographiquement et chronologiquement. Elle est attestée dans les camps rhénans augustéens : à Oberaden (Albrecht, 1938, p. 37, pl. 26, forme $21 \mathrm{~A}$ ), à Haltern (Loeschcke, 1909, p. 268-269, type 75 A, bord 6, pl. XIV) et à Dangstetten (Fingerlin, 1986, fosse 176, $\mathrm{n}^{\circ} 34$, p. 280 ; fosse $221, \mathrm{n}^{\circ} 25$, p. 301 ; fosse $393, \mathrm{n}^{\circ} 11$, p. 365 ; fosse $585, n^{\circ} 60$, p. 463 ). En outre, elle est répertoriée à Pompéi sous les règnes d'Auguste et de Tibère (Volonté, 1984, p. 136, pl. 86, $n^{\circ} 1$ et 2 à sillon sur la lèvre interne, $n^{\circ} 3$ et 4 , et $n^{\circ} 6$ et 7 de petit module), sur les sites hispaniques à l'époque augustéenne classique
(Vegas, 1964, type $15 \mathrm{C}$, p. 23), à Genève durant toute la première moitié du $\mathrm{I}^{\text {er }} \mathrm{s}$. (Paunier, 1981, p. 258 et 367 , $\mathrm{n}^{\circ} 578$ ) et elle est largement répandue en GrandeBretagne, caractérisée soit par une paroi plus ou moins convexe (Peacock, 1977b, p. 157, fig. 3, formes 1, 5, et 10, produites dans la région méditerranéenne et en GrandeBretagne), soit par une paroi rectiligne (Peacock, 1977b, p. 157, fig. 3, forme 8, peut-être produite en Centre Gaule et forme 12 probablement fabriquée à Colchester). Enfin, cette forme simple perdure sous le Haut-Empire avec une paroi rectiligne (Goudineau, 1970, p. 168, pl. II, $\mathrm{n}^{\circ} 29$, exemple provenant de Vindonissa daté de l'époque claudienne), voire rentrante (Goudineau, 1970, p. 168, pl. II, $\mathrm{n}^{\circ} 30$, exemple provenant d'Hofheim daté du règne de Vespasien).

Dans les régions du nord de la Gaule, une forme à lèvre simple est aussi bien représentée, mais le pied diffère nettement puisqu'il est rehaussé (Thuillier, 1993, p. 222, fig. $11, \mathrm{n}^{\circ} 1$ à 7 ).

\section{Type 3}

Ce type se définit par une paroi rectiligne oblique à tendance convexe, due à l'évasement du bord. Sur quelques fragments, le bord évasé est souligné par un bourrelet naissant. Ce bourrelet peut prendre des profils plus ou moins arrondis ou anguleux. À Lyon, la production de ce type se développe dans la seconde phase de production de l'atelier, mais elle reste secondaire (elle passe de $1 \%$ des bords de l'ensemble I à $9,6 \%$ dans l'ensemble II). Ce constat, confronté à l'aspect morphologique, incite à interpréter sa production comme une production de transition entre les types à paroi concave et les types à paroi convexe.

À l'inverse du type 2 , le type 3 , que représentent diverses variantes de lèvres, est moins répandu; il est notamment absent des régions hispaniques, de GrandeBretagne, de Genève, et on ne le trouve ni à Dangstetten, ni à Oberaden. Il est néanmoins attesté dans les niveaux précoces de Bolsena justement avec différents profils de lèvres (Goudineau, 1970, pl. VIII, $\mathrm{n}^{\circ} 14,15$ et 17 , couche 3 datée de 90 à 30 avant J.-C.; pl. VIII, $\mathrm{n}^{\circ} 7$, couche $2 \mathrm{C}$ datée de 40 à 10 avant J.-C.; pl. VIII, $n^{\circ} 3$, couche 2B datée de 20 à 10 avant J.-C.). Cette forme à bord évasé est aussi présente avec une lèvre à bourrelet plus ou moins bien formé à Haltern (Loeschcke, 1909, pl. XIV, n $75 \mathrm{~A}$, p. 275 , fig. 39 , bord 5 , forme simplement évasée et pl. $\mathrm{XIV}, \mathrm{n}^{\circ} 75 \mathrm{~B}$, forme intégrant une lèvre à bourrclet), où 
elle est associée à la forme de couvercle que nous avons classée sous le type 7 (couvercle à rebord).

\section{Type 4}

Sa morphologie est caractérisée par la lèvre, qui forme un rebord large à profil plus ou moins rectangulaire.

Cette forme nouvelle n'est pratiquement pas représentée dans l'ensemble I ( $0,2 \%$ des bords). Comme le type 3 , elle connaît une légère expansion au cours de la deuxième phase de production (avec seulement 1,4\% des bords représentés) qui ne peut être significative en raison de la trop faible représentation numérique. Il paraît donc prématuré de présumer des prémices de la production de ce type dans l'atelier à l'époque augustéenne classique. Le phénomène peut tout aussi bien être interprété comme un indice de résidualité du type dans les dépotoirs, d'autant plus que la forme, guère standardisée, apparaît de façon continue depuis la fin du $\mathrm{II}^{\mathrm{c}} \mathrm{s}$. avant J.-C. jusqu'au milieu du Irr s. après J.-C. (Volonté, 1984, p. 134). Il est d'ailleurs attesté à Saint-Romain-enGal dans un contexte tibéro-claudien (Leblanc, 1994, p. 88 , fig. $\left.86, \mathrm{n}^{\circ} 2\right)$.

Diverses formes approchantes à rebord, de facture plus fine, sont attestées dès la fin du $\mathrm{II}^{\mathrm{e}} \mathrm{s}$. avant $\mathrm{J}$.-C. dans les niveaux de Bolsena (Goudineau, 1970, p. 176, pl. VI). En outre, le type 4, bien représenté à l'époque augustéenne précoce à Dangstetten (Fingerlin, 1986, fosse 42, $\mathrm{n}^{\circ} 18$, p. 245 ; fosse $67, \mathrm{n}^{\circ} 11$, p. 256 ; fosse $155, \mathrm{n}^{\circ} 9$, p. 275 ; fosse $499, n^{\circ} 56$, p. 413 ; fosse $585, n^{\circ} 65,67,69$, 70, p. 463 et 464), à Saint-Romain-en-Gal (Leblanc, 1994, p. 82 , fig. $82, n^{\circ} 1$ avec une paroi très épaisse) et sur les sites de consommation lyonnais (Goudineau éd., 1989, site de la rue des Farges, p. 101 et site du Verbe-Incarné, p. 103) ${ }^{12}$, est très peu représenté dans les dépotoirs de la Muette. On le trouve aussi à Oberaden avec un rebord à profil triangulaire (Albrecht, 1938, pl. 26, n²2), à Pompéi (Volonté, 1984, p. 134, pl. 85, nº 7 et 8), et à Genève avec deux cannelures sur le rebord (Paunier, 1981 , p. 259 et $267, \mathrm{n}^{\circ} 588$ ).

Ce type de traitement de la lèvre, facilitant l'usage d'un couvercle, n'est pas répertorié en Grande-Bretagne. Bien qu'il soit impossible de restituer avec certitude l'assemblage du plat avec le couvercle en usage dans

12. Ce type apparaît notamment dans un contexte daté de 30 à 15 arant J.-C. sur le site lyonnais de la rue des Farges alors que la forme ancienne à bord en amande semble avoir déjà disparu.
l'Antiquité, il semble logique que les deux formes à rebord, qui s'adaptent aisément l'une à l'autre, soient associées (plat de type 4 et couvercle de type 7 ).

Étant donné que le type 4, minoritaire dans l'atelier, est bien représenté sur les sites de consommation, la confrontation avec le matériel d'habitat met en évidence une éventuelle fabrication du type 4 antérieurement au contexte de l'ensemble I dans l'atelier.

\section{Type 5}

C'est la forme de couvercle la mieux représentée de l'ensemble I $(35,7 \%$ des bords dans l'ensemble I pour $5,5 \%$ dans l'ensemble II). Elle possède un bord simple sur lequel une légère arête a tendance à se former pour assurer une meilleure adhésion au plat. Cette forme est évolutive et la naissance de l'arête aboutit à la formation du bord triangulaire du type 6 . Le type 5 comme le type 6 ont la même chronologie que les plats de type 1 et 2 .

Un type comparable est pour lors connu en GrandeBretagne (Peacock, 1977b, fig. 3, nº 9, attribué aux ateliers du centre de la Gaule) ${ }^{13}$.

\section{Type 6}

Il est affilié au type 5, dont la partie supérieure du bord est rendue horizontale, ce qui forme un profil de bord triangulaire. Bien que minoritaire dans les deux ensembles, il est convenablement représenté (19,8\% des bords dans l'ensemble I et $13,7 \%$ dans l'ensemble II). Ce type est attesté dans un contexte augustéen lyonnais (Genin, 1993, p. 82, fig. 29, n 325) et à Saint-Romain-enGal dans des contextes tardifs (Leblanc, 1994, p. 94, fig. $90, \mathrm{n}^{\circ} 2$ d'origine campanienne, p. 98 , fig. $94, \mathrm{n}^{\circ} 1$ originaire de Gaule du Centre et p. 103, fig. 97, $\mathrm{n}^{\circ} 7$ d'origine italique). On le connaît aussi à Pompéi à l'époque augustéenne classique (Volonté, 1984, p. 136, pl. 86, n 9 ) et à Colchester, où il pourrait avoir été produit (Peacock, 1977b, p. 157, fig. $3, \mathrm{n}^{\circ} 2$ et 11 ).

\section{Type 7}

Ce type est fabriqué sur le même principe que le type 6 mais avec un bord à profil rectangulaire, qui privilégie

13. La présentation de la forme comporte une erreur dorientation : il convient de l'inverser d'après nos fragments sur lesquels le pied annulaire est conservé. 
l'assemblage du plat au couvercle puisque le rebord s'adapte facilement sur un plat de type 4 notamment. Ce type de couvercle est produit dès l'époque augustéenne précoce (il représente $6,1 \%$ des bords de l'ensemble I), puis il prćdomine nettement à l'époque augustéenne classique (avec $22,6 \%$ des bords dans l'ensemble II). Le seul parallèle de ce type est un exemplaire découvert dans un contexte augustéen du Verbe-Incarné à Lyon (Genin, 1993 , p. 82 , fig. $29, n^{\circ} 326$ ).

\section{$*$ \\ * *}

La céramique à engobe interne rouge pompéien est une production annexe mais non anecdotique de l'atelier en activité à l'époque augustéenne. On a comptabilisé, dans les contextes étudiés, 1526 fragments de ce type de céramique, qui représentent environ $1 \%$ de la production de l'atelier (d'après le nombre de bords) contre $66 \%$ de céramique à parois fines et $33 \%$ de sigillée.

Les fosses-dépotoirs étudiées correspondent à un laps de temps si restreint qu'il est difficile de définir l'évolution typologique de cette catégorie céramique spécifique. Certains phénomènes nous semblent pourtant acquis : l'antériorité du type 4 par rapport aux autres qu'il fut ou non produit dans l'atelier ${ }^{1.4}$, la contemporanéité des types $1,2,5$ et 6 , et la continuité des types 1 et 2 sur les sites de consommation, notamment sous forme résiduelle, jusqu'à l'époque flavienne. De plus, l'absence, notamment dans l'ensemble I, de la forme à bord en amande, extrêmement répandue dans les contextes préaugustéens (à Genève, Paunier, 1981 , p. 258 et $367, n^{\circ} 581$ à 585 ; à Vintimille, Goudineau, 1970, pl. I, $\mathrm{n}^{\circ} 7$ et 8 , et pl. II, $\mathrm{n}^{\circ} 21$; à Bolsena, Goudineau, 1970, pl. VII, $\mathrm{n}^{\circ} 7$ et 8 , couche 3 ; à Pompéi, Volonté, 1984, p. 133, pl. 85, n 1 à $5)$, et relativement bien représentée sur les sites de la région lyonnaise dans un contexte daté de 40 à 20 avant J.-C. (Goudineau éd., 1989, p. 103, 115 et 118), tout comme dans les contextes helvétiques de cette même fourchette chronologique (Haldimann, 1991, p. 264), pourrait indiquer que la production, dans cet atelier, ne débute pas avant le règne d'Auguste.

14. En cffet, la faible representation de ce type dans notre lot incite a penser que ce type était produit antéricurement à l’époque augustéenne supplantant le type à lère en amande et cela, avant l’apparition des types à lère triangulaire ou à lère simple.
Par ailleurs, le répertoire des formes de ces productions lyonnaises s'inscrit, à la période augustéenne, dans un faciès typologique régional, qui semble concerner l'Italie du Nord, la haute vallée du Rhône et la Suisse, et le limes. En effet, nous avons noté des différences morphologiques importantes sur les productions de Gaule du Nord et de Gaule Belgique (Thuillier, 1993; De Laët, Thoen, 1969).

S. E.

\section{LES LAMPES}

L'échantillon de lampes est très limité. Il est constitué par les fragments de lampes exhumés lors des deux campagnes de fouille (1966 et 1975), qui sont issus des dépotoirs (Gobelet I, Gobelet II, D. I, D. II, T.N.E. et M.T.S.).

\section{TYPOLOGIE (fig. 83)}

En raison de la complexité morphologique de ce type de récipient, nous avons pris le parti de définir les types selon divers critères : le caractère discriminant du type (morphologie du bec) et des caractères annexes (profil du bec, profil du bandeau, aspect des volutes le cas échéant, raccord du bec au bandeau) qui déterminent les variantes. En outre, nous présentons les autres caractéristiques (morphologie de l'anse ou traitement du fond) susceptibles d'être des critères d'identification.

\section{CATALOGUE}

\section{TYPE I}

Ce type spécifique souvent assimilé par erreur au type Loeschcke IA est en fait le prototype de la forme Loeschcke IV. Il a été repéré notamment par A. Leibundgut (1977, $n^{\circ} 4$, p. 29-30) comme étant produit dès le début du règne d'Auguste, ce que nous sommes en mesure de confirmer pour la production de l'atelier de la Muette.

\section{Lampe complète en pâte orangée et vernis rougeâtre écaillé}

Diamètre : $7,5 \mathrm{~cm}$.

Sans trou d'évent. Sans anse. 


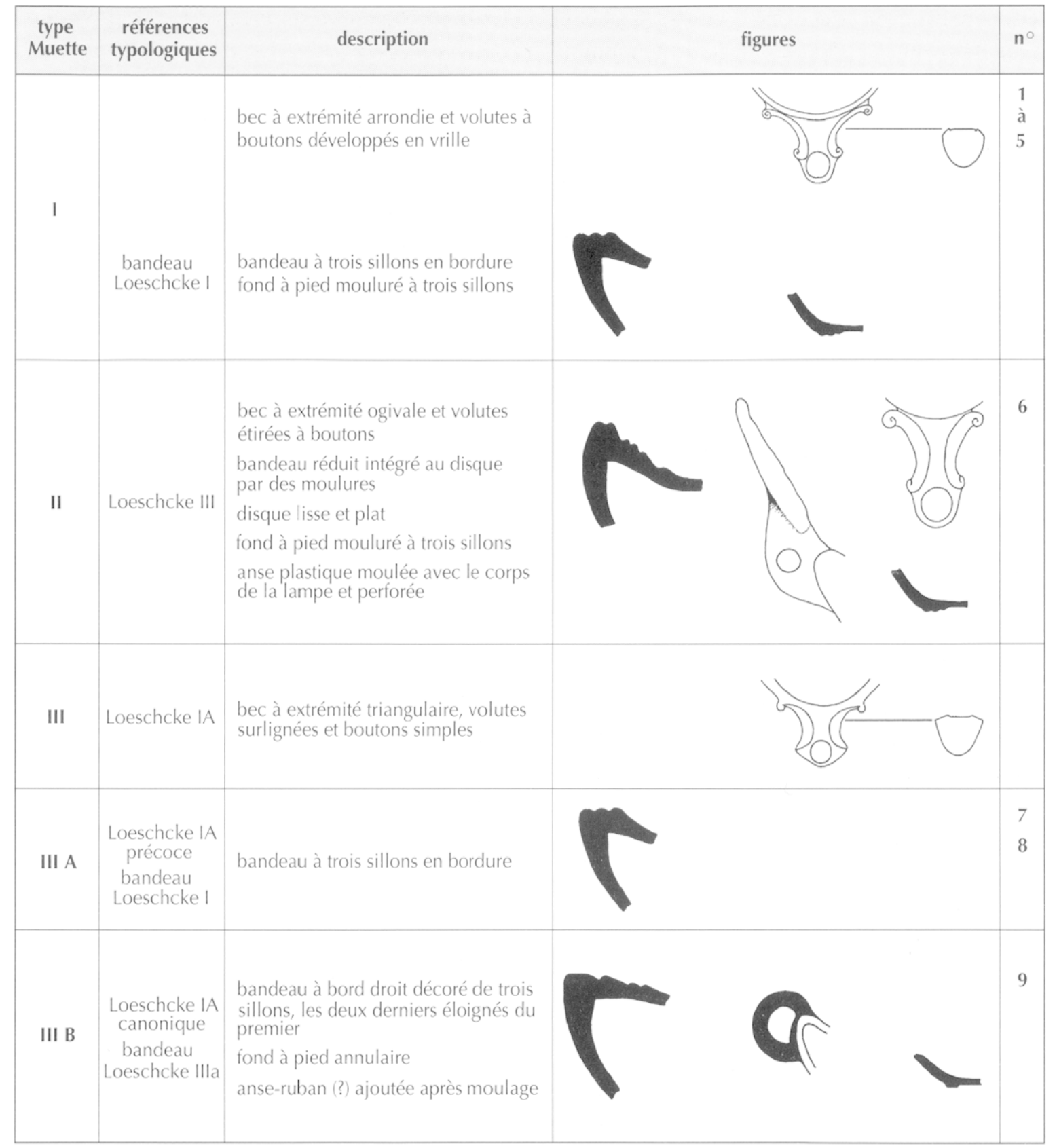

Fig. 83. Typologie des lampes.

Pustules de surmoulage au plâtre.

Médaillon : masque de Jupiter-Amon. Le trou de remplissage fait office de bouche (pl. 83 et 84 ).

Parallèles : $n^{\circ}$ 2, 3, 4 varaisemblablement fabriqués avec le même moule.

Ce motif iconographique (fabriqué semble-t-il avec un poinçon différent) a été trouvé à Lyon, dans les fouilles de Trion (Allmer, Dissard, $1888, n^{\circ} 1465$, p. 513 ) mais l'ourrage ne donne pas sa représentation exacte. Ce masque est par ailleurs connu à Narbonne (Solier, Sabrié, 1979, p. 50) avec un bandeau décoré d'oves, sur le même type. Il est figuré sur types Loeschcke IA et IB datés de l'époque tibérienne à Vindonissa (Leibundgut, 1977, pl. $26, n^{\circ} 31$, p. 137 et 217) et il est spécialement adapté à 
Trèves à une veilleuse de forme ovale à volutes et bec ogival (Goethert-Polascheck, 1985, type XIX, p. 151, motif 20 , p. 198) mais dans ces deux cas, il est fabriqué avec un poinçon différent. Enfin, il est attesté en Égypte, sur une lampe de type Loeschcke IV, entouré par un décor de points (Bailey, 1988, Q1907, p. 25-26 et 235) ${ }^{15}$.

\section{Médaillon en pâte orangée et vernis rougeâtre écaillé}

Diamètre : $7,5 \mathrm{~cm}$.

Sans trou d'évent. Sans anse.

Pustules de surmoulage au plâtre.

Médaillon : masque de Jupiter-Amon (pl. 83).

Parallèles : cf. $n^{\circ} 1,3,4$.

\section{Médaillon en pâte jaunâtre et vernis verdâtre, surcuit}

Diamètre : $7,5 \mathrm{~cm}$.

Pustules de surmoulage au plâtre.

Médaillon : masque de Jupiter-Amon (pl. 83).

Parallèles : cf. $n^{\circ} 1,2$ et 4.

Remarque : fragment déjà publié (Vertet, 1969, fig. 1 la, p. 116).

\section{Lampe complète en pâte beige sans revêtement et déformée}

Facture grossière (jusqu'à $0,7 \mathrm{~cm}$ d'épaisseur).

Diamètre : $7,5 \mathrm{~cm}$.

Pustules de surmoulage au plâtre.

Trou d'évent circulaire et large sur le disque.

Médaillon : masque de Jupiter-Amon (pl. 83).

Parallèles : cf. $\mathrm{n}^{\circ} 1,2$ et 3.

Remarque : lampe déjà publiéc (Elaigne, 1993, fig. 2, $\mathrm{n}^{\circ}$ 1, p. 241).

\section{Fragment de bandeau et volute associé à un fond en pâte pulvérulente beige à mica engobée en brun orangé}

Identification typologique aléatoire en raison de l'absence du bec.

Pustules de surmoulage au plâtre.

Marque en léger relief : $S$ ? (pl. 84).

Sans parallèle.

15. I auteur signale les trois excmplaires de l'atelier de la Muenc qui sont en vitrine au musée de la Civilisation gallo-romaine de lyon.

\section{TYPE II}

\section{Lampe quasi complète en pâte orangée et engobe} orangé

Diamètre : $6,2 \mathrm{~cm}$.

Anse-réflecteur non conservée. Trou d'évent en fente sur le bec.

Disque lisse (pl. 83 et 84 ).

Parallèles : deux exemplaires de ce type à moulures similaires et anse-plastique en forme de croissant de lune, datés de la fin de l'époque augustéenne, ont été découverts à Vindonissa (Loeschcke, 1919, fig. 4, p. 224) .

\section{TYPE III}

\section{Type IIIA}

On a rattaché deux exemplaires dont le bec manque au type IIIA par analogie avec des exemplaires du type Butte IA (type équivalent produit dans l'atelier de la Butte) et avec des exemplaires du site de la rue des Farges, qui associent les caractères typologiques secondaires suivants : bandeau à sillons en bordure, volutes surlignées à boutons simples, médaillon à décor en vogue à l'époque augustéenne (oves tournés vers l'extérieur ou rosette à pétales fins).

\section{Fragment de bandeau en pâte rosâtre et engobe brun}

Diamètre : $8 \mathrm{~cm}$.

Pustules de surmoulage au plâtre.

Décor du disque : non conservé mais cerné par des oves tournés vers l'extérieur (pl. 84).

Le décor d'oves sur le bandeau ou bien cernant le médaillon est caractéristique de l'époque augustéenne (on compte cinq exemplaires ainsi traités pour cette époque sur le site de la rue des Farges); ils sont tournés vers l'intérieur sur des types précoces (Loeschcke IA ou assimilés), puis ils sont remployés sur le bandeau du type Butte VIA mais toumés vers l'extérieur, à partir de l'époque tibérienne.

\section{Fragment de disque en pâte rosâtre à caractère pulvérulent et engobe brun orangé}

Diamc̀tre : $7 \mathrm{~cm}$.

Pustules de surmoulage.

Décor : rosette à pétales fins, doublés et non fermés autour du trou de remplissage (pl. 84). 
Parallèles : le décor végétal stylisé est fréquemment employé à l'époque augustéenne sur les types à volutes précoces (Loeschcke, 1919, pl. III, n 581 ; GoethertPolascheck, 1985, pl. 16, n 40, motif 28 ; Bailey, 1980 , Q760-788-1022, p. 86). Ce décor de rosette à pétales multiples existe sur un exemplaire du contexte augustéen du site de la rue des Farges et il y est aussi représenté sur six exemplaires de ce même site dans le courant du $\mathrm{I}^{\mathrm{cr}}$ s., parfois fermé par des cercles concentriques. Enfin, il est connu à Trèves sur un type à bec triangulaire et canal (Goethert-Polascheck, 1985, pl. 16, $\mathrm{n}^{3} 9$ ).

\section{Type III B}

On a attribué à ce type un exemplaire dont le bec manque mais qui présente un bandeau caractéristique généralement associé à un bec triangulaire, du moins à l'époque augustéenne.

\section{Lampe fragmentaire en pâte beige pulvérulente avec des inclusions de mica et des points de chaux, engobe orangé}

Diamètre : $6,8 \mathrm{~cm}$.

Trou d'évent en fente sur le bec.

Anse non conservée (anse-ruban ¿) appliquéc après moulage (pl. 84).

\section{MÉDAILLONS HORS TYPOLOGIE}

\section{Fragment de médaillon en pâte beige, engobe brun orangé}

Décor : zone à bâtonnets rayonnants limitée par des cercles concentriques autour du trou de remplissage (pl. 84).

Parallèles : cf. Butte 35 et 51 . Ce genre de décor est fréquent sur des types à bec triangulaire précoces de cette époque. Il est attesté sur trois exemplaires à l'époque augustéenne sur le site de la rue des Farges et il y est représenté sur sept exemplaires à partir de l'époque tibérienne.

\section{Fragment de médaillon en pâte beige à caractère pulvérulent, engobe orangé}

Décor : Mercure au chapeau ailé (pl. 83 et 84).
Parallèles : ce décor, commun, existe sur un fragment provenant du dépotoir de l'atelier de la Butte (cf. Butte, $\left.n^{\circ} 49\right)$ et sur un type Butte II (Loeschcke IB) daté des années 50/60 du site de la rue des Farges. On le retrouve à Alba sur un type Butte VI (Loeschckc VIII) de façon exceptionnelle (Ayala, 1990, p. 199, fig. 16, $\mathrm{n}^{\circ} 71$ ), à Vindonissa durant tout le $I^{\text {cr }}$ s. sur type Butte II (Loeschcke IB, cf. Loeschcke, 1919, pl. IV, $\mathrm{n}^{\circ} 4$; Leibundgut, 1977, pl. 26, motif 35, p. 138), à Trèves sur type Muette III (Loeschcke IA) et Butte II (Loeschcke IB, cf. Goethert-Polascheck, 1985, pl. 16 et 23, motif 28 , p. $200, \mathrm{n}^{\circ} 40$ et 173 ), et il est signalé en Italie sur type Butte II (Bailey, 1980, pl. 37, Q1059, p. 12) et à Carthage sur type Butte III (Loeschcke IC) et Butte V (Loeschcke IV, cf. Deneauve, 1969, pl. XXXV, n 279 et pl. XLV, II 406 ).

Ces quelques exemplaires indiquent que l'atelier produit aussi en série des lampes à huile à une époque précoce, même si cette production paraît, dans l'état actuel de nos connaissances, peu importante. Malgré la paurreté de l'échantillon qui ne permet pas de connaître la répartition typologique au sein des dépotoirs ou même de l'atelier sur les deux périodes de production, on remarque que les types Muette I et probablement des types Muette III étaient principalement produits.

D'après les exemplaires 1 et 2 et un fragment de cuve à vernis grésé de qualité, les lampes ont parfois bénéficié de la technique employée pour la production des sigillées : une pâte calcaire à revêtement argileux grésé. Certains potiers producteurs de sigillée auraient pu fabriquer des lampes de façon sporadique en utilisant un seul ou quelques moules spécifiques.

Cette production bien que précoce a été effectuée la plupart du temps au moyen du surmoulage au plâtre. Le motif au masque de Jupiter-Amon, thème très répandu, pourrait avoir été introduit à Lyon et surmoulé pendant quelques années dans l'atelier. Mais si le motif lui-même a été diffusé, nous n'avons nulle part la preuve que la production propre à la Muette, employant ce poinçon-décor, a été exportée, puisque tous les exemples connus à l'extérieur de Lyon (sauf peut-être celui de Narbonne) ont été fabriqués arec des poinçons différents. 
Dans l'état actuel des connaissances, nous ne pouvons que supposer qu'il s'agit d'une production d'appoint effectuée par des potiers non spécialisés.

Notons qu'une lampe de forme intacte a été découverte dans une fosse à argile postérieurc au pctit four augustéen. Celle-ci portant des traces de combustion sur le bec, fut utilisée dans l'atelier, mais rien ne prouve qu'elle fut fabriquée sur place.

S. E.

\section{LES AUTRES PRODUCTIONS}

À côté des céramiques qui représentent la majorité du mobilier collecté, et dont la fabrication sur place est assurée, figurent d'autres céramiques dont l'origine lyonnaise ne fait aucun doute, mais il n'est pas certain qu'elles aient été fabriquées sur le site même de la Muette.

Deux catégories sont concernées : les céramiques communes et les amphores. Il nous a semblé utile de présenter ces catégories qui apportent des éléments précieux pour la connaissance des productions lyonnaises.

\section{LA CÉRAMIQUE COMMUNE CLAIRE}

La céramique commune claire a été étudiée dans les ensembles : Gobelet I, Gobelet II, T.N.E., M.T.S., M.U. 66, S3/S4, M.U. 75 5b, M.U. $756 \mathrm{~d}+$.

L'ensemble de cette céramique compte 1604 fragments de vases et 197 formes, dont 924 fragments et 126 formes en céramique commune claire. L'importance de la commune claire par rapport à la commune sombre est inhabituelle pour cette époque sur les sites de consommation de type habitat et pourrait fournir un élément pour étayer l'hypothèse d'une production de commune claire à l'époquc augustćcnne dans l'atelier de la Muette alors que les céramiques étudiées ne présentent aucun signe de surcuisson ou autre indice de mise au rebut. Cette supposition est d'ailleurs confortée par le fait que cet atelier a fabriqué de la céramique commune claire à une période plus récente : deux fours de potiers dont l'un contenait, semble-t-il, sa dernière fournée (Lasfargues, 1976) datée de la fin du $\mathrm{I}^{\text {cr }}$ s. après J.-C. et du début du II $^{\mathrm{e}} \mathrm{s}$. Aucun indice de fabrication sur place ne permet d'inclure la céramique commune sombre à la production de la Muette.

Ces céramiques communes provenant de dépotoirs comblés en grande majorité de sigillées sont effectivement des céramiques utilisées dans l'atelier pour la consommation usuelle : cruches, mortiers. Les formes dont la fonction est uniquement liée à l'atelier (les supports de vases) ont été exclues de la typologie de la production car il ne s'agit pas, dans ce cas-là, de produits pour la vente contrairement aux autres formes (cruches, coupes et mortiers) qui ont été repérées sur des sites de consommation lyonnais contemporains.

\section{PréSentation (tabl. IX)}

Les deux grands ensembles (I et II) proposés lors de l'étude de la sigillée (cf. supra) contiennent chacun approximativement la même quantité de céramique : 486 fragments et 66 formes pour l'ensemble I et 438 frag-

\begin{tabular}{|l|c|c|c|c|c|c|c|c|c|c|c|c|c|}
\hline C.Claire & T1 & T2 & T3 & T4 & T5 & T6 & T7 & T8 & T9 & T.U. & n.a. & formes & fragments \\
\hline MU 66 GOB I & & & & & 1 & 1 & 25 & 16 & & & 5 & 48 & 401 \\
\hline MU 66 GOB I & & & & & & & 3 & 3 & & & 1 & 7 & 18 \\
\hline MU 66 TNE & 5 & 4 & 2 & 2 & & 6 & 1 & 15 & 2 & 1 & 5 & 43 & 365 \\
\hline MU 66 MTS & 1 & & & & & 3 & 4 & & & & 2 & 10 & 44 \\
\hline MU 66 S3/S4 & & & & & & 2 & 3 & 1 & & & 1 & 7 & 29 \\
\hline MU 75 5b & & & & & & 1 & 4 & & & 1 & 4 & 10 & 48 \\
\hline MU 75 6d+ & & & & & & & & & & 1 & & 1 & 19 \\
\hline total & 6 & 4 & 2 & 2 & 1 & 13 & 40 & 35 & 2 & 3 & 18 & 126 & 924 \\
\hline
\end{tabular}

\begin{tabular}{|l|c|c|c|c|c|c|c|c|c|c|c|c|c|}
\hline C.Claire & T1 & T2 & T3 & T4 & T5 & T6 & T7 & T8 & T9 & T.U. & n.a. & formes & fragments \\
\hline Ensemble I & & & & & 1 & 2 & 32 & 19 & & 2 & 10 & 66 & 486 \\
\hline Ensemble II & 6 & 4 & 2 & 2 & & 11 & 8 & 16 & 2 & 1 & 8 & 60 & 438 \\
\hline
\end{tabular}

Tabl. IX. Céramique commune claire de l'atelier de la Muette, données quantitatives et répartition typologique. 
ments et 60 formes pour l'ensemble II. Ils se distinguent l'un de l'autre par les formes de céramiques qui les composent; les deux ensembles comprennent une majorité de couvercles et de coupes, mais les cruches sont présentes uniquement dans le mobilier de l'ensemble II.

Les contextes M.U. 66 Gobelet I (ensemble I) et M.U. 66 T.N.E. (ensemble II) sont de loin les plus importants en nombre; T.N.E. est également le plus diversifié car il contient tous les types de céramiques définis dans cet atelier excepté le " pot à miel " qui est déjà minoritaire dans les autres contextes.

\section{TYPOLOGIE}

\section{Type 1}

Cruche à une anse, col étroit et lève striée (pl. 86, 1, 2 et 14). Six exemplaires. C'est la cruche la plus courante de l'époque augustéenne jusqu'au premier quart du $\mathrm{I}^{\mathrm{or}} \mathrm{s}$. après J.-C. non seulement dans la région lyonnaise (Desbat et alii, 1979, pl. III, ${ }^{\circ} 1-2$, pl. V, n ${ }^{\circ} 1-2$; Desbat $e t$ alii, 1989, fig. $66, \mathrm{n}^{\circ} 13$, fig. $70, \mathrm{n}^{\circ} 63$, fig. $73, \mathrm{n}^{\circ} 24$, fig. 75 , $n^{\circ} 8$; Genin, 1993, fig. 17, $n^{\circ} 24-25$, fig. $25, n^{\circ} 266$ ) et viennoise, mais de façon générale sur les sites de cette période : Besançon (Guilhot et alii, 1992, phase 4, p. 281, $\mathrm{n}^{\circ} 238$ ) et les camps rhénans : Haltern (Loeschcke, 1909, type 45), Oberaden (Albrecht, 1942, pl. 29, 43), Vindonissa (forme 429), Neuss (Filtzinger, 1972, forme 13), Camulodunum (forme 140 AC).

\section{Type 2}

Cruche à une anse, col étroit et lèrre arrondie (pl. 86, 3). Quatre exemplaires. Cette forme se retrouve dans les mêmes contextes régionaux (Desbat et alii, 1979, pl. III, $\left.n^{\circ} 3\right)$ mais en moindre quantité que le type précédent.

\section{Type 3}

Cruche à deux anses, col large et lèvre arrondie (pl. 86, 6). Deux exemplaires. C'est la cruche à deux anses la plus utilisée de la période augustéenne jusqu'au milieu du $\mathrm{I}^{\mathrm{er}} \mathrm{s}$. après J.-C. Elle se trouve aussi bien à Lyon (rue des Farges, Desbat et alii, 1979, pl. III, nº 6), à Vienne (réserve de céramiques d'époque de Claude, codard, 1992a, pl. VIII, 109) que dans les camps du limes: Haltern (Loeschcke, 1909, type 50), Hofheim 57, Vindonissa 500, (Tomasevic, 1970), Neuss (Filtzinger, 1972, type 21),
Oberaden (Loeschcke, 1942, pl. 30, 49), Rödgen (Schönberger, Simon, 1976, pl. 21, 416) ou toute autre installation augustéenne comme Camulodunum 167.

\section{Type 4}

Cruche à deux anses, col large et lève striée (pl. 86, 4). Deux exemplaires. Présente dès le règne d'Auguste à Lyon : contexte B3 de la rue des Farges (Desbat et alii, 1979, pl. V, $\mathrm{n}^{\circ} 4$ ) et sur le site du Verbe-Incarné (Genin, 1993, fig. 26, n²73), et à Vienne, notamment à SaintRomain-en-Gal, dans le dépotoir D3 de l'atelier de potier (Leblanc, 1992, pl. 75), ainsi qu'à Besançon (Guilhot et alii, 1992, phase 4, p. 281, n²39). Comme la cruche précédente, elle est utilisée dans les camps du limes: Haltern 53, Oberaden (Albrecht, 1942, pl. 31, 51), Vindonissa 486, Neuss 19, Bâle (Fellmann, 1955, pl. 7, 11) et à Camulodunum $163 \mathrm{AB}$.

\section{Type 5}

Pot à deux anses à lèrre plate (pl. 85, l et pl. 86, 5). Un exemplaire. Cette forme est à rapprocher du " pot à miel » augustéen de type Haltern 62 utilisé dans les camps comme les cruches à deux anses : Haltern (Loeschcke, 1909), Hofheim (type 66, Ritterling, 1913), Vindonissa (type 107/109), à Neuss (type 8, Filuinger, 1972). C'est également une forme courante à Camulodunum (type 175), Lyon (Desbat et alii, 1979, pl. II, 6), à Vienne (Godard, 1992a, pl. VII, n 110 et 111), à Besançon (Guilhot et alii, 1992, p.223, n ${ }^{\circ} 88$, p. $235 \mathrm{n}^{\circ} 183$ ) et en Bourgogne (Joly, 1994, p. 311, n 47 ).

\section{Type 6}

Mortier à lèvre en bandeau, généralement sans sable sur la paroi interne (pl. 85, 3 et 10 ; pl. 86, 7-9 et 16-18). Treize exemplaires. Ce récipient est abondant sur les sites d'ateliers de potiers où il devait servir à des préparations de vernis ou de barbotine. Ce type à lève en bandeau daté de la fin du $\mathrm{I}^{\mathrm{er}}$ s. avant J.-C. (Desbat et alii, 1979, pl. II, $n^{\circ} 6$ et pl. III, $n^{\circ} 11$; Genin, 1993, fig. 26, $n^{\circ} 281-283$ et fig. $27, n^{\circ} 284-288$ ) est remplacé au cours du siècle suivant par celui à lèrre pendante après une période commune comme le montre la réserve viennoise (Godard, 1992a, pl. XIII, n 149). Fréquent sur les sites d'époque augustéenne (Besançon phase 4 : Guilhot et alii, 1992, p. 281, $\left.\mathrm{n}^{\circ} 244\right)$, il fait partie de la cuisine des camps rhénans : 
Haltern 59, Hofheim 79, Camulodunum 191, Vindonissa $561 / 563$, et Neuss où une fabrique est attestée (Filtzinger, 1972). Sur l'atelier de Sallèles-d'Aude il est daté des années 20/60 après J.-C. (Laubenheimer, 1990, type B7).

\section{Type 7}

Couvercles avec bouton de préhension (pl. 85, 5-6; pl. 86, 10). Quarante exemplaires. La moitié des exemplaires ont le fond percé (pl. 86, 5).

\section{Type 8}

Couvercles/coupes sans bouton de préhension (pl. 85, 7-8; pl. 86, 11 et 13). Trente-cinq exemplaires. Comme pour le type précédent, la moitié des formes présentent un fond troué (pl. 85, 7-8; pl. 86, 13).

\section{Type 9}

Couvercle d'amphore (pl. 86, 12). Deux exemplaires. (Lattara, 6, 1993, CL-REC-16).

Ces couvercles avec ou sans bouton de préhension, qui peuvent souvent être utilisés comme des coupes, sont très abondants dans les contextes Gobelet I et T.N.E. Leur proportion inhabituelle par rapport à l'ensemble de la commune claire $(60,8 \%)$ laisse penser qu'une partie au moins devait avoir une fonction spécifique dans l'atelier (supports de vases pendant le séchage?). Si ces types ont été conservés dans la typologie de la production, alors qu'à priori les ustensiles de fabrication en étaient exclus, c'est que certains sont certainement de véritables couvercles et qu'ils ont pu être commercialisés comme tels.

\section{Types uniques}

- pot à col légèrement déversé (pl. 85, 2) très semblable au « pot à saumure » du dépotoir D5 de l'atelier de SaintRomain-en-Gal (Leblanc, 1992, pl. 76),

- jatte (pl. 85, 4),

- grand pot à deux anses avec une lèvre présentant une rainure à l'intérieur (pl. 85, 9),

- un pot globulaire à fond plat dont le col est souligné de deux stries horizontales (pl. 85, 11).

L'étude typologique confirme la datation proposée à l'issue de l'étude de la sigillée (cf. supra); il n'est cependant pas possible de distinguer une différence chronolo- gique entre les deux périodes de production, même si le contenu des deux ensembles n'est pas semblable. Quel que soit le dépotoir de provenance, ces céramiques sont très fragmentaires et les formes n'ont pas toujours pu être identifiées. Dans la plupart des cas, elles appartiennent au répertoire connu des formes de l'époque augustéenne des sites d'habitat lyonnais. Cependant, quelques formes (pl. 85, 4, 9, 11) sont uniques et inconnues sur les sites de consommation, ce qui est souvent le cas dans un contexte d'atelier de potiers.

L'atelier lyonnais de la Muette a produit des céramiques communes claires dont les formes sont très connues non seulement sur les sites d'habitat lyonnais mais aussi sur un grand nombre d'agglomérations de l'Empire romain situées principalement sur les axes Rhône/Saône et Rhône/Rhin, sur le limes germanique et à Colchester en Grande-Bretagne.

La diffusion de la céramique commune claire de cet atelier est sans doute restée très locale, d'autres ateliers de même nature offrant les mêmes produits étaient installés dans des agglomérations voisines comme Vienne qui était fournie en céramique commune claire par des ateliers situés dans la ville même.

C. L.

\section{LES AMPHORES}

Parmi le matériel récolté dans les fouilles de 1966 et 1975 , on compte des amphores trouvées en association avec les autres catégories de matériel ou utilisées en réemploi. Plusieurs constituaient ainsi un drain le long d'un mur (Lasfargues, Vertet, 1976). À côté d'amphores importées, notamment de la péninsule Ibérique (Dressel 20, Dressel 7/11, Pascual, Dressel 12, Haltern 70...), on trouve une série d'amphores dont l'origine lyonnaise a été démontrée par les analyses (Dangréaux et alii, 1992; Schmitt, 1993).

Comme on l'a déjà signalé, il n'est pas certain que ces amphores aient été produites sur le site même de la Muette. Elles peuvent provenir d'un site de production voisin comme celui contigu de la Manutention militaire où d'autres découvertes semblables ont eu lieu.

Les pâtes, de couleur beige et d'aspect grossier, sont semblables à celles d'autres céramiques calcaires dites " à pâte grossière " trouvées dans l'atelier, mortiers et ustensiles utilisés par les potiers. Ces derniers objets sont de trois sortes : plaquettes d'argile, récipients en forme de 
coupe à bord arrondi, plateaux cylindriques dont la surface est légèrement creusée de rainures concentriques.

Les amphores se classent en trois catégories qui sont décrites ci-dessous.

\section{LES DRESSEL 2/4 (pl. 87)}

Les amphores de ce type ne sont pas très nombreuses sur le site et une dizaine d'exemplaires seulement ont été recensés, dont un seul exemplaire complet. Presque toutes appartiennent au type $2 \mathrm{~B}$.

Ces amphores sont caractérisées par une lèvre arrondie, un col haut, rétréci à la base, une épaule à la carène prononcée, des anses pseudo-bifides, un pied plein marqué par un ressaut caractéristique, que l'on retrouve sur les amphores du Falerne, dont ces amphores s'inspirent à l'évidence.

Un seul fond ( $p l .87,7$ ) de forme arrondie, terminé par une petite protubérance cylindrique creuse, pourrait appartenir à une amphore de type $2 \mathrm{~A}$, dont la forme s'inspire du type de Cos. Ce type, moins courant que le $2 \mathrm{~B}$, est connu par un exemplaire complet du dépôt de la rue de la Favorite (Becker et alii, 1986).

\section{LES AMPHORES À SAUMURE (pl. 88)}

Le deuxième type d'amphore est représenté par une dizaine d'exemplaires, couramment désignés sous le nom de Dressel 9 similis, correspondant aux types 3A et 3B des amphores lyonnaises (Dangréaux et alii, 1992). La majorité des exemplaires appartient au type 3A, qui est caractérisé par une lèvre légèrement évasée, débordante, soulignée par un ressaut plus ou moins marqué (1 à 6). Les anses sont toujours creusées d'un sillon.

Une seule lèvre, hors contexte, appartient au type 3B, qui apparaît à une date plus récente (8).

\section{LES AMPHORES À FOND PLAT (fig. 84)}

Ce type d'amphore apparenté au type Dressel 28 est connu par cinq amphores prises dans le mur d'un bassin qui constituait sans doute un vivier. Les deux exemplaires conservés présentent deux caractéristiques : fond plat ombiliqué et à pied annulaire. La panse est ovoïde, le col court surmonté par une lèvre en poulie. Les anses plates comportent deux sillons.

\section{LES ESTAMPILLES}

Parmi le matériel récolté figurent également deux estampilles.

1. FAVSTI, dans un cartouche rectangulaire (pl. 88, 9). Cette marque inédite figure sur un fragment de col court qui pourrait appartenir à une forme à fond plat.

2. LICINVS dans un cartouche rectangulaire (pl. 88, 10). Marque sur col cylindrique appartenant à une Dressel 2/4. La marque est inédite sous cette forme, mais on connaît une estampille circulaire avec la même marque sur une amphore Dressel 9 similis de Roanne.

A. D., B. D.

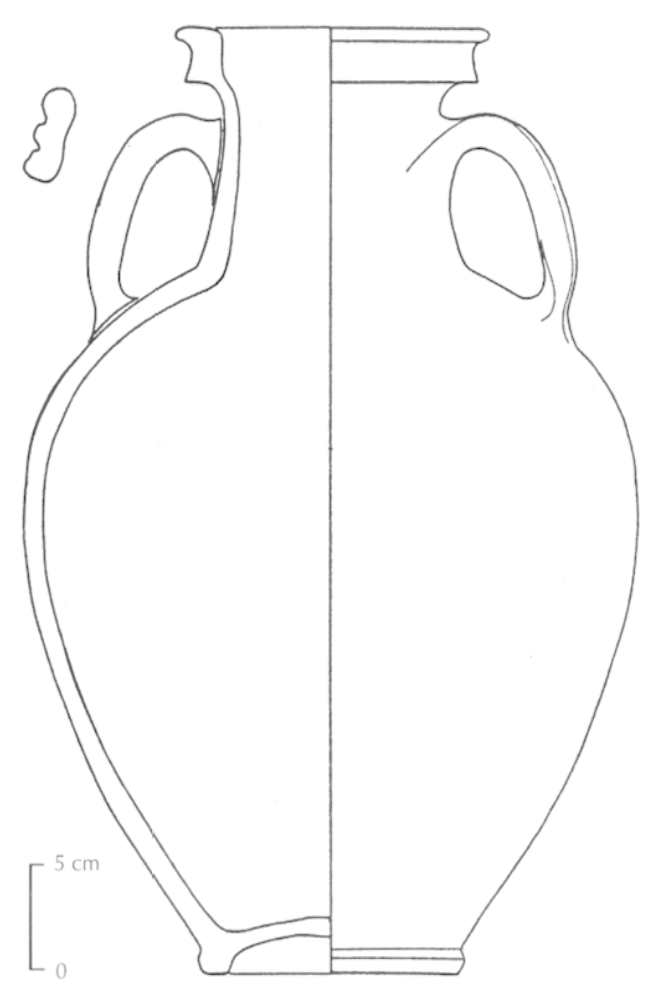

Fig. 84. Amphore à fond plat. 


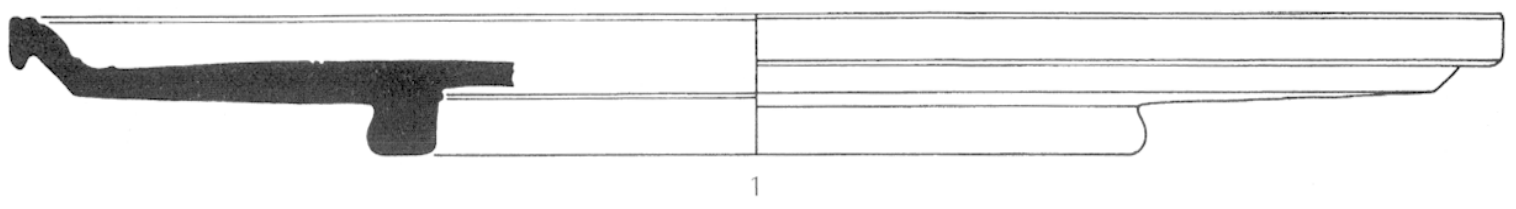

\section{PI. 9. ENSEMBLE I}

\section{1-5 : plats du service IB (53 ex.)}

Ces plats présentent des profils comparables aux formes les plus répandues de cette série en Italie comme sur le limes:

- lèvres droites, rainurées à leur extrémité supérieure et division bipartite interne marquée par un renflement très épais (1-4),

- lèvres légèrement arrondies soulignées à I'intérieur par une gorge et division bipartite interne (5).

1 : Goudineau, 1968, type 15; Ettlinger, 1983, pl. 3, n० 15-26; Schindler, Scheffenegger, 1977, pl. $22, n^{\circ} 1-2$.

2: Ettlinger, 1983, pl. 3, 7-8; Fingerlin, 1986, 444-4.

3: von Schnurbein, 1982, pl. 1, $n^{\circ} 2$; Schindler, Scheffenegger, 1977, pl. 22, $n^{\circ} 3$ (mais attache bord/fond arrondie); Genin, 1993, fig. 16, n 1 .

\section{ASSIETTES DU SERVICE IB (913 ex.)}

Groupe $1: 844$ ex. $(92,5 \%)$

Groupe $2: 69$ ex. $(7,5 \%)$

6-18 : assiettes du service IB, groupe 1 (844 ex.) Le groupe 1 montre plusieurs séries comprenant aussi bien des formes précoces du service IB que des variantes encore peu standardisées se rapprochant de profils plus couramment attestés sous Auguste.

11-13 : Ettlinger, 1983, pl. 11, n० 3-4.

15 : Ettlinger, 1983, pl. 10, n' 12-13; Fingerlin, 1986, 374-9, 401-19.

16-18 : Ettlinger, 1983, pl. 12, n 18; Fingerlin, 1986, 552-22.
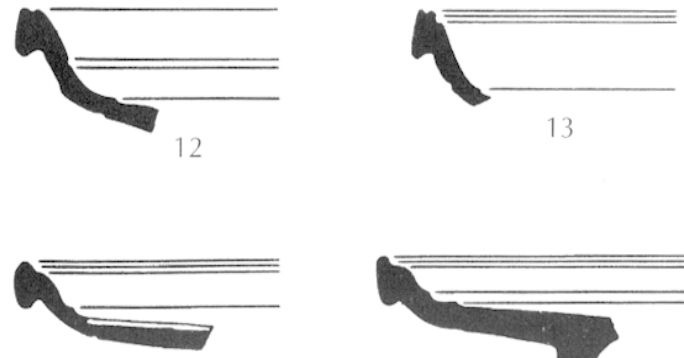

16
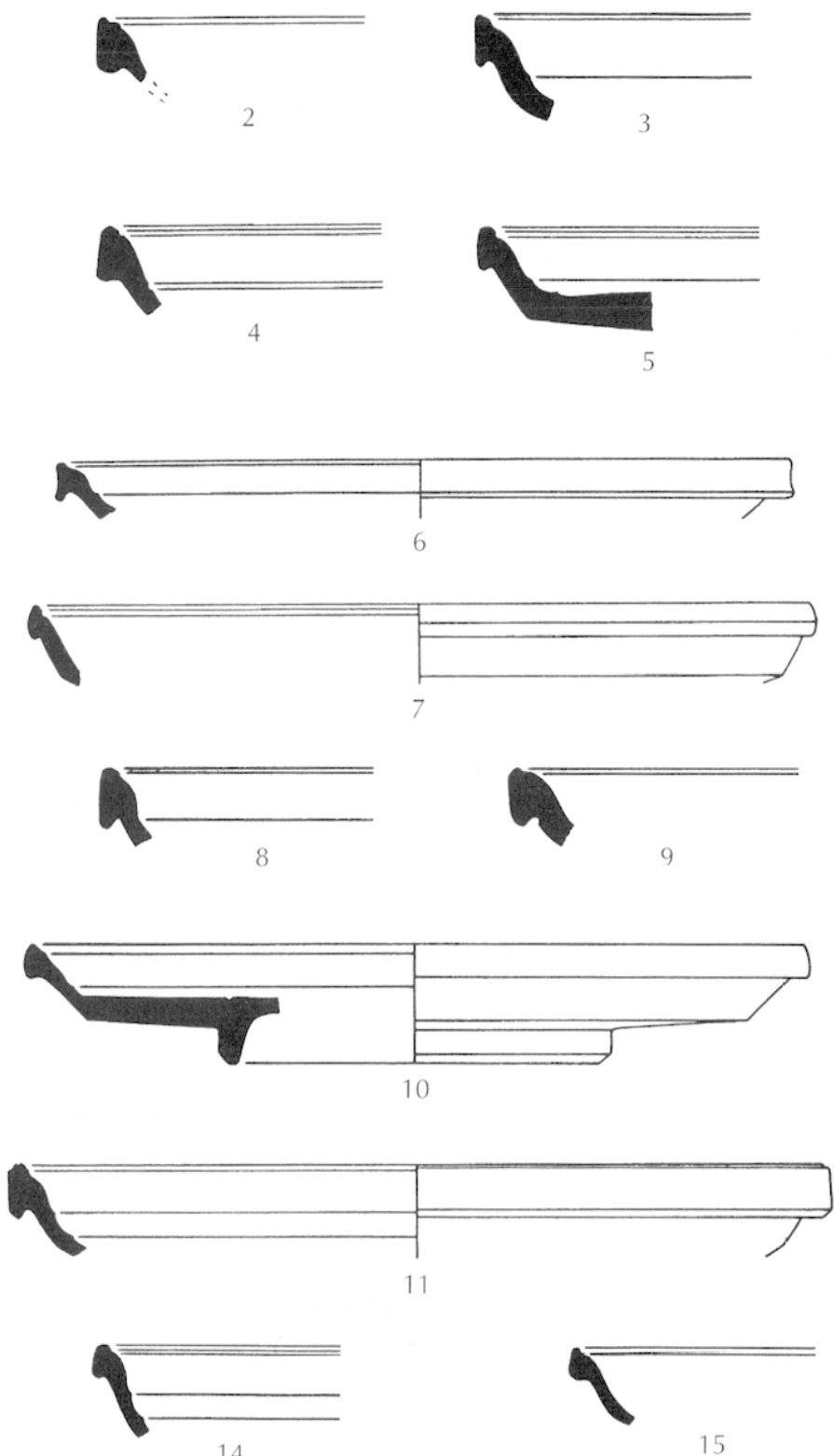

14

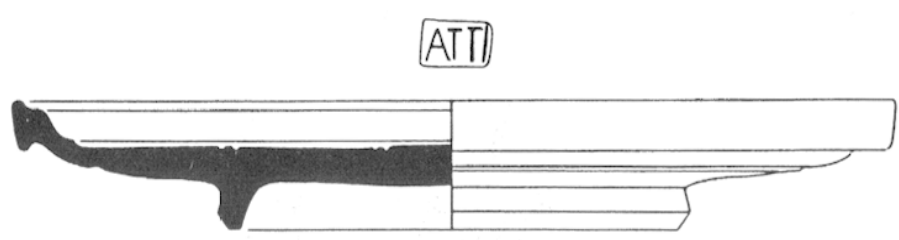

18

P1. 9. Ensemble I, sigillée, service IB : plats (1-5), assieltes du groupe 1 (6-18) (échelle : 1/2). 


\section{PI. 10. ENSEMBLE I}

1-4 : assiettes du service IB, groupe 2 (69 ex.) Le groupe 2 constitue à l'inverse une série très homogène. Les lèvres sont droites, relativement hautes, collées à la paroi et dégagées à leur extrémité supérieure par un bourrelet bien marqué ; la paroi, parfaitement oblique, se raccorde au fond par un angle vif. Ce type d'assiettes inconnu à Bolsena semble précisément correspondre par les profils des lèvres aux bols IB appelés "bols type Lyon " dans les publications allemandes. II est d'ailleurs intéressant de noter l'absence quasi totale de ces formes $d^{\prime}$ assiettes sur les sites de consommation où les bols "type Lyon "sont, quant à eux, bien représentés dans la plupart des cas.

Ettlinger, 1983, pl. 12, $n^{\circ} 8$.

\section{BOLS DU SERVICE IB (4 502 ex.)}

Les bols du service IB se rattachent à cinq groupes distincts.

Les groupes 1 et 3 comprennent des variantes du service IB encore faiblement standardisées pour lesquelles on peut cependant établir des parallèles dans le mobilier du limes. A l'inverse, le groupe 2 qui se singularise par une relative homogénéité typologique ne semble pas attesté sur les principaux sites de référence. Au groupe 4 correspondent les "bols type Lyon » et au groupe 5 des profils satellites des profils type Lyon.

Girnupe $1: 341$ ex. $(7,5 \%)$

Groupe 2 : 85 ex. (2\%)

Groupe $3: 210$ ex. (5\%)

Groupe $4: 2274$ ex. $(50,5 \%)$

Groupe 5 : 1592 ex. $(35 \%)$.

\section{5-15 : bols du service IB groupe 1 (341 ex.)}

Lèvres courtes de section arrondie à face externe légèrement oblique, dégagées à l'intérieur soit par une gorge, soit par un simple sillon; l'attache bord/fond est dans tous les cas de profil arrondi ; paroi interne non divisée ou à division bipartite variable. Presque tous les exemplaires de ce groupe sont des bols de petit calibre dont les ciamètres avoisinent 80-100 mm.

Goudineau, 1968, type 16; Ettlinger, 1983, pl. 31, $n^{\circ} 5-6, p l .30, n^{\circ} 22-23$ (en général) et pl. 29, 17 $\left(n^{\circ} 3\right)$.
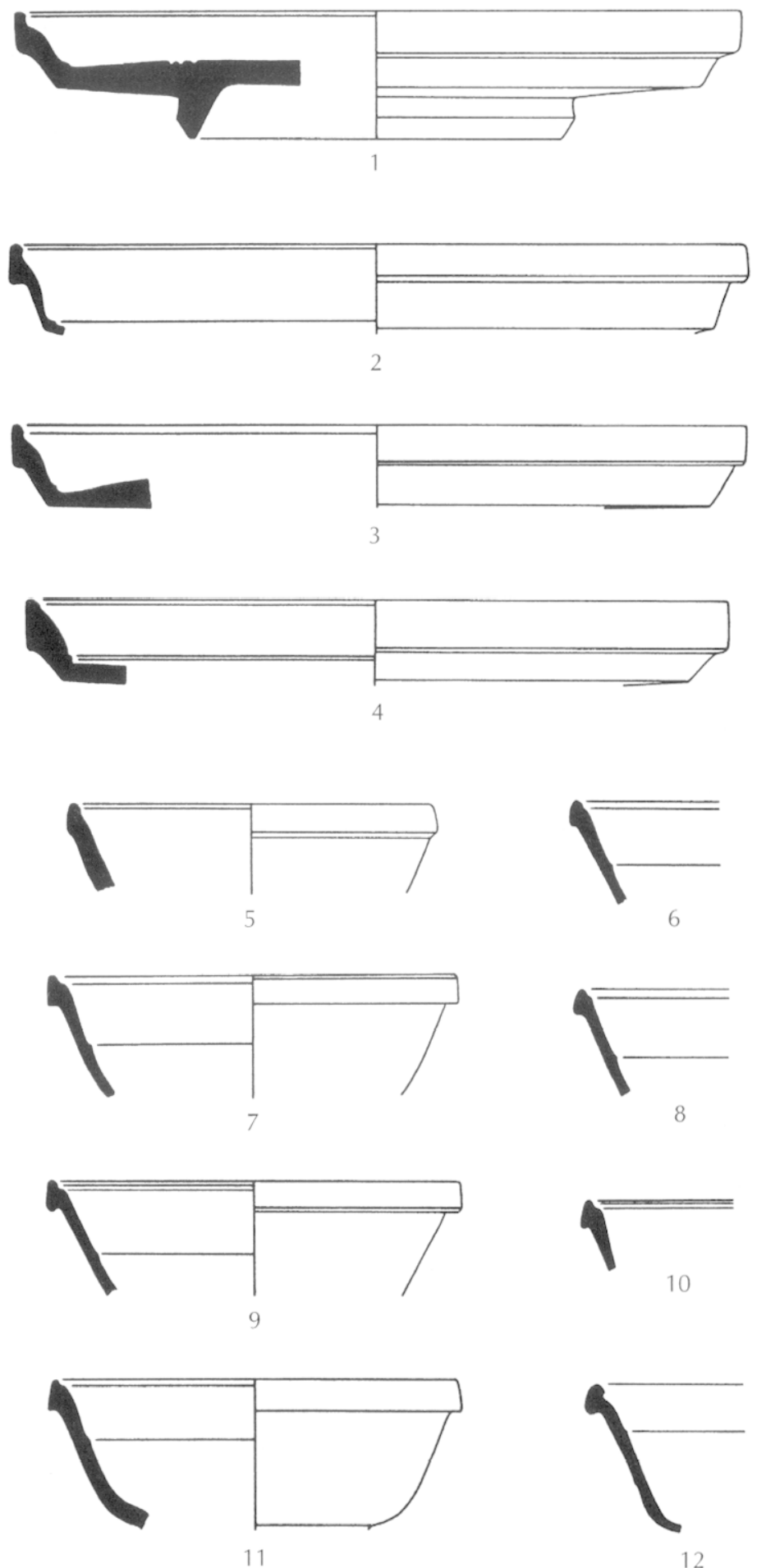
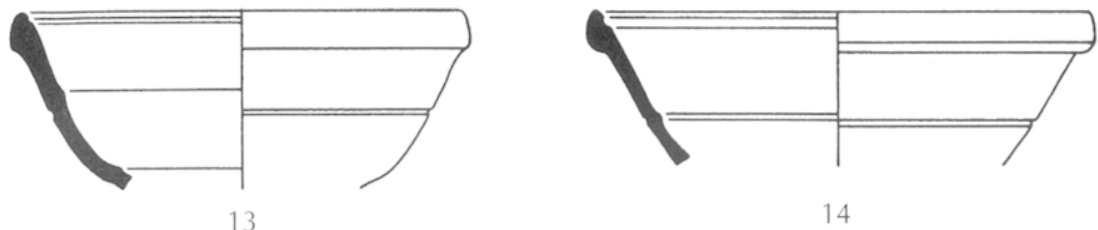

14

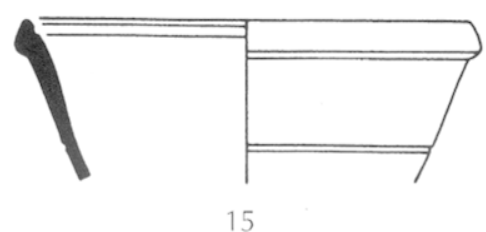

Pl. 10. Einsemble I, sigillee, service IB : assiettes du groupe 2 (1-4), bols du groupe 1 (5-15) (échelle : 1/2). 
FRAN

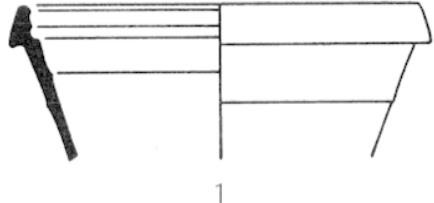

PI. 11. ENSEMBLE I

Bols du service IB, groupes 2 ( 85 ex.) et 3 (210 ex.)

1-6, groupe $\mathbf{2}$ : lèvres courtes et nettement rentrantes de section triangulaire, à face interne concave; attache bord/fond de profil arrondi ; parois interne et externe divisées en deux parties le plus souvent inégales et variant encore sensiblement d'un exemplaire à l'autre.

7-15, groupe 3 : lèvres de hauteur moyenne peu profilées; paroi interne non divisée ou à division bipartite, paroi externe à division bipartite variable.

Ettlinger, 1983, pl. 31, n० 13, 15, 19; Genin, 1993, fig. $16, n^{\circ} 4$ et fig. $20, n^{\circ} 88-89$.
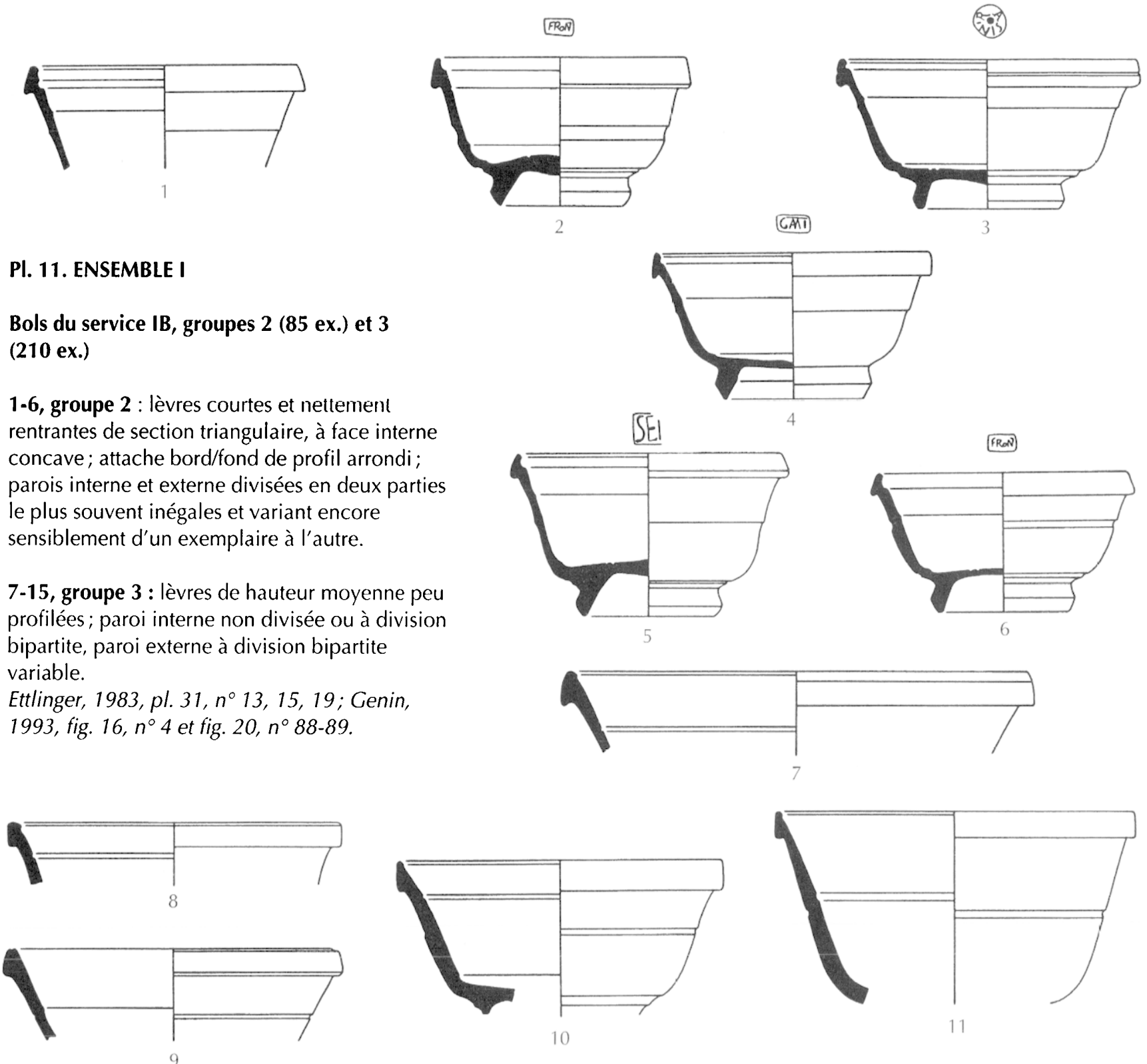

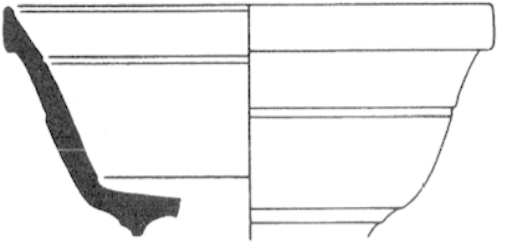

10

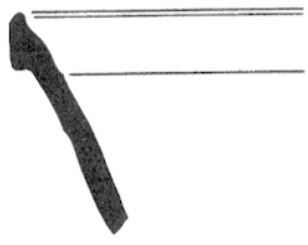

13

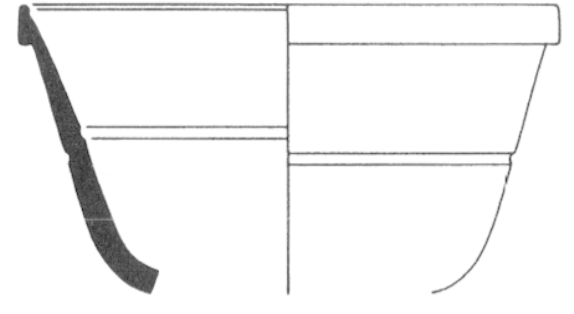

11

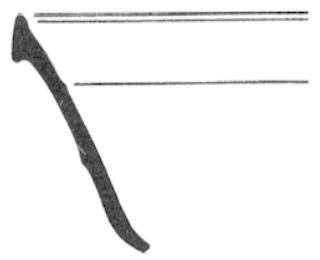

14

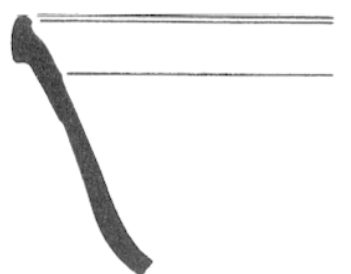

15

PI. 11. Ensemble I, sigillée, service IB : bols du groupe 2 (1-6) el du groupe 3 (7-15) (échelle : 1/2). 


\section{PI. 12. ENSEMBLE I}

\section{Bols du service IB, groupes 4 (2 274 ex.) et 5} (1 592 ex.)

1-7, groupe 4, "bols type Lyon " : les lèvres, hautes et fines, sont situées dans le prolongement exact de la paroi, formant ainsi des embouchures très évasées ; elles sont toujours dégagées à leur extrémité supérieure par un bourrelet doublé ou non d'une rainure; les parois interne et externe sont dans la plupart des cas divisées en deux parties sensiblement égales.

Ce type de bols IB est sans aucun doute le plus répandu sur le limes à l'époque augustéenne : totalement absent à Bolsena, bien attesté à Neuss, il constitue à Haltern la quasi-totalité des bols du service IB. II représente ici plus de la moitié des bols du service IB de la première période de production. Ettlinger, 1983, pl. 32, n० 1-11; von Schnurbein, 1982, pl. 4-5 et pl. 6, n० 77-80; Genin, 1993, fig. 20, $n^{\circ} 85-87$.

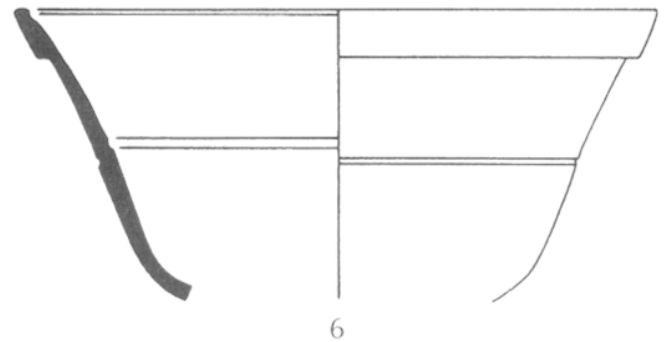

8-12, groupe 5 : proches des bols du groupe 4, ils s'en distinguent néanmoins par des traits morphologiques plus variés : les lèvres se détachent de la paroi, sont soulignées par un bourrelet suivi d'une gorge très prononcée et offrent des profils moins évasés; la division bipartite des parois interne et externe est, quant à elle, moins régulière.

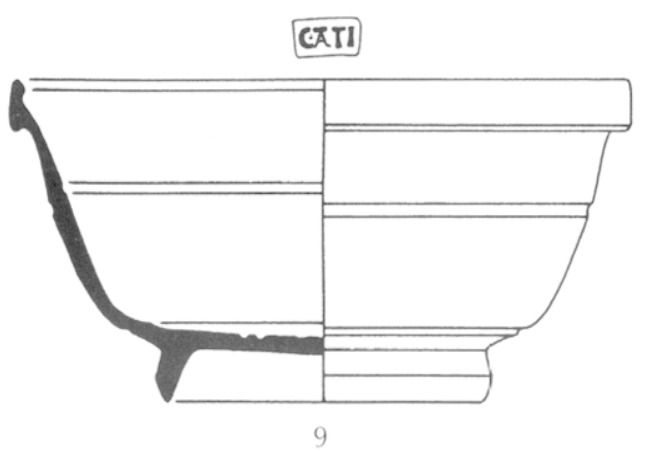

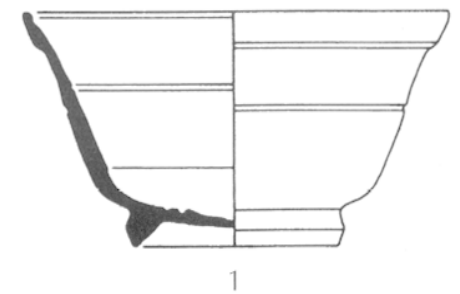
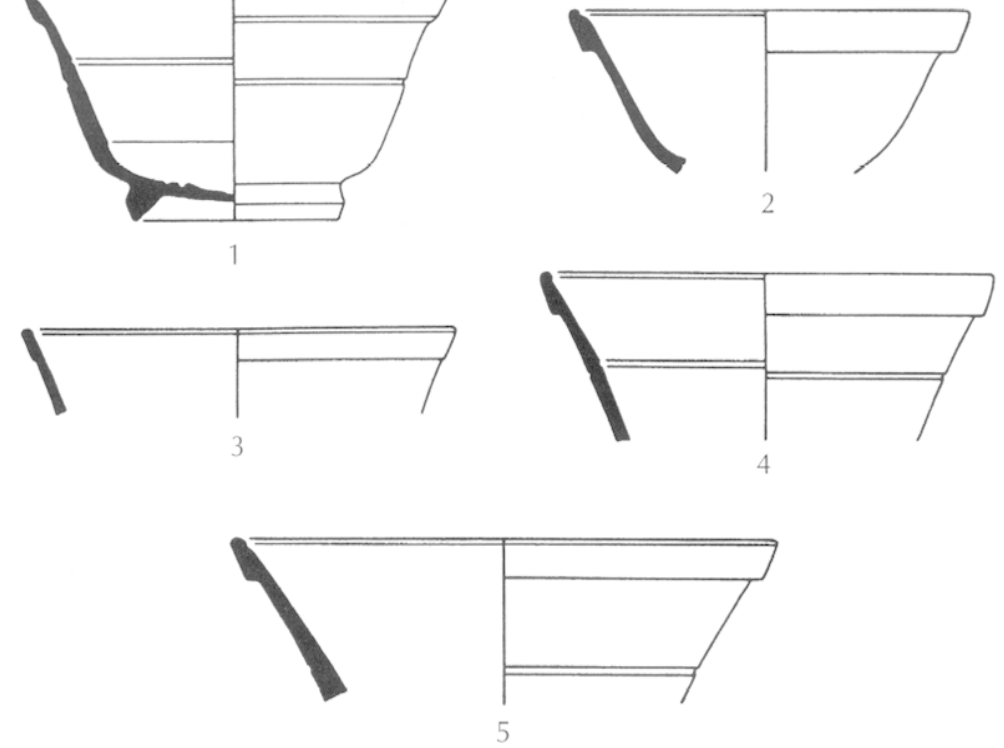

HQR
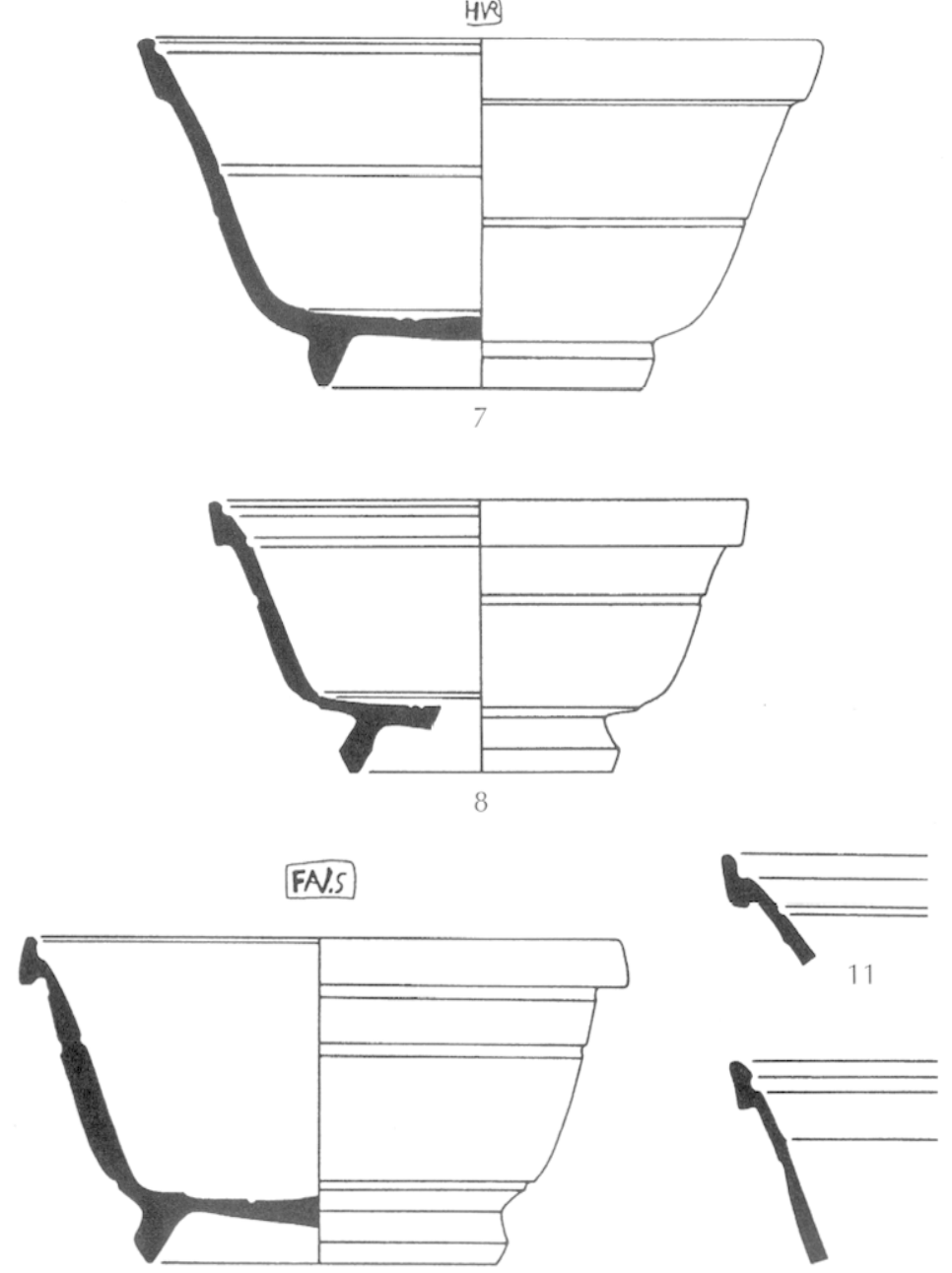

10

Pl. 12. Ensemble I, sigillée, service IB. : bols du groupe 4 (1-7) et du groupe 5 (8-12) (échelle: 1/2). 


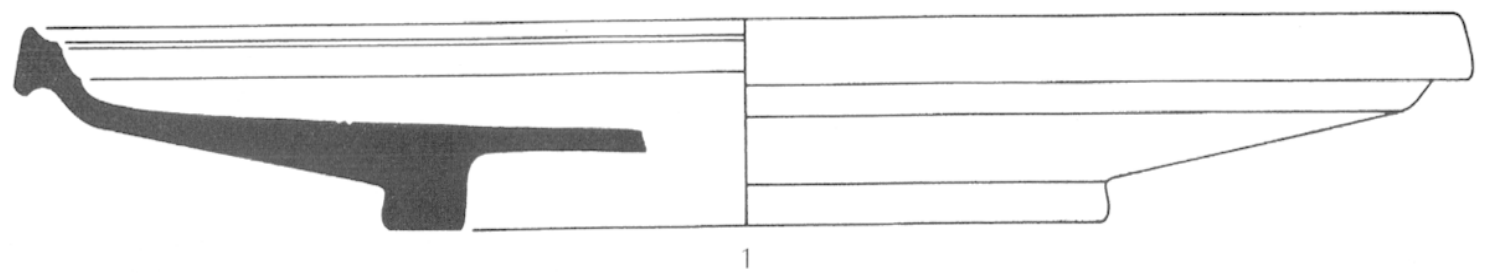

\section{PI. 13. ENSEMBLE I}

\section{SERVICE IB/IC (2 479 ex.)}

1-3, plats IB/IC (32 ex.) : groupe peu connu. Les lèvres sont rectilignes, de hauteur moyenne, nettement séparées de la paroi et régulièrement dégagées par un léger ressaut sur leur face interne. Division interne bipartite dans la majeure partie des cas. On retrouve quelques formes proches à Neuss, formes d'ailleurs classées en "service I type Lyon ". Ces profils évoquent également la variante a du type Goudineau 17 (Goudineau, 1968).

Ettlinger, 1983, pl. 4, n²2-29 et pl. 17, n० 17 (plat " service I type Lyon ").

4-6, assiettes IB/IC (1 757 ex.) : elles montrent comme les plats IB/IC une certaine homogénéité morphologique : les lèvres sont en général droites, de hauteur moyenne, et systématiquement soulignées à l'intérieur par un léger ressaut ; la paroi interne tend à adopter une division tripartite classique. Ce groupe intermédiaire entre le service IB et le service IC est totalement absent à Haltern, faiblement représenté à Neuss où ii est défini comme " service I type Lyon " (cf. supra). Ettlinger, 1983, pl. 17, n०21-22.

7-9, bols IB/IC (690 ex.) : les bols IB/IC montrent des profils similaires. Les lèvres, de hauteur moyenne, sont régulièrement dégagées par un léger ressaut et prolongées par des panses à division interne et externe bipartite. Les exemplaires répertoriés représentent deux calibres bien distincts : sur les bols de petits diamètres, la panse offre un profil arrondi et sur les bols de grands diamètres, un profil oblique ou légèrement incurvé. On ne peut proposer de parallèles précis pour ce groupe qui rassemble tout de même plus de $11 \%$ de l'ensemble des bols du service I de l'ensemble I.
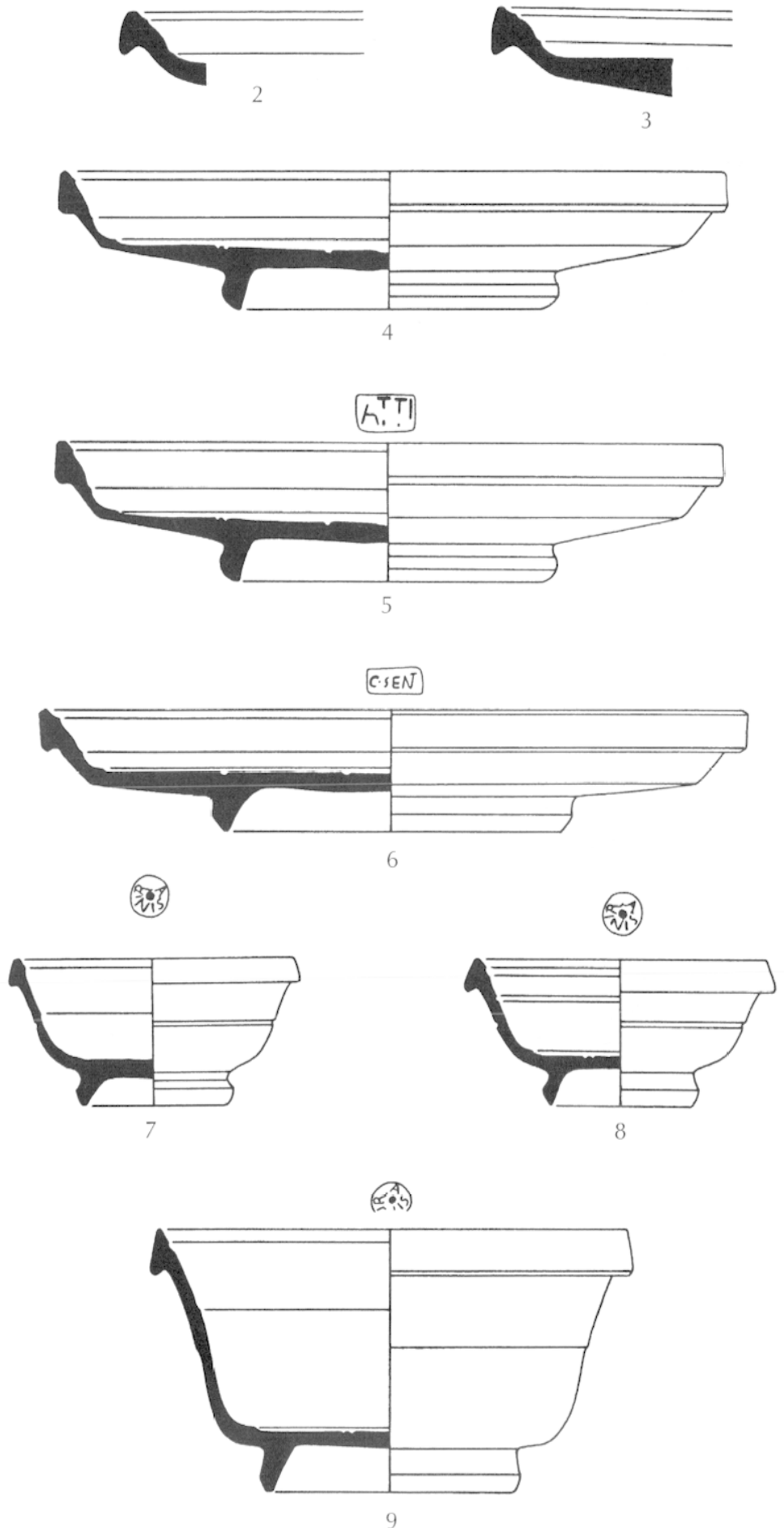

Pl. 13. Ensemble I, sigillée, service IB/IC : plats (1-3), assietles (4-6) et bols (7-9) (échelle : $1 / 2$ ). 


\section{PI. 14. ENSEMBLE I}

\section{PLATS DU SERVICE IC, groupes 1 (11 ex.) et 2} (72 ex.)

1-6, groupe 1 : lèvres de section triangulaire, très nettement détachées de la paroi, division interne tripartite, attache bord/fond marquée par un angle vif. II s'agit d'une série peu fréquente pour laquelle on ne trouve qu'un parallèle approchant à Haltern et quelques profils d'assiettes à Neuss classés en "service I type Lyon ". von Schnurbein, 1982, pl. 8, 95 ; Ettlinger, 1983, pl. 17, 3-9.

7-15, groupe 2 : lèvres épaisses, collées à la paroi, dégagées par un fin sillon; division interne tripartite régulière, attache bord/fond de profil arrondi.

Goudineau, 1968, type 17 b et $c$; von Schnurbein, 1982, pl. 7-9-11; Ettlinger, 1983, pl. $6, n^{\circ} 1-4$; Schindler, Scheffenegger, 1977, pl. 22, n० 5-6.

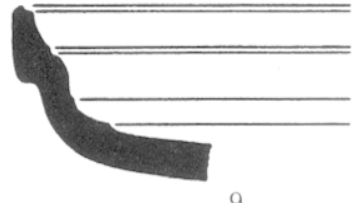

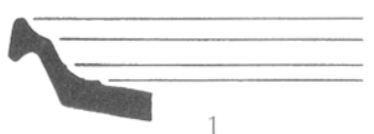
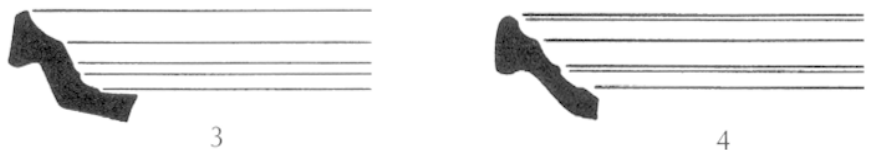

4
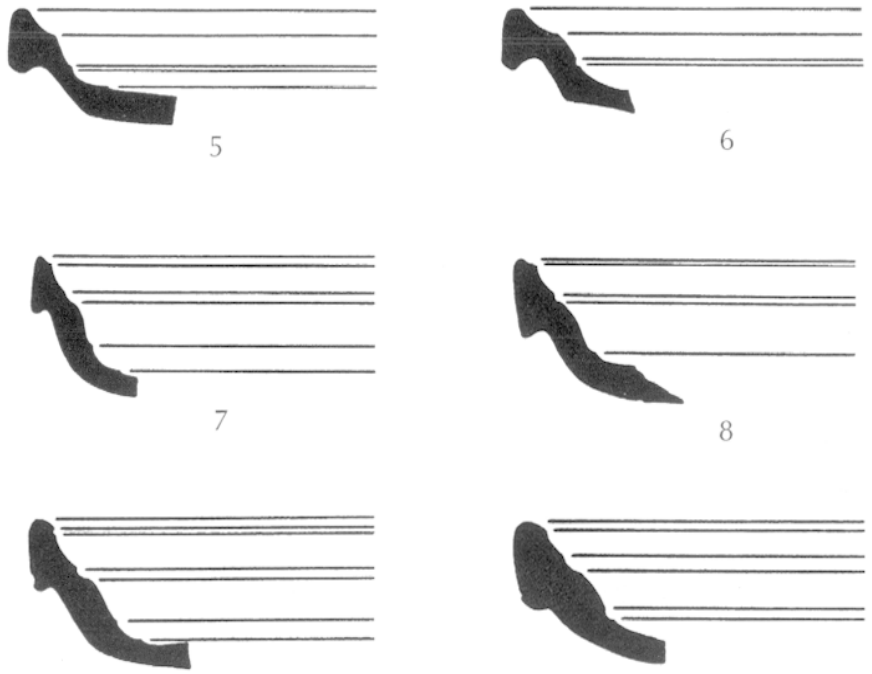

10

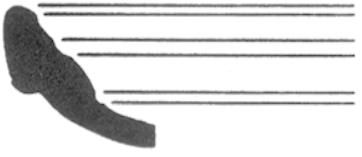

11
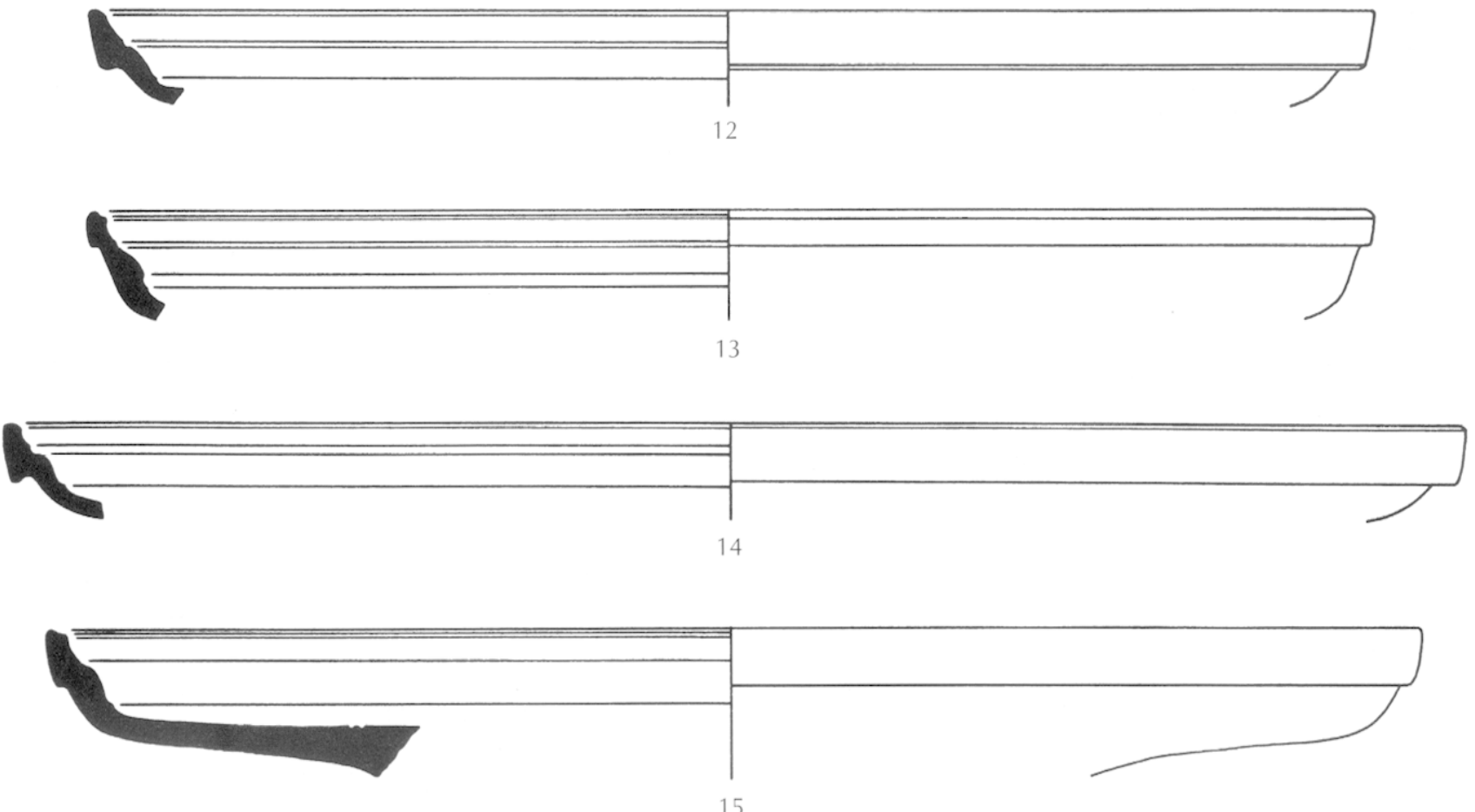

Pl. 14. Ensemble I, sigillée, service IC : plats du groupe 1 (1-6) et du groupe 2 (7-15) (échelle: 1/2). 


\section{PI. 15. ENSEMBLE I}

\section{ASSIETTES DU SERVICE IC (2 340 ex.)}

L'étude révèle la présence de cinq groupes ou « variantes » d'homogénéité et d'importance numérique inégales.

Groupe 1 : 986 ex. $(42 \%)$

Groupe $2: 251$ ex. (11\%)

Groupe $3: 825$ ex. $(35 \%)$

Groupe $4: 44$ ex. (2\%)

Groupe $5: 234$ ex. $(10 \%)$

Assiettes du service IC, groupe 1, profils précoces (série 1) et formes rares (série 2)

Le groupe 1 apparaît comme un conglomérat de formes peu standardisées au sein desquelles on peut sommairement distinguer deux grandes séries.

On peut signaler pour la série 1 quelques similitudes avec des profils de Neuss; en revanche la série 2 n'existe pas plus à Neuss que sur d'autres sites du limes.

1-14, série 1 : lèvres courtes nettement éversées à face interne concave; division tripartite interne irrégulière.

Ettlinger, 1983, pl. 13, n' 17-25.

$\mathbf{1 5 - 2 2}$, série 2 : lèvres plus hautes tendant à la verticale, division interne tripartite irrégulièrement marquée par plusieurs stries ou gorges.
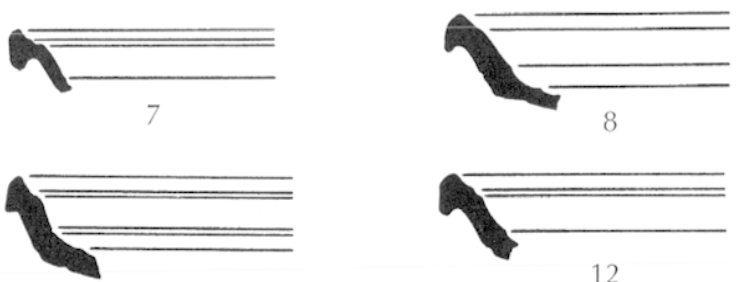

12

C.SER
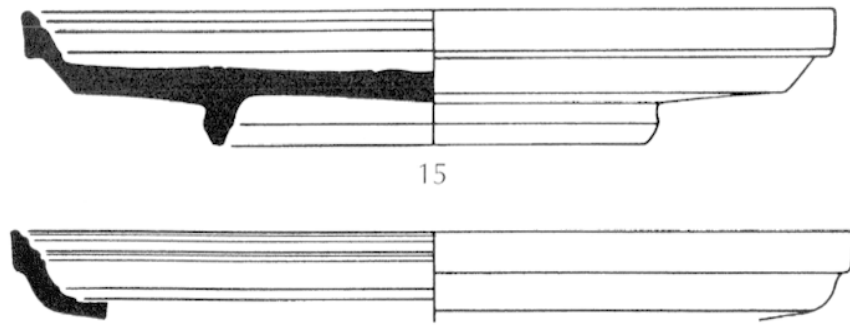

17

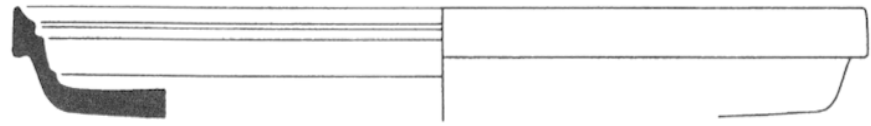

20
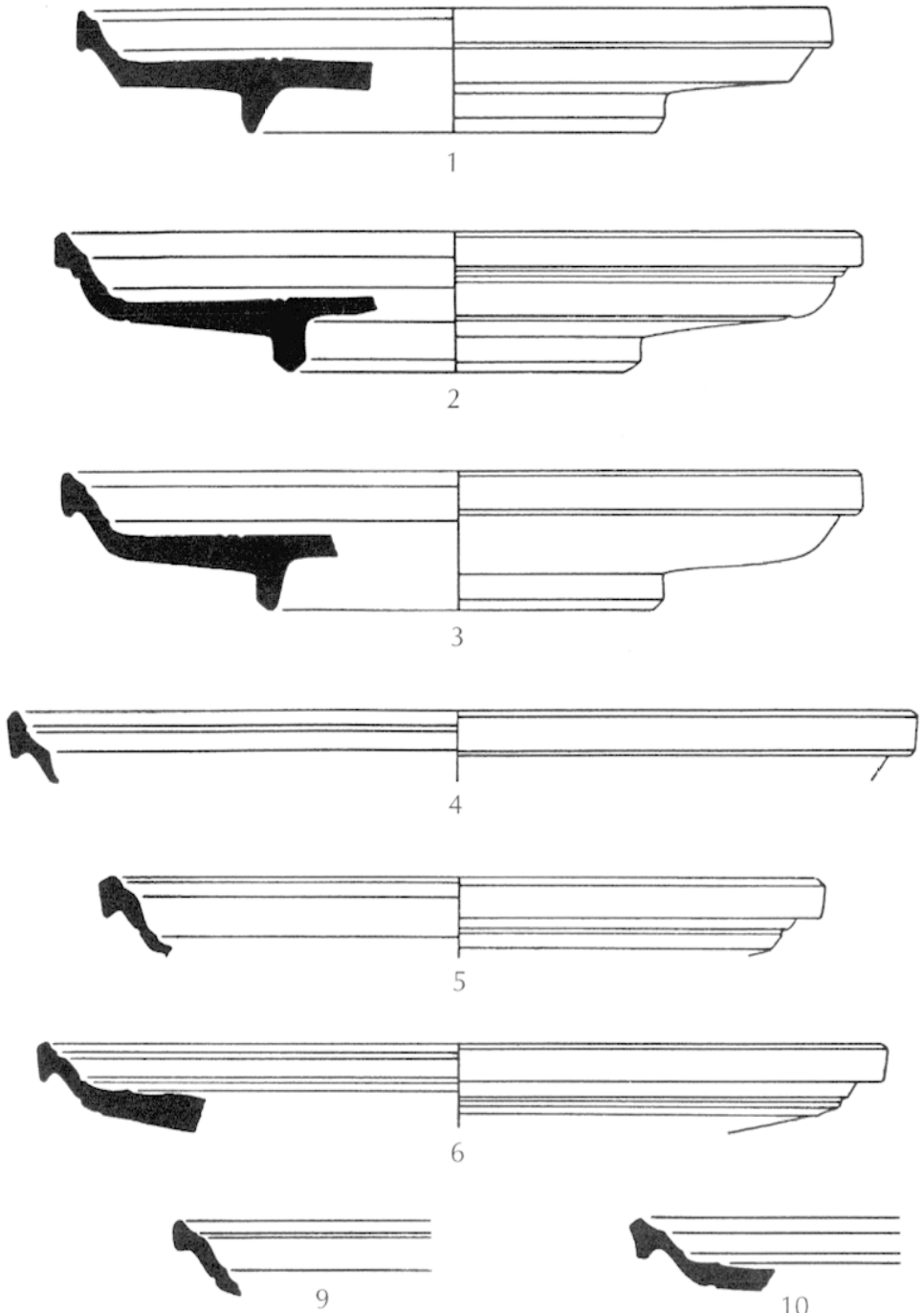

10
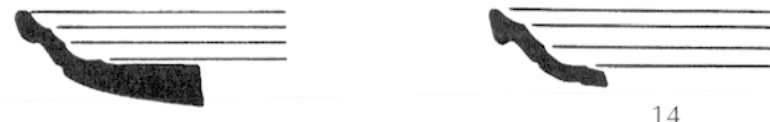

14

SEN

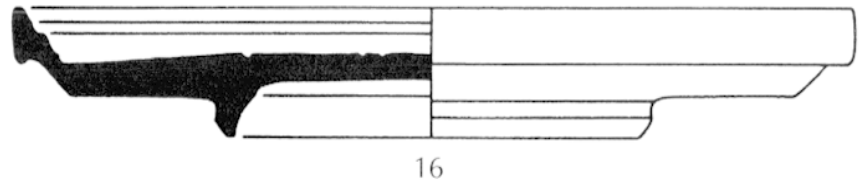

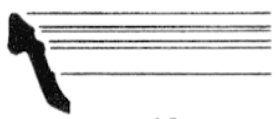

18

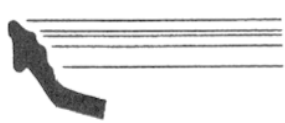

21

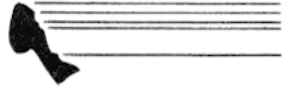

19

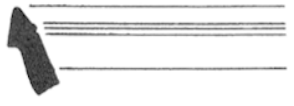

22

Pl. 15. Ensemble I, sigillée, service IC : assiettes du groupe 1 (échelle: $1 / 2$ ). 


\section{PI. 16. ENSEMBLE I}

\section{Assiettes du service IC, groupes 2 (251 ex.) et 3 (825 ex.)}

1-17, groupe 2 : il offre en revanche une image beaucoup plus homogène. Les lèvres sont droites, peu séparées de la paroi et forment une embouchure évasée; la division tripartite interne reste irrégulière.

18-23, groupe 3 : lèvres verticales de hauteur moyenne, division bipartite interne régulière; division tripartite soulignée par un renflement. L'attache bord/fond marquée généralement par un angle vif adopte dans quelques cas un profil arrondi. Quelques exemplaires rappellent une forme attestée à Dangstetten (Fingerlin, 1986, 320-25).
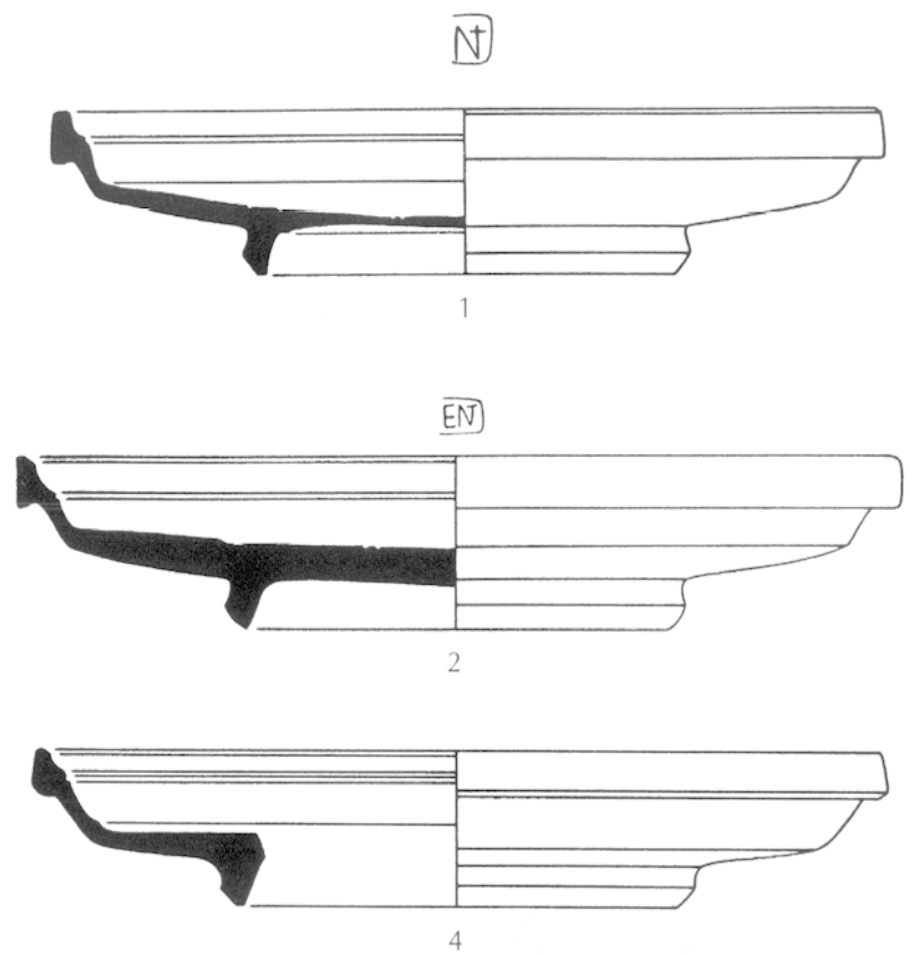
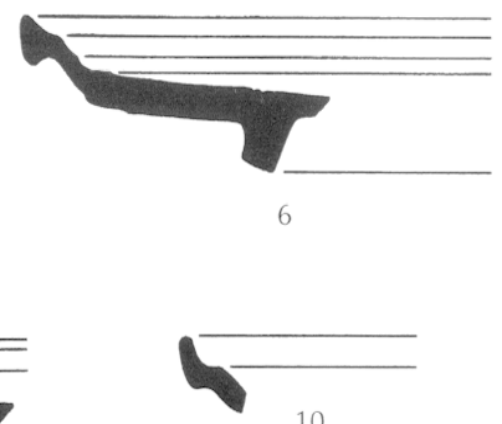

10

9

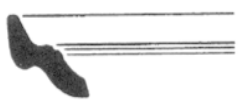

14

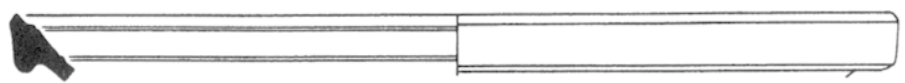

18

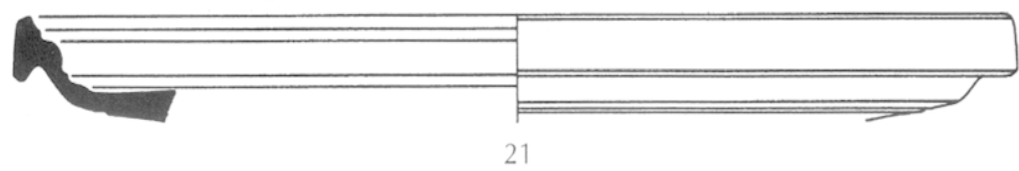

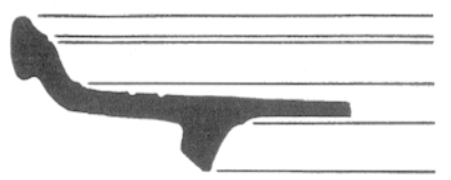

7

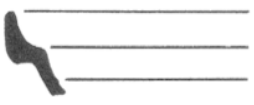

11

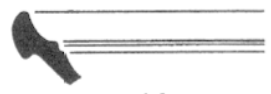

16

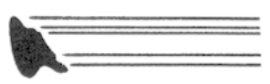

19

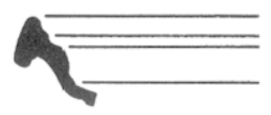

22

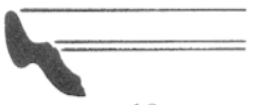

12
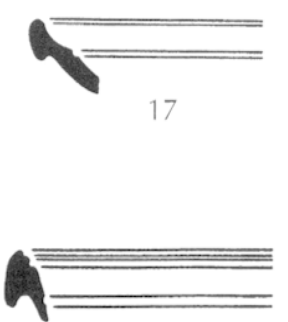

20

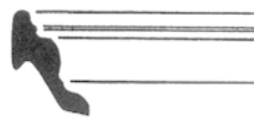

23

Pl. 16. Ensemble I, sigillée, service IC: assiettes du groupe 2 (1-17) et du groupe 3 (18-23) (échelle : 1/2). 


\section{PI. 17. ENSEMBLE I}

\section{Assiettes du service IC, groupe 4 (44 ex.) et 5 (234 ex.)}

1-7, groupe 4 : profils dénotant une évolution typologique vers les formes classiques du service IC. Les 44 exemplaires se caractérisent par des bords légèrement arrondis à face interne concave évoquant le type Goudineau 23. On observe que la division interne de la paroi n'obéit pas encore de façon systématique et régulière au schéma bipartite connu sur les assiettes de ce type.

Goudineau, 1968, type 23; von Schnurbein, 1982, pl. 21, $n^{\circ} 227-229 ;$ Ettlinger, 1983, pl. 16, n 1-13.

ATID

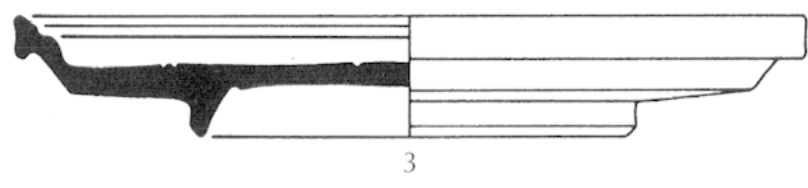

AITD

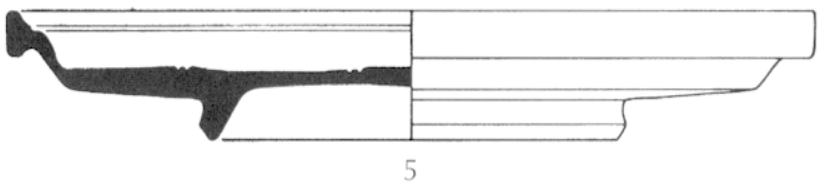

8-19, groupe 5 : ces assiettes présentent des profils parfaitement classiques : lèvres droites ou évasées, peu éversées et prolongées par une paroi parfaitement oblique se raccordant au fond par un angle vif; la division tripartite interne de la paroi est systématique et régulière. Le groupe 5 correspond au type d'assiettes IC le plus abondant à Haltern, type globalement très homogène en dépit de la présence de légères variantes. On note que ces profils classiques, en l'occurrence absents à Neuss, ne représentent que $10 \%$ des assiettes IC de la première période de production lyonnaise. Goudineau, 1968, type 17, variantes b et c; von Schnurbein, 1982, pl. 16, n० 159-170 et pl. 19-20; Ettlinger, 1983, pl. 17, $n^{\circ} 27$ (" service I type Lyon »); Fingerlin, 1986, 52-20; Genin, 1993, fig. 19, n 66-81.

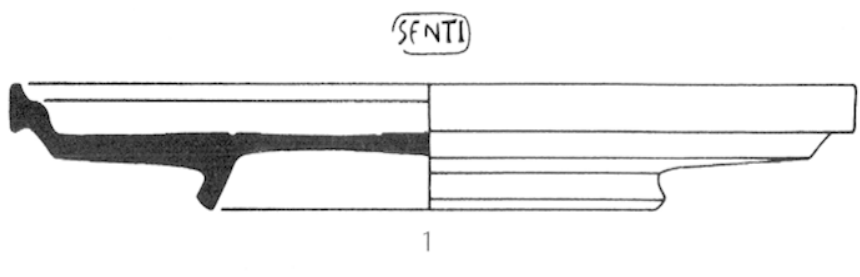

(ATTI)
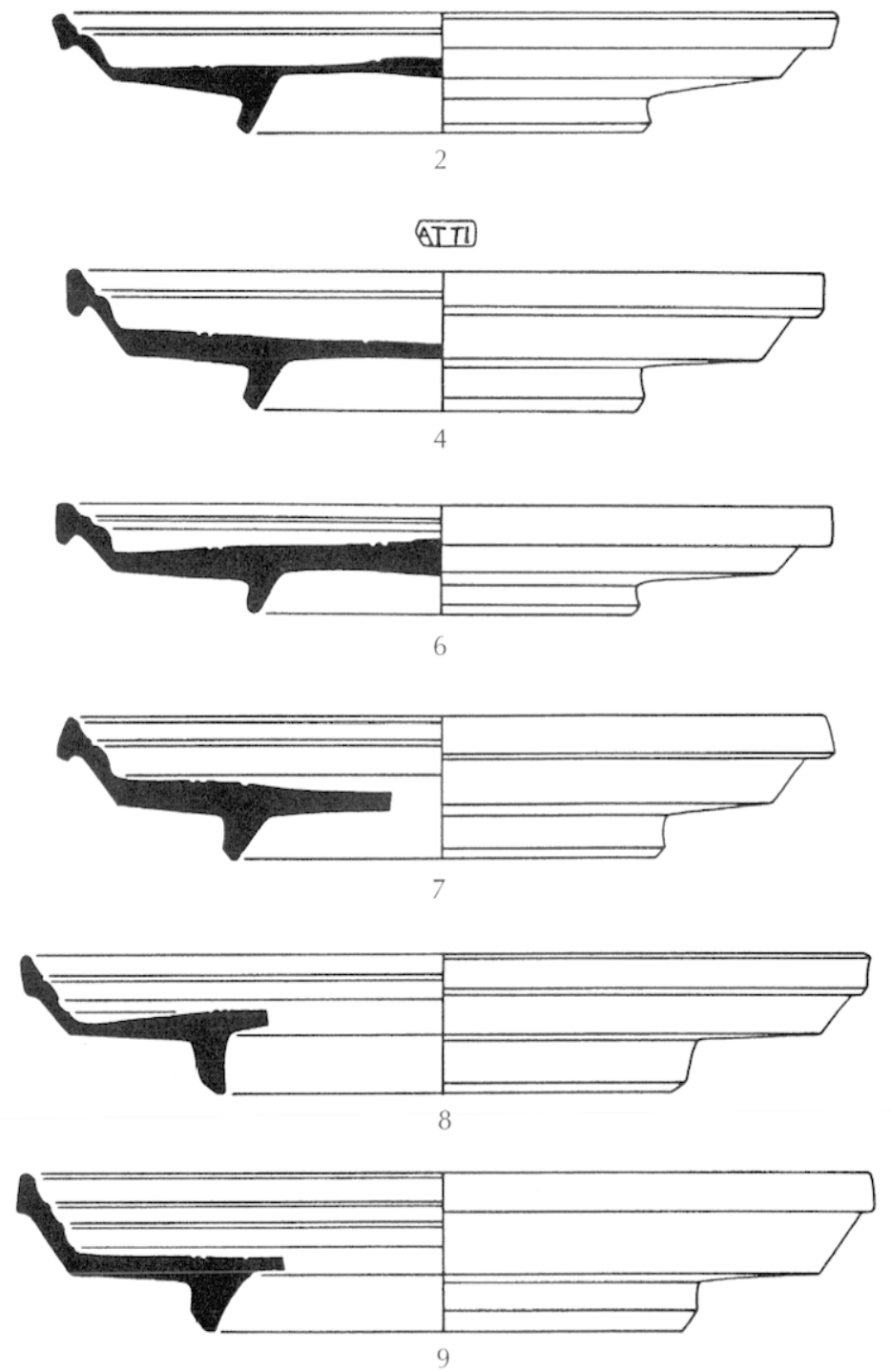

9

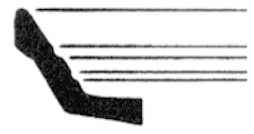

13

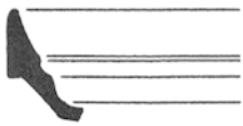

18

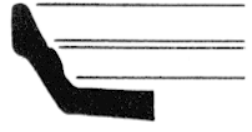

14

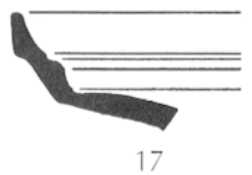

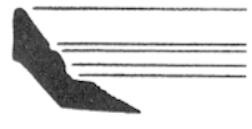

19

Pl. 17. Ensemble I, sigillée, service IC : assiettes du groupe 4 (1-7) et du groupe 5 (8-19) (échelle: 1/2) 


\section{PI. 18. ENSEMBLE I}

\section{BOLS DU SERVICE IC (1 016 ex.)}

Ils déterminent quatre groupes différents. Les groupes 1 et 2 montrent deux types précoces de ce service et les groupes 3 et 4 les variantes classiques les plus abondamment représentées à l'époque augustéenne.

Groupe $1: 88$ ex. $(8,5 \%)$

Groupe $2: 162$ ex. $(16 \%)$

Groupe $3: 19$ ex. $(2 \%)$

Groupe $4: 747$ ex. $(73,5 \%)$.

1-8, groupe 1 : bols, pour la plupart de petits diamètres; bords arrondis à face interne concave, division interne bipartite irrégulière, division externe variable; attache bord/fond de profil arrondi.

Ce groupe ne correspond pas à un type bien défini; on relève néanmoins quelques similitudes avec des bols de Haltern. von Schnurbein, 1982, pl. 27, n० 464-469 et pl. $28, n^{\circ} 474$.

\section{9-16, groupe 2 : lèvres courtes de section} triangulaire, nettement détachées du col ; paroi rectiligne à division interne et externe bipartite. Ces bols rappellent les types italiques Goudineau 18 et 24 . On ne retrouve pas cependant de parallèles satisfaisants à Neuss ou à Haltern ; quelques bols isolés de Dangstetten présentent des profils proches.

Fingerlin, 1986, 364-5.
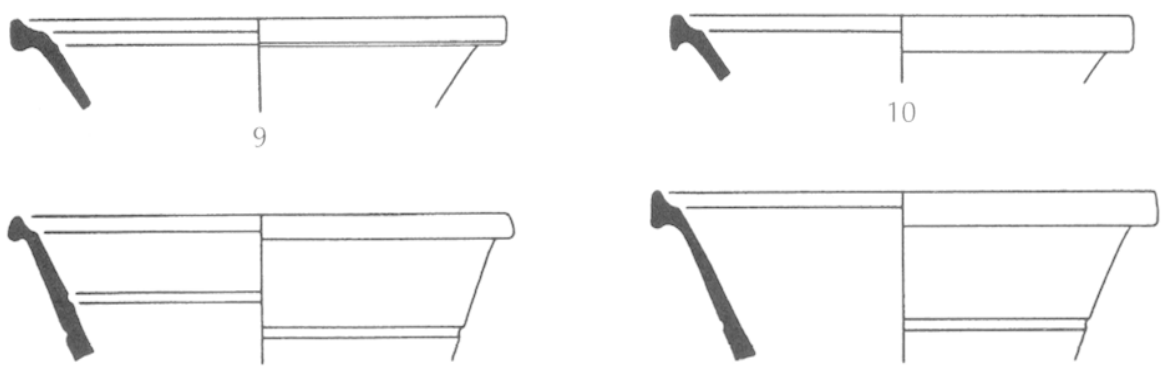

12

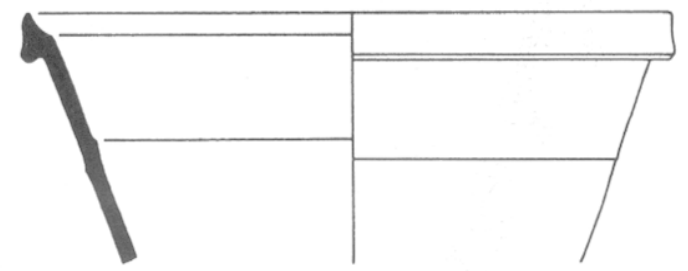

15
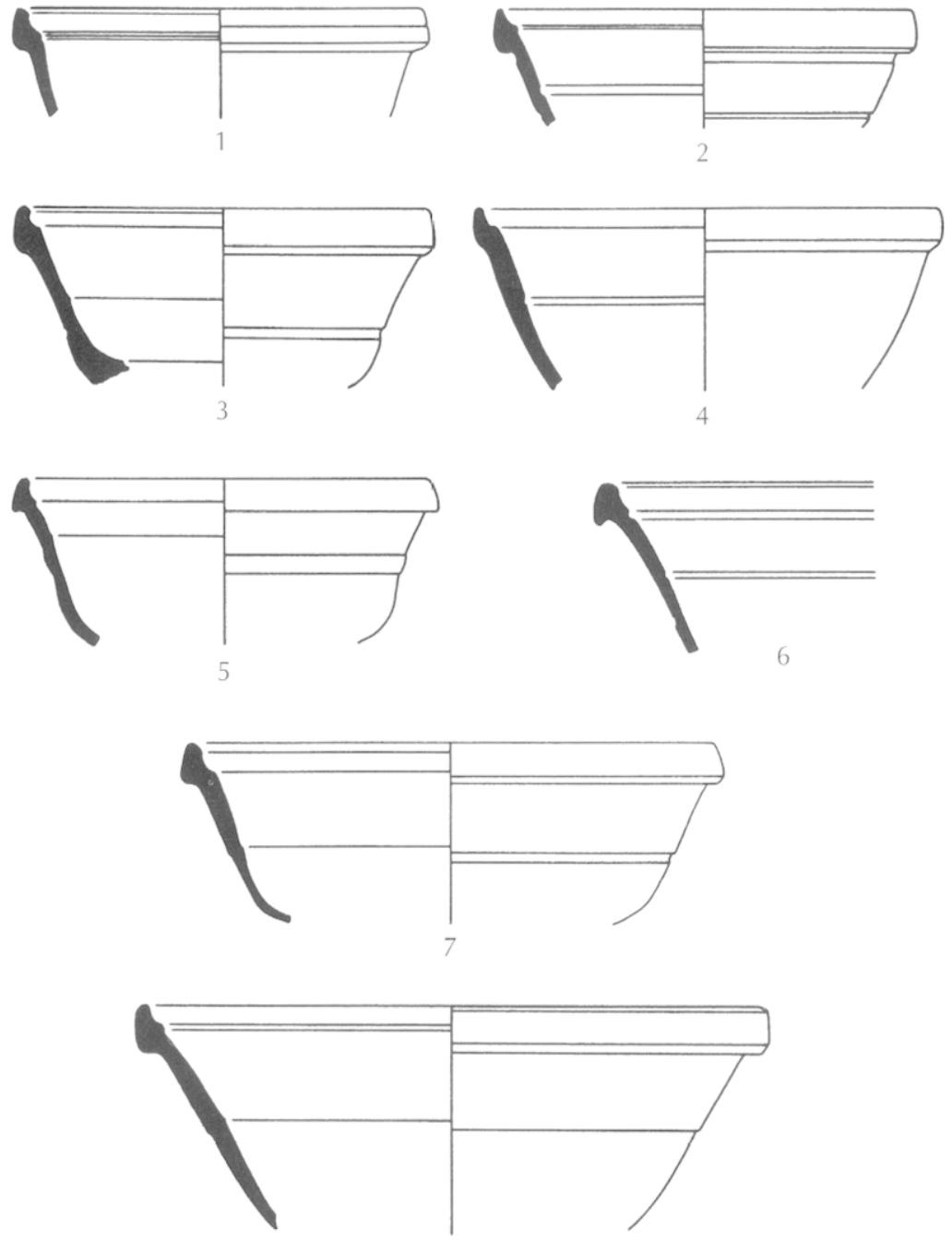

8
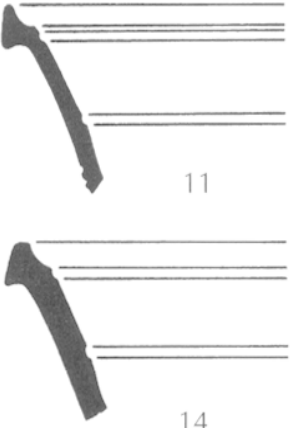

14

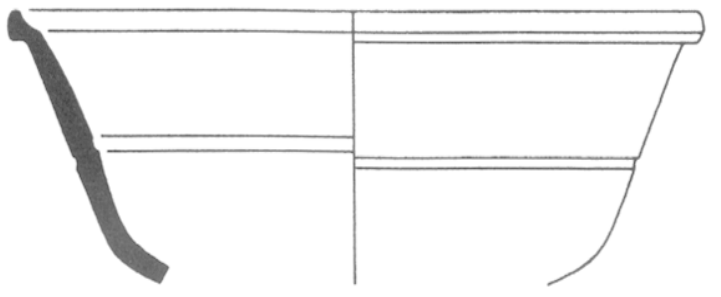

16

Pl. 18. Ensemble I, sigillée, service IC : bols du groupe 1 (1-8) et du groupe 2 (9-16) (échelle : 1/2). 


\section{PI. 19. ENSEMBLE I}

Bols du service IC, groupes 3 (19 ex.) et 4 (747 ex.)

1-4, groupe 3 : lèvres hautes et fines situées dans le prolongement exact de la paroi et formant des embouchures très évasées; la division interne des lèvres se compose $d^{\prime}$ 'une gorge haute et peu prononcée suivie d'un ou plusieurs sillons. Ce type semble peu abondant à Neuss et à l'inverse bien représenté à Haltern où figurent des profils extrêmement proches.

Ettlinger, 1983, pl. 37, $n^{\circ} 11$; von Schnurbein, 1982, pl. 23, n०394-396b et pl. 24, n०396c;

Genin, 1993, fig. 20, n 95.

5-10, groupe 4 : lèvres droites ou légèrement obliques dégagées par une large gorge ; parois rectilignes à division interne et externe bipartite. Ce groupe comprend les profils les plus classiques de bols du service IC. On relève un grand nombre de parallèles sur les principaux sites de référence. Ettlinger, 1983, pl. 35-36-37; von Schnurbein, 1982, pl. 23, n०382-389; Genin, 1993, fig. 20, n०91-93.
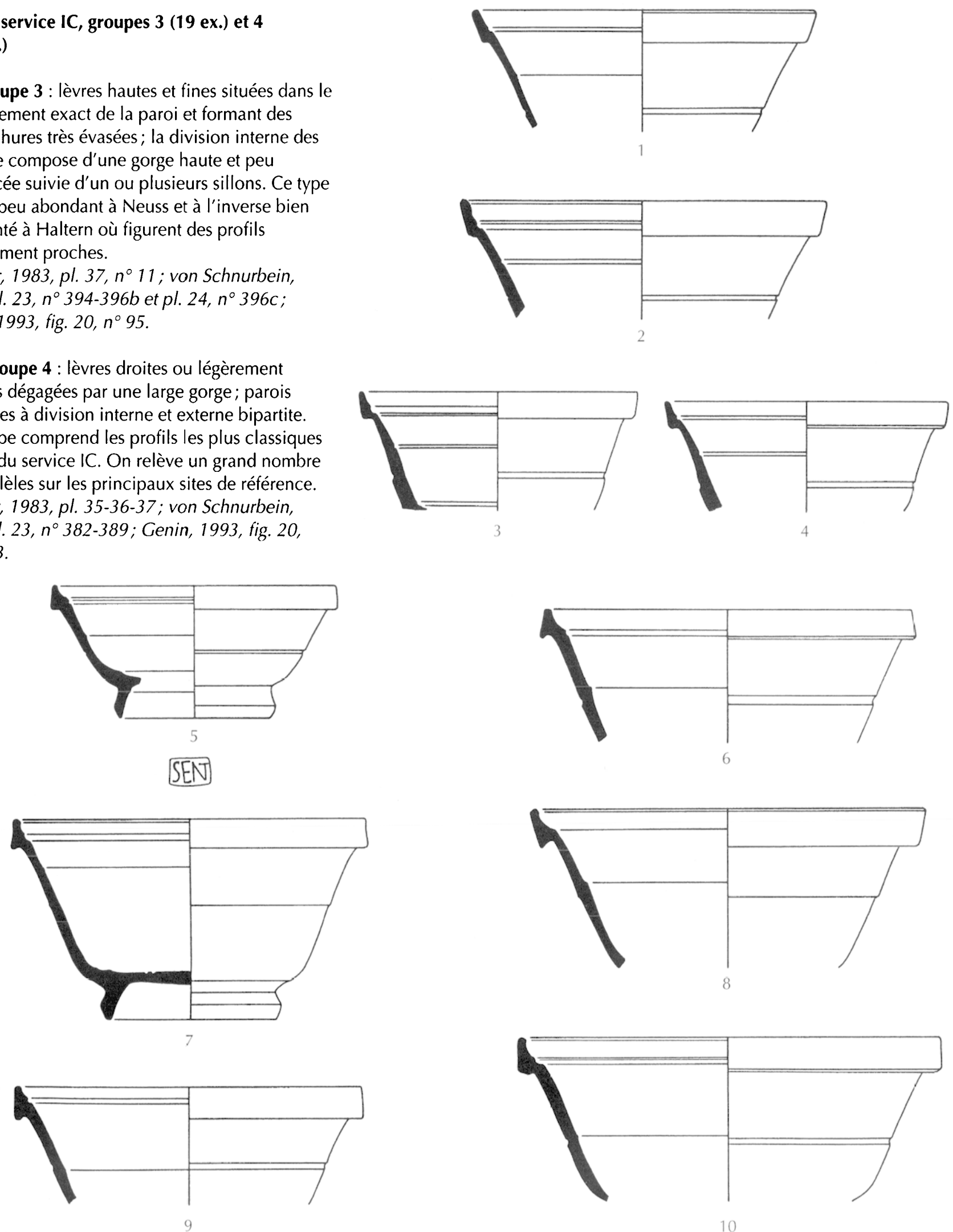

P1. 19. Ensemble I, sigillée, service IC : bols du groupe 3 (I-4) et du groupe 4 (5-10) (échelle : 1/2). 


\section{PI. 20. ENSEMBLE I}

\section{SERVICE II : assiettes groupes 1 et 2 , bols groupe 1}

1-7, assiettes du service II (225 ex.) : elles appartiennent, à l'exception de deux d'entre elles, à un seul et même type caractérisé par des bords incurvés non profilés, quelquefois rainurés à leur extrémité supérieure (1-5). Ce type totalement absent à Haltern s'avère de façon générale rarissime sous Auguste. On connaît seulement quelques exemplaires isolés à Neuss et à Dangstetten. Deux assiettes (6-7) offrent des profils assimilables aux formes classiques du service II. 1-9: Ettlinger, 1983, pl. 18, n 1, 5, 6; Fingerlin, 1986, 356-18.
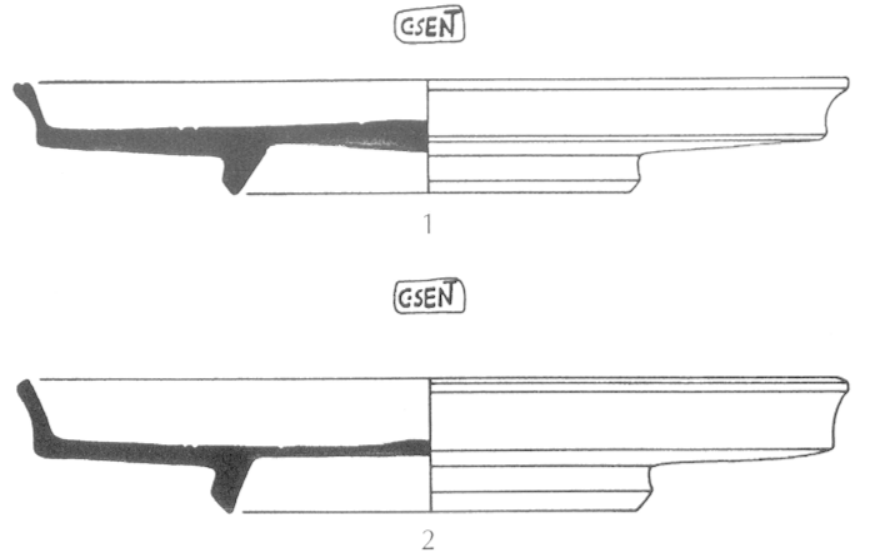

CSEN)
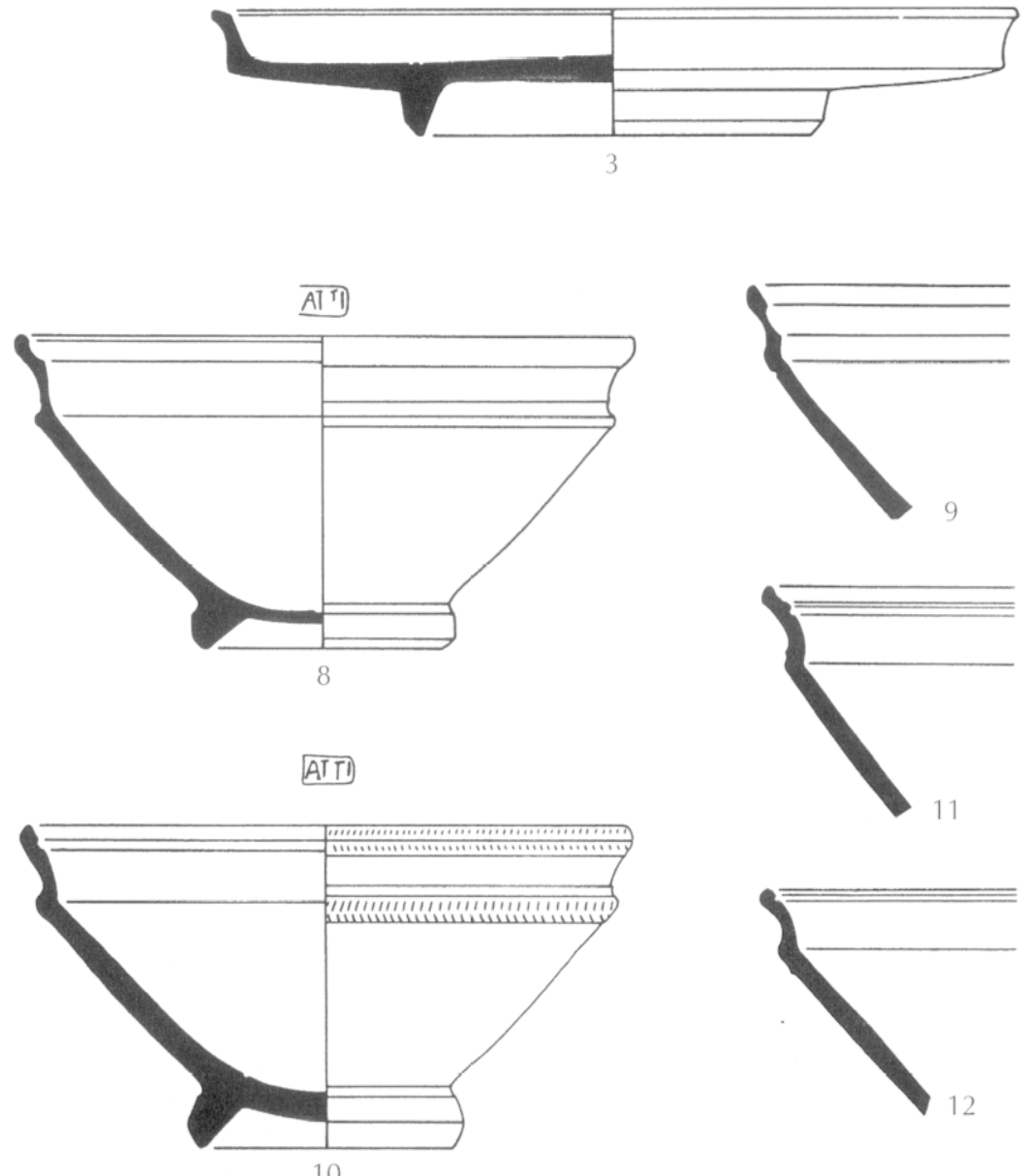
diamètre d'ouverture est dans la plupart des cas deux fois supérieur à la hauteur du bol. L'attache bord/panse est marquée par un angle vif. Noter que les guillochis quasi systématiques sur les bols du service II s'avèrent plus rares dans ce groupe qui comprend de nombreux bords lisses.

Cette variante originale n'est pas attestée sur les principaux sites de référence; on relève un seul parallèle approchant dans le mobilier d'Oberaden. Elle constitue ici $61 \%$ des bols du service II du premier ensemble.

Albrecht, 1942, pl. 26, n०18.
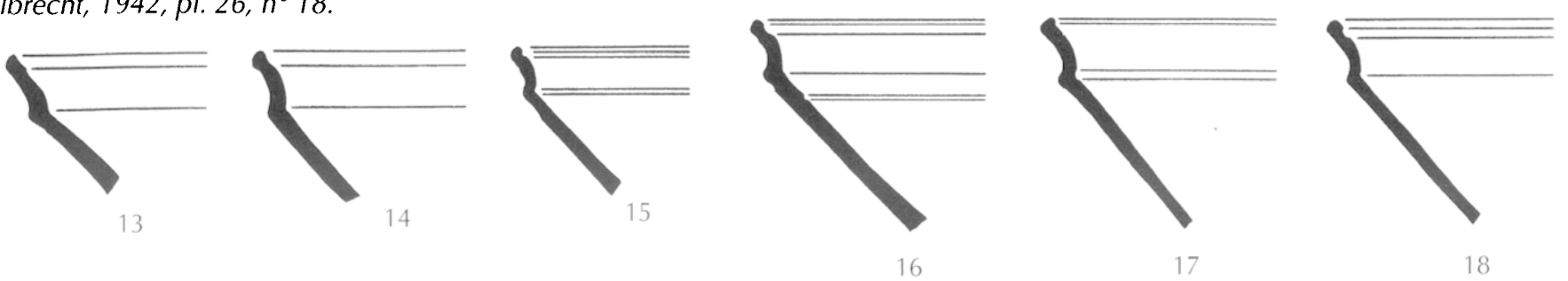

P1. 20. Ensemble I, sigillée, service II : assiettes du groupe 1 (1-5) et du groupe 2 (6-7), et bols du groupe 1 (8-18). (échelle: 1/2). 


\section{PI. 21. ENSEMBLE I}

Bols du service II, groupes 2 (47 ex.) et 3 (113 ex.)

1-3, groupe 2 : bords droits délimités par des bourrelets guillochés parfaitement réguliers et prolongés par des parois rectilignes. Ce groupe est bien homogène tant sur le plan typologique que morphologique : il s'agit, à de rares exceptions près, de bols de petits diamètres, et portant de plus, pour les exemplaires archéologiquement complets, le même timbre de potier (T (ITIVS) M (ALVS)

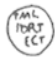

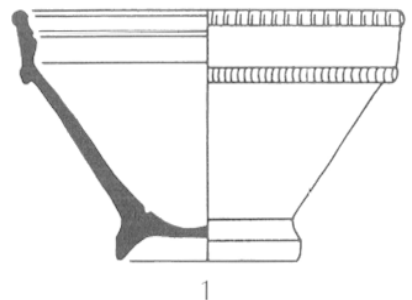

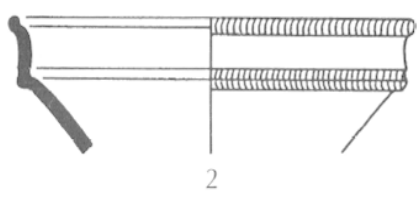

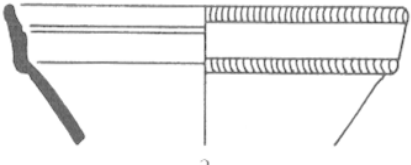

F (ORTVNATVS) FECIT. Ce type est connu sous Auguste, en particulier à Haltern où les exemplaires publiés portent la même signature. II ne représente ici que $5 \%$ des bols du service $I I$.

von Schnurbein, 1982, pl. 49, $n^{\circ}$ 603-605; Ettlinger, 1983, pl. 42, n० 17; Genin, 1993, fig. 21, n 163-166.

4-13, groupe 3 : lèvres hautes à face externe arrondie ou oblique, soulignées à l'intérieur par une gorge large et très prononcée; attache bord/panse de profil arrondi. La division externe des bords ne suit pas un schéma uniforme. Cette variante, inconnue sur le limes comme sur les sites lyonnais, rassemble pourtant $12 \%$ des bols du service II de l'ensemble I.
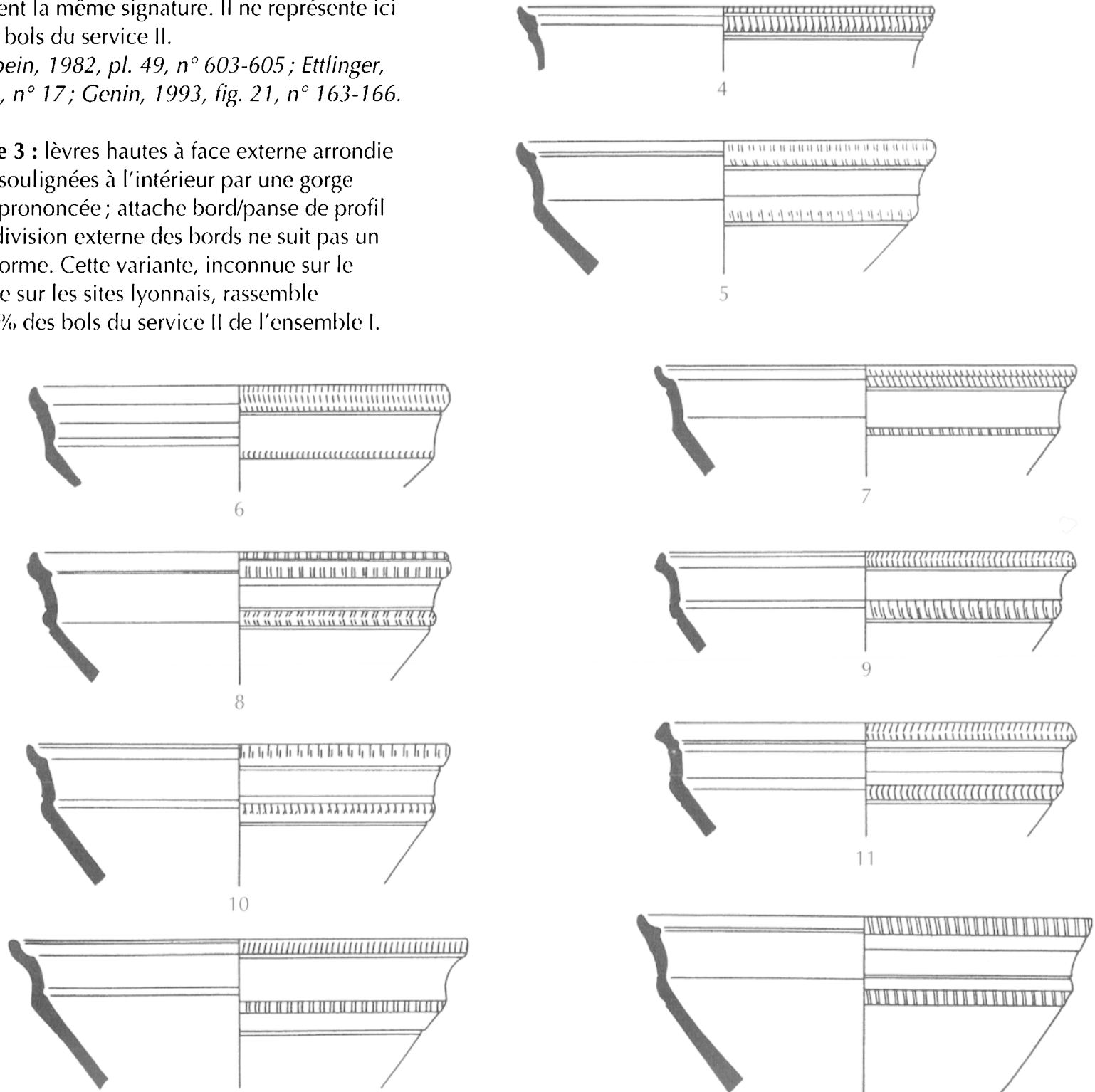

12

Pl. 21. Ensemble I, sigillée, semvice II : bols du grouper 2 (1-3) al du groupe 3 (4-1.3) (échelle : 1/2). 


\section{PI. 22. ENSEMBLE I}

\section{Bols du service II, groupes 4 (194 ex.) et 5 (12 ex.)}

$1-8$, groupe 4 : bols de grands diamètres à bords droits ou légèrement incurvés et à division externe tripartite; les lèvres sont régulièrement soulignées à l'intérieur par un fin sillon et forment un bourrelet à leur extrémité supérieure; de rares exemplaires ne présentent pas ce sillon (2-3) et offrent un profil général beaucoup plus évasé.

Ces bols se rapprochent plus sûrement que ceux des groupes précédents de formes attestées à l'époque augustéenne; on relève en effet quelques parallèles aussi bien à Neuss qu'à Haltern mais toujours en nombre relativement restreint. von Schnurbein, 1982, pl. 44, n 950-953; Ettlinger, 1983, pl. $42, n^{\circ} 5$.

9-11, groupe 5 : bords hauts, incurvés, à division externe irrégulière. Ce groupe très faiblement représenté semble préfigurer les formes classiques les plus couramment attestées de bols du service II ; encore doit-on préciser qu'il ne représente ici qu'à peine plus de $1 \%$ des bols du service II du premier ensemble.
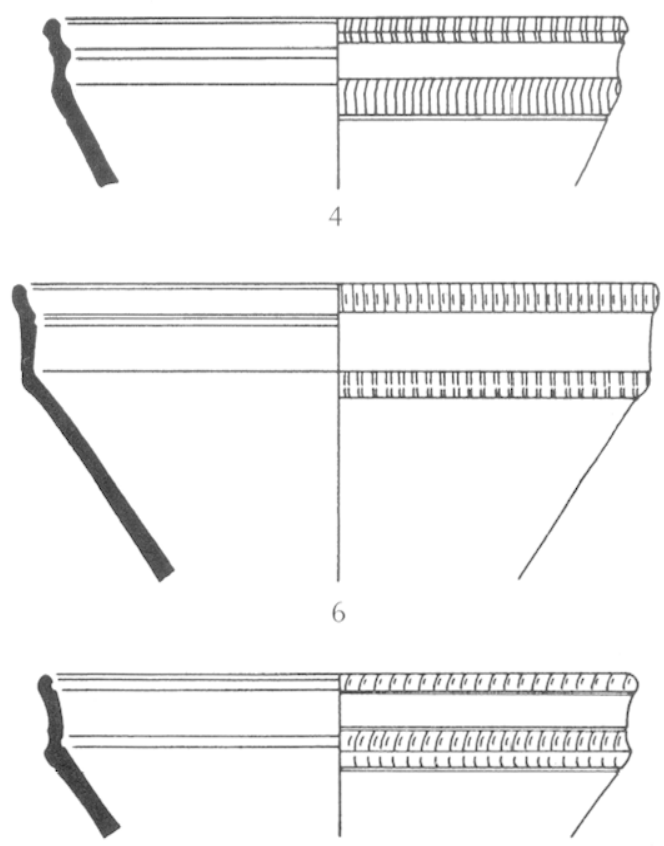

8

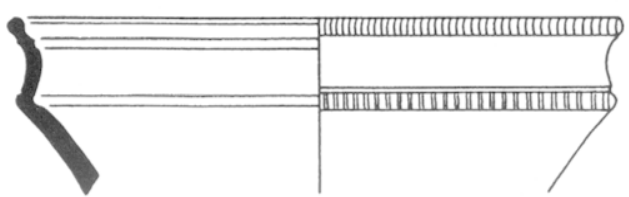

10
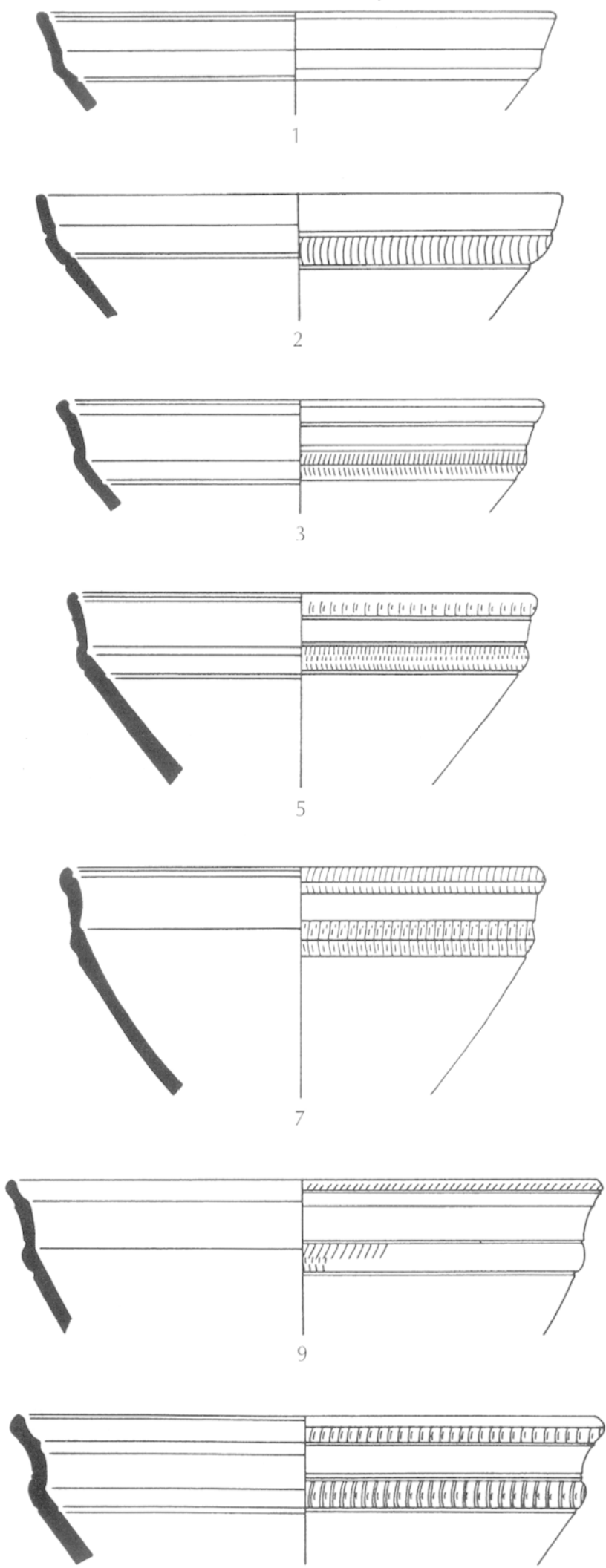

11

P1. 22. Ensemble I, sigillée, semvice II : bols du groupe 4 (1-8) et du groupe 5 (9-11) (échelle : 1/2). 


\section{PI. 23. ENSEMBLE I, DIVERS}

Cent vingt-neuf vases déterminent huit groupes distincts :

1, assiettes à bord oblique (18 ex.) ; 2-3, bols à bord oblique ( 91 ex.); 4, gobelets Haltern 16 ( 2 ex.) ; 5 , bol Haltern 10 (1 ex.); 6-7, bols Haltern 14 (6 ex.) ; 8-9, coupes Haltern 15 (?) (2 ex.); 10, couvercles Haltern 17 (8 ex.); décorée, 1 ex. (fragment à décor végétal, non illustré).

1-3 : les assiettes et les bols à bord oblique constituent l'essentiel du groupe. Les assiettes présentent des vernis érodés de très mauvaise qualité contrairement aux bols qui offrent pour la plupart un aspect fini et des vernis orangé à rouge bien conservés. Les profils des bols évoquent de façon évidente les formes classées à Haltern dans le type $10 \mathrm{~A}$ : les bords sont hauts et fins, tous de très grands diamètres (180-210 mm environ) et très évasés.

von Schnurbein, 1982, pl. 63, n० 1528-1529, pl. 64 et pl. 65, n' 1536-1540; Ettlinger, 1983, pl. 27, n० 1-5, 7, 8; Genin, 1994, fig. 7, n' 91-104.

4 : les gobelets cylindriques $\mathrm{Ha} .16$ produits en céramique à parois fines par l'atelier de Loyasse avant d'être inscrits au répertoire de la Muette, apparaissent très rarement en sigillée.

von Schnurbein, 1982, pl. 70, $n^{c}$ 1642-1643; Genin, 1994, fig. 8, n० 129-130.

5 : le type Ha. 10 est représenté par un fragment surcuit de bord arrondi non divisé semblable aux bords de quelques exemplaires attestés à Haltern. Il s'agit là aussi d'un type assez rare, initialement défini comme " service III ".

von Schnurbein, 1982, pl. 63, n० 152.3, 1525.

6-7 : les bols $\mathrm{Ha} .14$ font partie de ces types produits en marge sous Auguste. Les recherches récentes menées à Cavaillon et à Lyon sur des ensembles de céramiques augustéennes précoces permettent aujourd'hui de rattacher ces bols au répertoire préclassique de la production sigillée.

Schindler, Scheffenegger, 1977, pl. 1, n' 7a; von Schnurbein, 1982, pl. 68, n 1609 ; Ettlinger, 1983, pl. 52, n० 19; Desbat et alii, 1989, fig. 68, n०22 et fig. 76, n०2; Doray, 1992, fig. 2, n० $1-2$ et fig. 3, n० 3-6 Genin, 1994, fig. 7, n० 115-128.

8.9 : deux coupes à bord court, nettement concave et à parois légèrement obliques posent un problème d'identification; elles n'appartiennent pas à un type bien défini et pourraient éventuellement évoquer les coupes de type Ha. 15, produites elles aussi de façon marginale sous Auguste.

von Schnurbein, 1982, pl. 69, n' 1613 (type 15) et pl. $70, n^{\circ} 1648$ (forme unique); Ettlinger, 1983, pl. 50 $n^{\circ} 30$ (gobelets divers).

10 : huit couvercles fins à bords guillochés sont assimilables au type $\mathrm{Ha}$. 17. Il s'agit d'un type encore plus rare que les précédents pour lequel on ne trouve pas d'autres références que celles de Haltern. von Schnurbein, 1982, pl. 70, n 1644-1645.
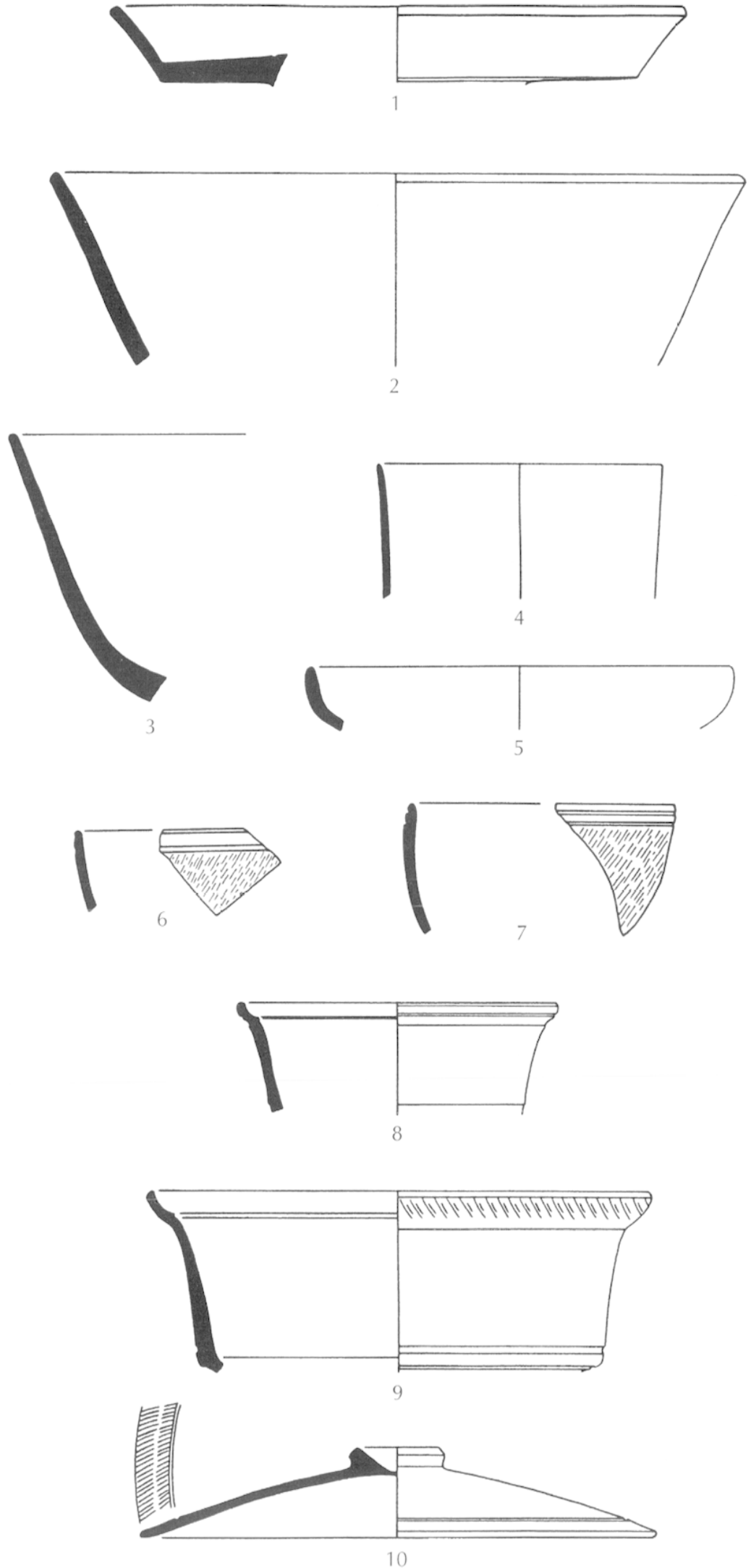

Pl. 23. Einsemble I, sigilliop: divers (échelle: 1/2). 


\section{PI. 24. ENSEMBLE II}

\section{SERVICE IB : plats (168 ex.), assiettes (887 ex.), bols} (1868 ex.)

1-6: les plats de l'ensemble II sont identiques à ceux qu'on a pu observer dans le mobilier de l'ensemble I, si l'on excepte quelques exemplaires isolés à bord interne strié évoquant des formes peut-être intermédiaires entre le service IB et IC (3). Les lèvres sont droites, rainurées à leur extrémité supérieure et la division bipartite interne soulignée par un renflement très épais.

Goudineau, 1968, type 15 ; Ettlinger, 1983, pl. 3, $n^{\circ}$ 15-26; Schindler, Scheffenegger, 1977, pl. 22, $n^{\circ} 1-2$; Genin, 1993, fig. 16, $n^{\circ} 1$.

7-9: les assiettes se répartissent inégalement entre les groupes 1 et 2 précédemment définis. Le groupe 2 (" assiettes type Lyon ») représentent désormais l'essentiel du lot avec 840 ex., soit $95 \%$.

von Schnurbein, 1982, pl. 2, n' 14 et pl. 3, n०36-38; Genin, 1993, fig. 19, $n^{\circ} 57$.

\section{0-16 : bols du service IB, groupe 4 (1387 ex.)} et groupe 5 (477 ex.)

Des trois groupes précoces de l'ensemble l, ne subsiste plus que le groupe 2 ; encore faut-il préciser qu'il se réduit ici à quatre exemplaires (non illustrés). La quasi-totalité du lot est en fait constituée par les groupes $4(\mathbf{1 0 - 1 5})$ et $5(\mathbf{1 6})$ : bols type Lyon $(74 \%)$ et bols proches type Lyon à large rainure interne $(25,5 \%)$. Parmi ces derniers un bol porte la signature de STAB (ILIO); ce timbre est le seul témoin de cette marque sur l'ensemble du mobilier des deux périodes de production.

"Bols type Lyon ": von Schnurbein, 1982, pl. 4-5 et pl. 6, n० 77-80; Ettlinger, 1983, pl. 32, n० 1-11;

Genin, 1993, fig. 20, $n^{\circ} 85-87$.
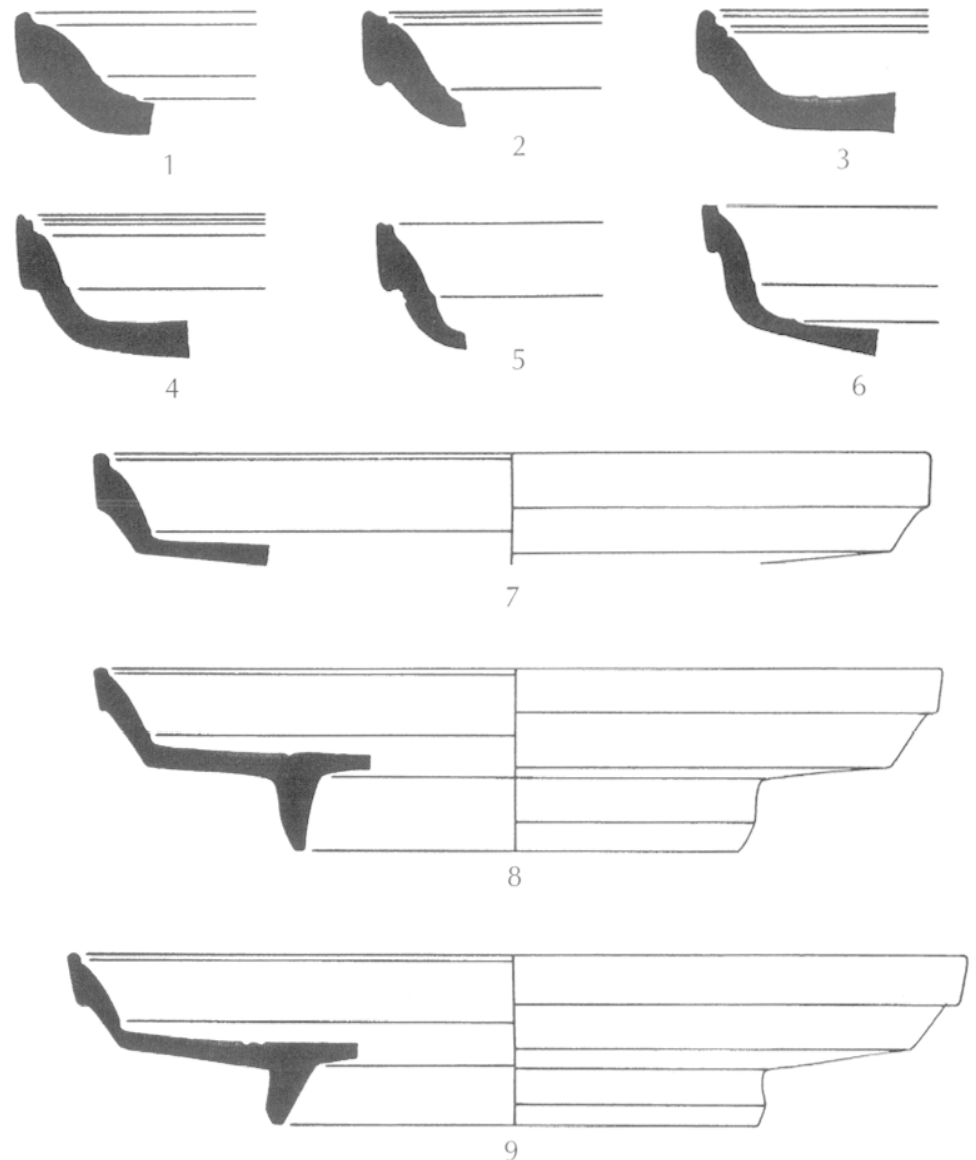

(ixi

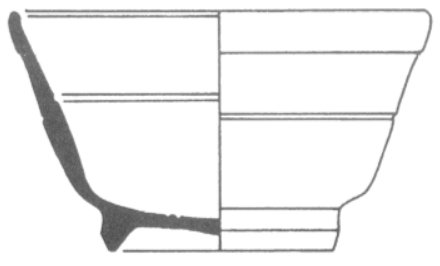

10

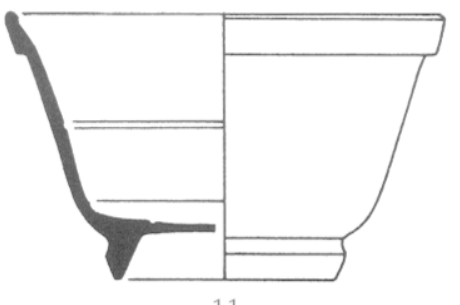

11

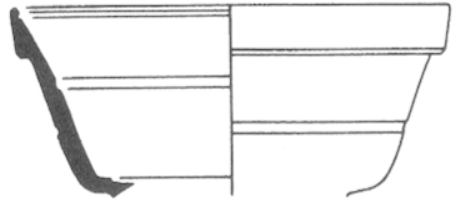

13

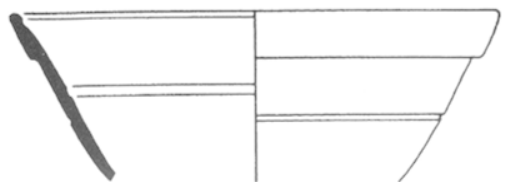

14

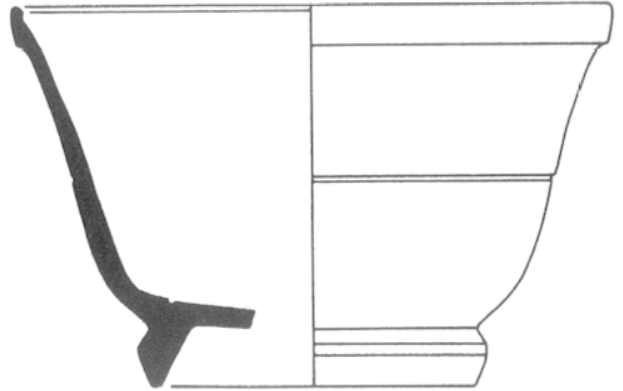

15

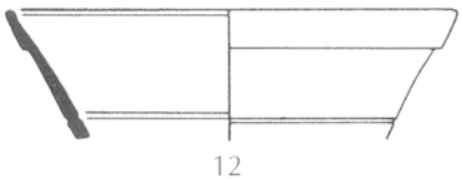

[5AB

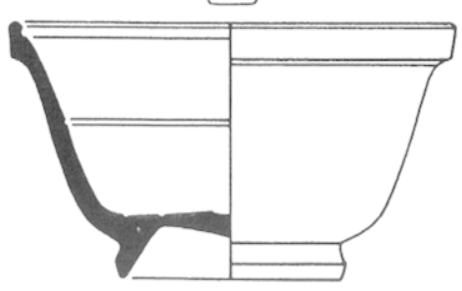

16

P1. 24. Einsemble II, sigillée, service IB : plats (1-6), assiettes (7-9), bols du groupe 4 (10-15) et du groupe 5 (16) (échelle: 1/2). 


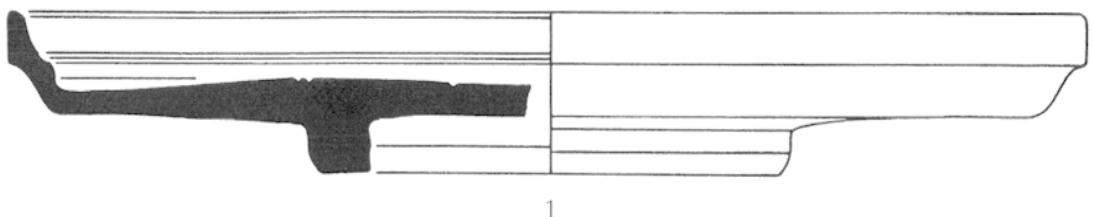

\section{PI. 25. ENSEMBLE II}

\section{SERVICE IC, plats (607 ex.) et assiettes (4 508 ex.)}

1-8, plats du service IC : Quelques exemplaires composent un nouveau groupe 3 (1). Le groupe 1 a disparu et le groupe 2 reste largement majoritaire : les lèvres sont épaisses, dégagées par une gorge large et restent collées à la paroi ; I'attache bord/fond est arrondie dans la grande majorité des cas, anguleuse sur quelques exemplaires. Seul le plat $\mathbf{8}$ se distingue des modèles habituels par un bord parfaitement oblique se raccordant au fond par une partie apparemment bombée à l'intérieur et droite à l'extérieur; ce type $d^{\prime}$ attache, bien que peu fréquent, se rencontre parfois sur des plats du service II (Ha. 3/Goud. 28).

Goudineau, 1968, type 17, variantes b et $c$; von Schnurbein, 1982, pl. 7-9-11; Ettlinger, 1983, pl. 6, $n^{\circ} 1-4$; Schindler, Scheffenegger, 1977, pl. 22, n 5-6.

9-18, assiettes du service IC : les groupes 1 à 4 définis pour le premier ensemble ne sont pratiquement plus représentés dans le second si l'on excepte quelques bords isolés. Le groupe 5, groupe des formes classiques, offre ici un faciès homogène qui pourrait se décalquer sur celui des assiettes IC de I'horizon Haltern et des principaux contextes classiques de référence. On notera qu'une partie de ces assiettes sont des produits des potiers RASIN (IVS) et L. T (H) YRS (VS).

Goudineau, 1968, type 17, variantes b et $c$; von Schnurbein, 1982, pl. 16, n०159-163 et pl. 17, n० 171 174 ; Ettlinger, 1983, pl. 15-16; Genin, 1993, fig. 19, $n^{\circ} 66-81$.
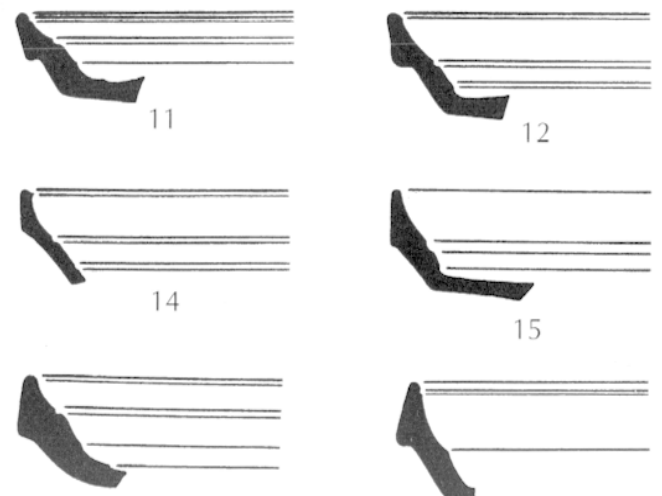

16
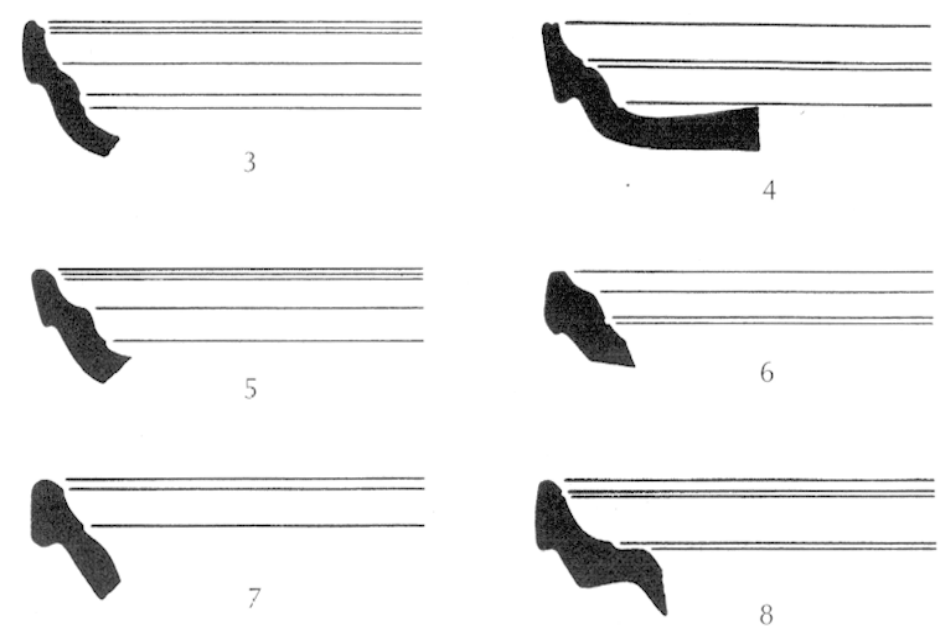

RASIN
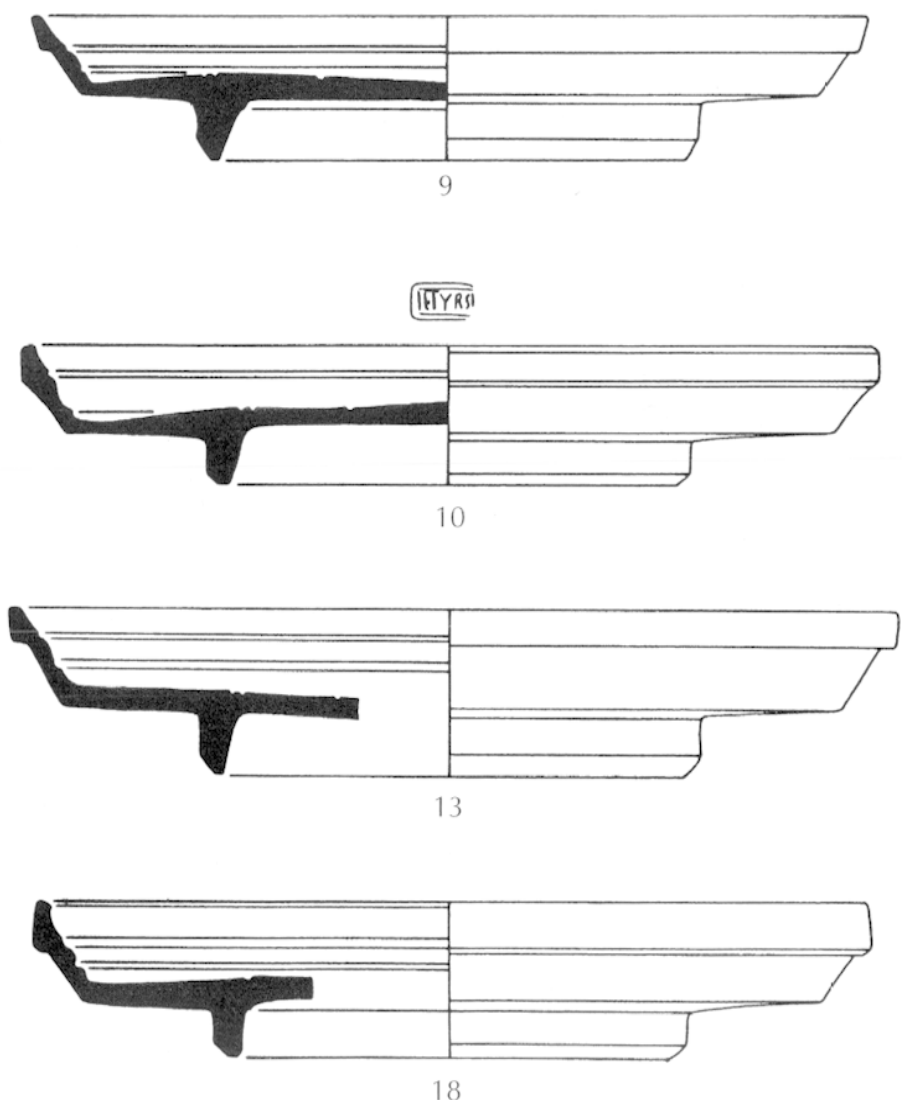

Pl. 25. Ensemble II, sigillée, service IC: : plats du groupe 3 (1) et du groupe 2 (2-8), et assiettes du groupe 5 (9-18) (échelle: 1/2). 


\section{PI. 26. ENSEMBLE II}

\section{BOLS DU SERVICE IC (400 ex.)}

Ils sont très inégalement distribués entre les quatre groupes initialement définis.

- Le groupe 1 (bords arrondis de petits diamètres à face interne concave) se réduit à un seul exemplaire (non illustré).

- Le groupe 2 (bords courts de section triangulaire nettement détachés du col) ne compte plus que 10 ex., soit 2,5\% du $\operatorname{lot}(\mathbf{1})$.

- Le groupe 3 (lèvres hautes et fines collées à la paroi) connaît une augmentation notable avec 53 ex. qui représentent plus de $13 \%$ des bols (2-4).

von Schnurbein, 1982, pl. 23, $n^{\circ} 394-396 b$ et pl. 24, $n^{\circ} 396 \mathrm{C}$; Ettlinger, 1983, pl. 37, n०11; Genin, 1993, fig. 20, $n^{\circ} 95$.

- Le groupe 4 (profils classiques), qui formait déjà 73,5\% du lot précédent, culmine ici à $84 \%$ avec 336 ex. (5-10). On n'observe aucune différence typologique entre l'échantillon de l'ensemble I et celui de l'ensemble II; il s'agit de formes pour lesquelles on trouve un très grand nombre de références à l'époque classique.

von Schnurbein, 1982, pl. 23, n 382-389 c; Ettlinger, 1983, pl. 35-36-37; Genin, 1993, fig. 20, n० 91-93.
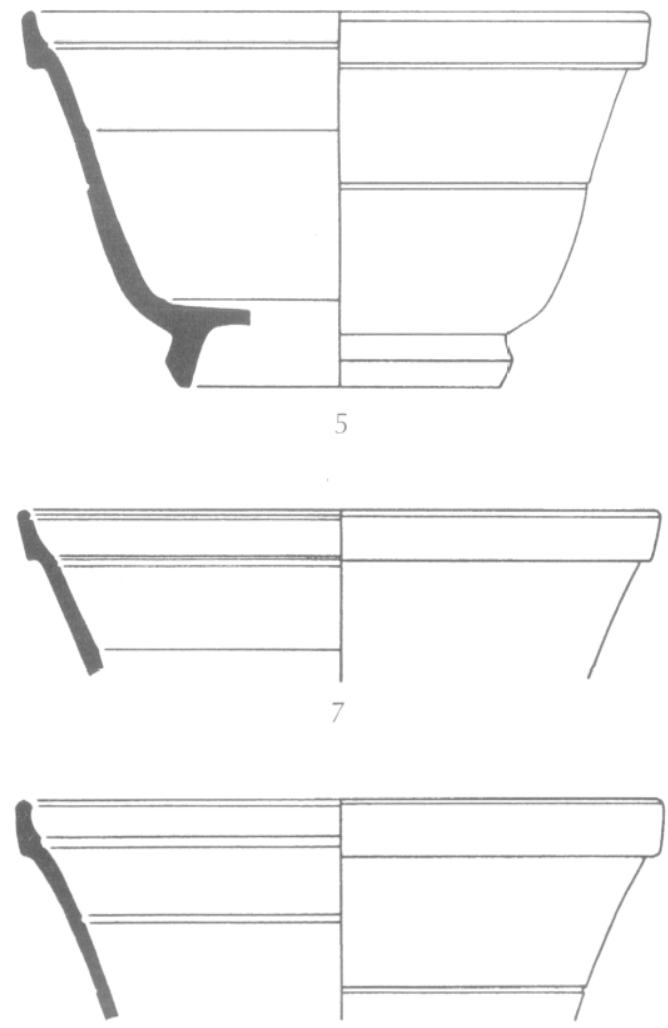

P1. 26. Ensemble II, sigillée, senvice IC: : bols du groupe 2 (l), du groupe 3 (2-4) et du groupe 4 (5-I0) (échelle : I/2).
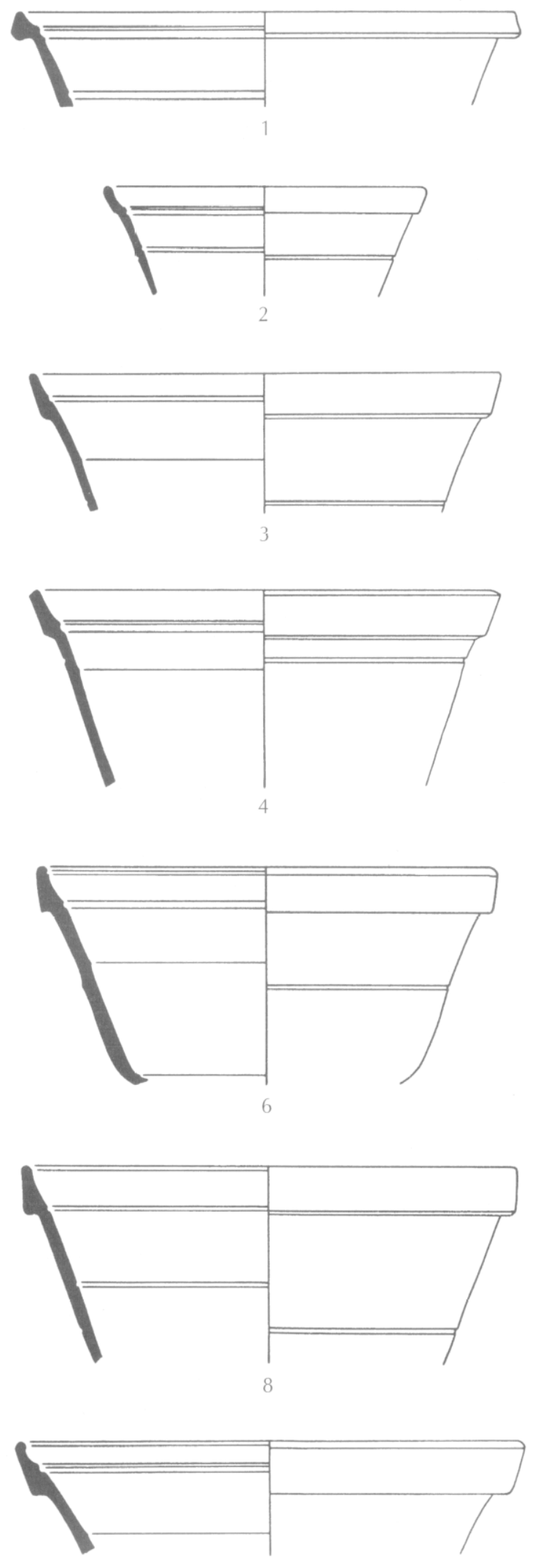

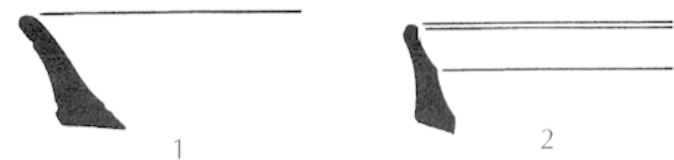

\section{PI. 27. ENSEMBLE II}

\section{SERVICE II, plats (127 ex.) et assiettes (292 ex.)}

1-11, plats du service II : ils ne figuraient pas dans le mobilier de l'ensemble I. Les exemplaires de l'ensemble II offrent un faciès classique, similaire à celui qu'on rencontre sur les sites de référence : bords droits ou incurvés à triple division externe et division interne bien marquée (6-10). Quelques rebords sont lisses (1-3) ou faiblement divisés (4-5) et un plat se particularise par un double ressaut à la liaison bord/fond peu habituel sur les formes du service II (11) et caractéristique du type Haltern 3.

5 : von Schnurbein, 1982, pl. 31, n570; Genin, 1993, fig. $20, n^{\circ} 98$.

6-10 : von Schnurbein, 1982, pl. 29-31; Ettlinger, 1983, pl. 6, n²0-35 et pl. 7, n० 1-6; Thilhard, 1988, fig. 11, $n^{\circ} 121,124$; Genin, 1993, fig. 20, n०97, 100.

12 : Goudineau, 1968, B-2B-99, 100; von Schnurbein, 1982, pl. 56; Ettlinger, 198.3, pl. 7, n० 8-11; Thilhard, 1988, fig. $15, n^{\circ} 210-212$.

12-20, assiettes du service II : aucune d'entre elles n'appartient au type précoce (bords incurvés non divisés) qui prédominait dans le premier ensemble. II $s^{\prime}$ agit en effet exclusivement de profils classiques se rattachant au type Haltern 2 même si ces assiettes ne sont jamais identiques : les bords sont d'épaisseur variable, plus ou moins droits ou incurvés et les proportions des divisions interne et externe peuvent différer selon les exemplaires.

Goudineau, 1968, type 26; von Schnurbein, 1982, pl. 32-37; Ettlinger, 1983, pl. 19-20 et pl. 21, n०1-22 ; Genin, 1993, fig. 20, n 101-111.

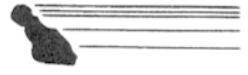

13
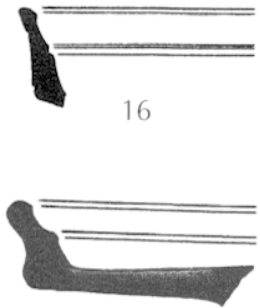

18

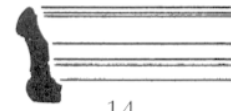

14

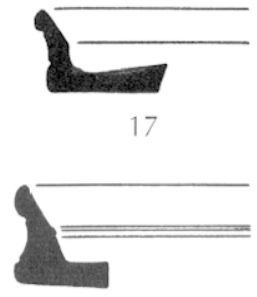

19
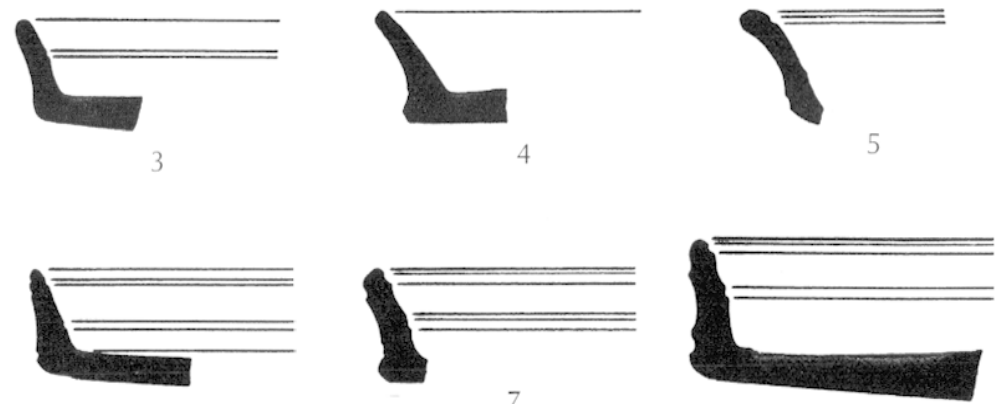

8

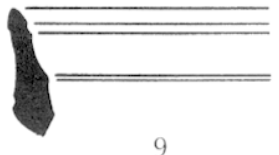

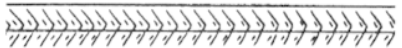

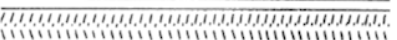

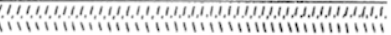

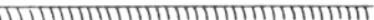

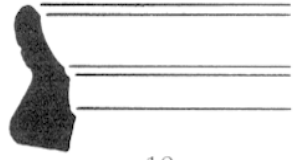

10

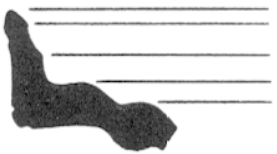

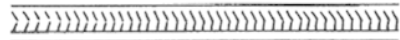

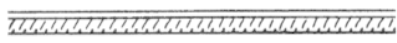

11
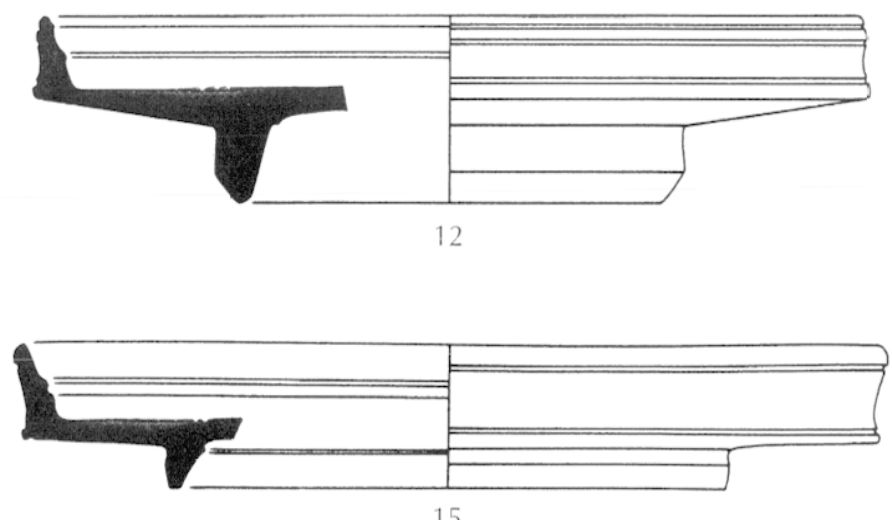

15

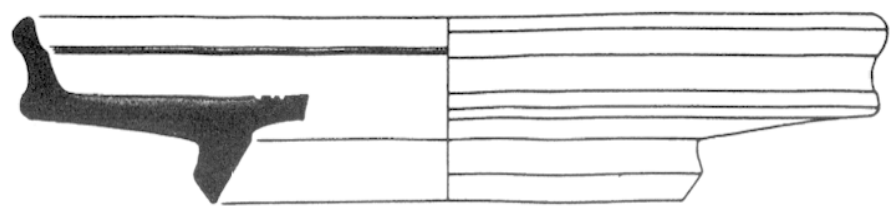

20

Pl. 27. Ensemble II, sigillée, service II : plats (1-11) et assietles (12-20) (échelle : 1/2). 


\section{PI. 28. ENSEMBLE II}

\section{BOLS DU SERVICE II (1 236 ex.)}

Les groupes 1 et 3 qui concernaient deux types de profils précoces pratiquement inconnus sur les sites de référence ne sont plus du tout représentés dans le second ensemble.

Les 1236 bols du service II montrent une évolution typologique évidente vers les formes les plus courantes de ce service.

Le groupe 2 (bords courts et droits de petits diamètres et délimités par des bourrelets guillochés) constitue ici près du tiers de l'échantillon. Le groupe 4 (bords en bourrelet hauts et droits de grands diamètres) rassemble la moitié des bols; le groupe 5, groupe des formes classiques quasiment inexistant dans le premier ensemble, est ici beaucoup plus abondant avec 257 ex. soit $21 \%$ du lot.

\section{Bols du service II, groupes 2 (363 ex.) et 4 (616 ex.)}

\section{1-6, groupe 2}

von Schnurbein, 1982, pl. 49, $n^{\circ}$ 603-605; Ettlinger, 1983, pl. 42, n 17; Genin, 1993, fig. 21, n 163-

166.

$7-14$, groupe 4

von Schnurbein, 1982, pl. 44, n 950-953; Ettlinger, 1983, pl. 42, n०5.
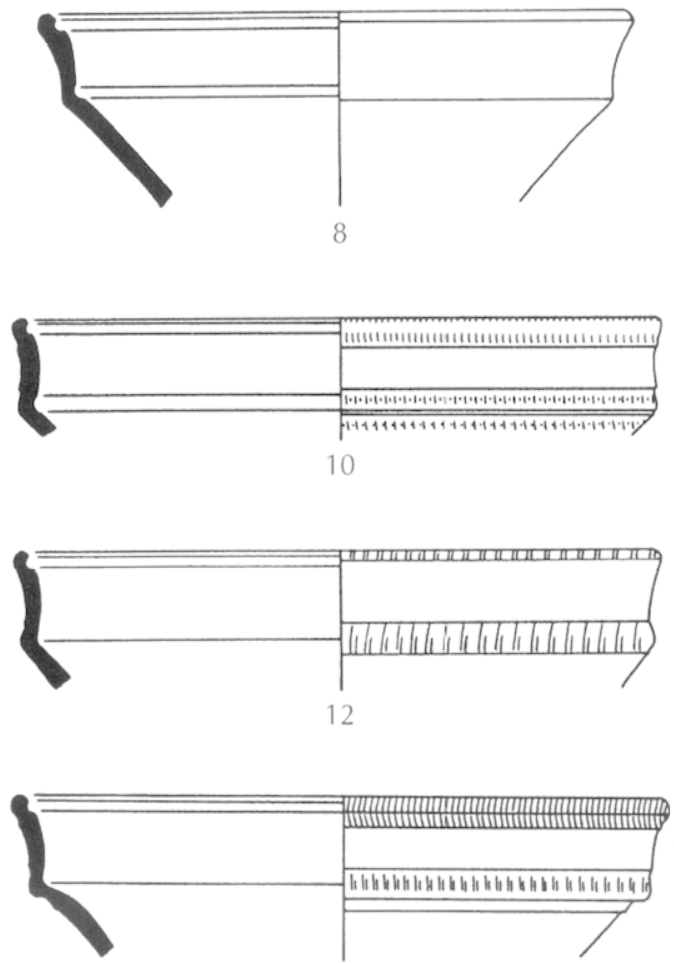

13
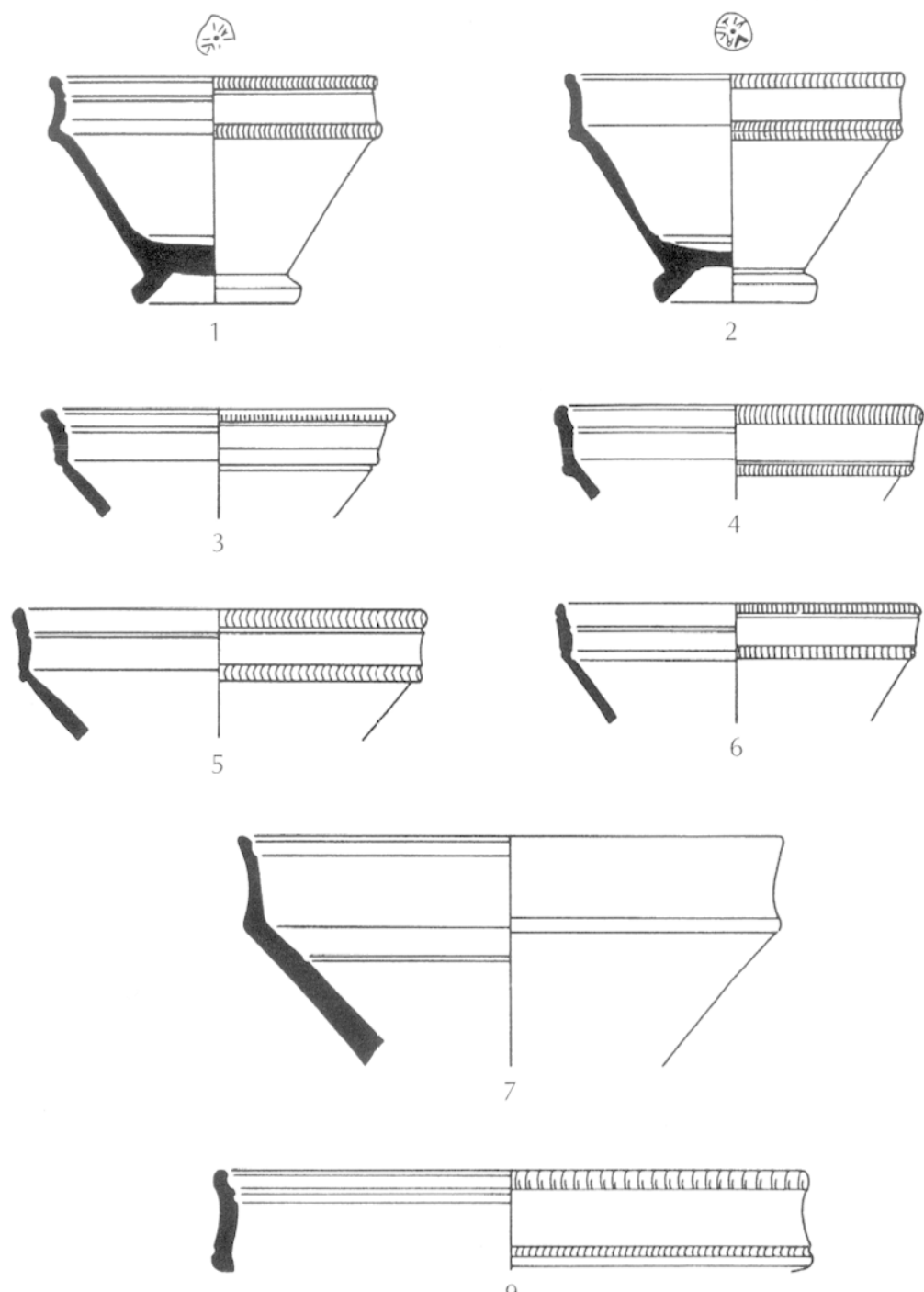

9

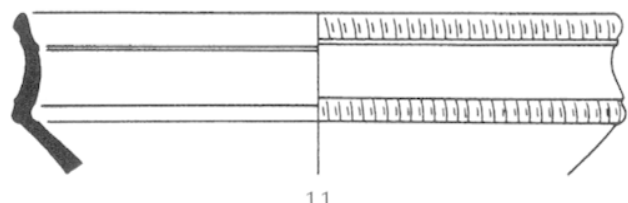

11

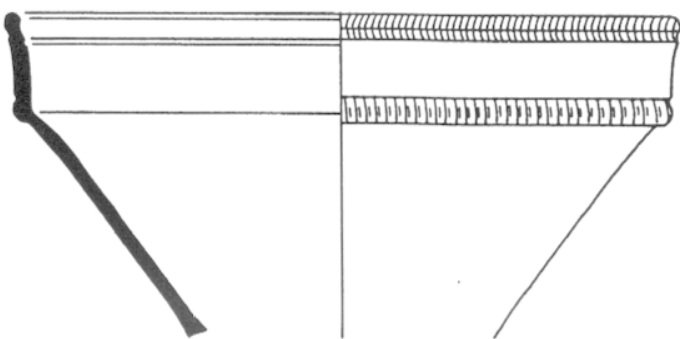

14

Pl. 28. Ensemble II, sigillée, service II : bols du groupe 2 (1-6) et du groupe 4 (7-14) (échelle : 1/2). 


\section{PI. 29. ENSEMBLE II}

\section{Bols du service II, groupe 5 (257 ex.)}

Ce groupe, qui rassemble les formes classiques de bols du service II, passe de $1 \%$ dans I'ensemble I à $21 \%$ dans l'ensemble II. von Schnurbein, 1982, pl. 38-43, pl. 44, n 877 883, pl. 45-51; Ettlinger, 1983, pl. 43-45;

Genin, 1993, fig. 21, n० 112-162.
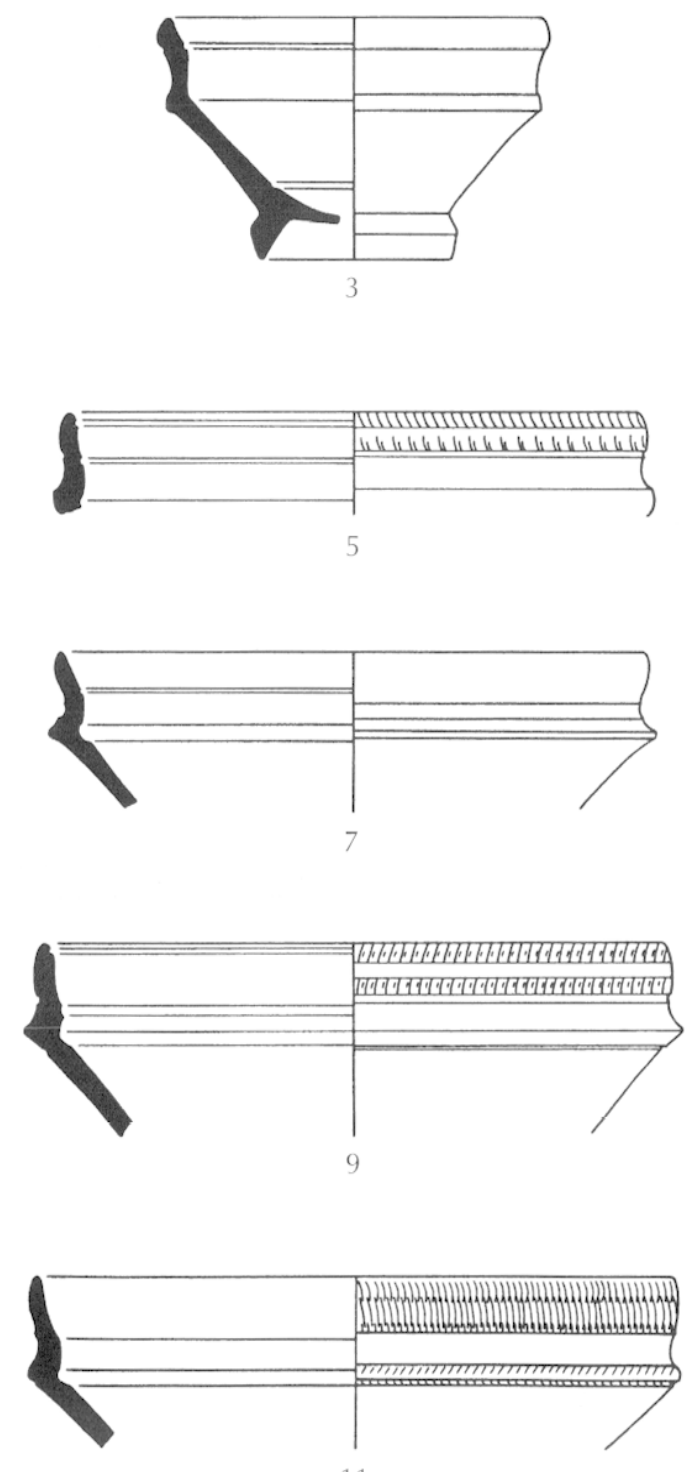
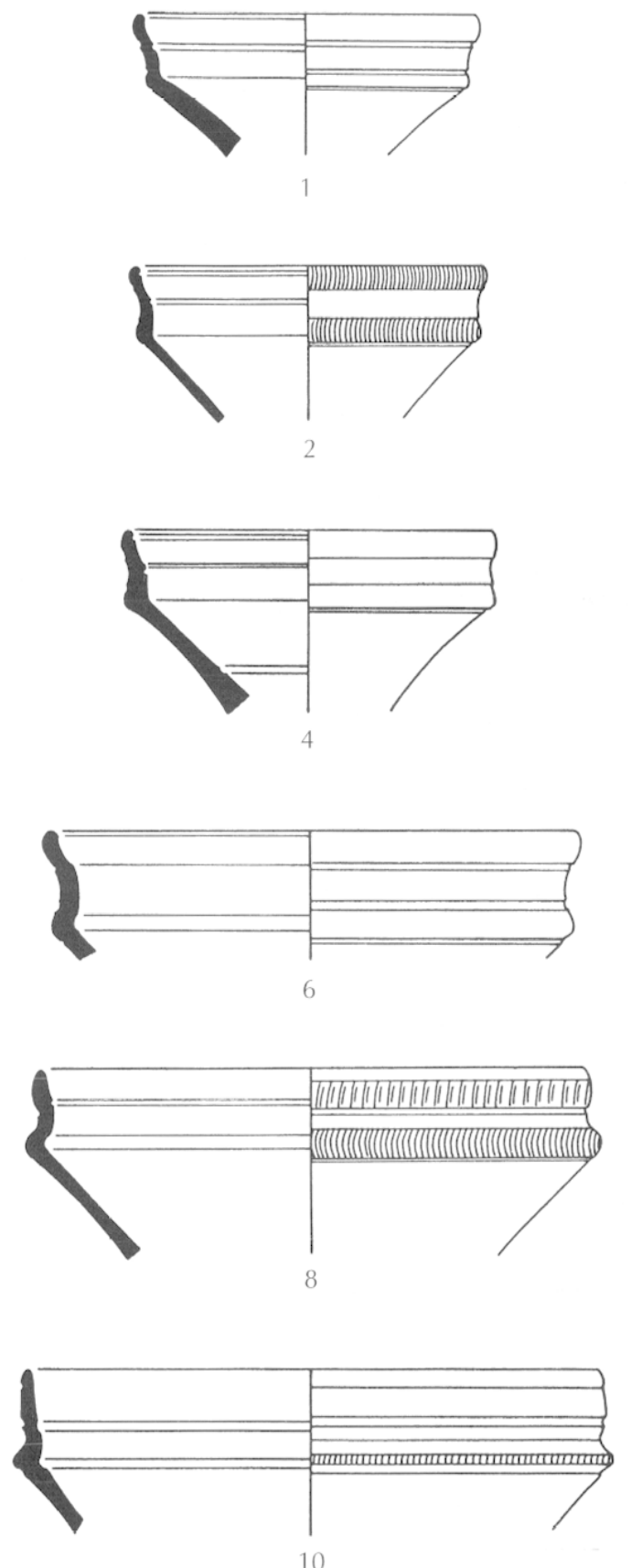

10

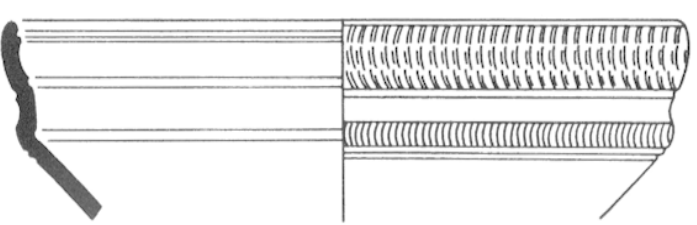

12

P1. 29. Ensemble II, sigillée, service II : bols du groupe 5 (échelle : $1 / 2$ ). 


\section{PI. 30. ENSEMBLE II}

\section{DIVERS (324 ex.)}

Trois des séries représentées dans l'ensemble I ne sont plus attestées : assiettes à bord oblique, tasses Haltern 14, couvercles Haltern 17.

- bols à bord oblique (111 ex.), gobelets Haltern 16 (73 ex.), bols Haltern 10 (103 ex.), coupes Haltern 15 (?) (10 ex.), type lisse indéterminé : (1 ex.), décorée (26 ex.).

La typologie des trois premières séries n'appelle pas de commentaire particulier mais leurs indices de fréquence semblent bel et bien attester des changements notables par rapport à l'ensemble 1 . Les bols à bord oblique, dérivés du type archaïque Goudineau 2, se maintiennent relativement bien avec 111 ex. soit $34 \%$ du lot. Les gobelets Haltern 16 voient leurs effectifs passer de 32 à $23 \%$ des vases.

1-4, bols à bord oblique :

von Schnurbein, 1982, pl. 63, n० 1528-1529, pl. 64-65, n० 1536-1540; Ettlinger, 1983, pl. 27, $n^{\circ}$ 1-5, 7-8; Genin, 1993, fig. 22, n० 168-169. 5-10, gobelets Haltern 16 :

von Schnurbein, 1982, pl. 70, n० 1642-1643; Genin, 1993, fig. 22, n० 170-171.
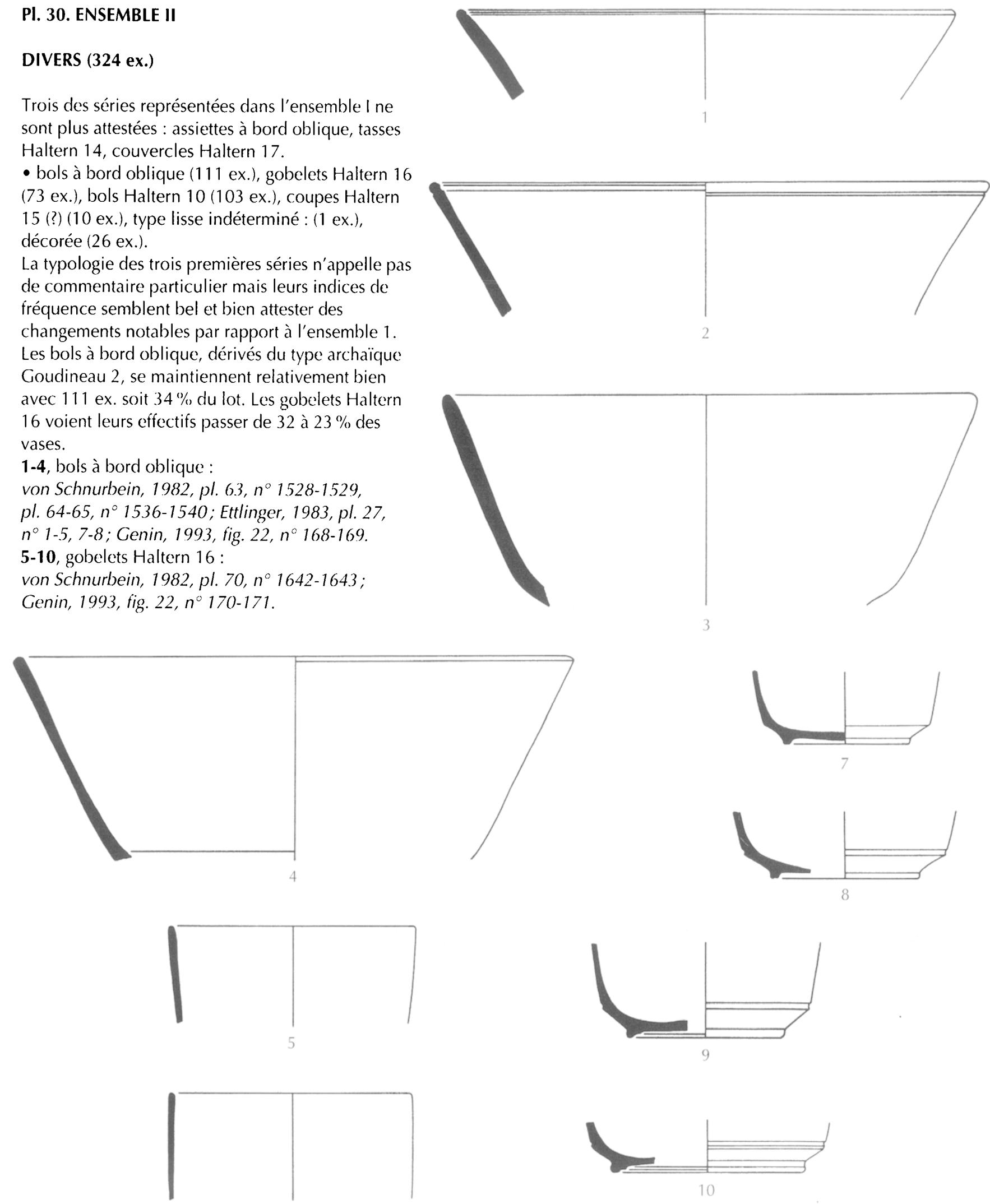

P1. 30. Ensemble II, sigillée : divers (échelle : 1/2). 


\section{PI. 31. ENSEMBLE II}

\section{DIVERS (324 ex.)}

Les bols Haltern 10 sont presque aussi peu représentés qu'auparavant $(1,5 \%$ contre $1 \%$ dans l'ensemble 1). Une dizaine de bords pourrait appartenir à des coupes proches ou dérivées du type Haltern 15. Un seul fragment de petite coupe (?) à collerette n'évoque aucun des types connus sous Auguste.

La sigillée moulée quasiment absente du premier ensemble compte vingt-six fragments de bords, fonds et panses qui déterminent au moins cinq formes identifiables: trois calices de type Drag. 11, un gobelet de L. TETTEI (VS) qui s'apparente au type XIII de Dragendorff et un fond à pied annulaire appartenant peut-être à une tasse ou un gobelet moulé.

1-4, bols Haltern 10 : von Schnurbein, 1982, pl. 63, $n^{\circ} 1523,1525$; Genin, 1993, fig. 22, n० 177.

5.6, coupes Haltern 15 (?) : von Schnurbein, 1982, pl. 69, $n^{\circ} 1613$ (type 15) et pl. 70, $n^{\circ} 1648$ (unique). 7-8, types lisses indéterminés: Ettlinger, 1983, pl. 50, $n^{\circ} 23,29,30$.

9-12, sigillée moulée : Albrecht, 1938, pl. 46, b; Dragendorff, Watzinger, 1948, (types XI et XIII); von Schnurbein, 1982, pl. 86 (type XI); Ettlinger, 1983, pl. 54, n' 1-3 (type XI); Genin, 1993, fig. 22, $n^{\circ}$ 188-189 (type XI).
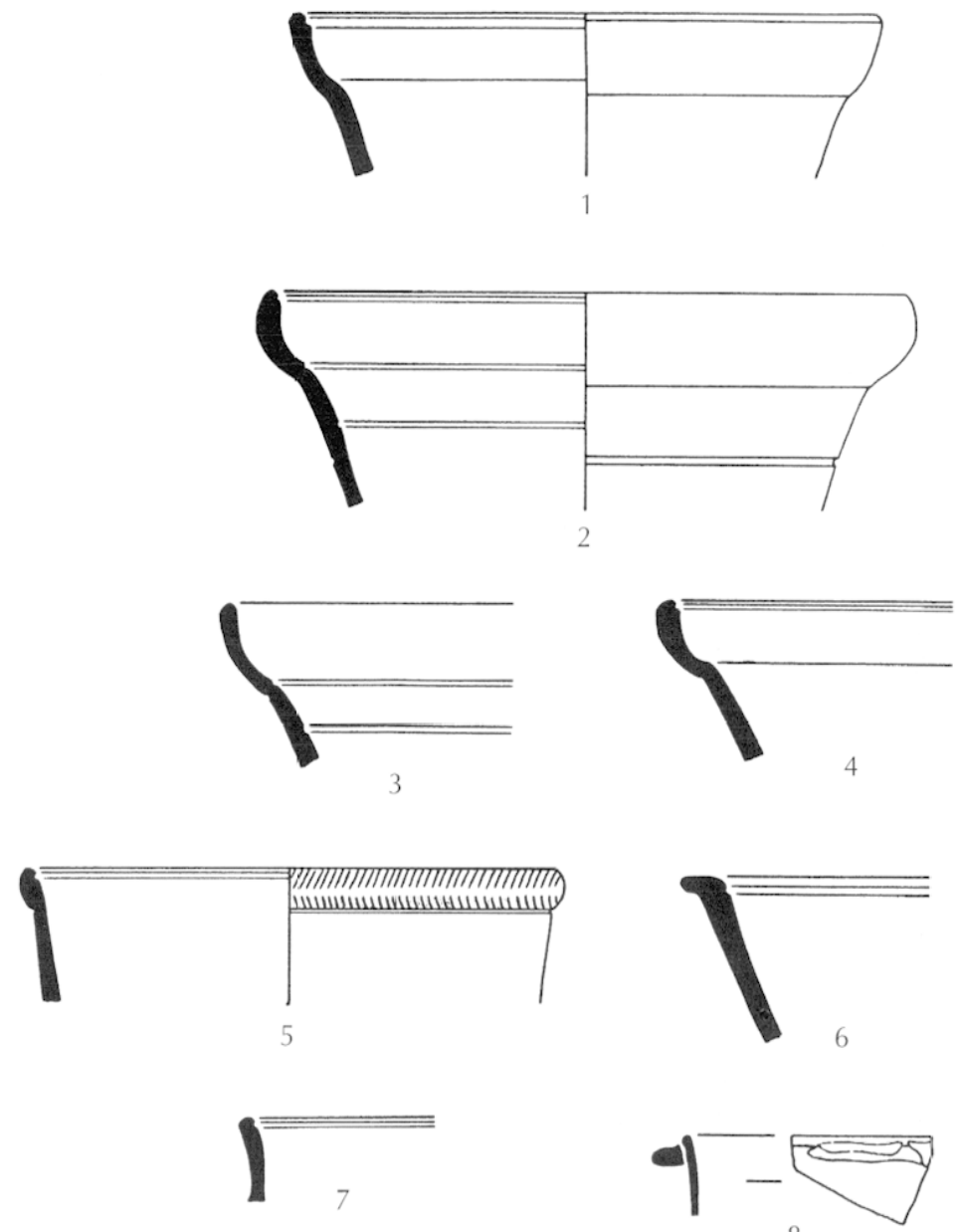
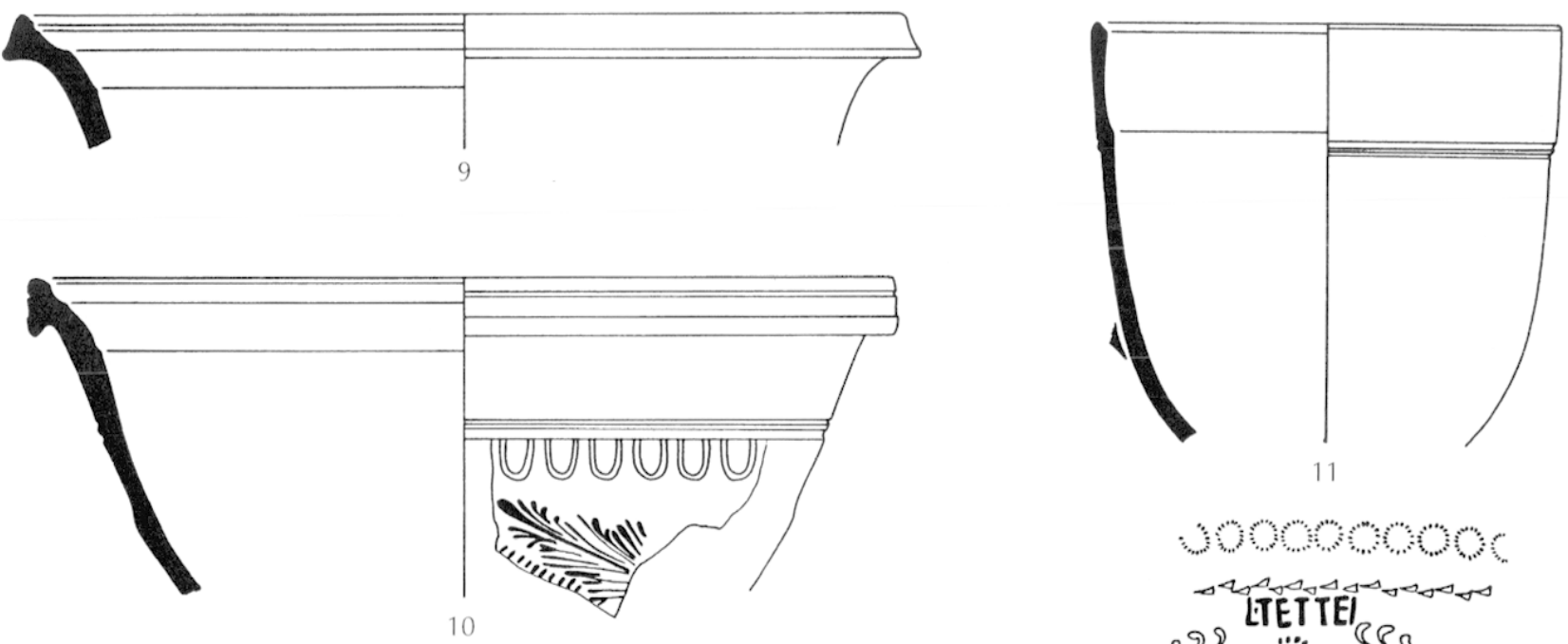

11

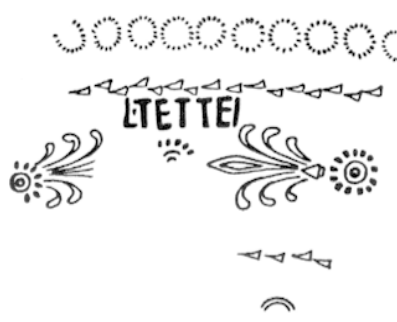

Pl. 31. Ensemble II, sigillée: divers (échelle: 1/2). 

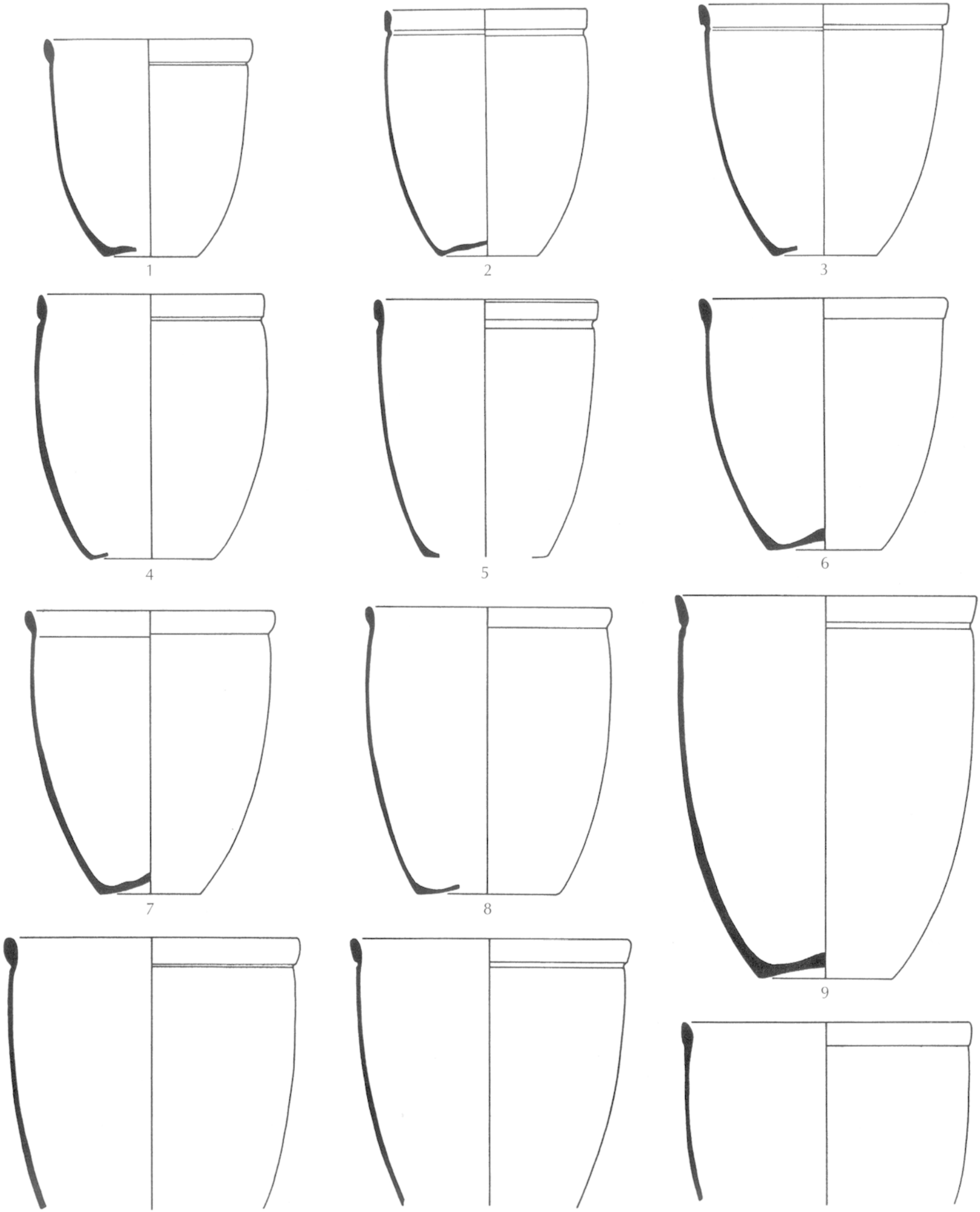

10

11

12

P1. 32. Ensemble I, vases à parois fines : gobelets-tonneaux (échelle : $1 / 2$ ). 

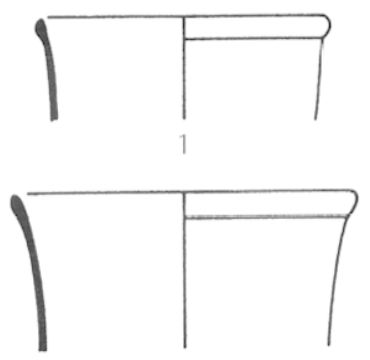

4

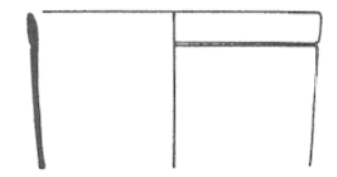

6
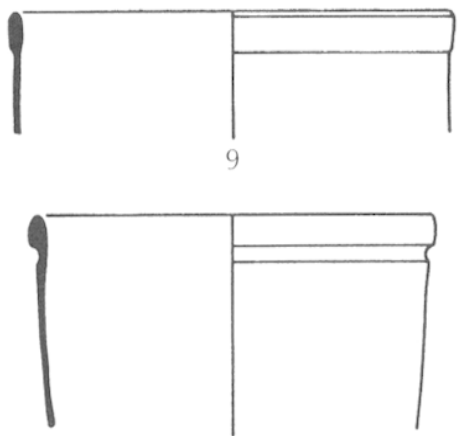

12

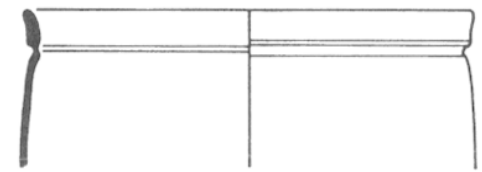

15

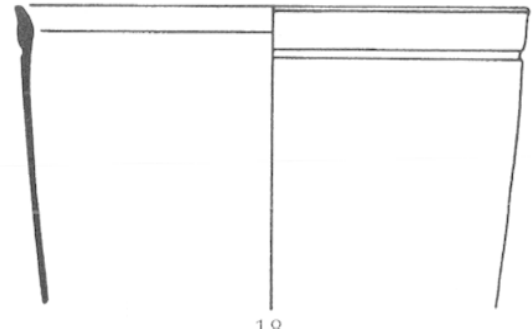

18

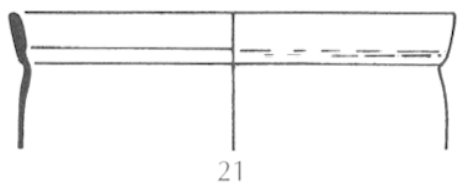

21

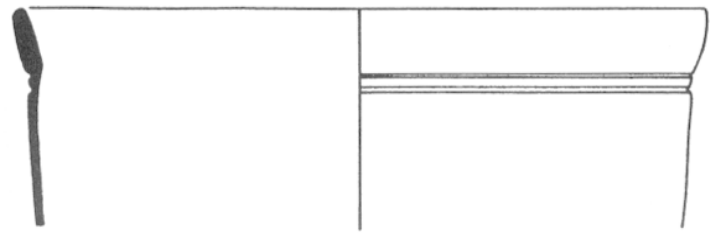

25
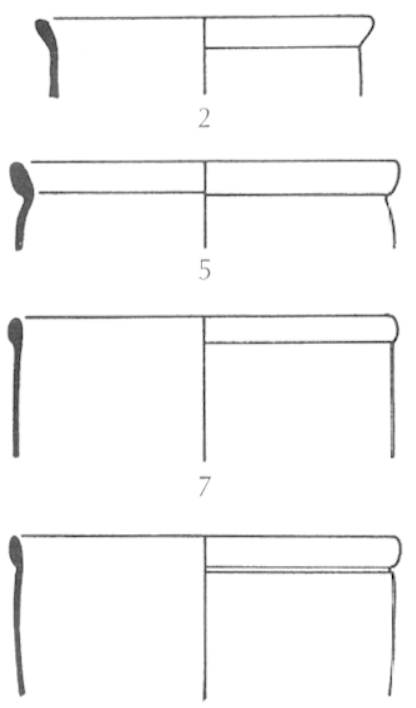

10

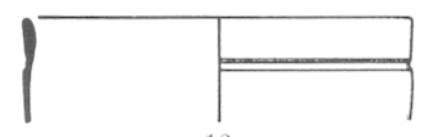

13
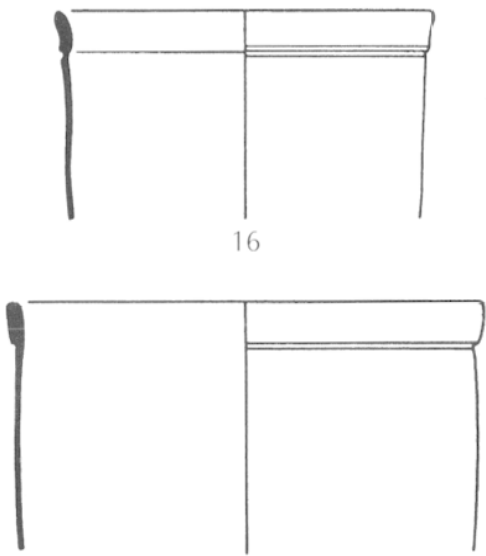

19

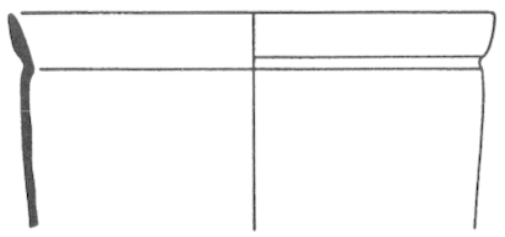

22
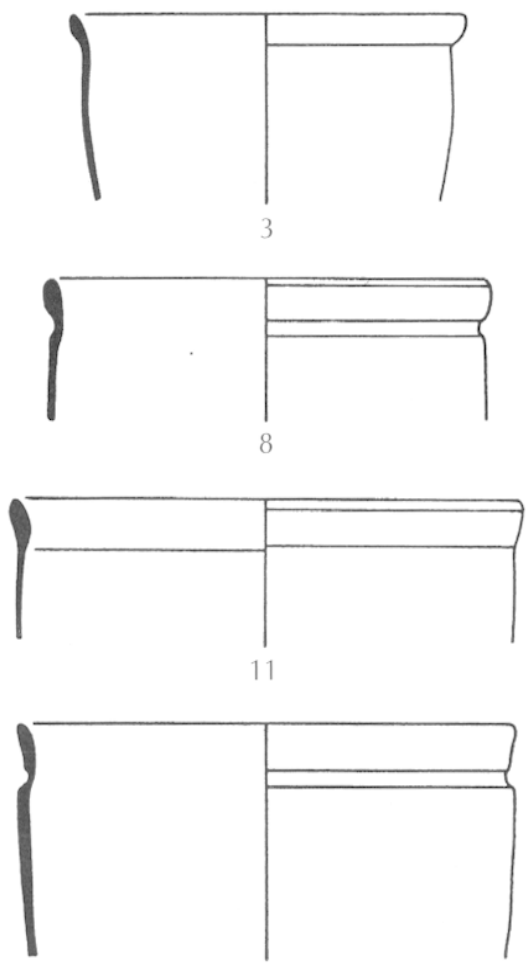

14
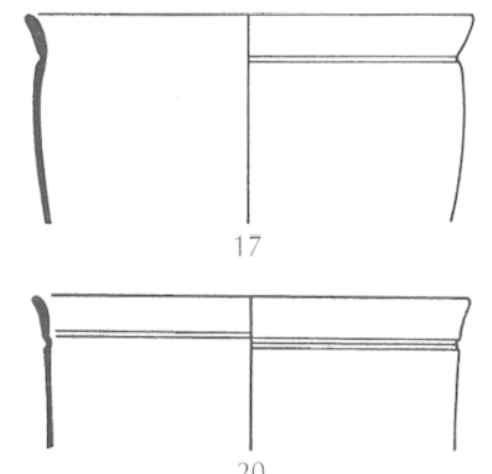

20
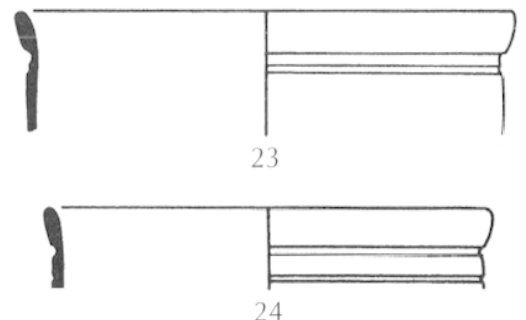

24

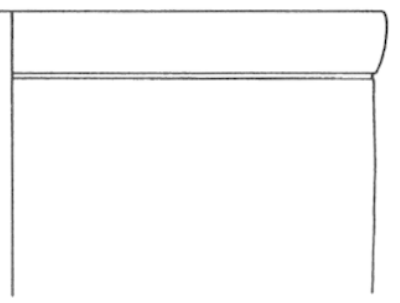

26

P1. 33. Ensemble I, vases à parois fines : gobelets-tomneaux (échelle : 1/2). 

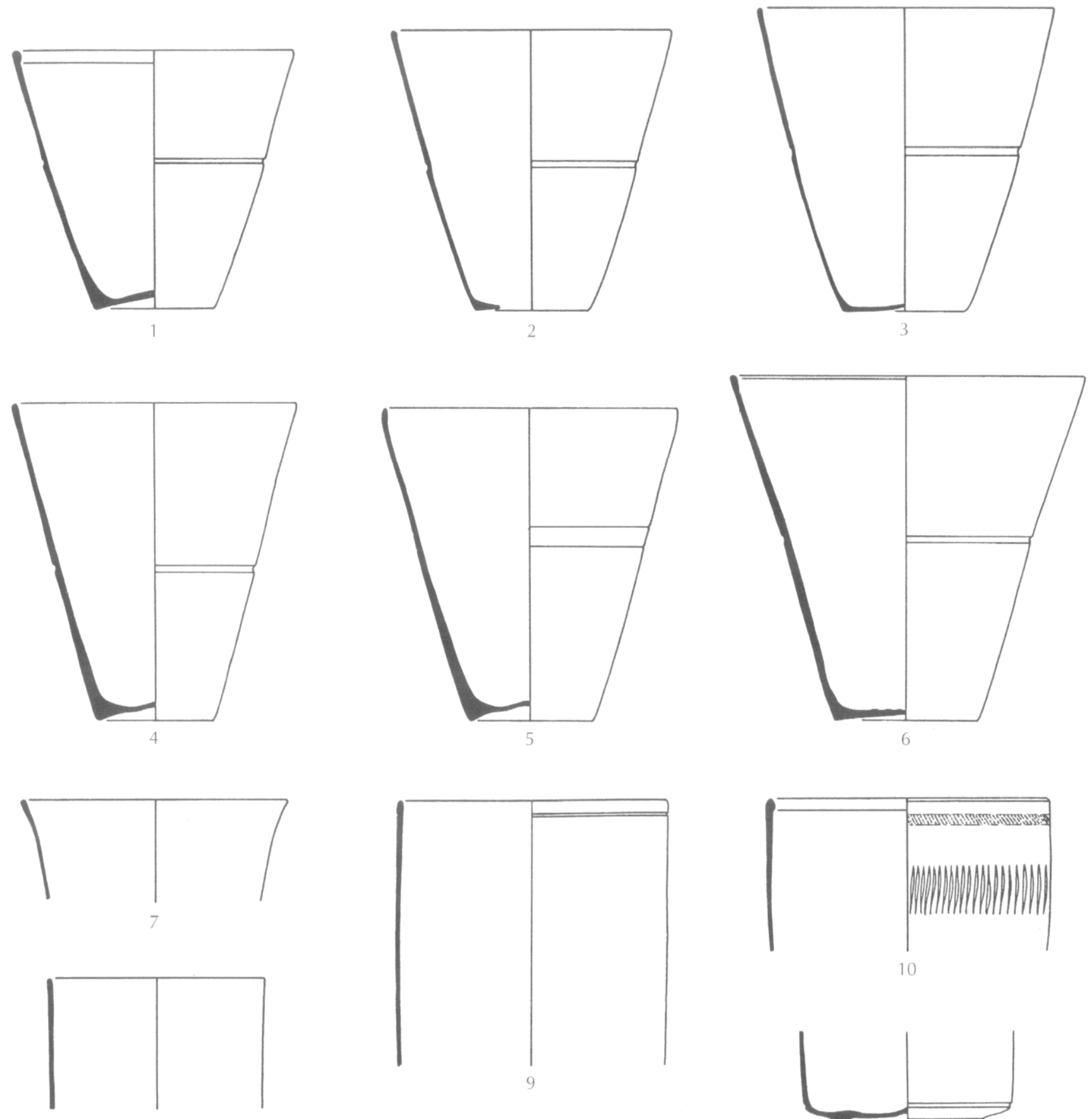

10
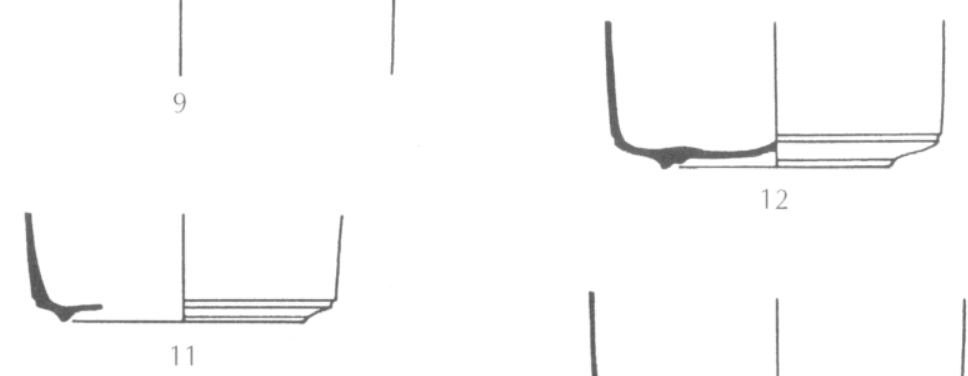

12

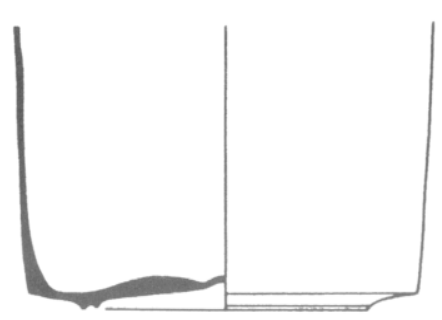

13

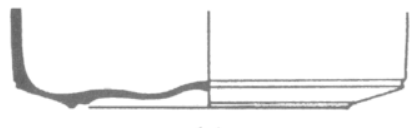

14

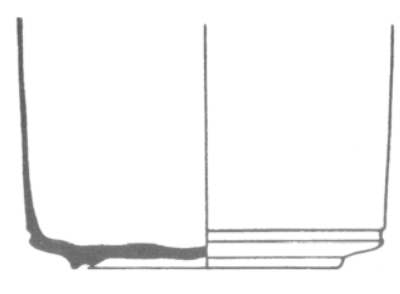

15

P1. 34. Ensemble I, vases à jarois fines : gobelets tronconiques (1-6) el gobelets cylindriques (7-15) (échelle : 1/2). 

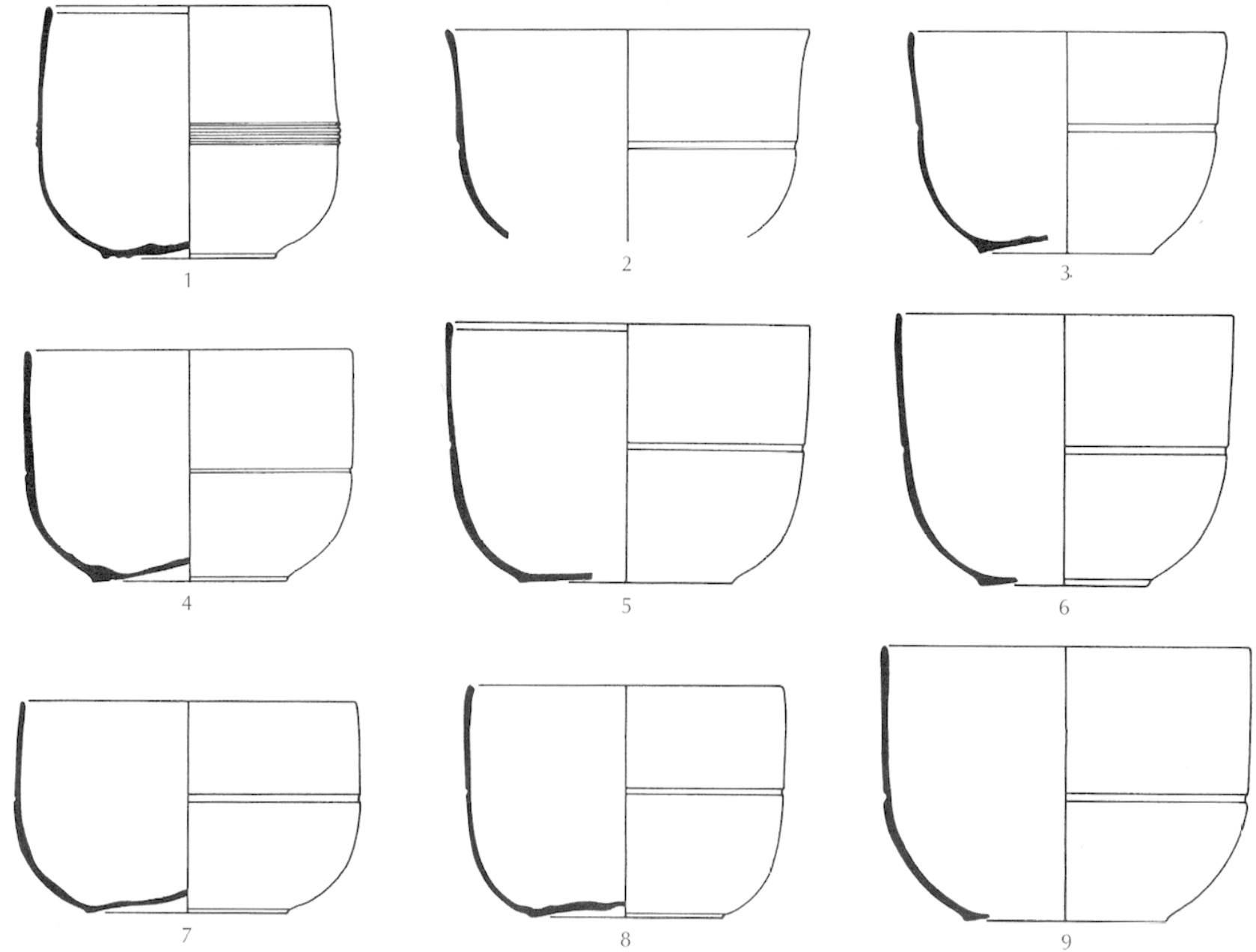
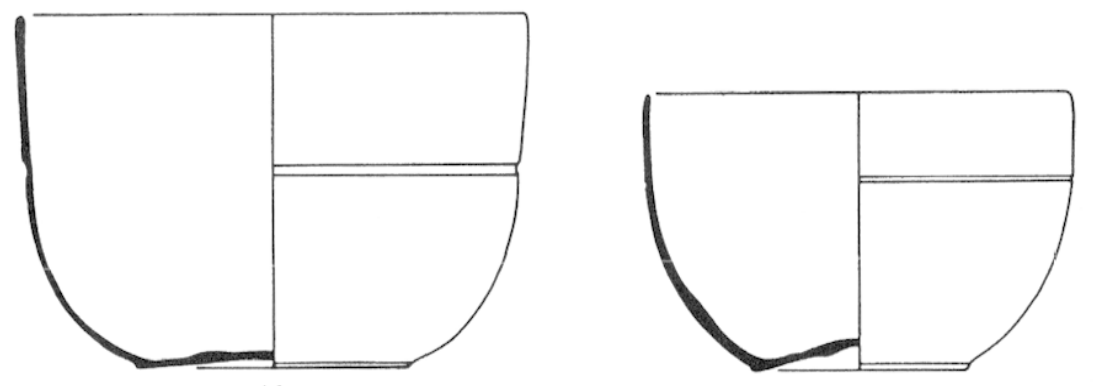

11

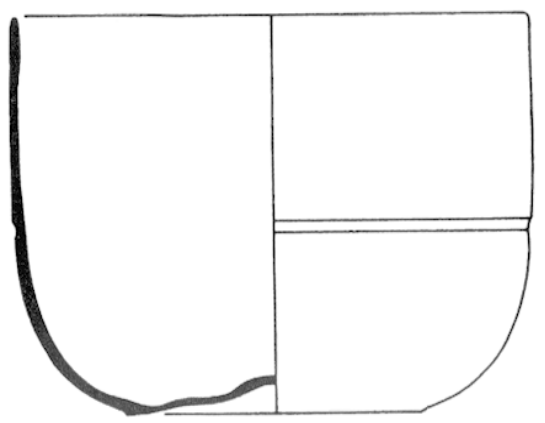

13

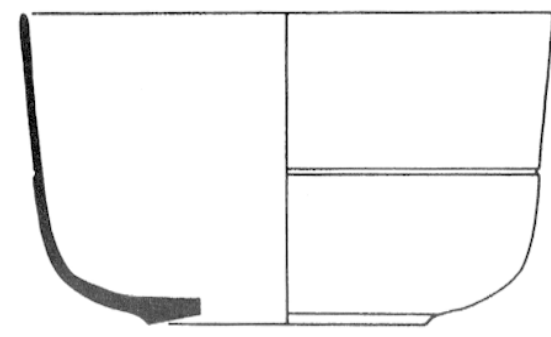

14

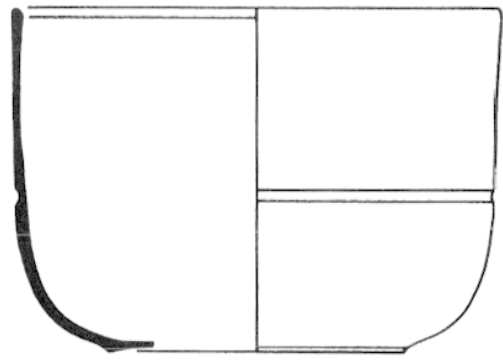

12

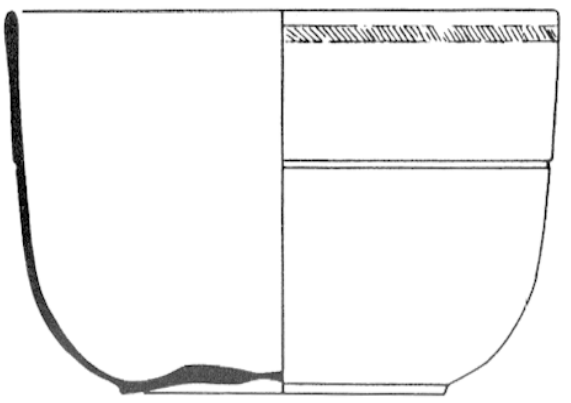

15

Pl. 35. Ensemble I, vases à parois fines : bols hémisphériques (échelle : 1/2). 

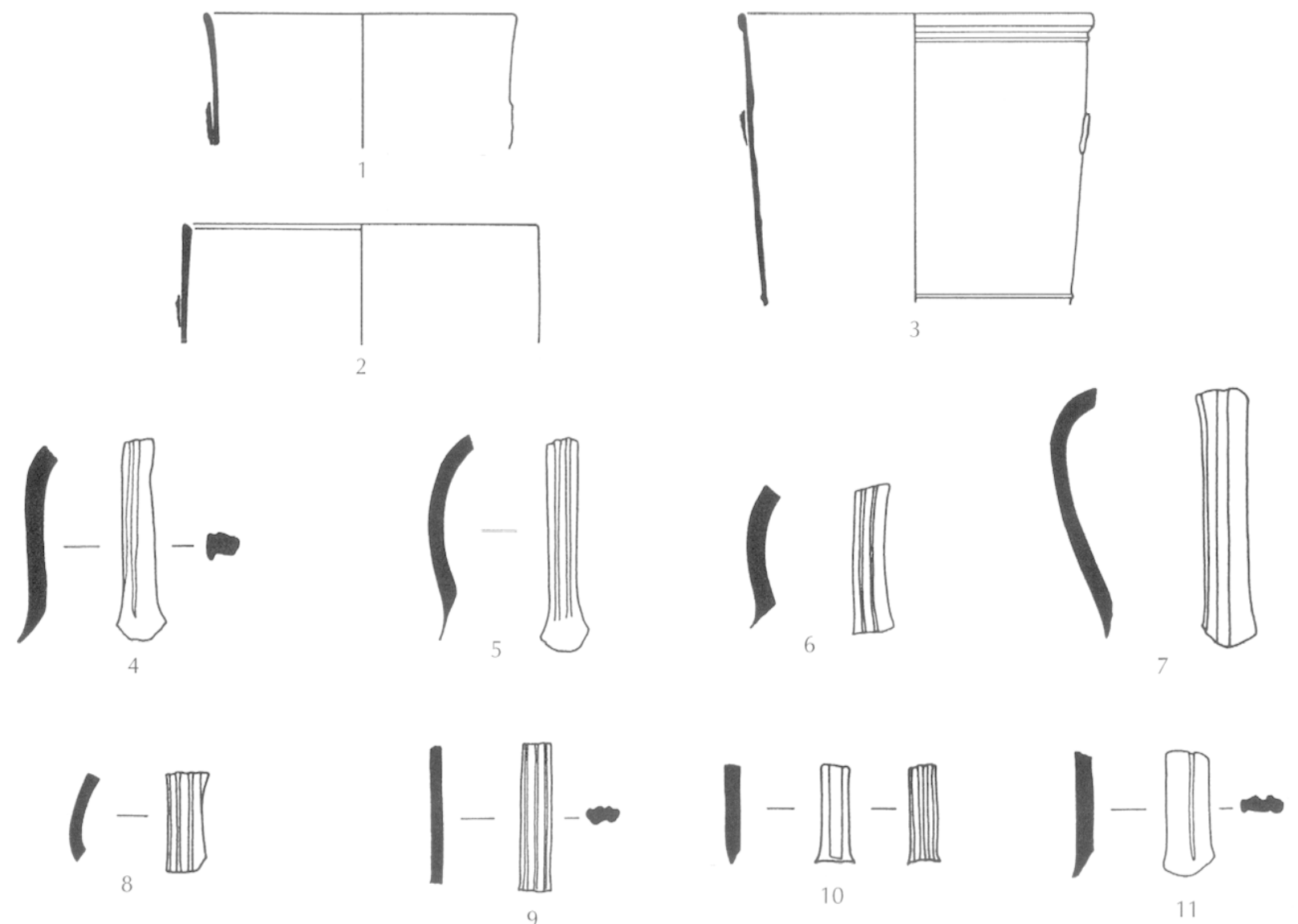

11
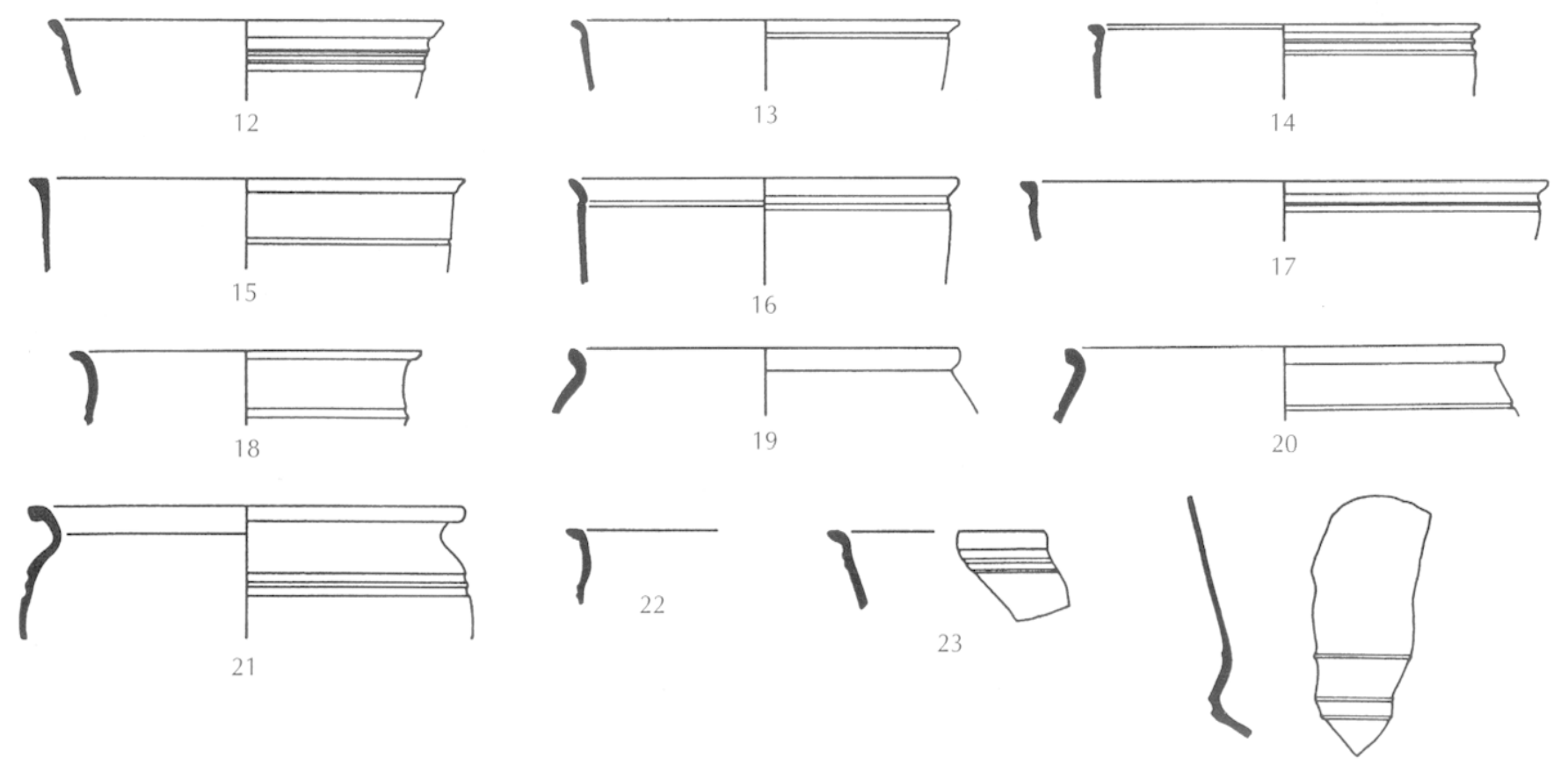

Pl. 36. Ensemble $I$, vases à parois fines à pâte siliceuse : divers (échelle: 1/2). 

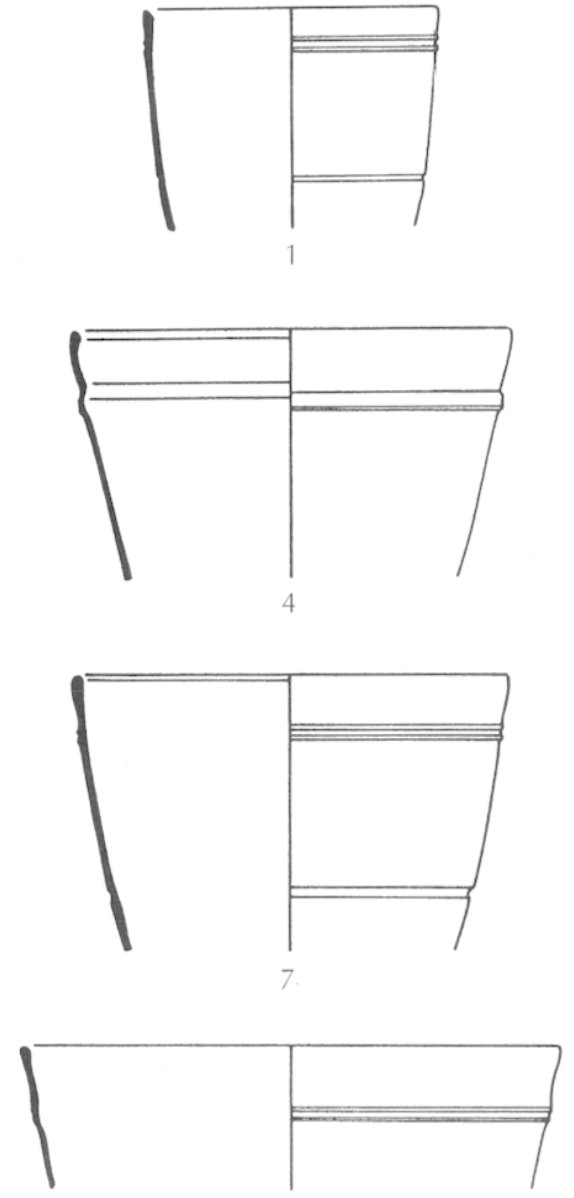

10

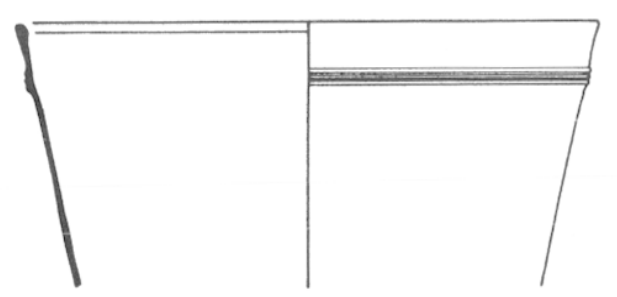

13

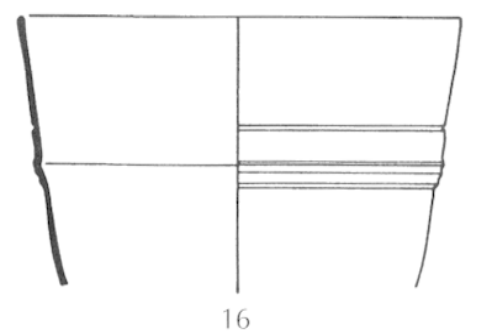

16
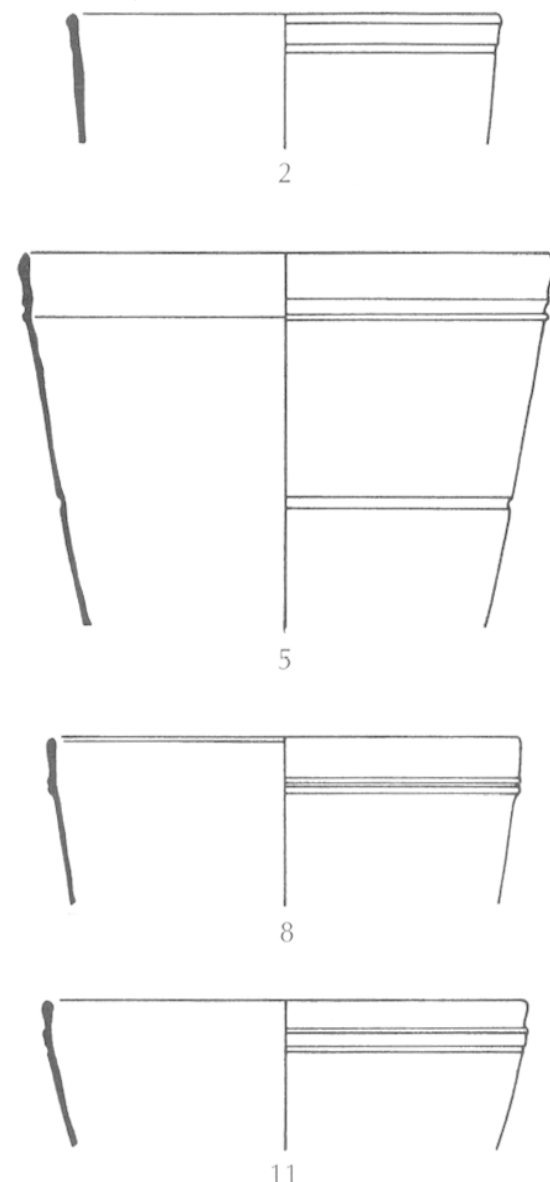

11

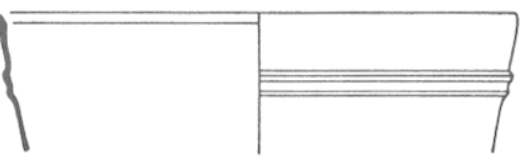

14

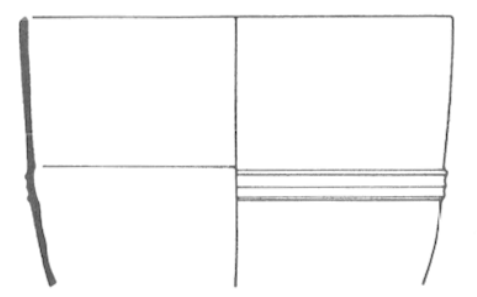

17

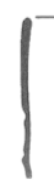

8
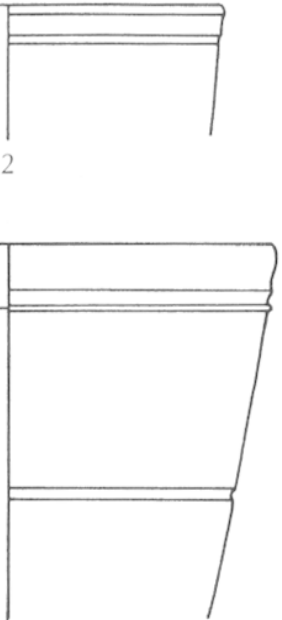

5

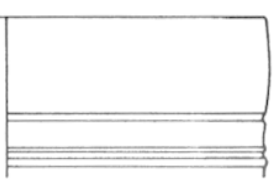

19
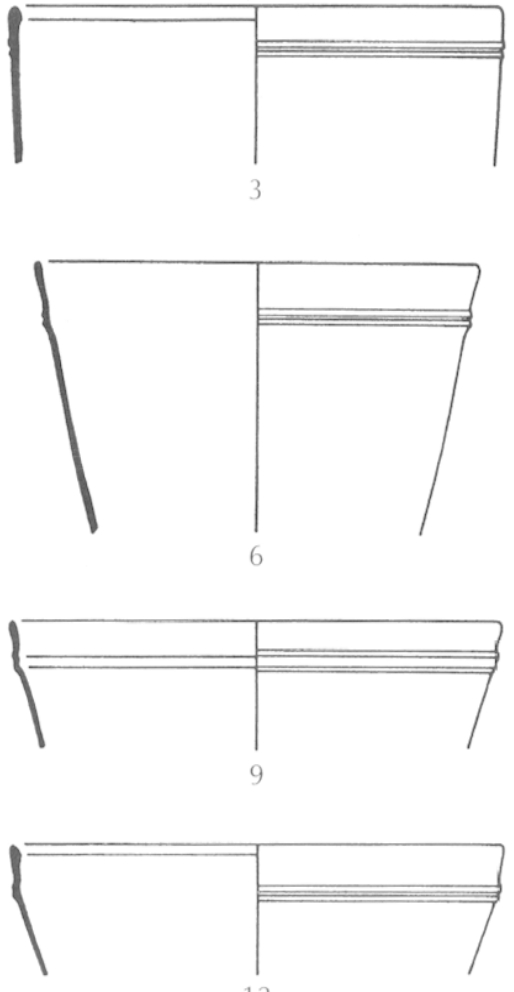

12

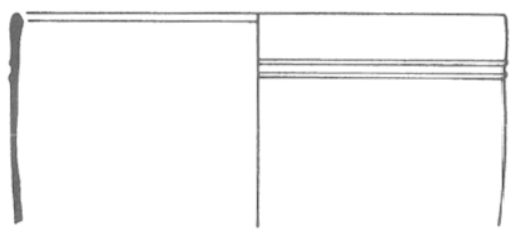

15
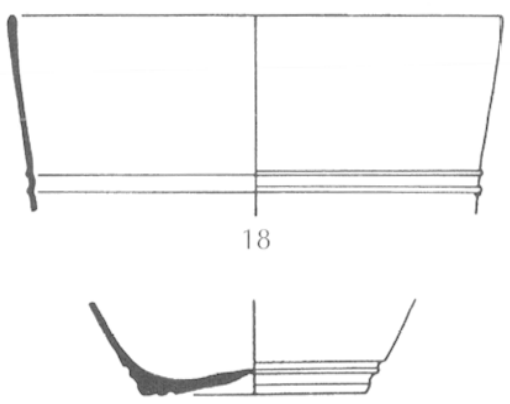

20

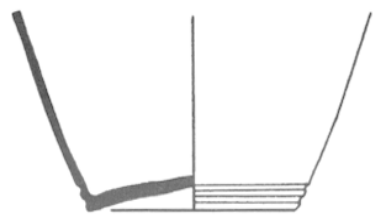

21

P1. 37. Ensemble I, vases à parois fines à pâte siliceuse : gobelets à bords striés (échelle : 1/2). 

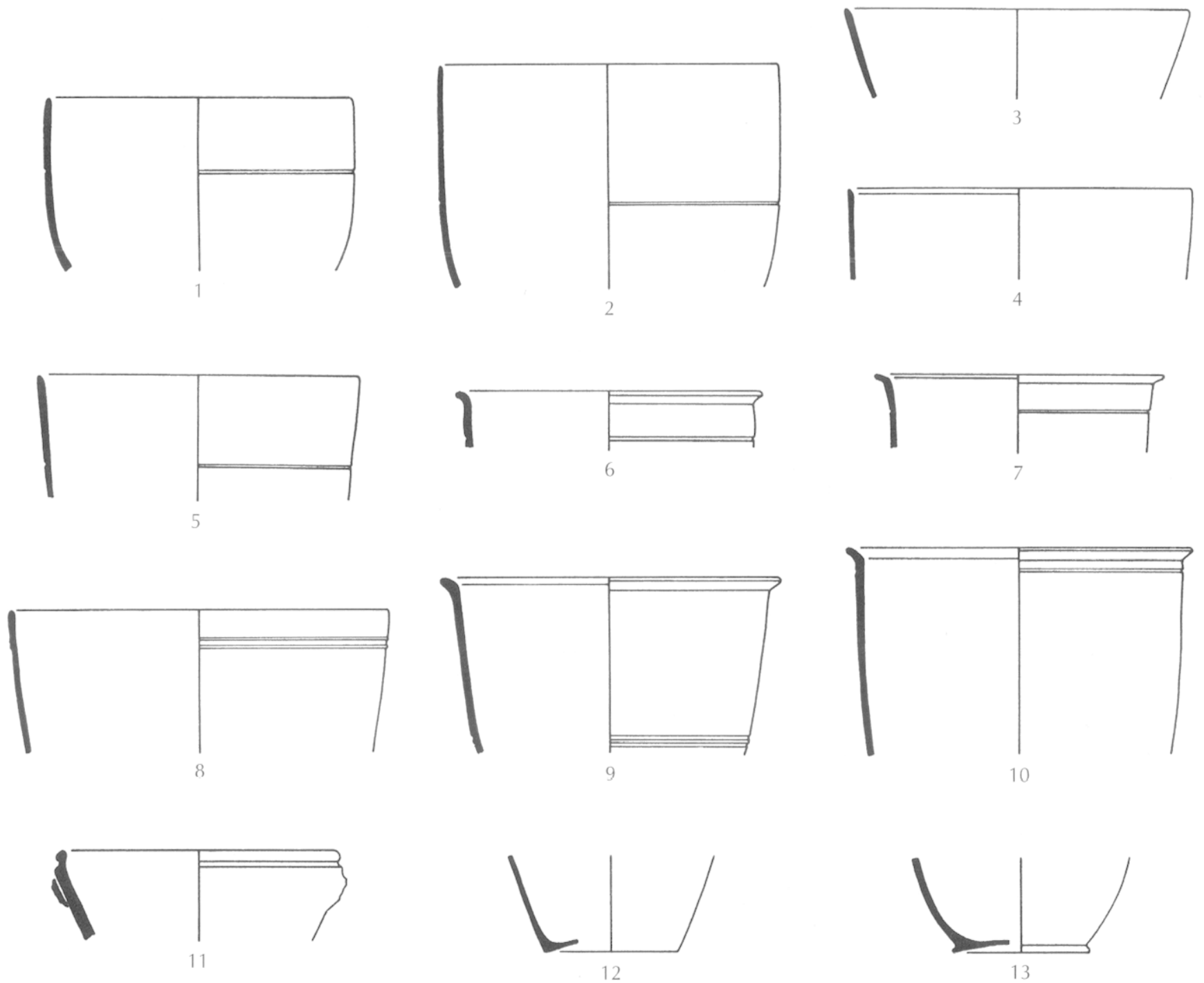
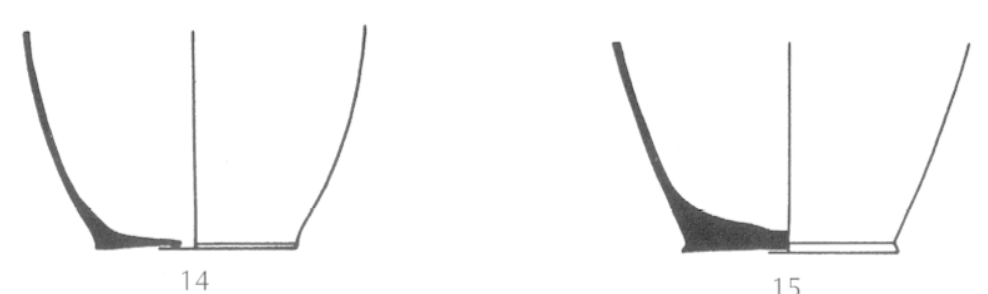

15

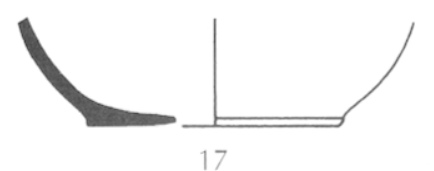

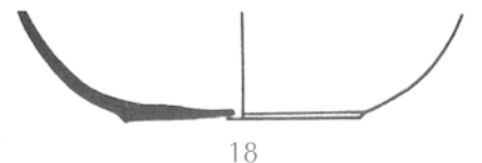

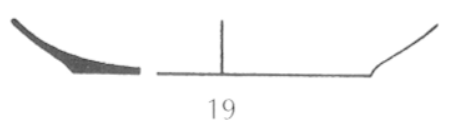

Pl. 38. Einsemble I, valses à parois fines à pâte calcaire (échelle : 1/2). 

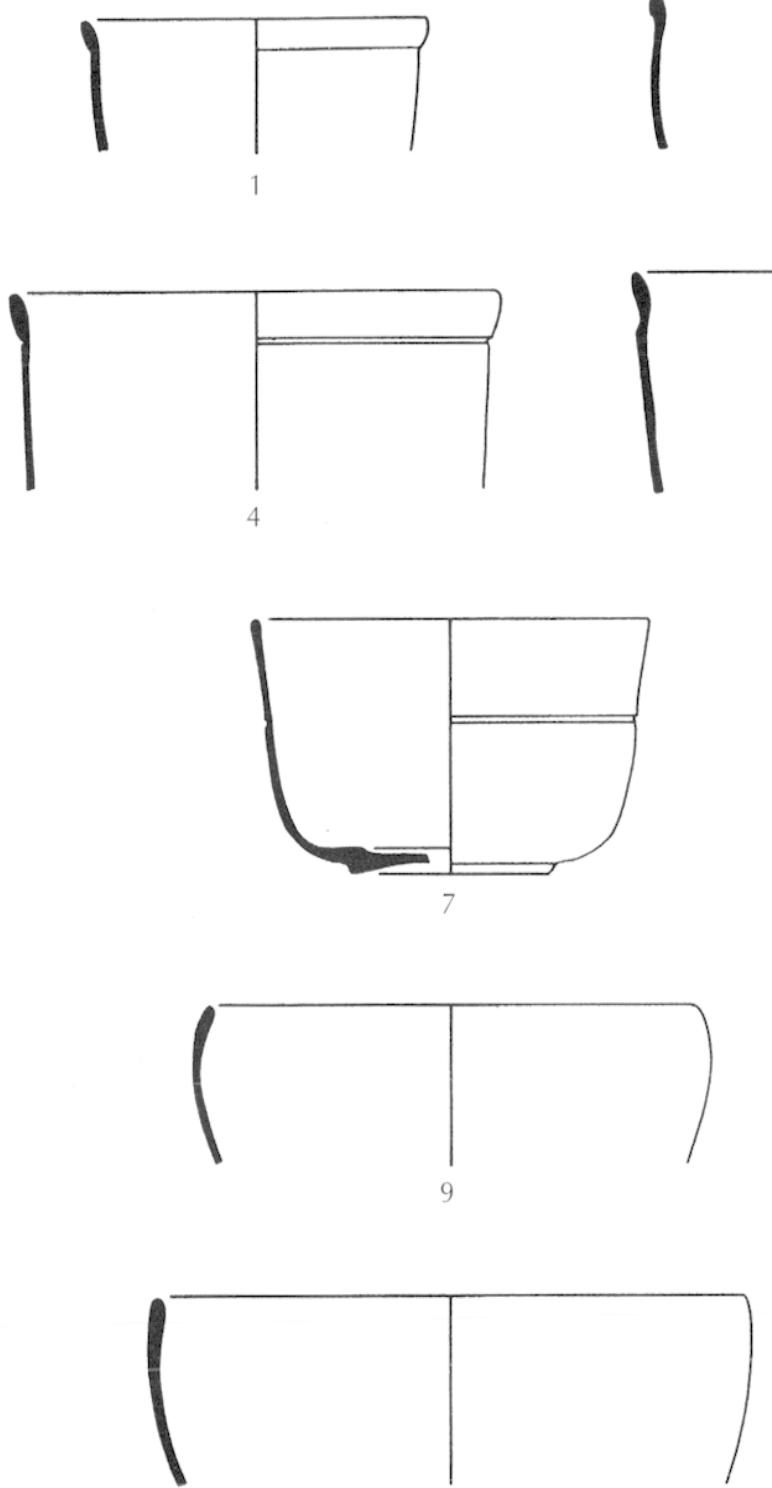

11

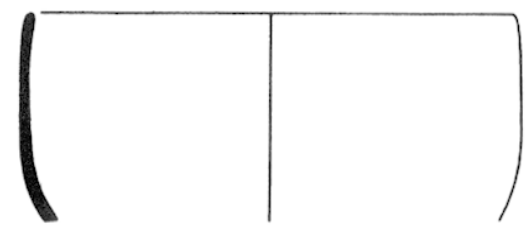

13
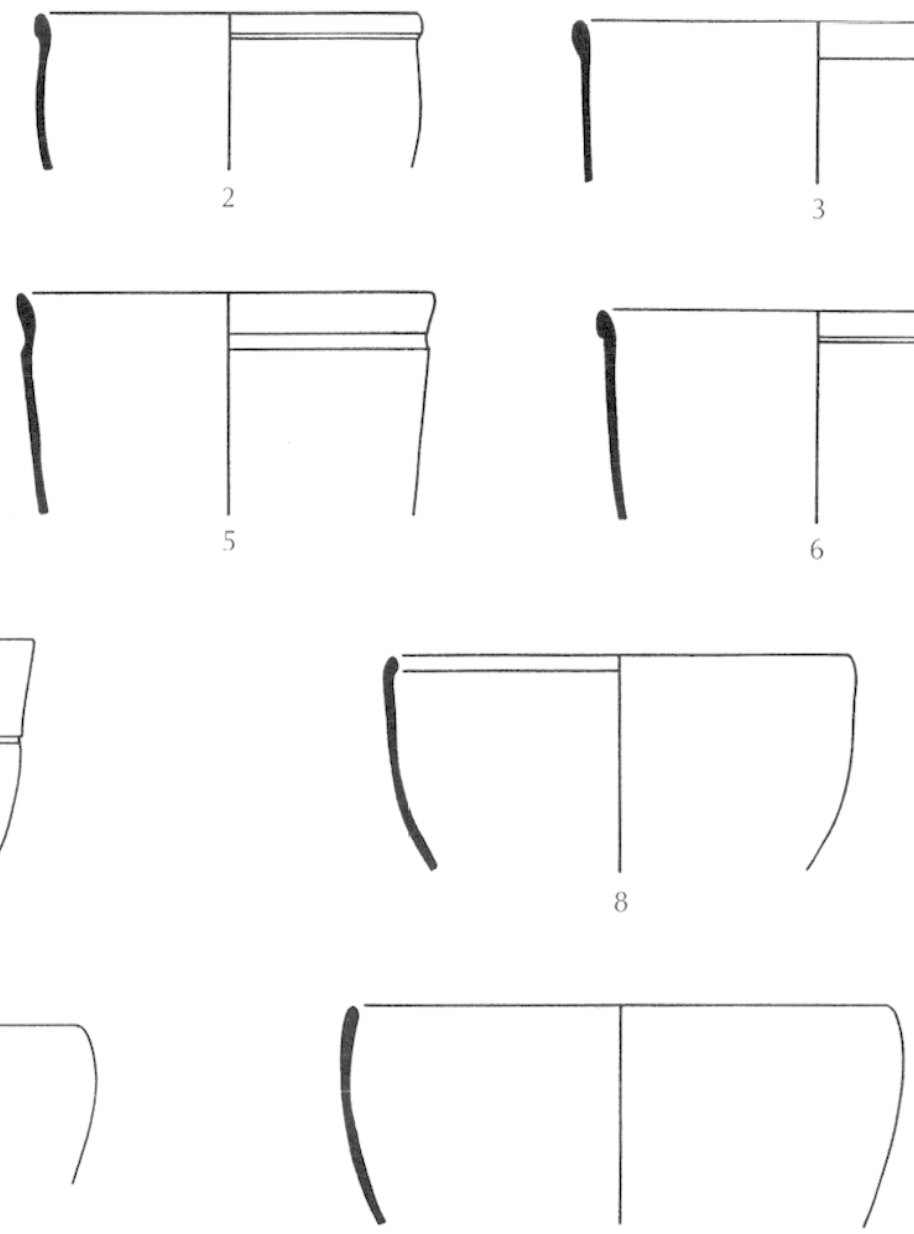

10

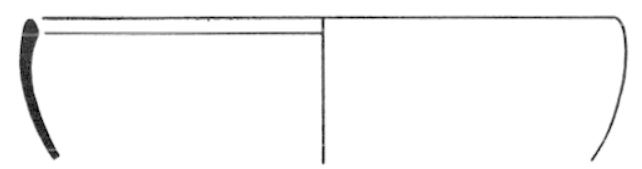

12

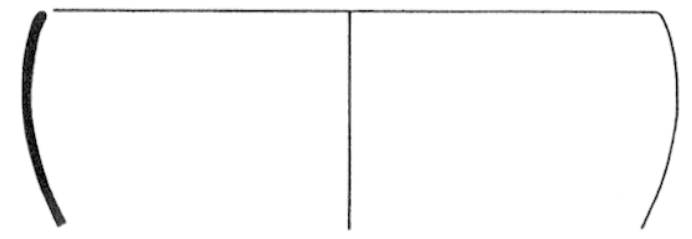

14

P1. 39. Ensemble II, vases à parois fines : gobelets-tonneaux (1-6) et bols hémisphériques (7-14) (échelle : 1/2). 

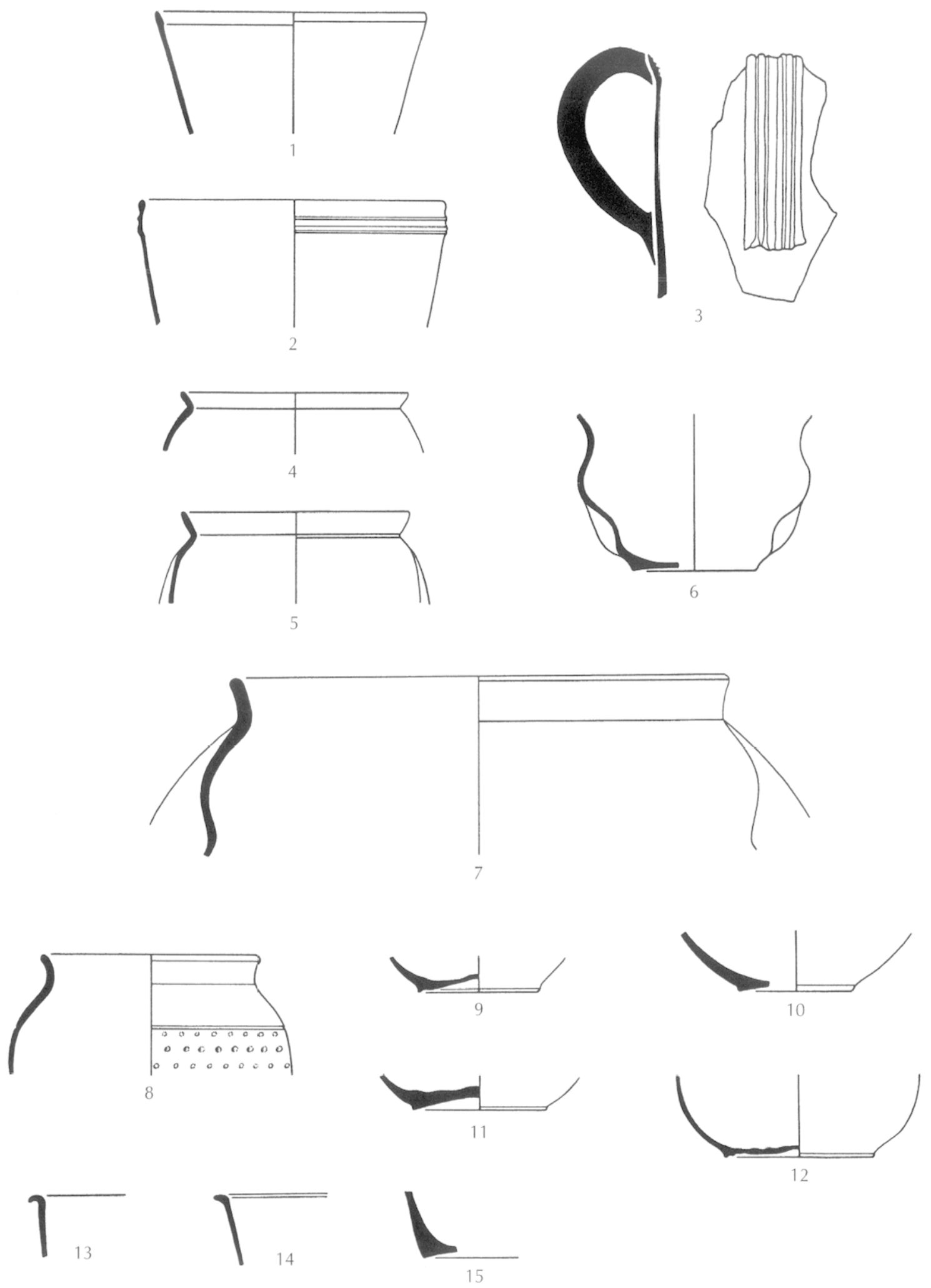

Pl. 40. Ensemble II, vases à parois fines: gobelets à bords striés (1-2), gobelet à anse (3), vases à dépressions (4-7), vase à décor clouté (8), fonds divers (9-12) et vases à pâte calcaire (13-15) (échelle: 1/2). 

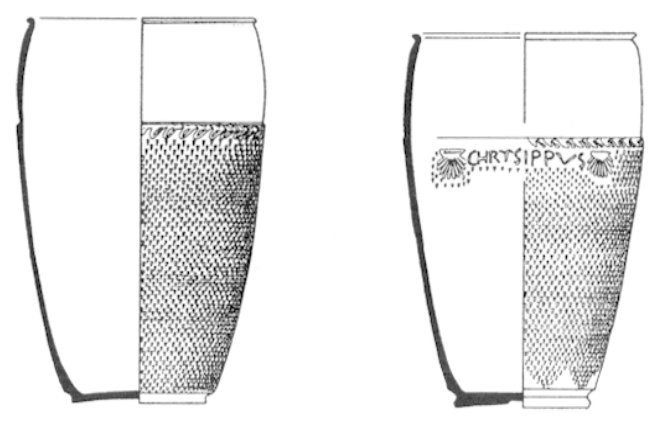

2
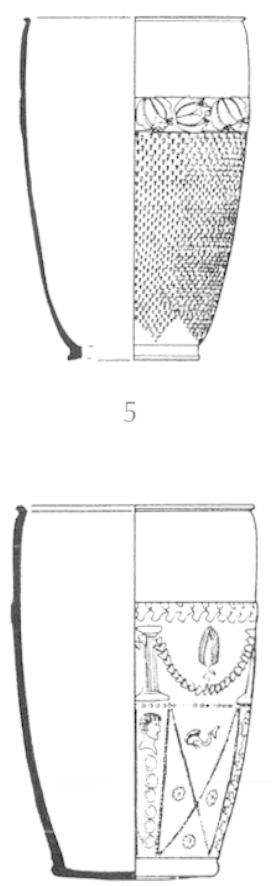

9

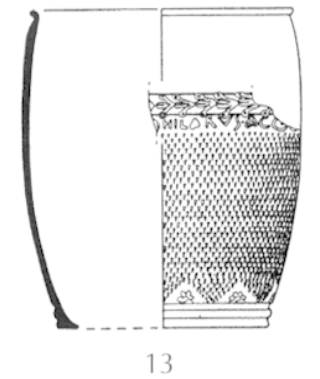

10

14

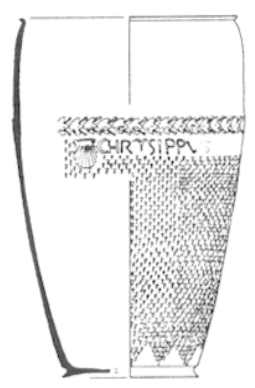

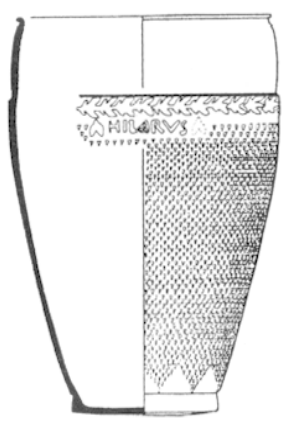

3

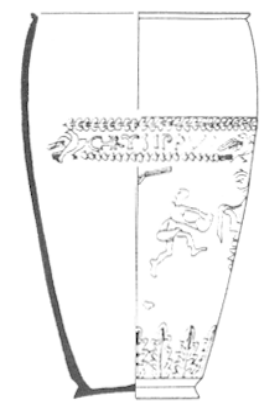

7
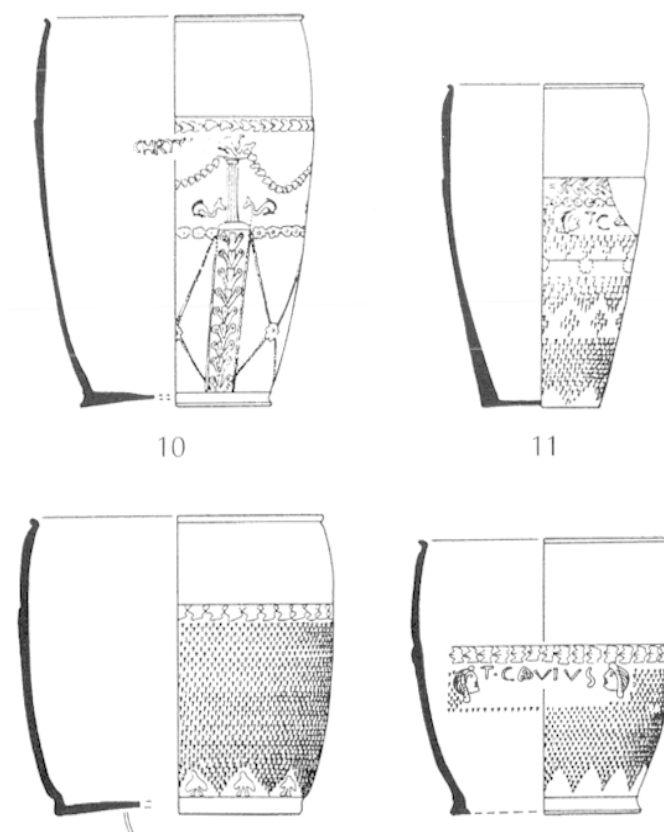

11

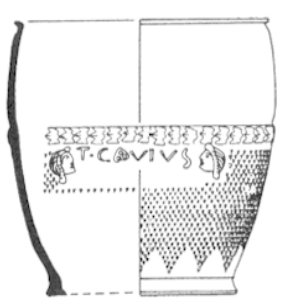

15

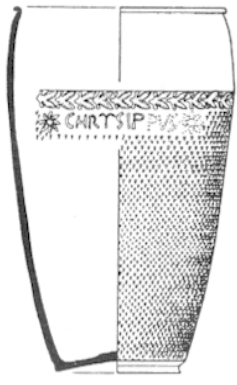

4

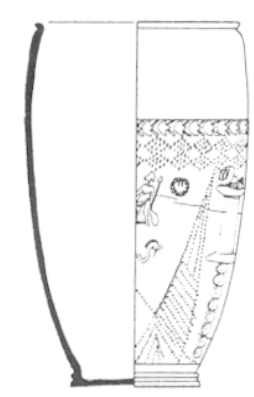

8

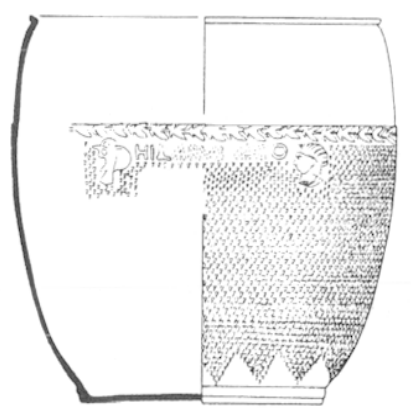

12

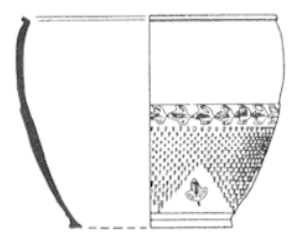

16

P1. 41. Typalogie des gobelets d'Aco: formes hautes (1-12) et formes basses (13-16) (échelle: 1/3). 


\begin{tabular}{|c|c|c|c|c|c|c|c|c|c|c|c|c|c|c|c|c|c|}
\hline & & \& & 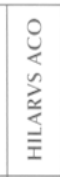 & 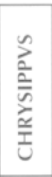 & 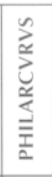 & 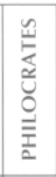 & 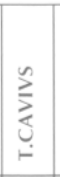 & 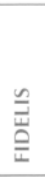 & & & $\gtreqless$ & 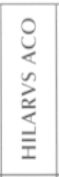 & 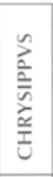 & 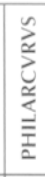 & 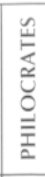 & & $\cong$ \\
\hline 1 & & & $\bullet$ & $\bullet$ & & & & & 15 & & & $\bullet$ & & & $\bullet$ & & \\
\hline 2 & & & & & $\bullet$ & & & & 16 & & & & & & & & \\
\hline 3 & $19,1,3, y, 3$, & & $\bullet$ & & & & & & 17 & & & & $\bullet$ & & & & \\
\hline 4 & & & & & & & $\bullet$ & & 18 & & & & $\bullet$ & & & & \\
\hline 5 & & & & & & & ? & & 19 & & & & $\bullet$ & & & & \\
\hline 6 & & & & $\bullet$ & & & & & 20 & & & & $\bullet$ & & & & \\
\hline 7 & -3 & & & $\bullet$ & & & & & 21 & & & $\bullet$ & $\bullet$ & & & & \\
\hline 8 & $34 \operatorname{sen}-2$ & & $\bullet$ & & & & & & 22 & Fed 1 & & & $\bullet$ & & & & \\
\hline 9 & & & $\bullet$ & & & & & & 23 & & & • & & & & & \\
\hline 10 & & & & • & $\bullet$ & & & & 24 & & & $\bullet$ & & & & & \\
\hline 11 & & & & $\bullet$ & & & & & 25 & $-5+\infty \frac{1}{2}=$ & & • & & & & & \\
\hline 12 & 1. $v^{3}+3$ & & $\bullet$ & & & & & & 26 & & & & $\bullet$ & & & & \\
\hline 13 & & & & $\bullet$ & & & $\bullet$ & & 27 & & & & $\bullet$ & & & & \\
\hline 14 & & & $\bullet$ & $\bullet$ & & & & & 28 & & & & • & & & & \\
\hline
\end{tabular}

P1. 42. Lrises (échelle : $1 / I$ ). 


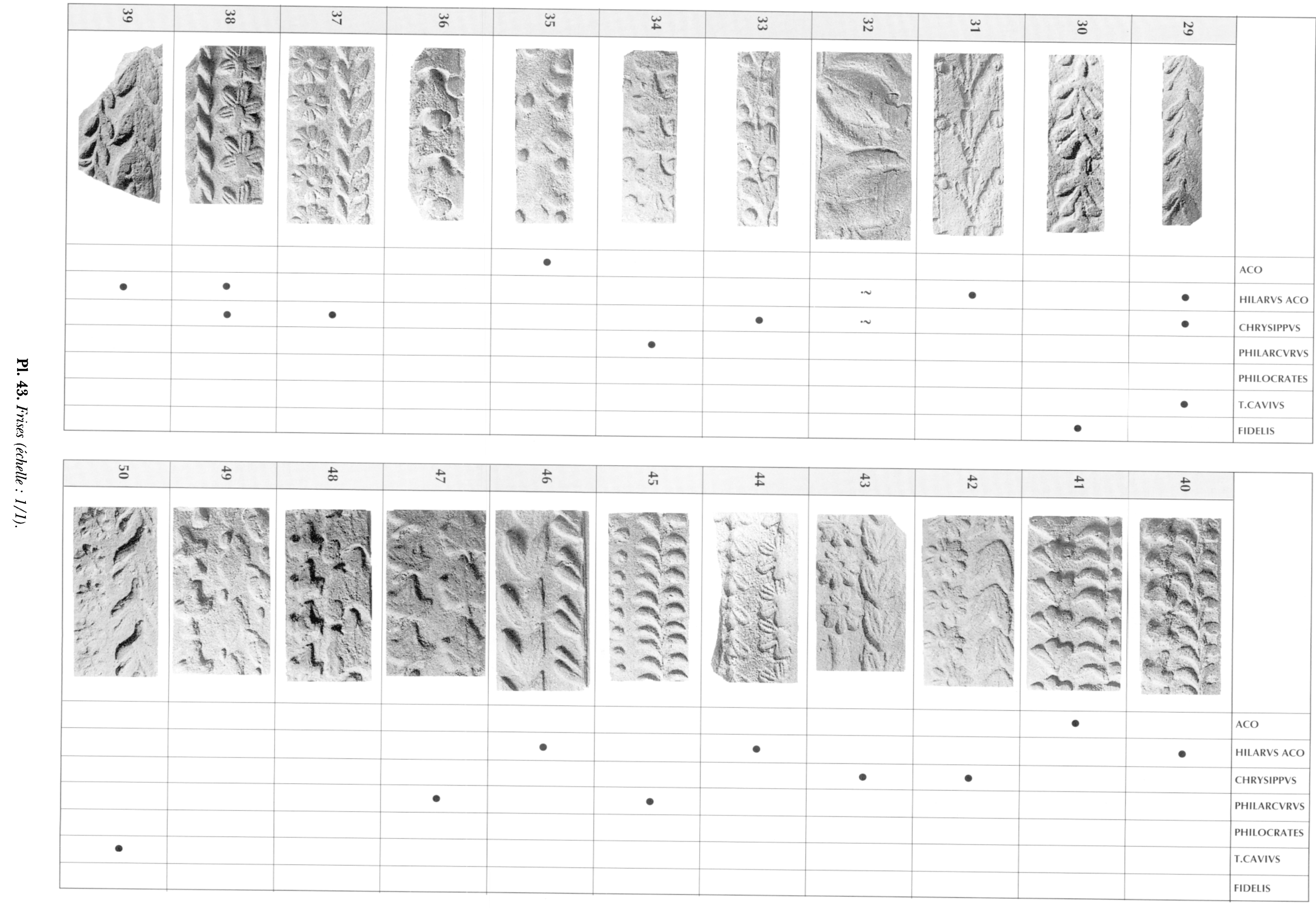




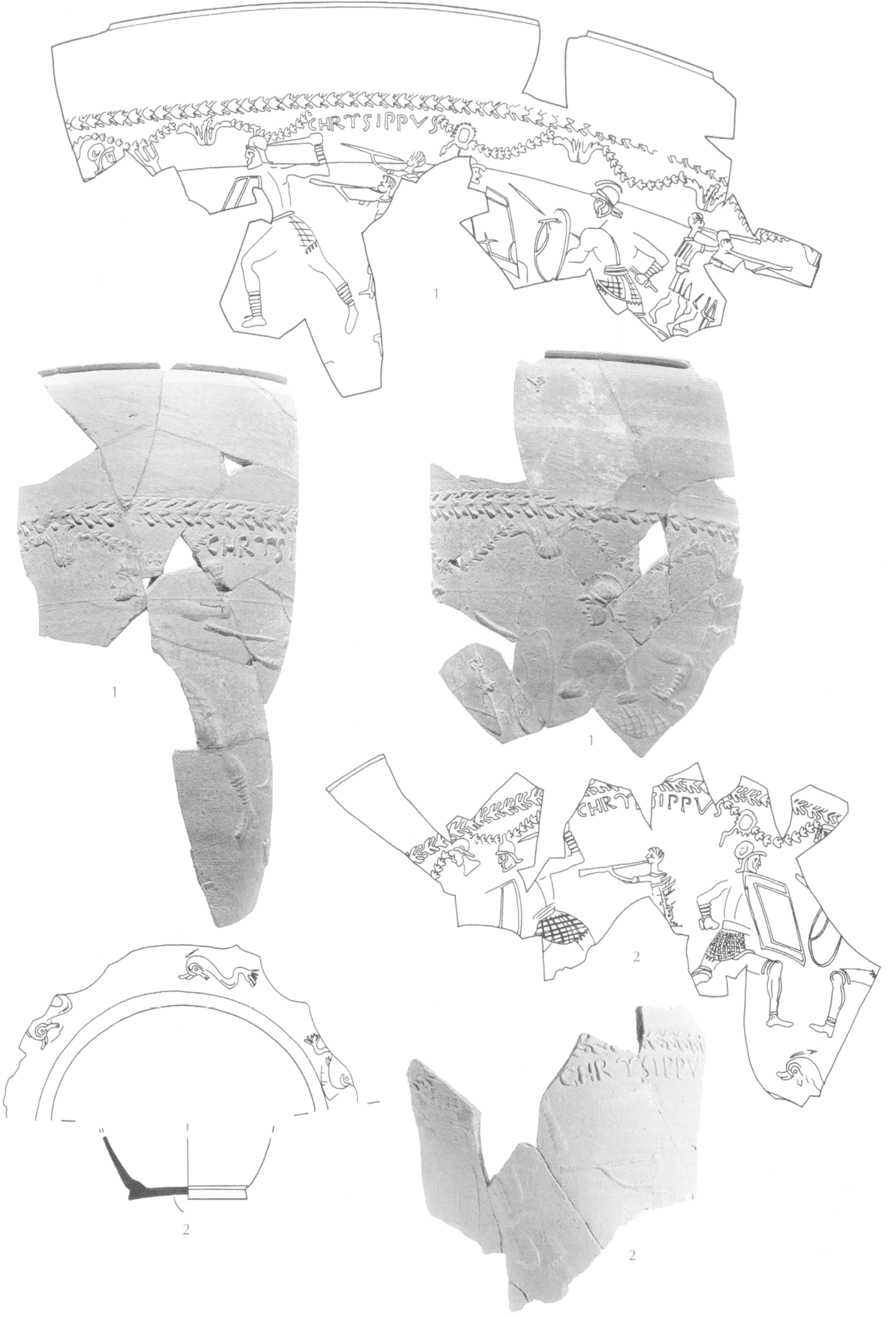

P1. 44. Chnsippres, décors 1 et 2 (échelles dessins: 2/3; photos: 1/1). 


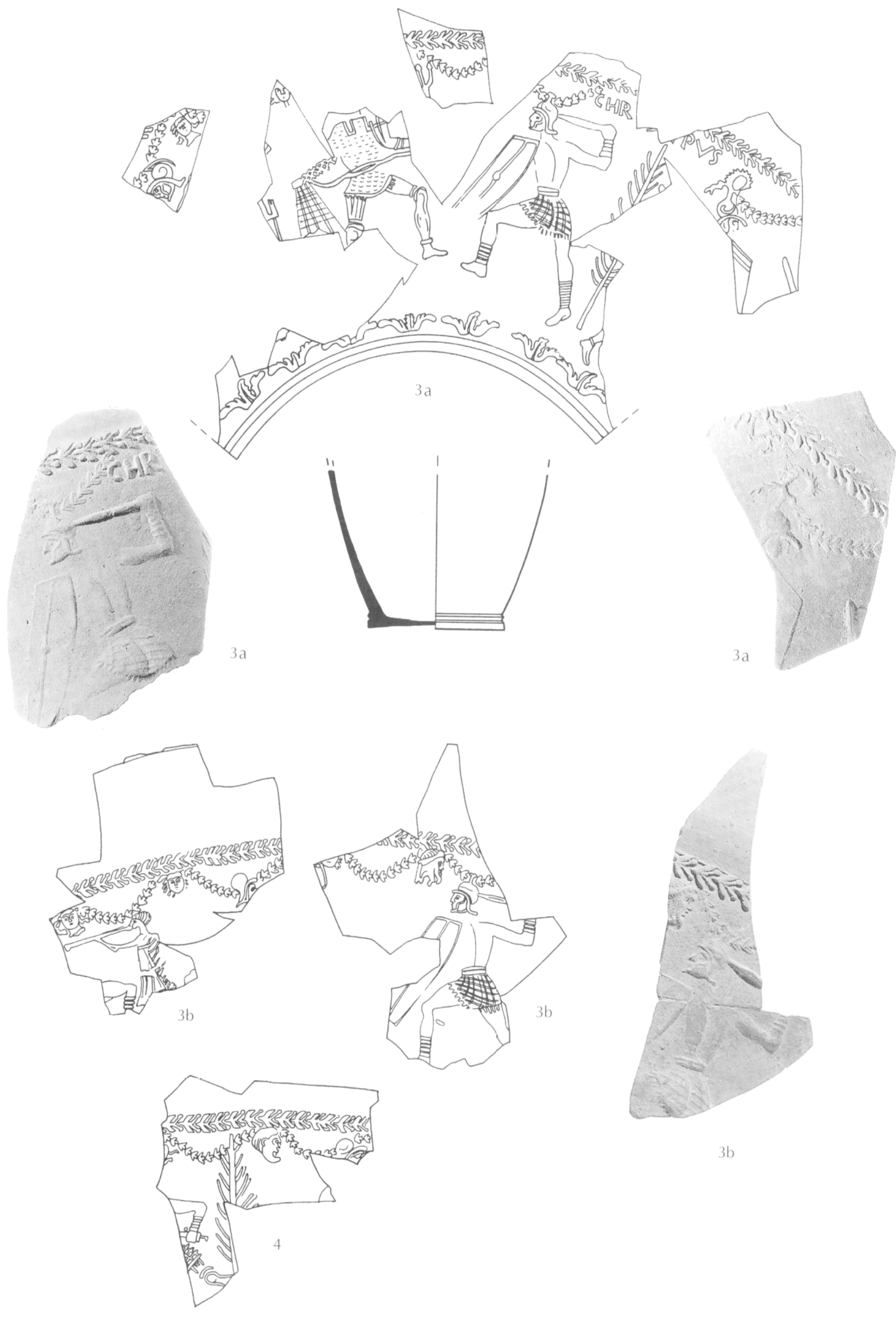

PI. 45. Chrysipprss, décors 3 et 4 (échelles dessins : 2/3; photos : 1/1). 

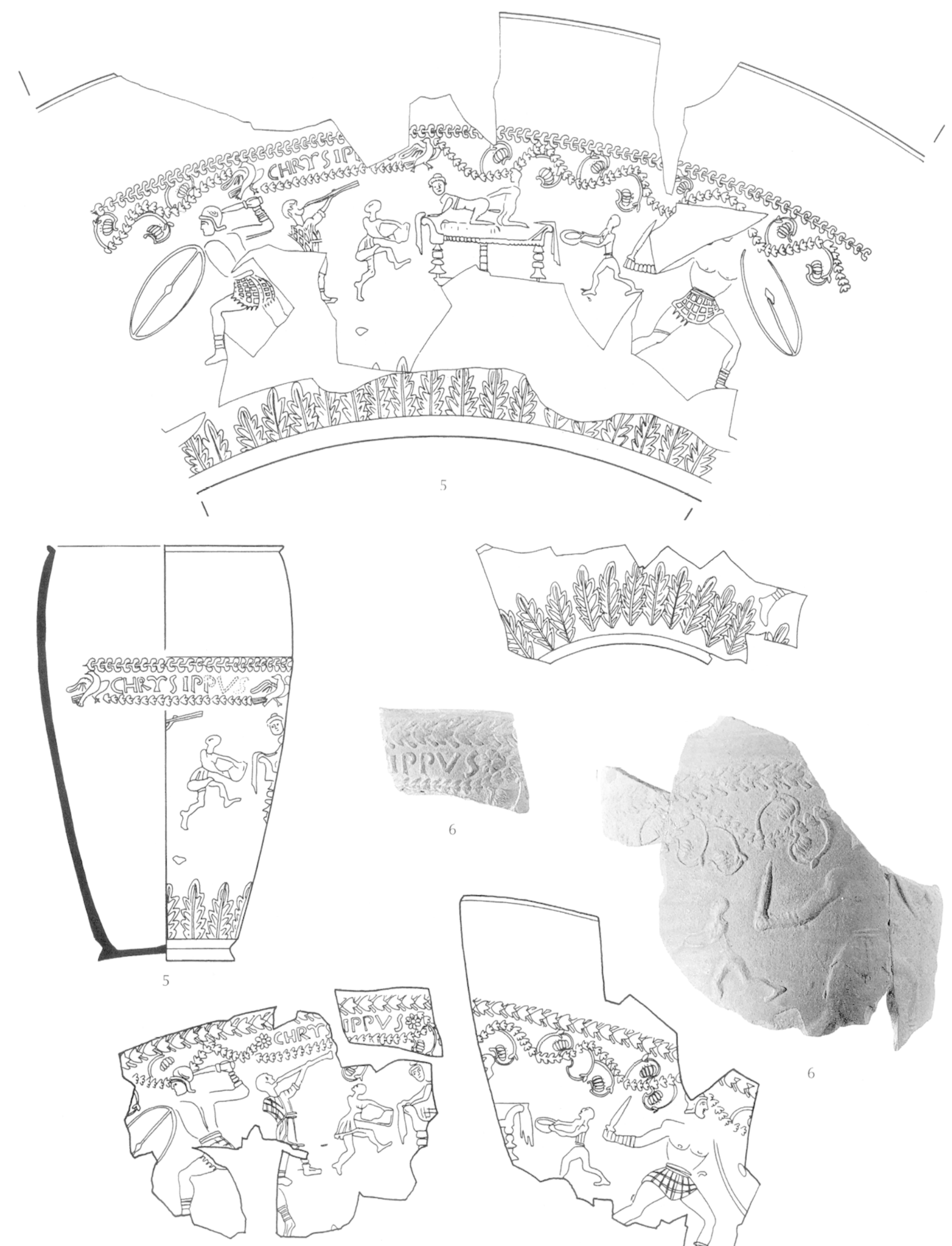

6

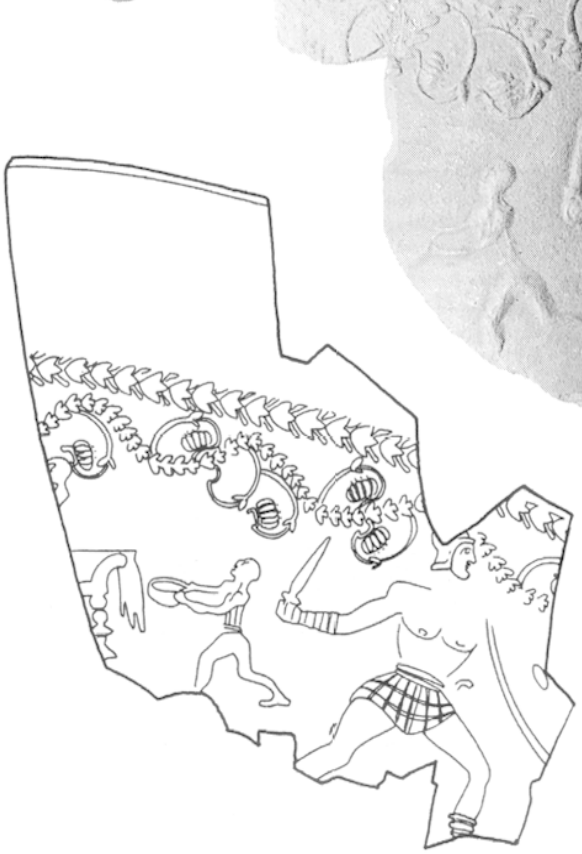

PI. 46. Chrysipprss, décors 5 et 6 (échelles dessins : 2/3; photos : 1/1). 

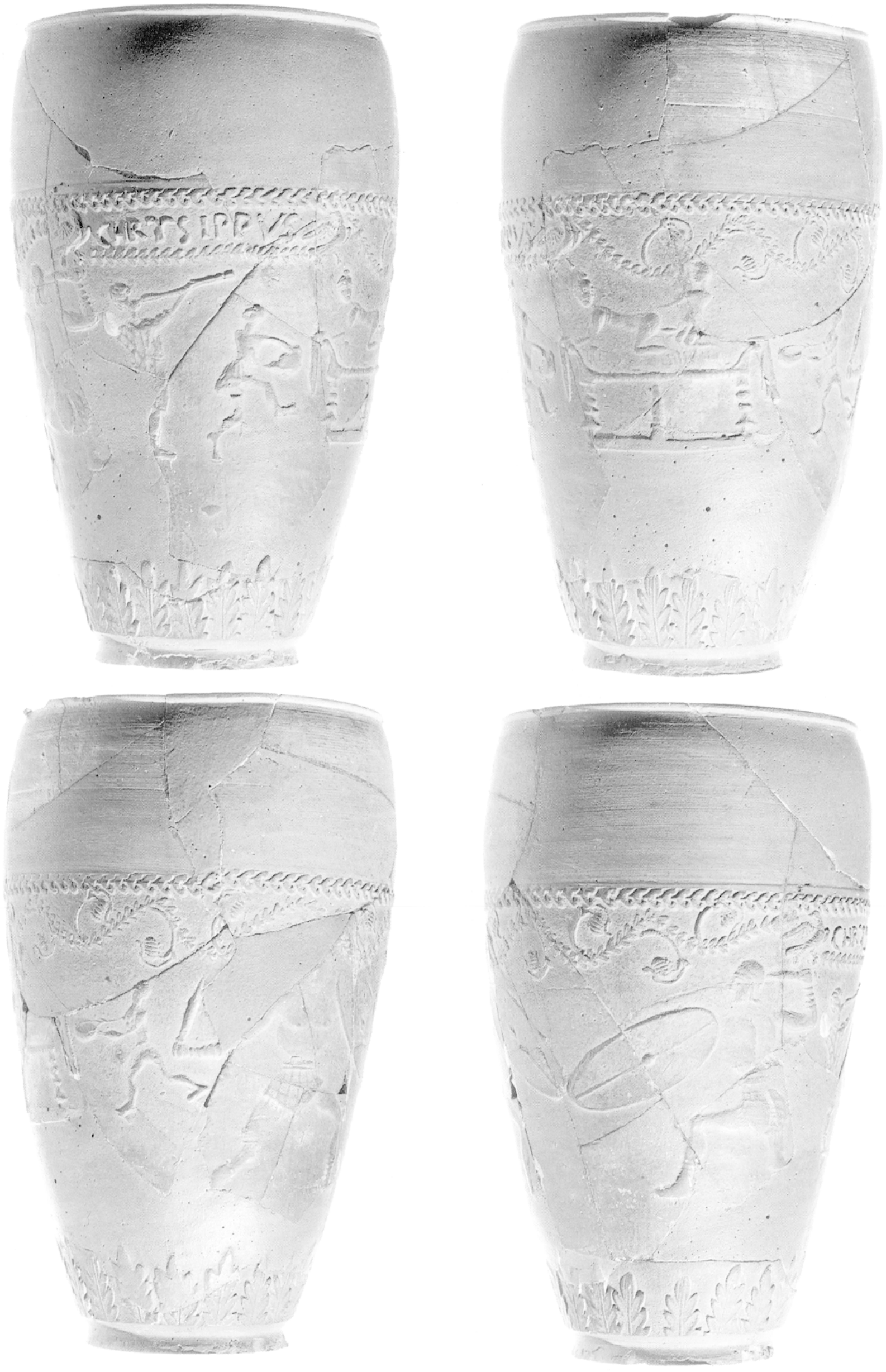

PI. 47. Chyysipprs, décor 5 (échelle : 1/1). 

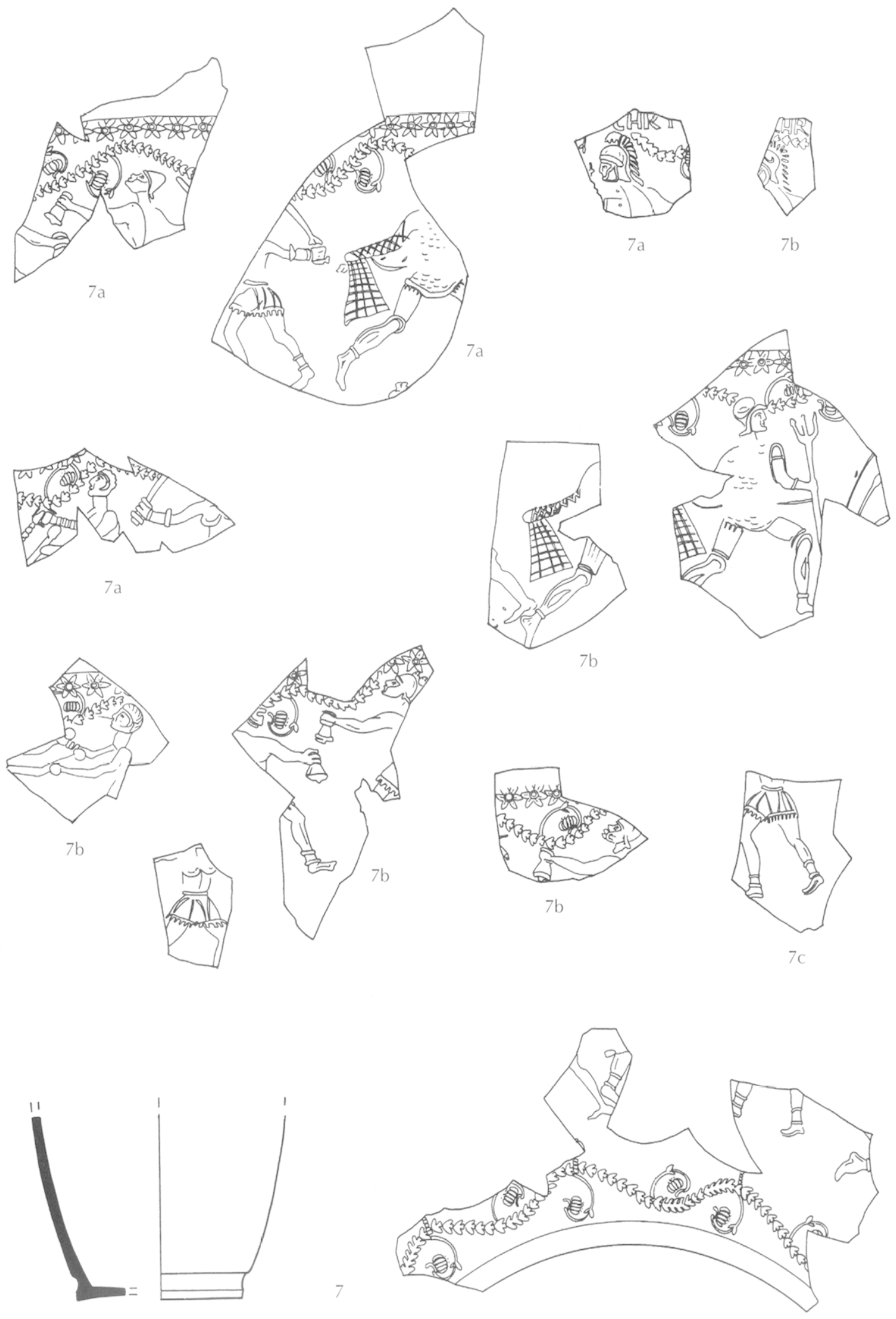

Pl. 48. Chnvsipprss, décor 7 (échelle : 2/3). 


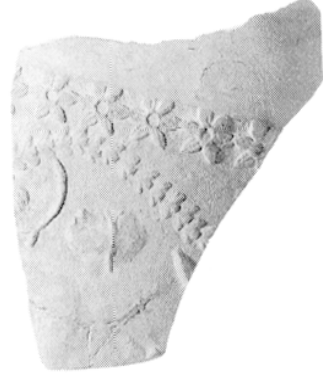

$7 a$

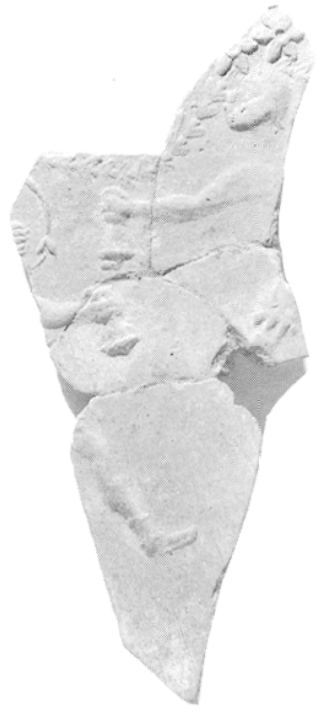

$7 b$

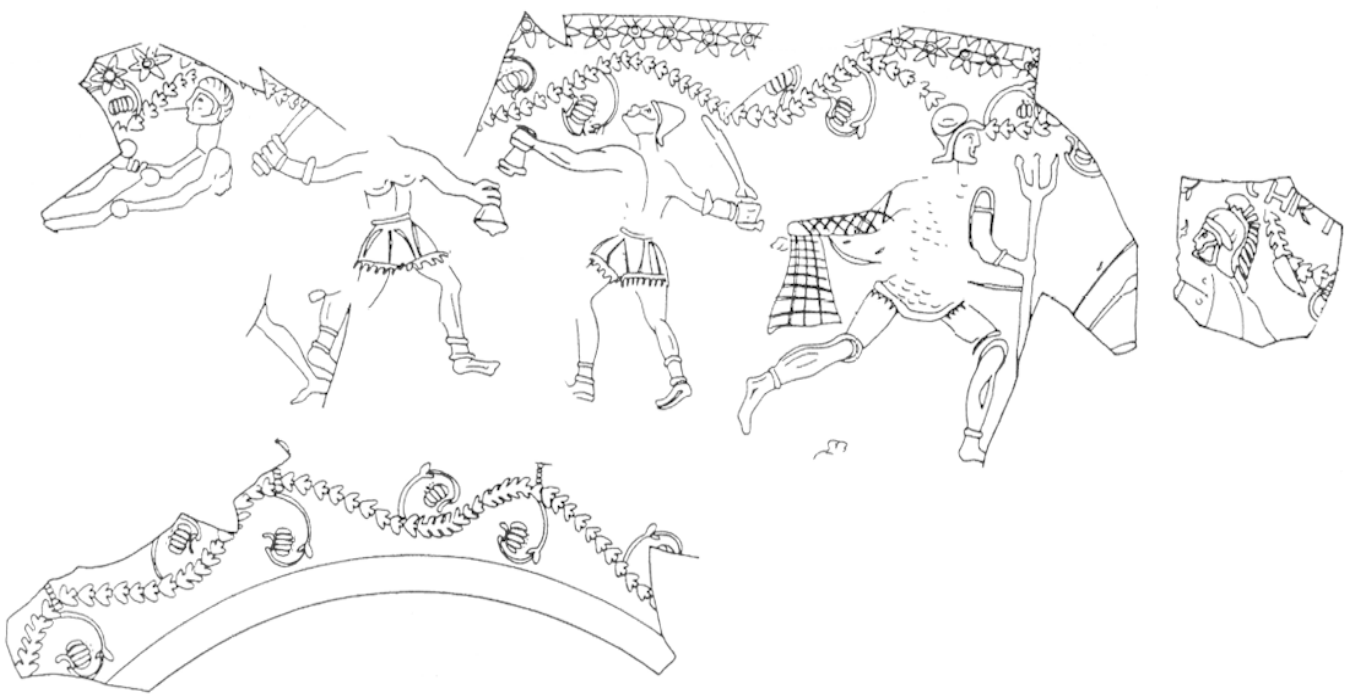

Pl. 49. Chrjsippus, fragments du décor 7 (ésholle : I/l) et reconstitution du décor (échelle : 1/2). 

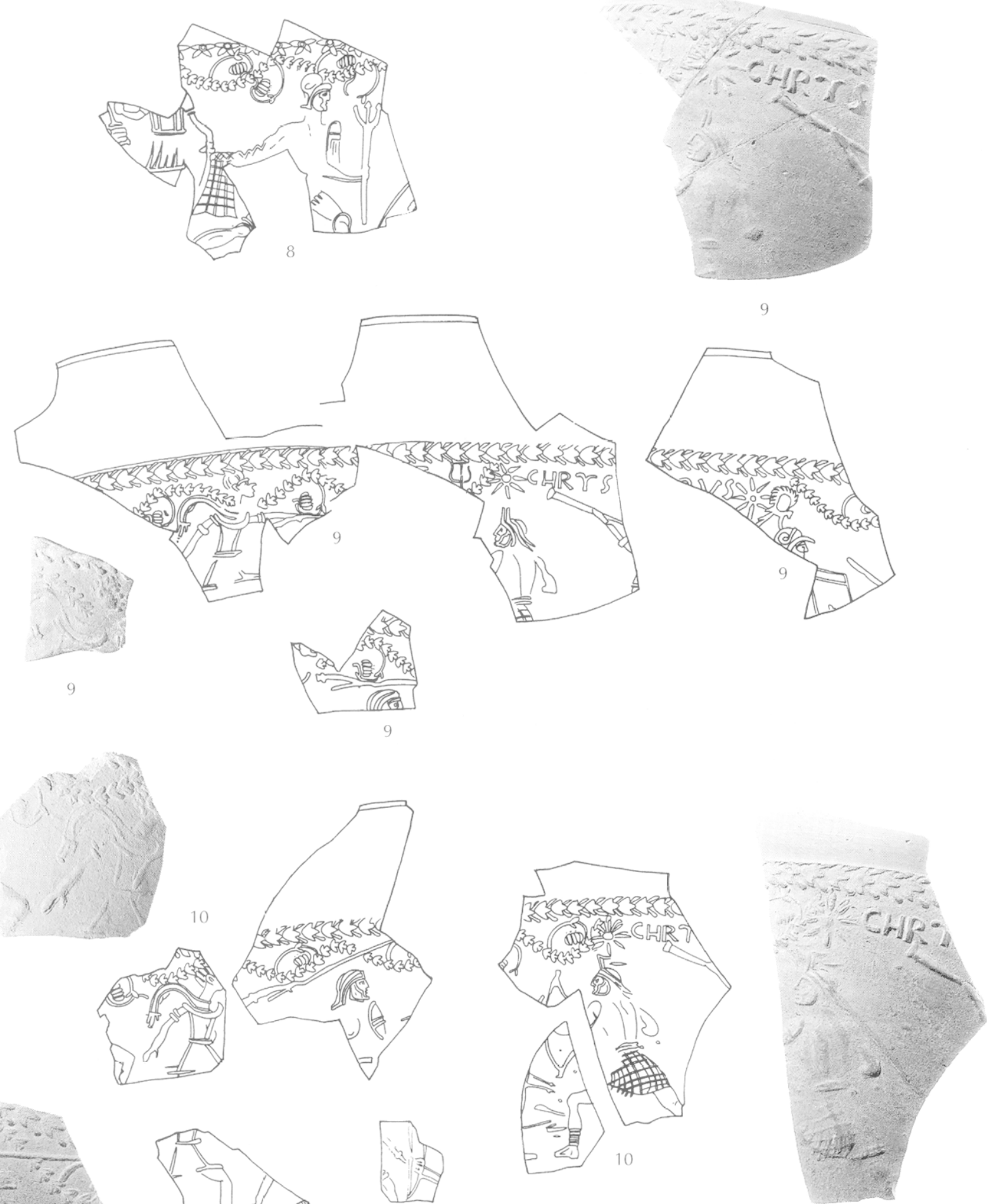

10

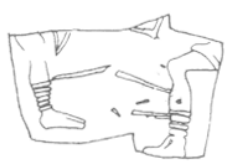

10

Pl. 50. Chnsippres, décors 8 , 9 et 10 (échelles dessins : $2 / 3$; photos : $1 / 1$ ). 

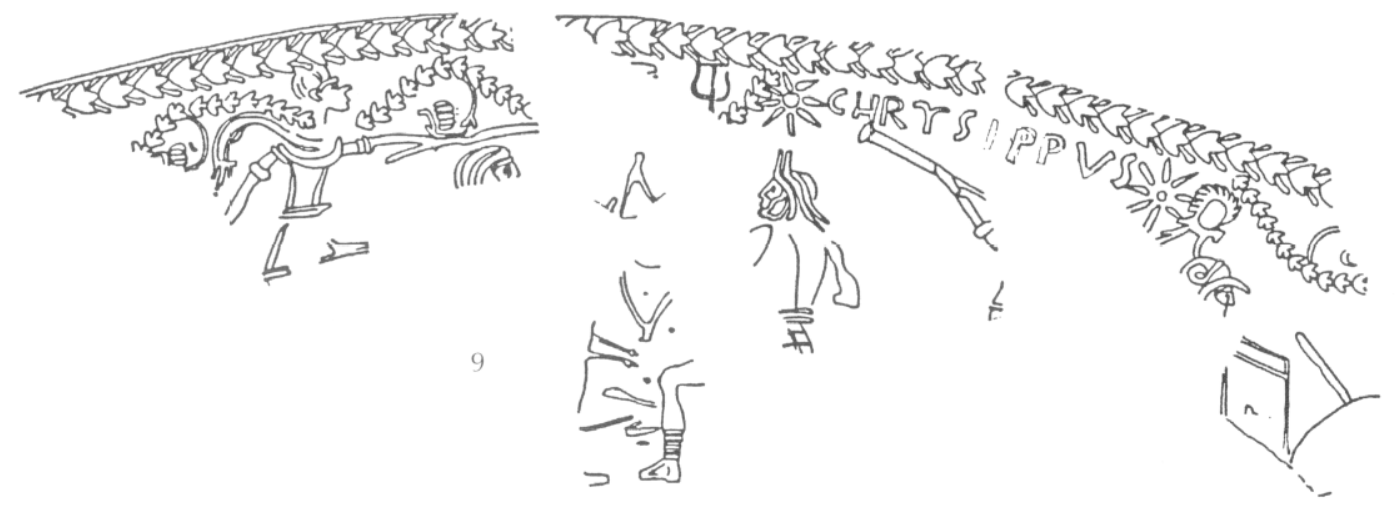
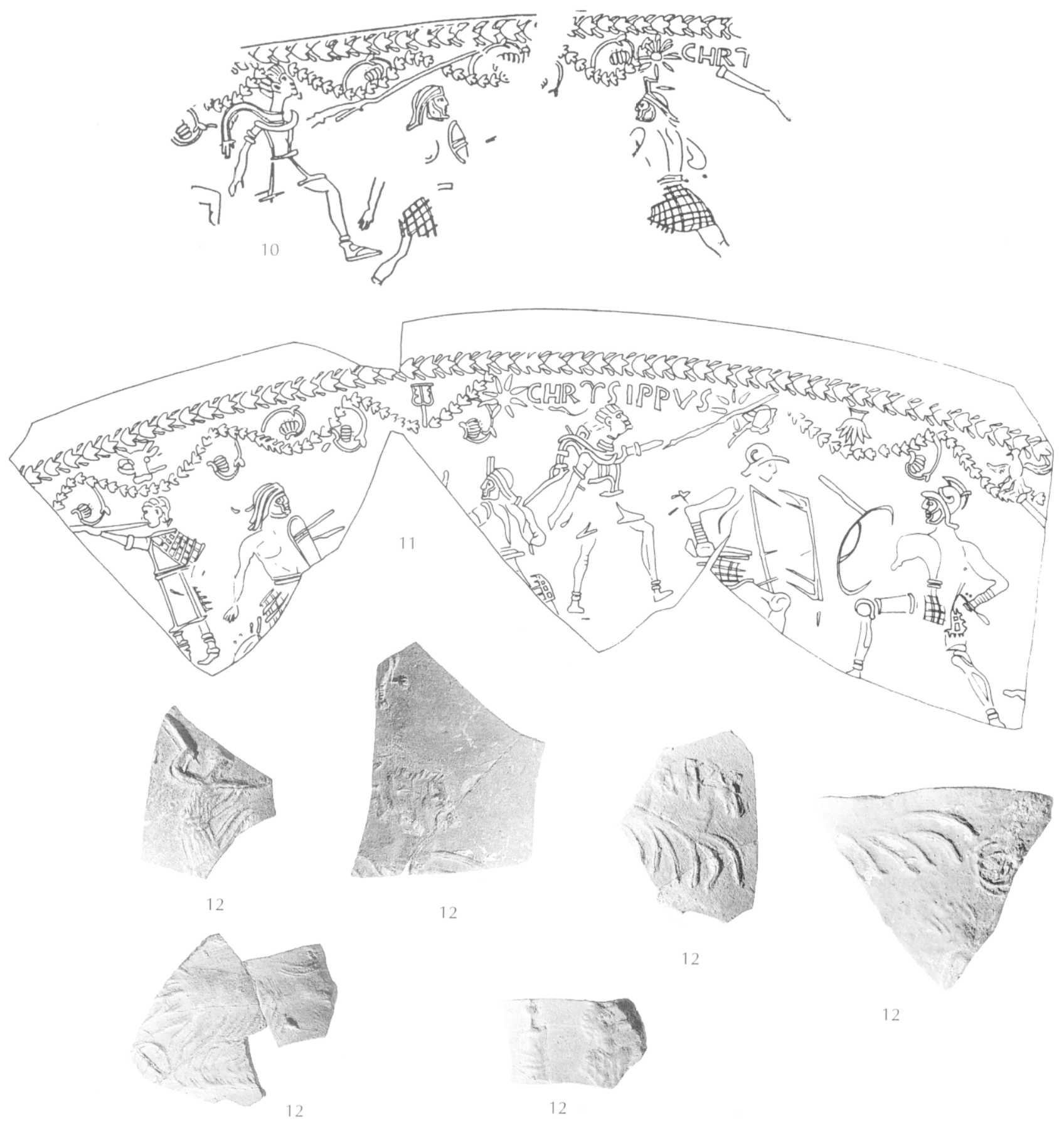

P1. 51. Chnsippros, reconstitution des décors 9, 10 et 11 (échelle: 2/3) et décor 12 (échelle: 1/1). 


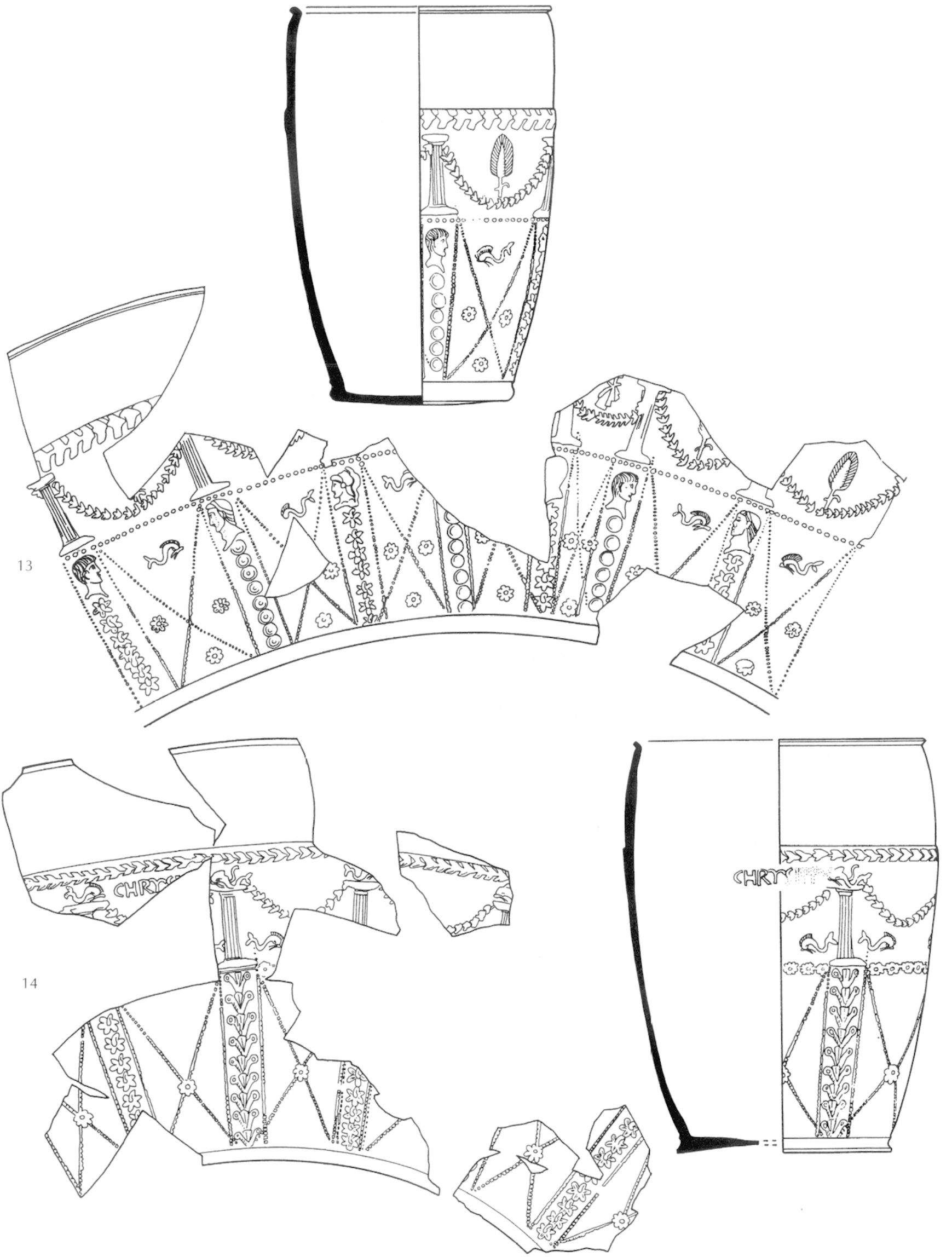

P1. 52. Chrysipprs, décors 13 et 14 (échelle : 2/3). 

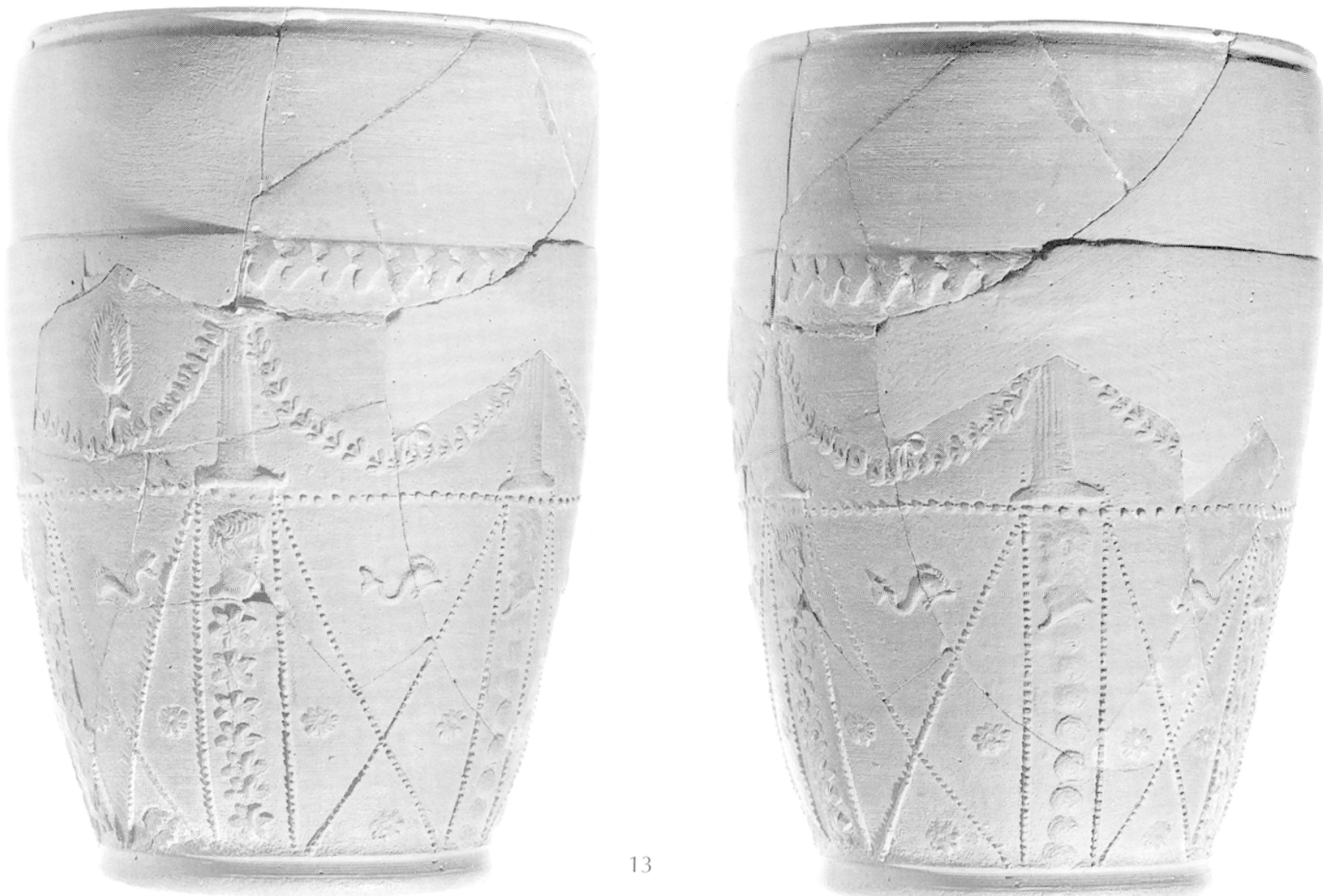

13
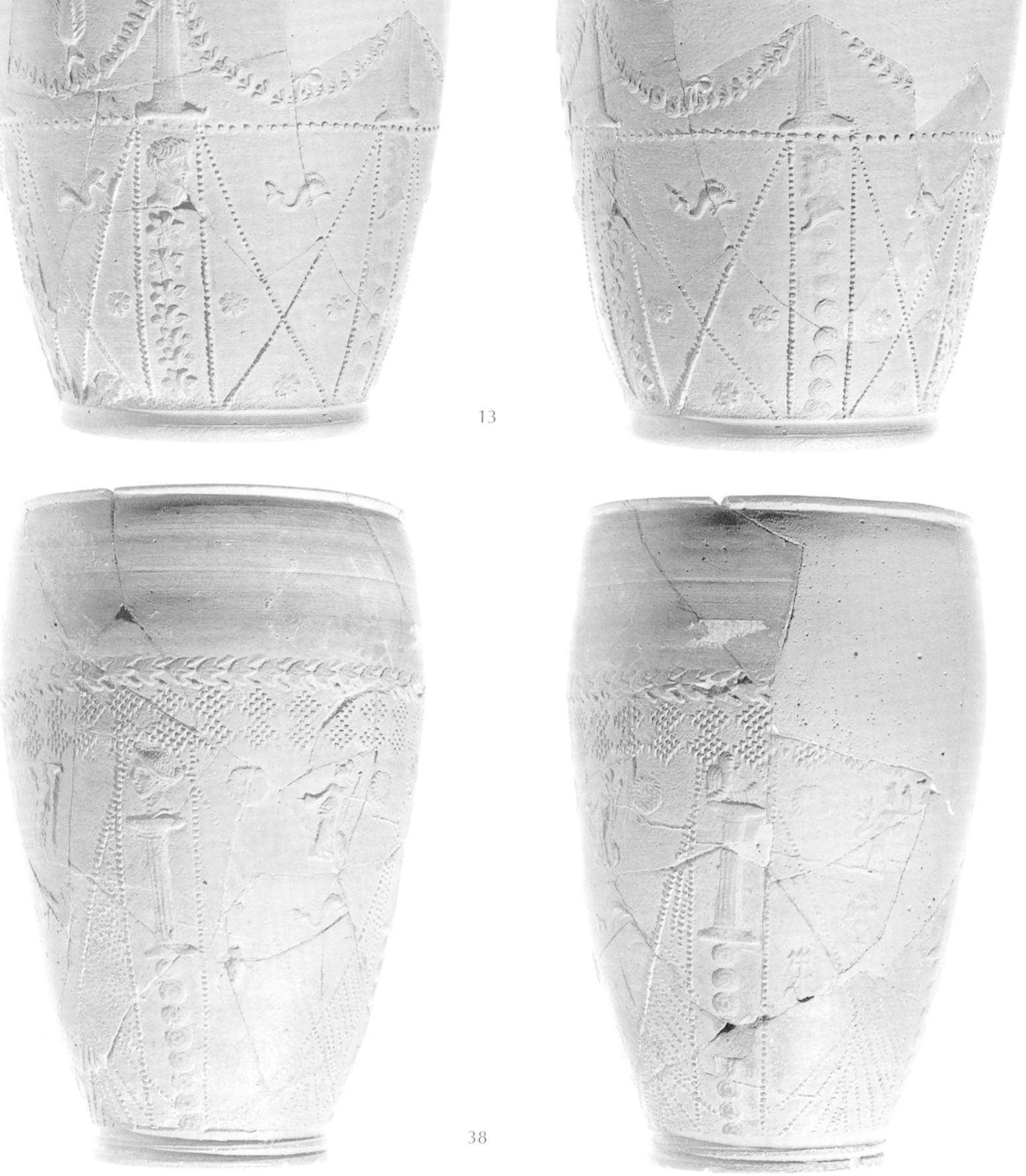

Pl. 53. (hnysippos, décors 13 et 38 (échelle : $1 / 1$ ). 

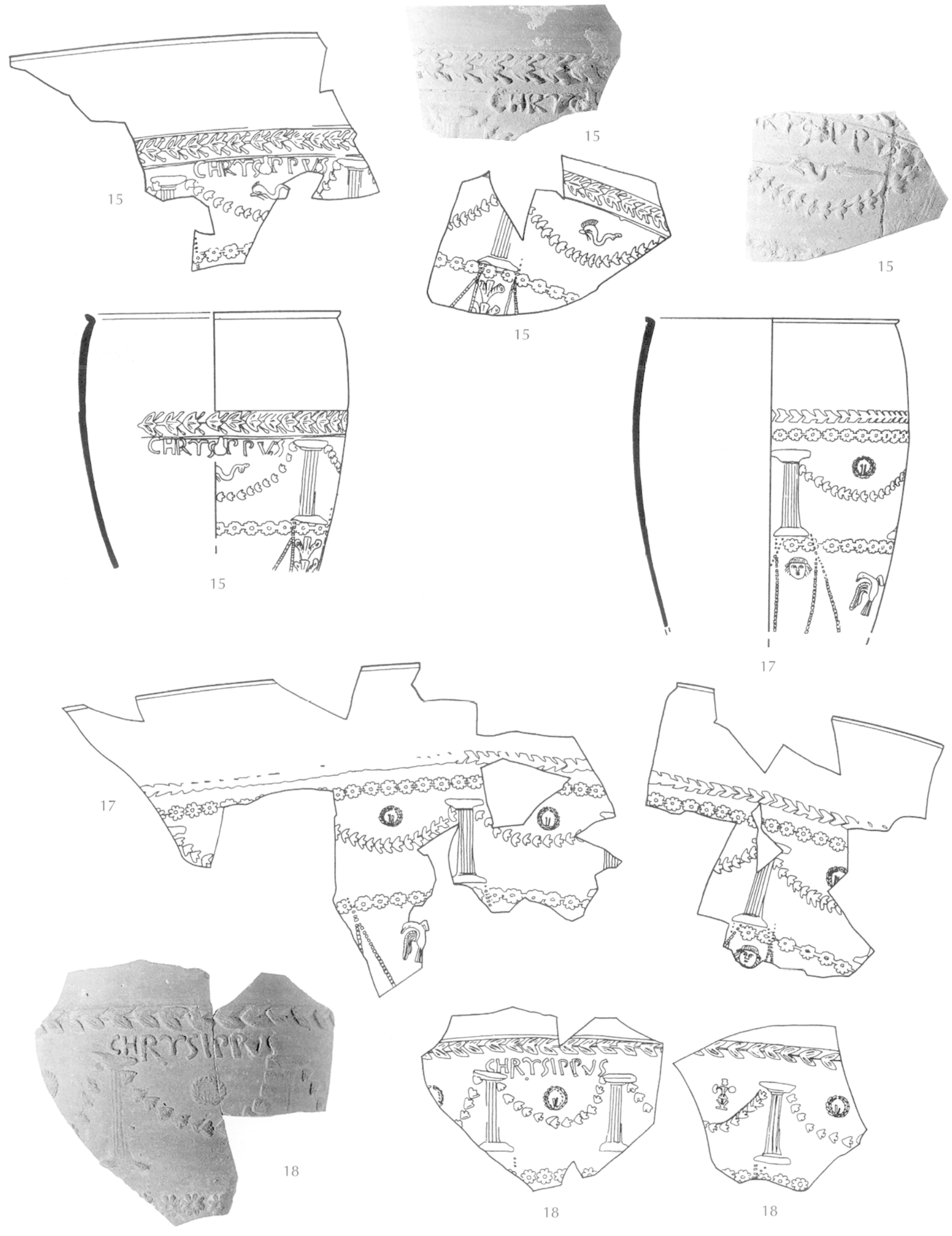

PI. 54. Chnvippris, décors 15,17 et 18 (échelles dessins : $2 / 3$; photos : $1 / 1$ ). 

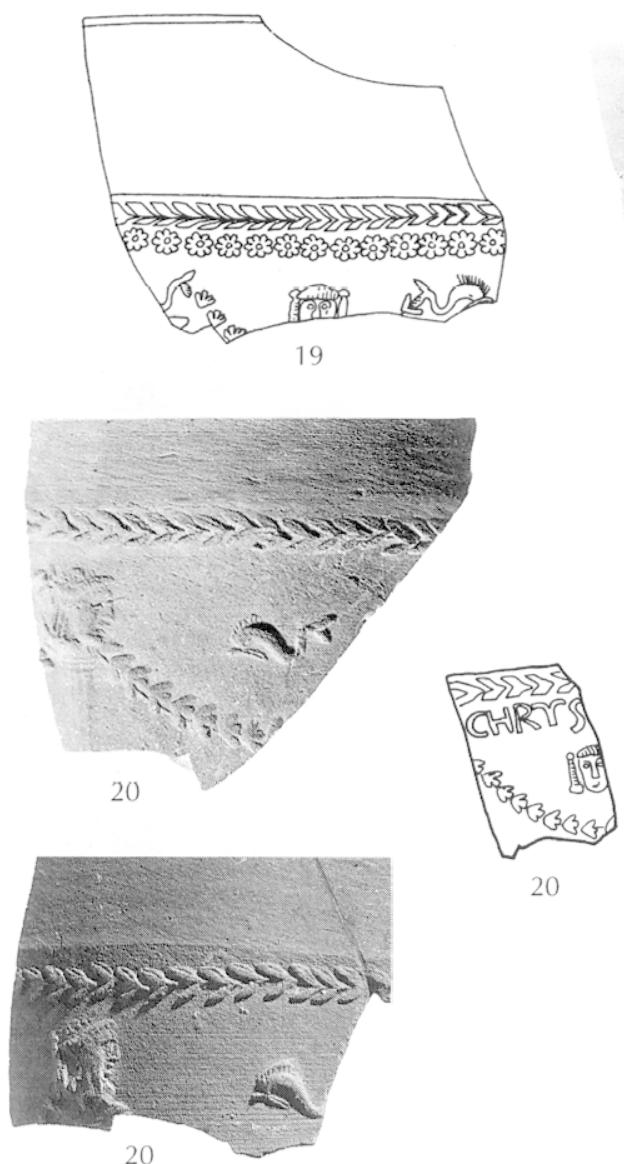

20
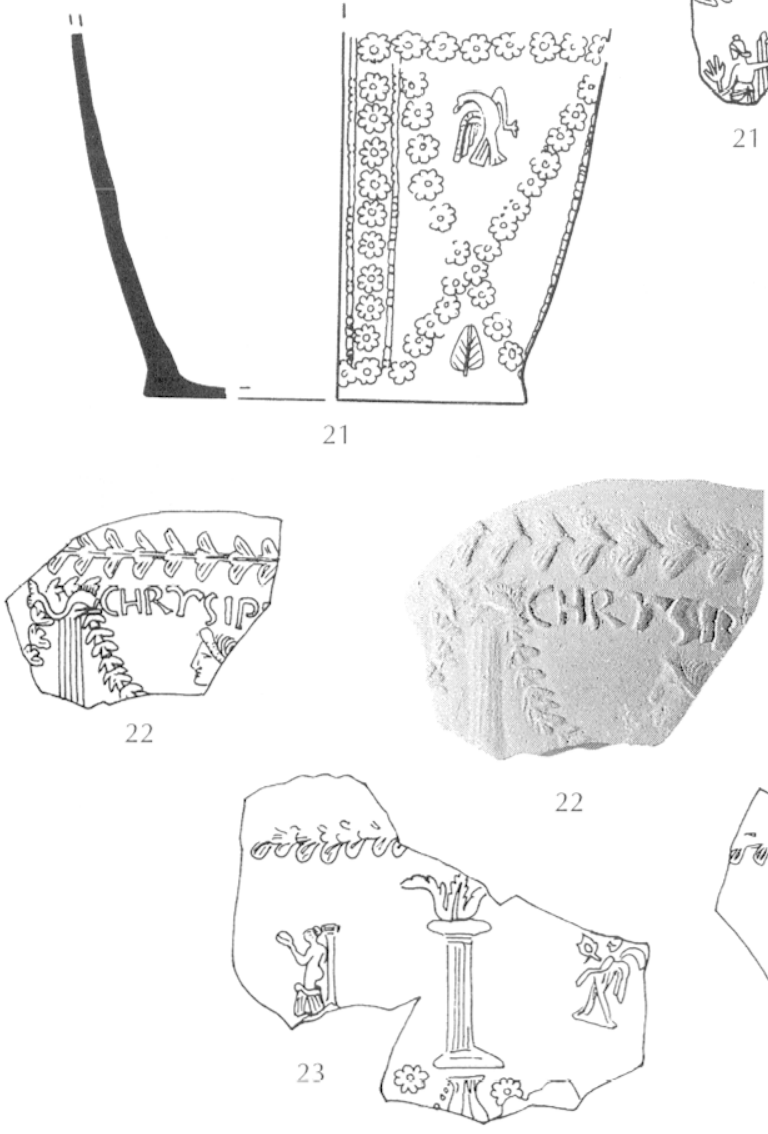

21
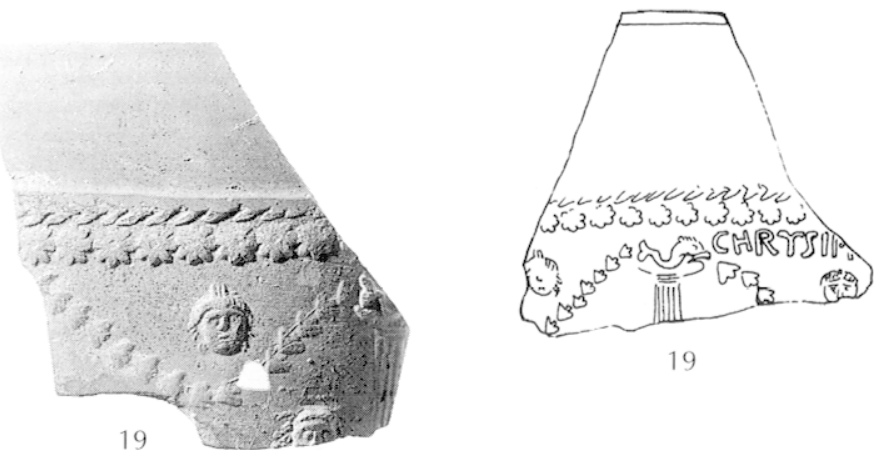

19
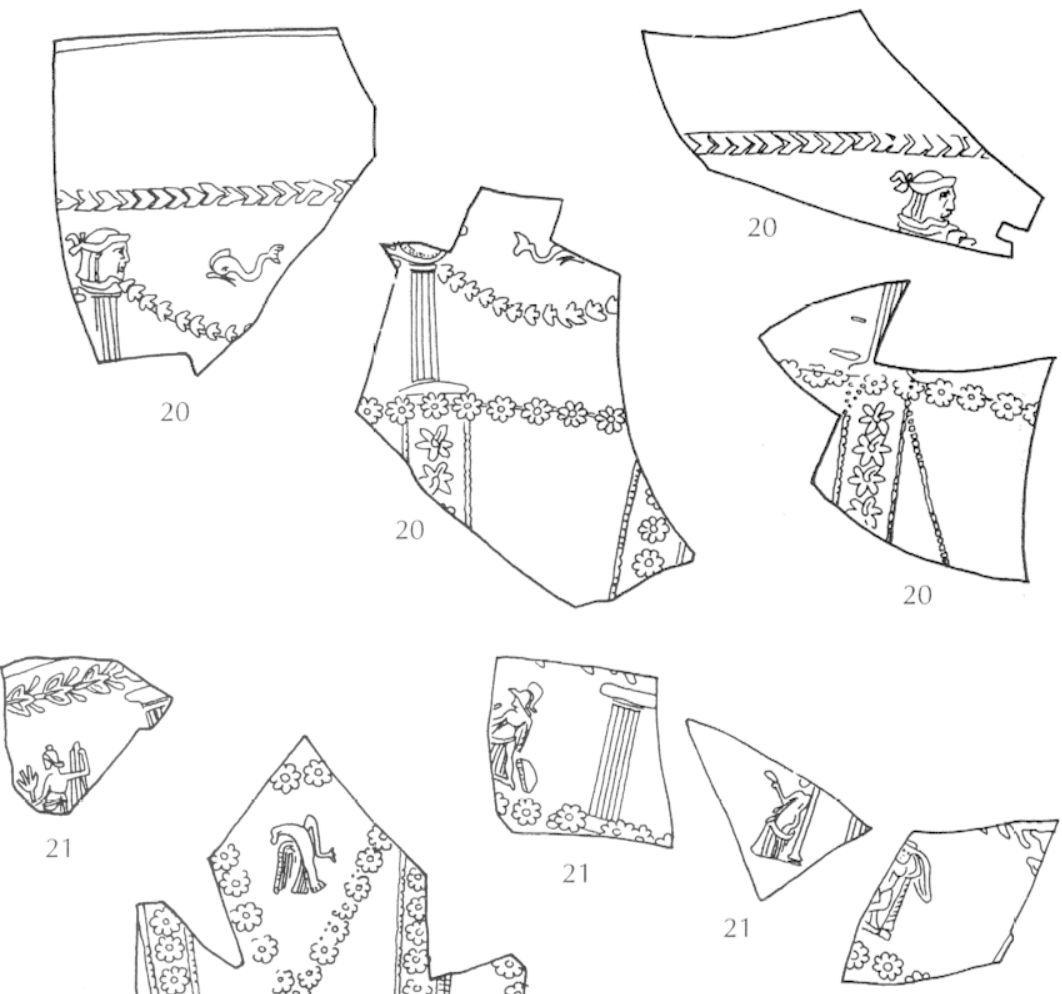

21

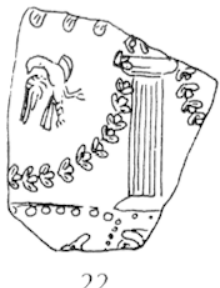

22
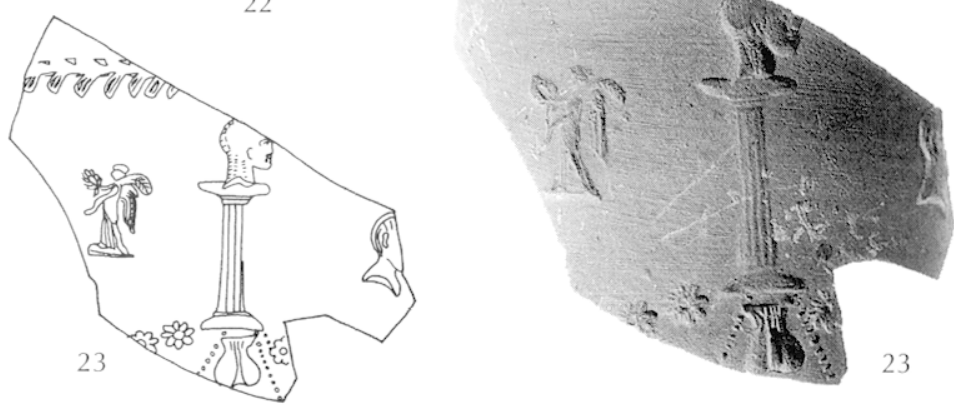

P1. 55. Chnysippros, décors 19, 20, 21, 22 et 23 (échelles dessins : 2/3; photos : 1/1). 

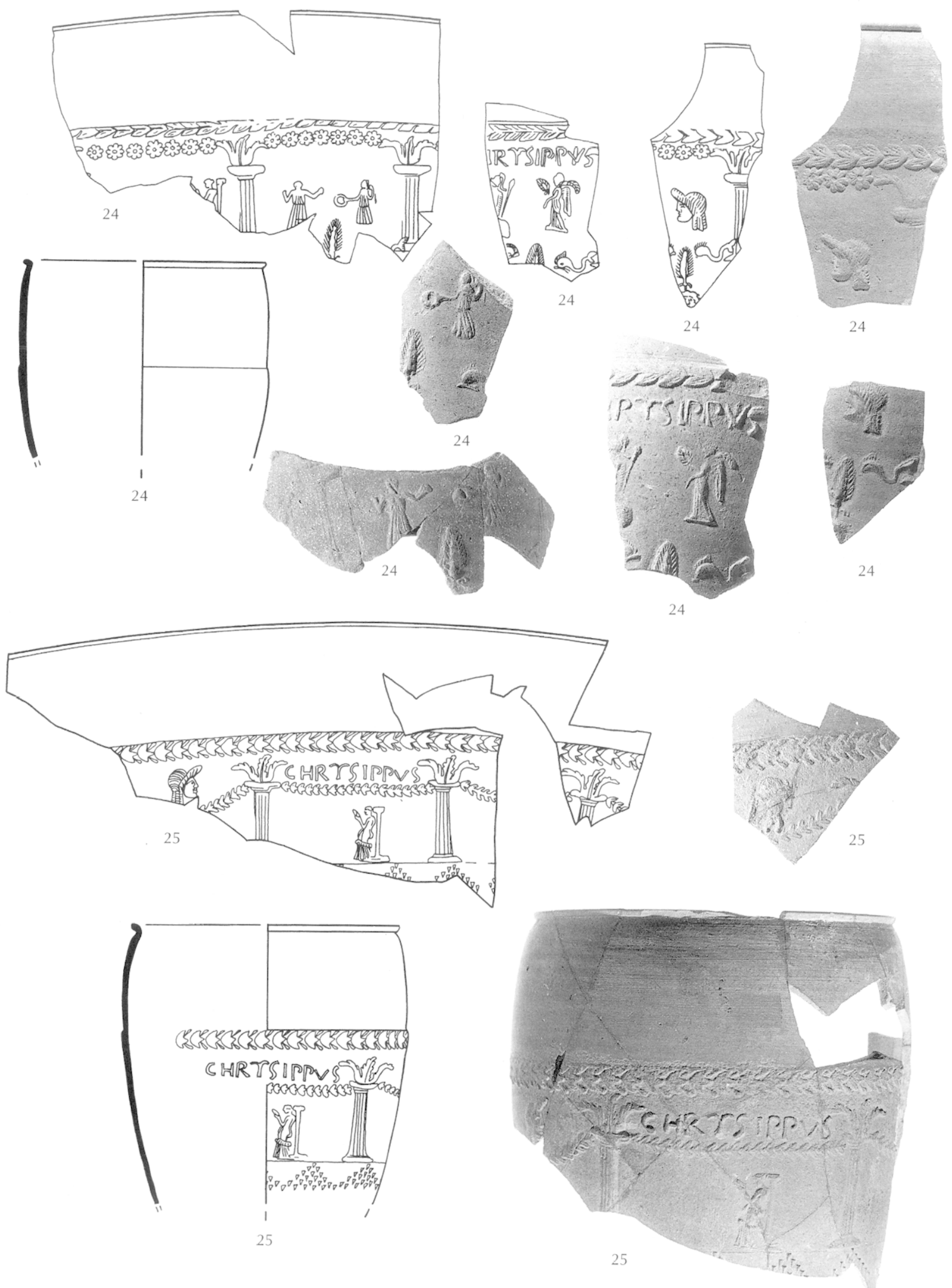

Pl. 56. Chrysipprs, décors 24 et 25 (échelles dessins : 2/3; photos : 1/1). 

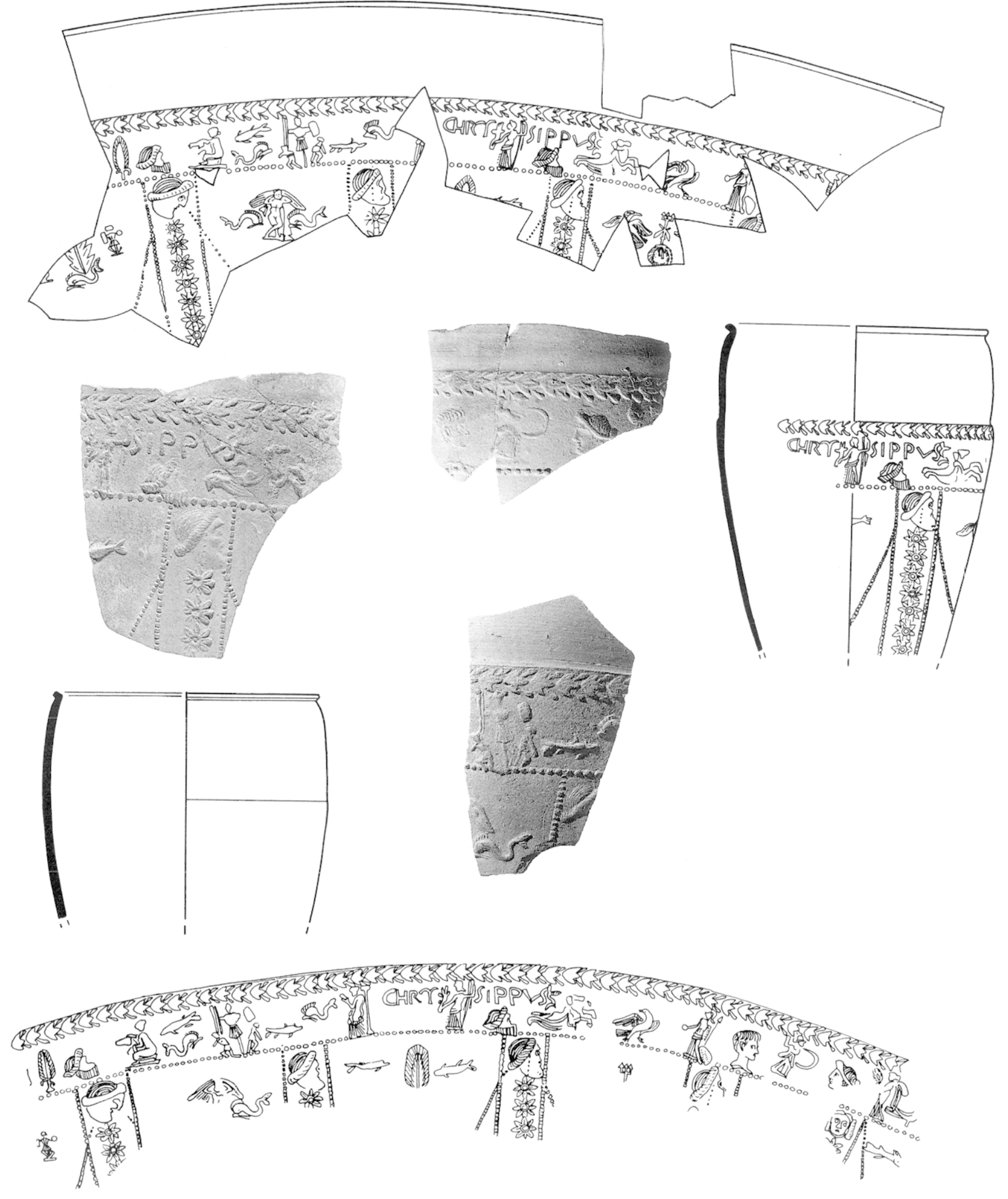

Pl. 57. Chrysipprss, décor 26 (échelles dessins : 2/3; photos : 1/1). 

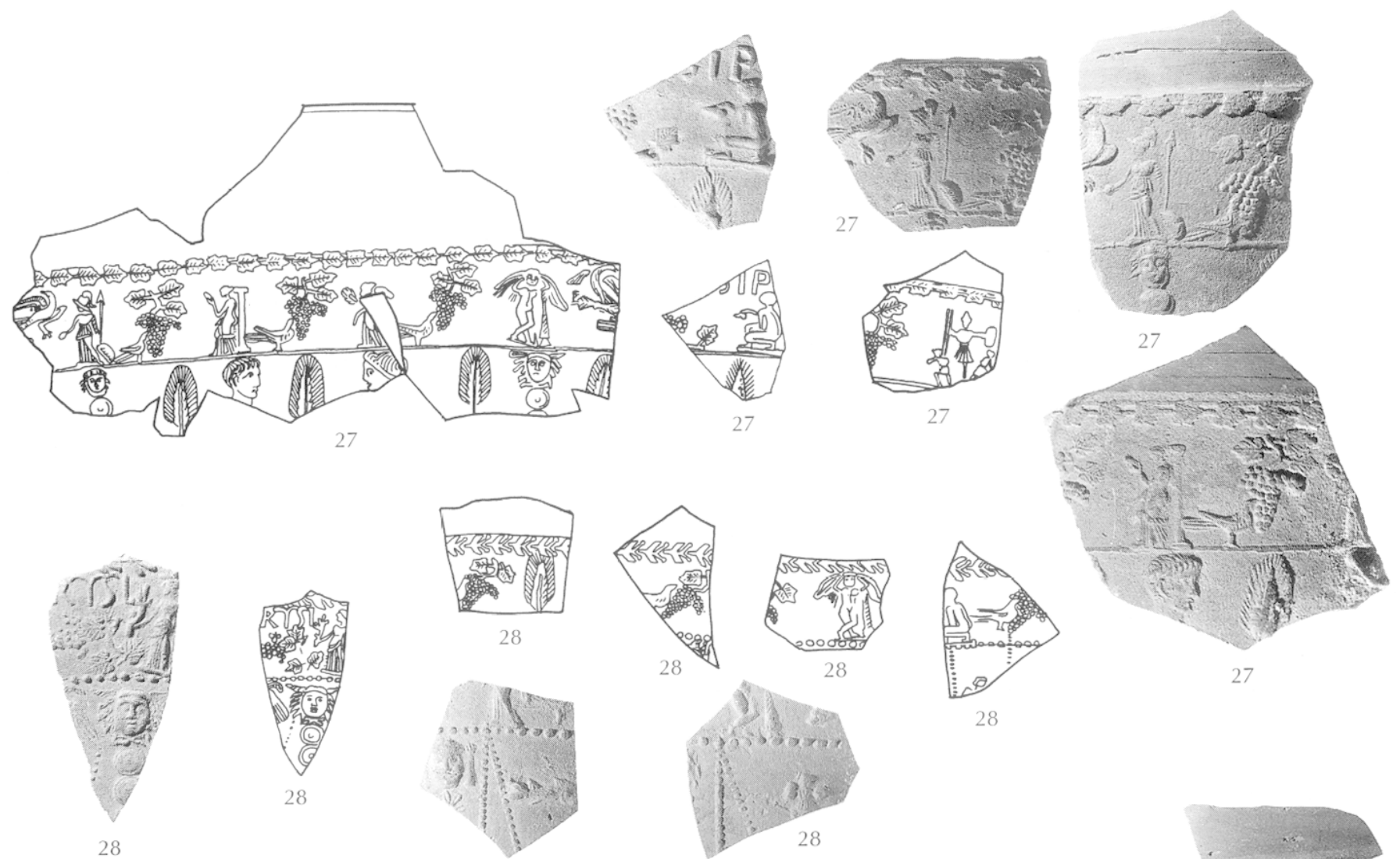

28

28
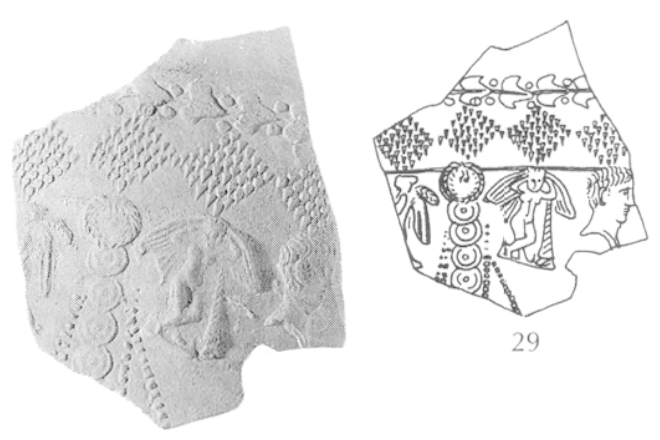

29

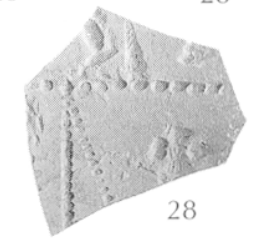

28
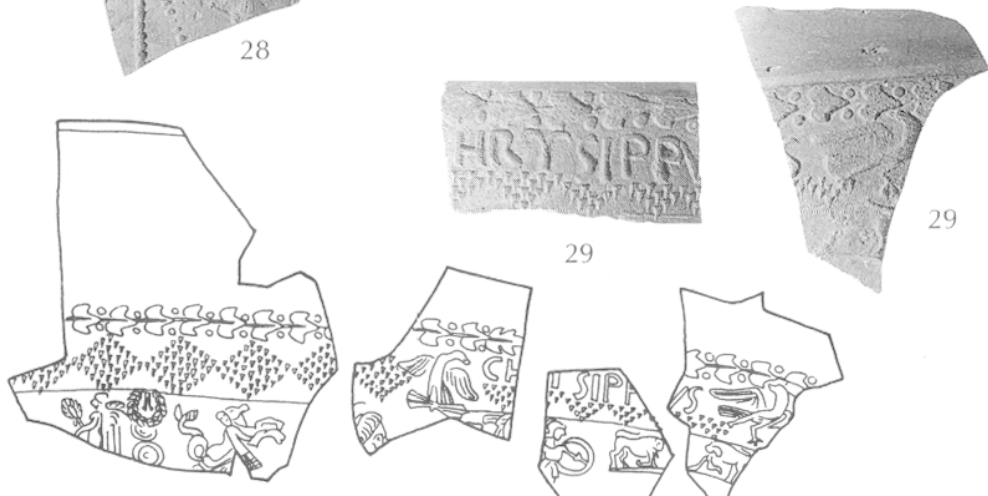

29
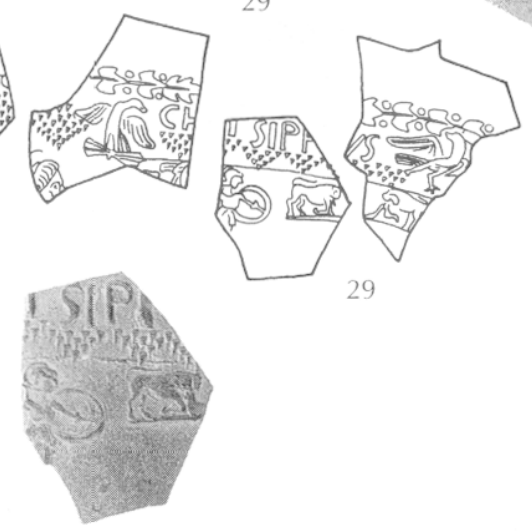

29

29
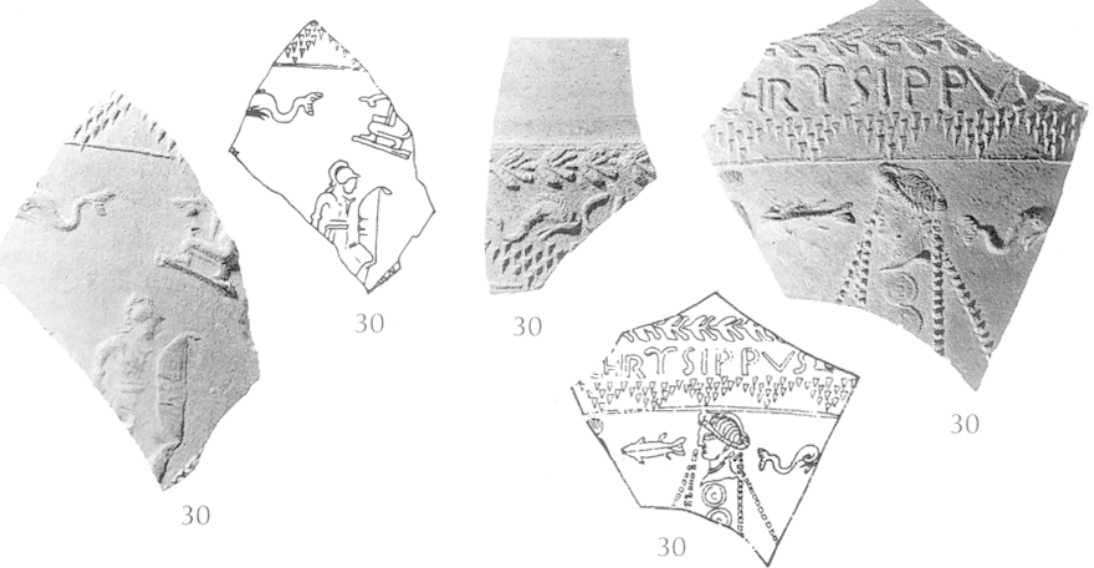

29

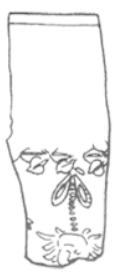

30

Pl. 58. Chrysippres, décors 27, 28, 29, 30) et 31 (échelles dessins : 2/3; photos : 1/1). 


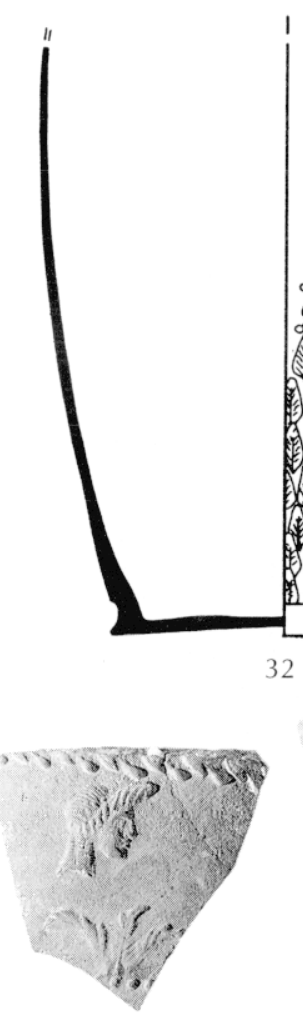

33
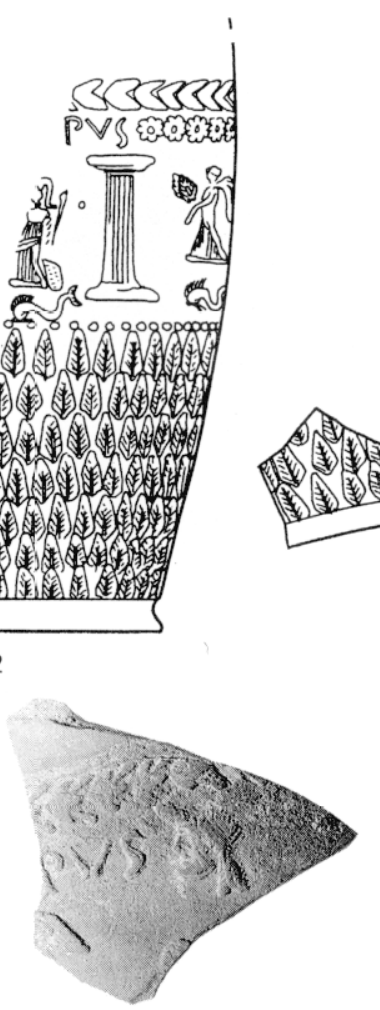

33

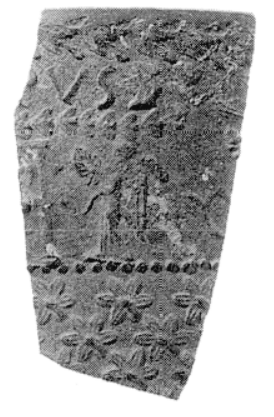

34

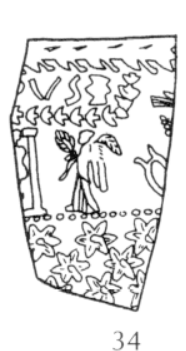

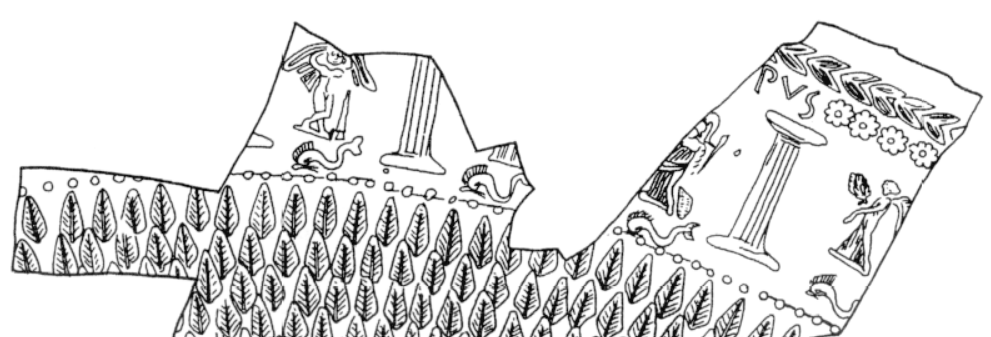

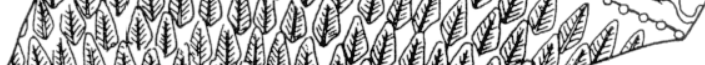

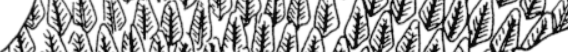

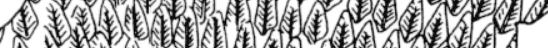
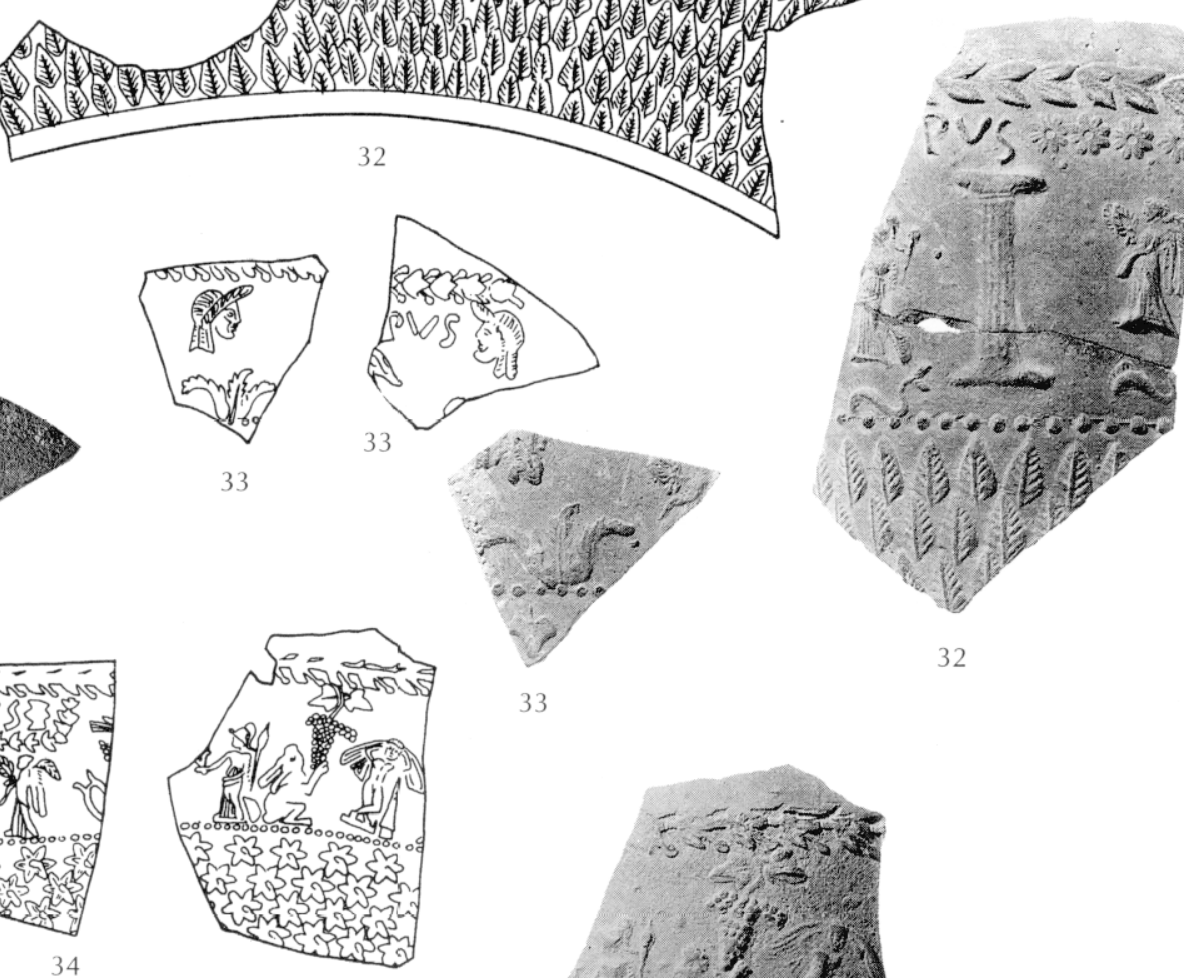

33
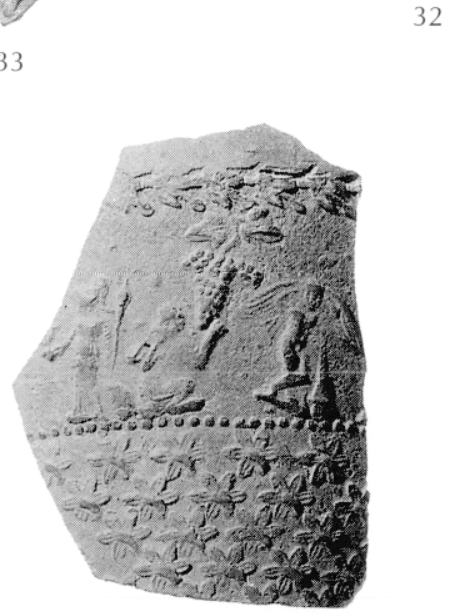

34

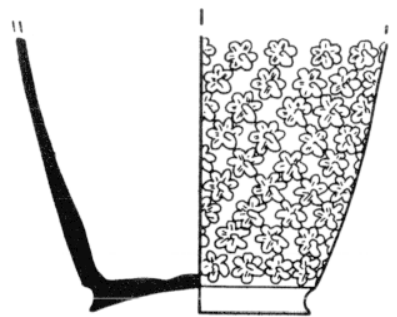

34
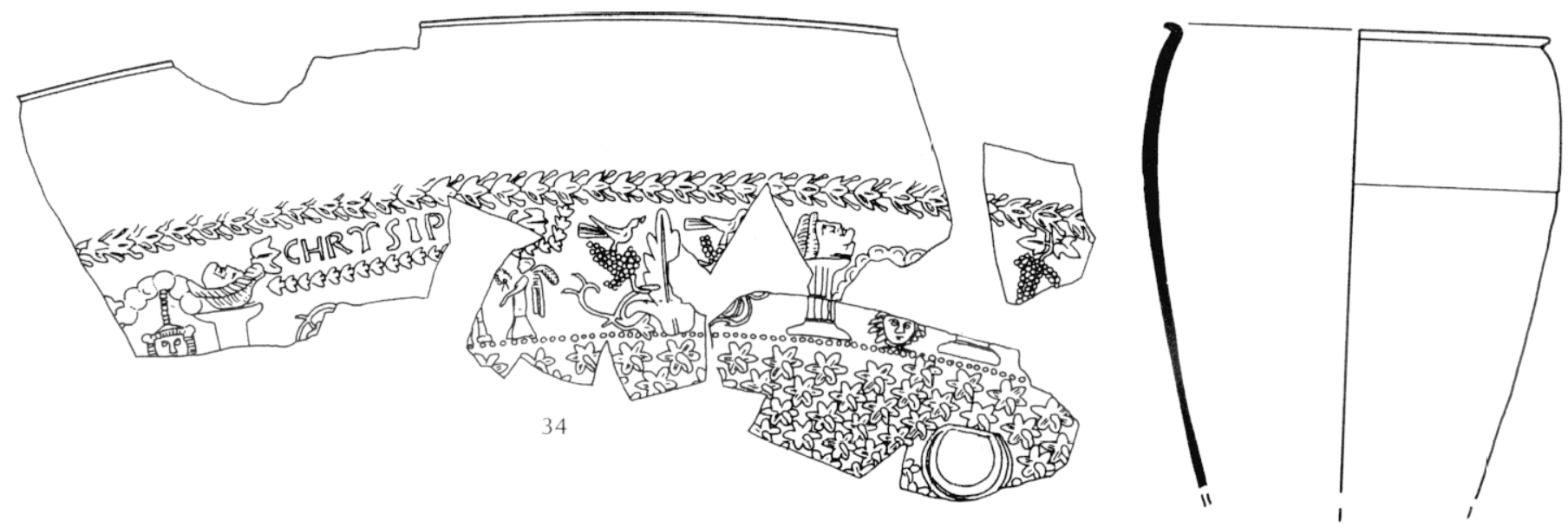

Pl. 59. Chrysipprs, décors 32, 33 et 34 (échelles dessins : 2/3; photos : 1/1). 


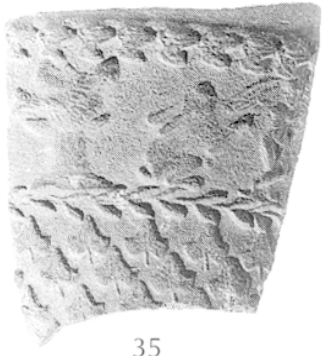

35
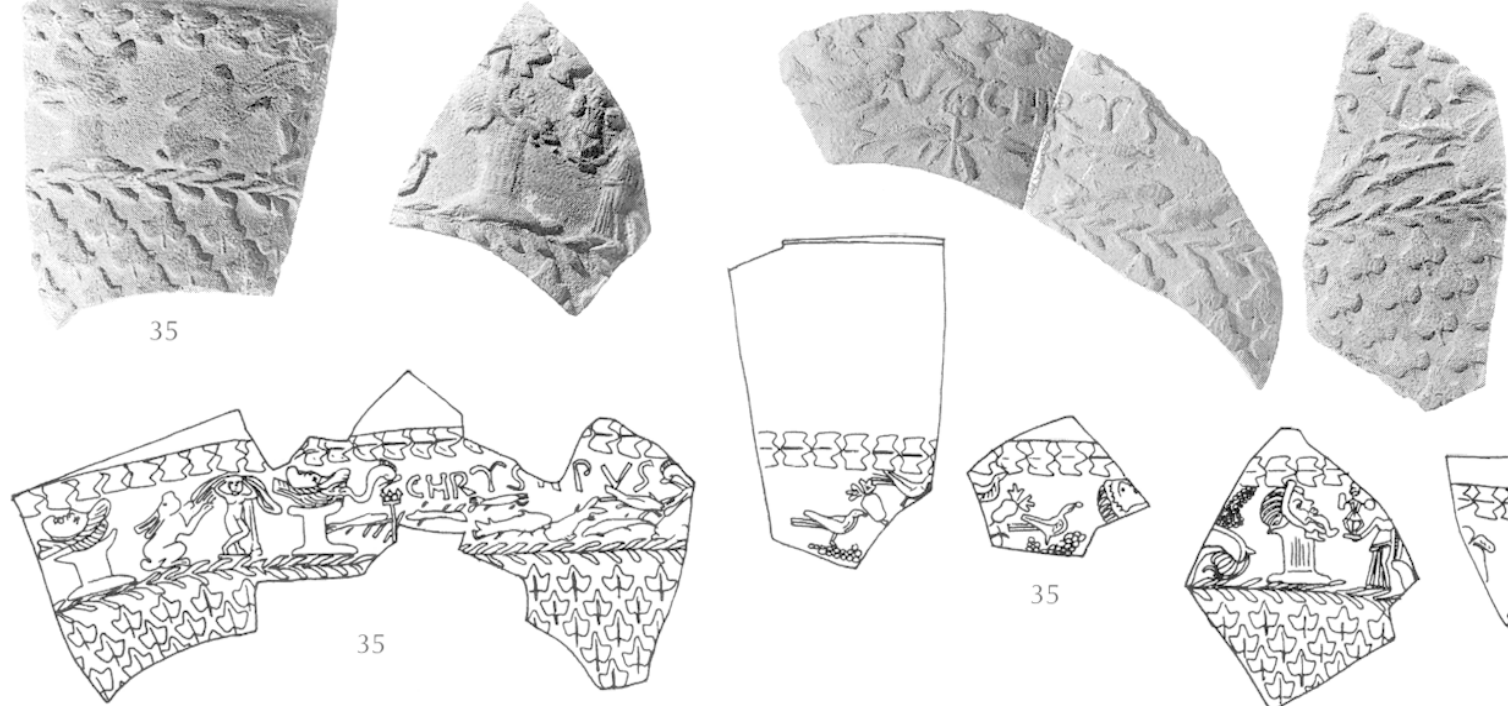

35
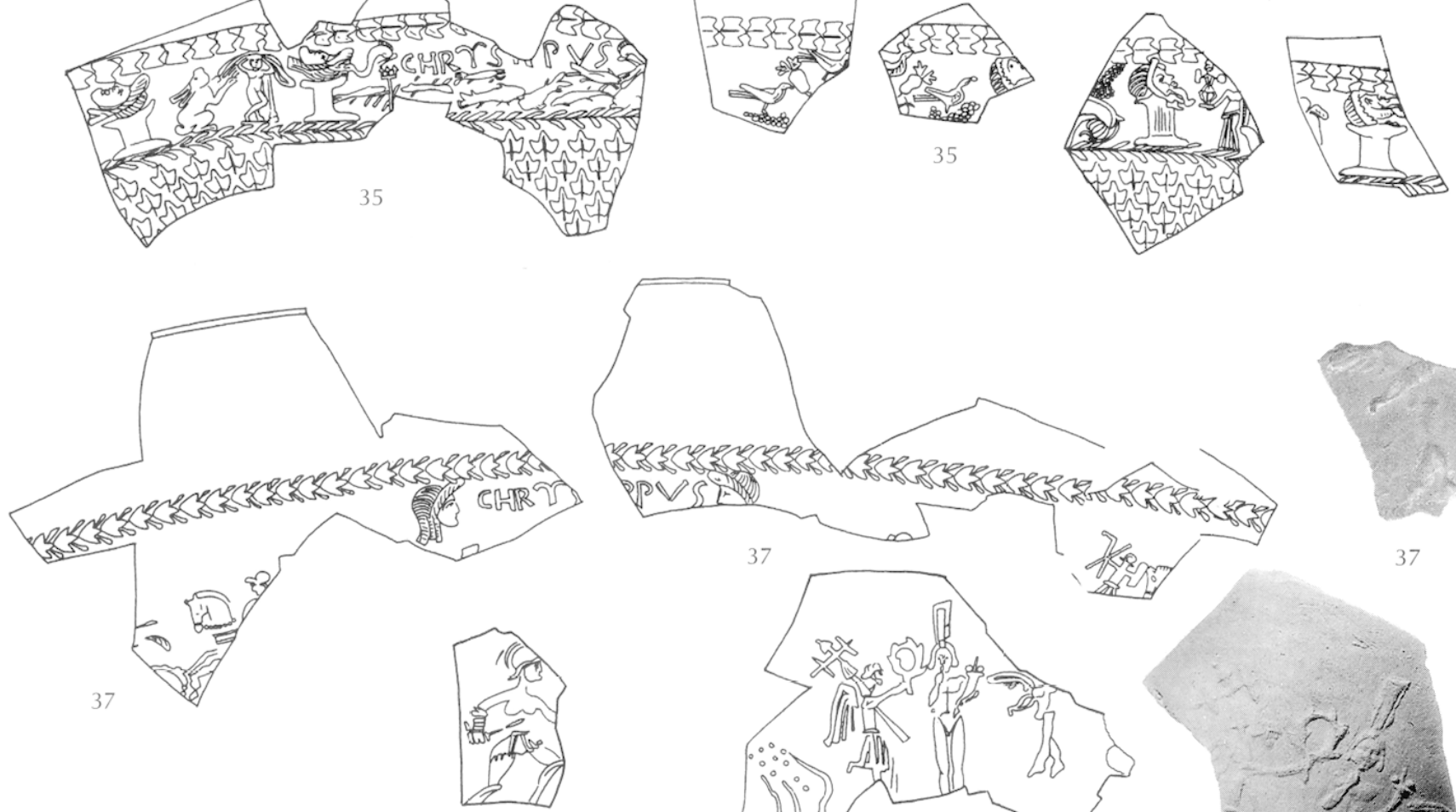

37
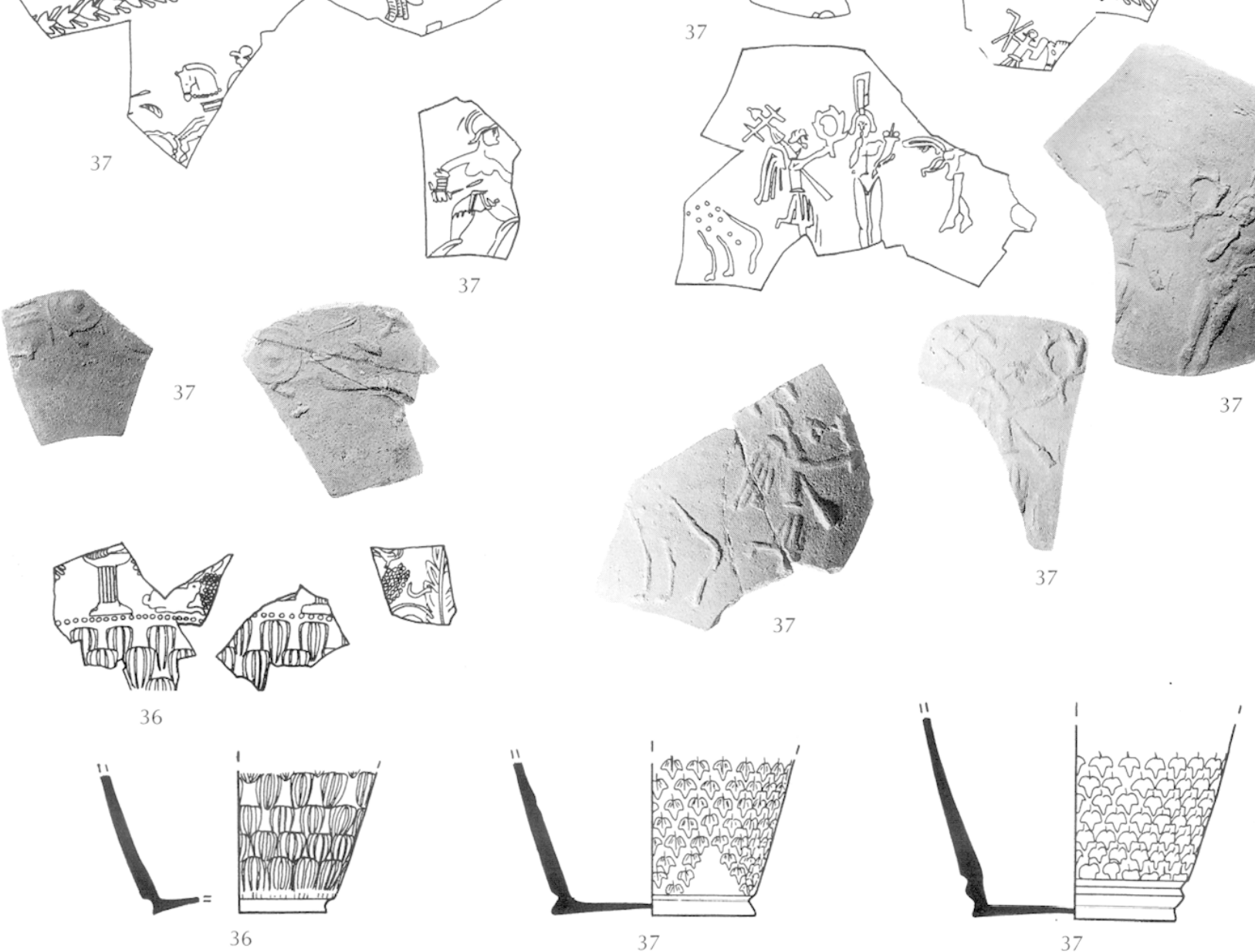

Pl. 60. Chrysippus, décors 35, 36 et 37 (échelles dessins : 2/3; photos : 1/1). 


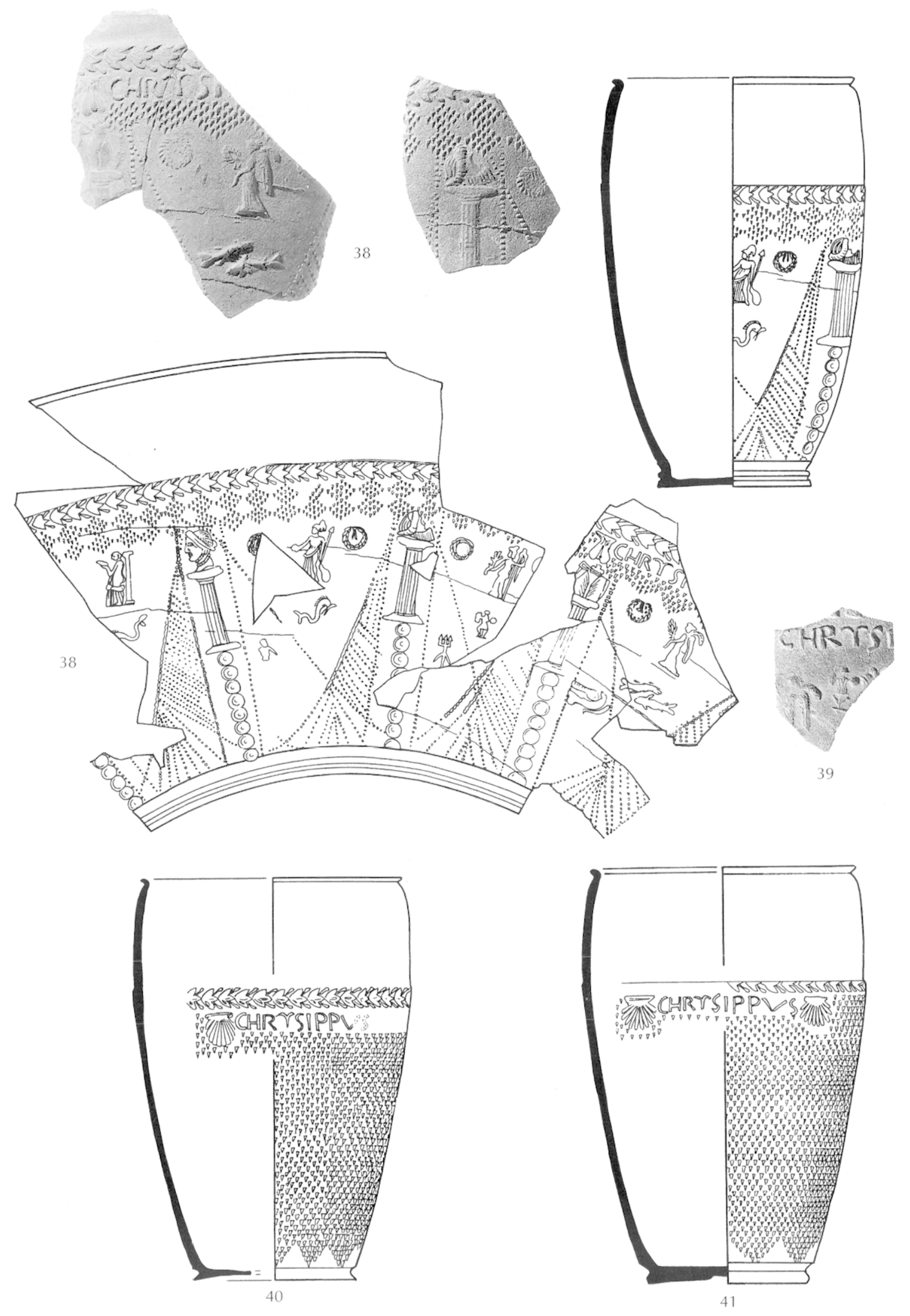

Pl. 61. Chysipprs, décors 38, 39, 40 et 41 (échelles dessins : 2/3; photos : 1/1). 

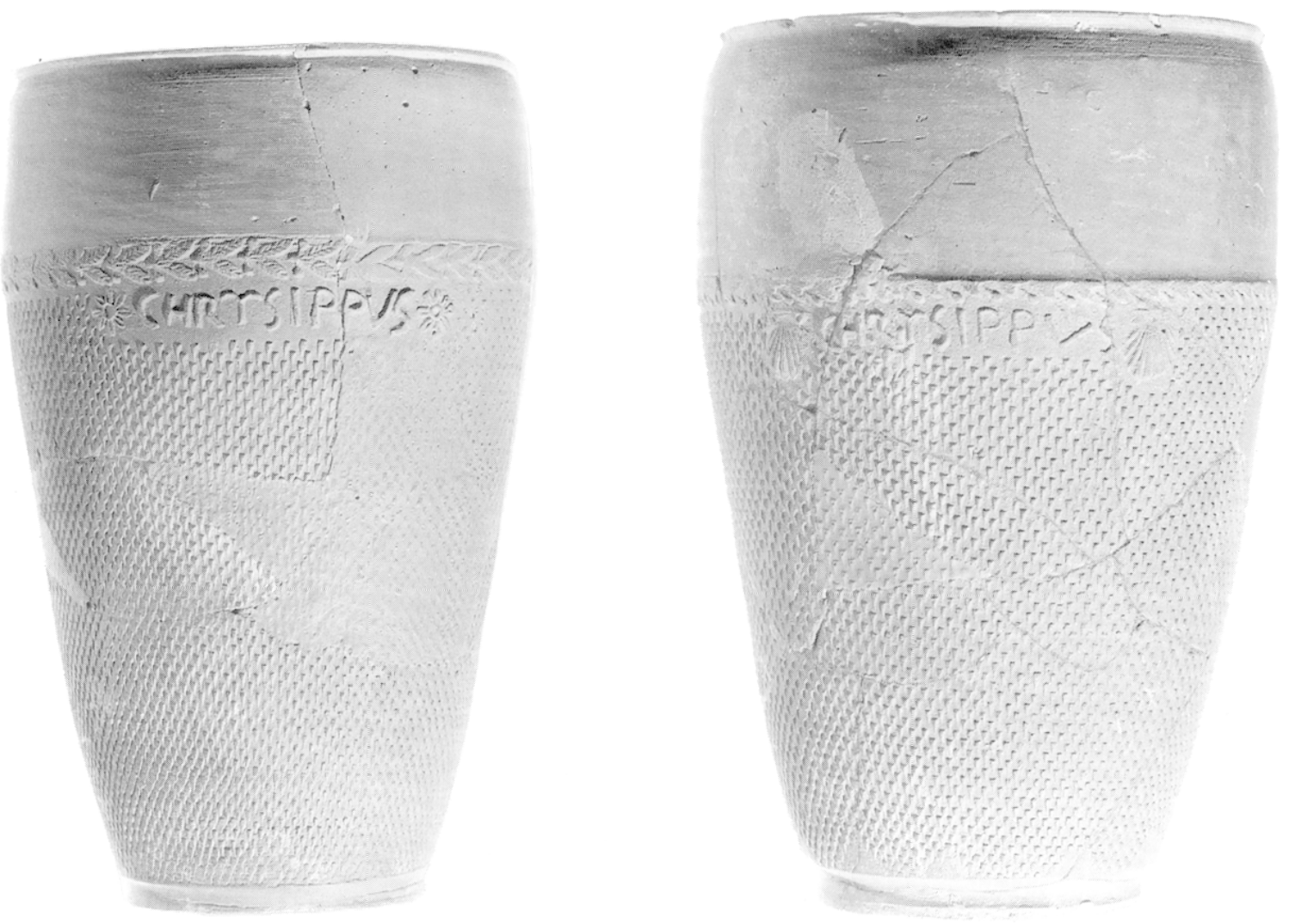

42

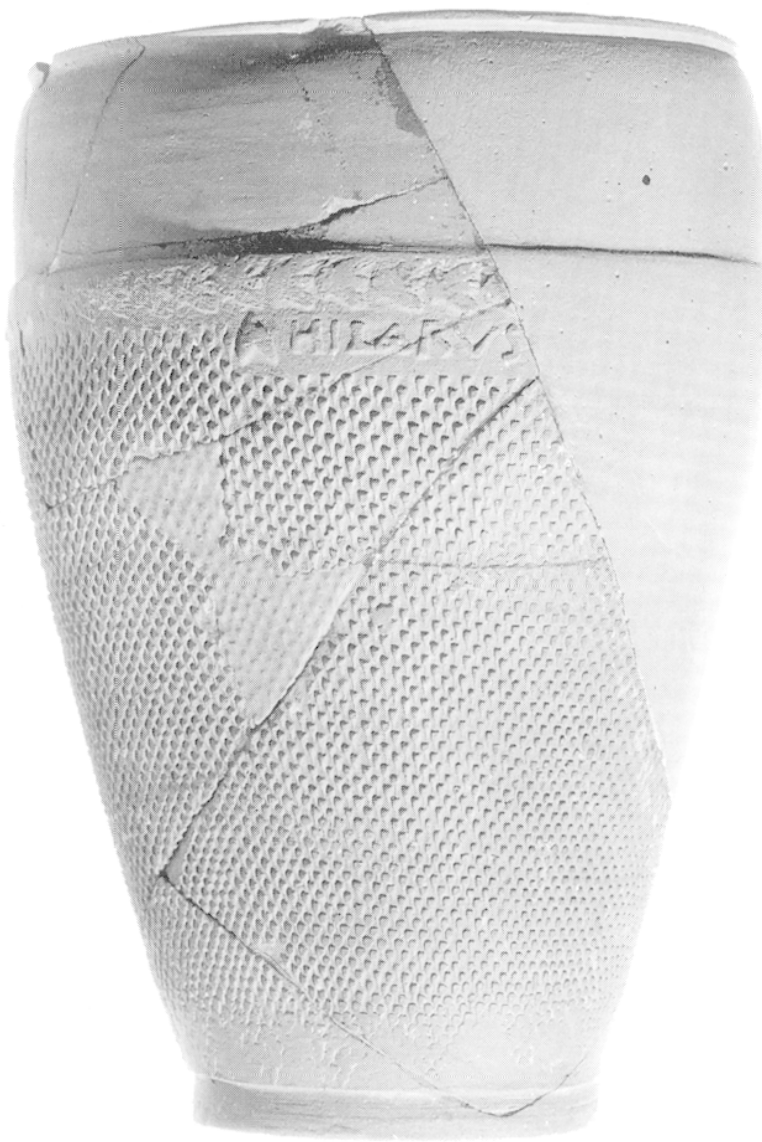

40

18

Pl. 62. Chussippres, décors 40 et 42; Hilares, décor 18 (échelle: $1 / 1$ ). 

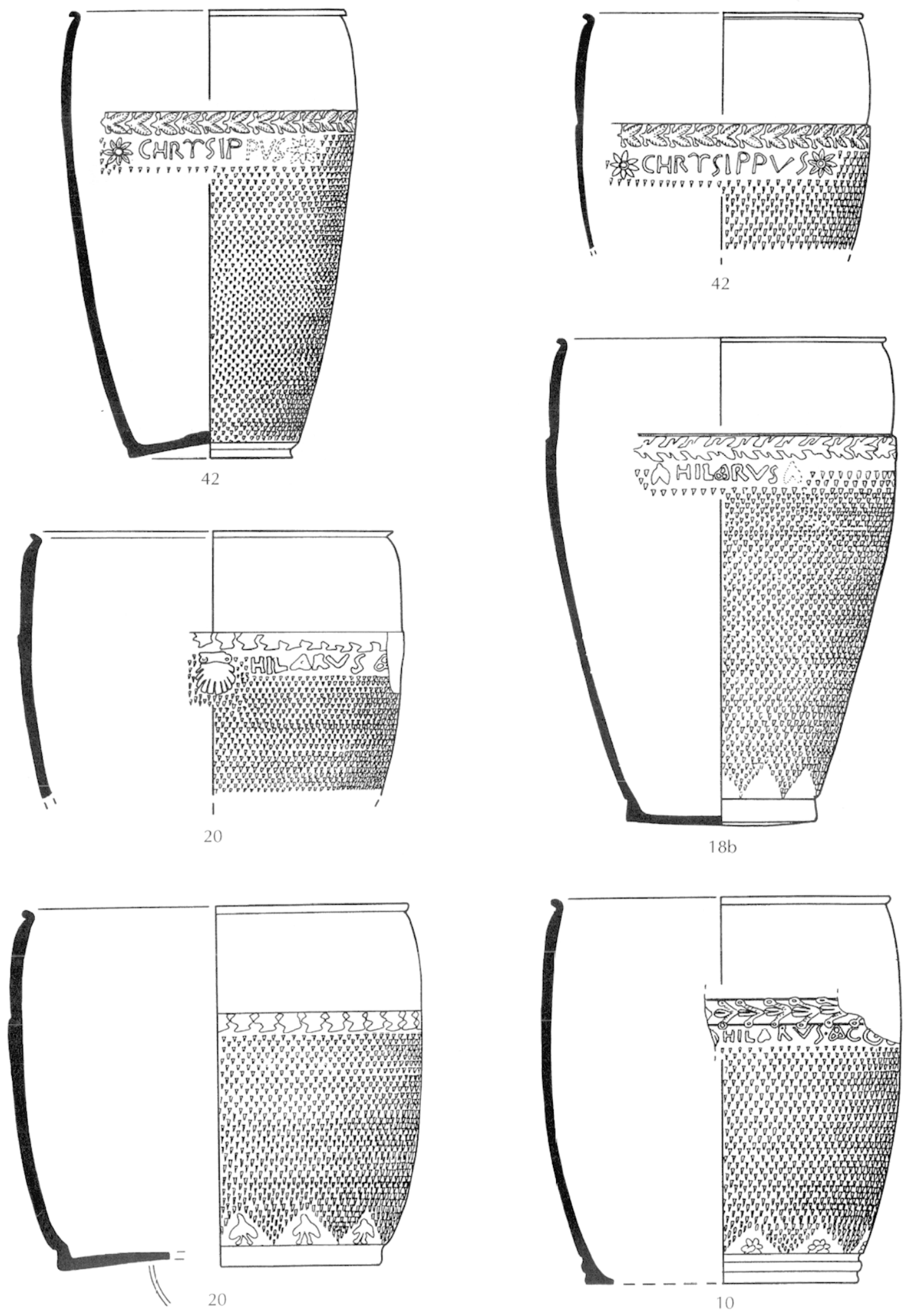

P1. 63. Chrysippus, décor 42 et Hilarus Aco, décors 10. 186 et 20 (échelle: 2/3). 


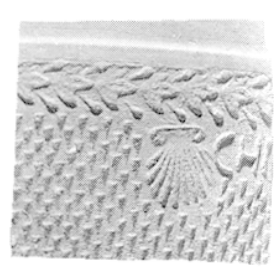

$40 \mathrm{a}$

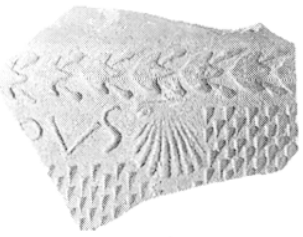

40

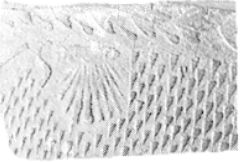

$41 \mathrm{~b}$

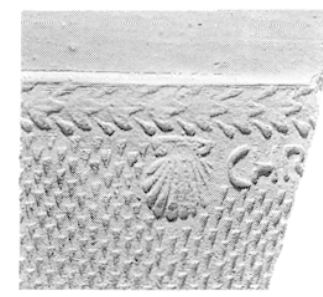

$40 \mathrm{~b}$

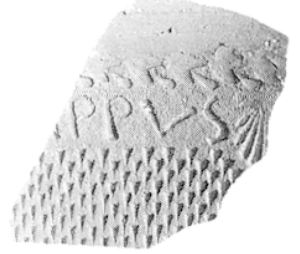

40

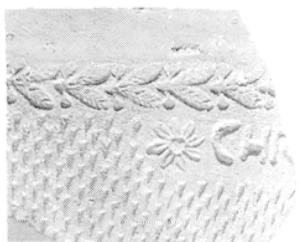

$42 \mathrm{a}$

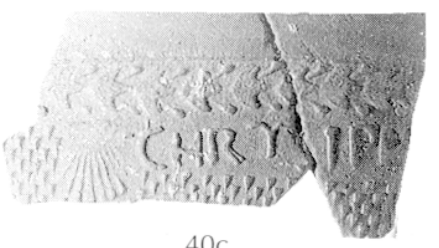

$40 c$

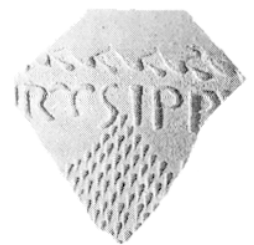

$41 \mathrm{a}$

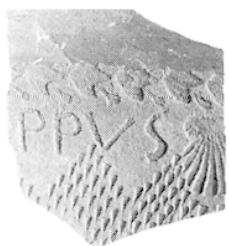

$41 \mathrm{a}$

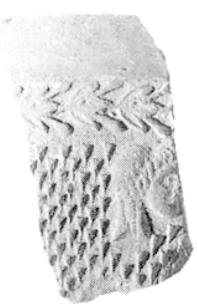

44

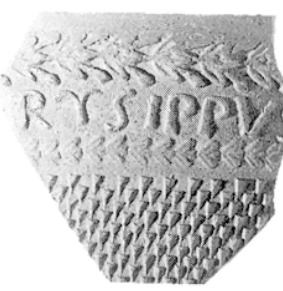

44

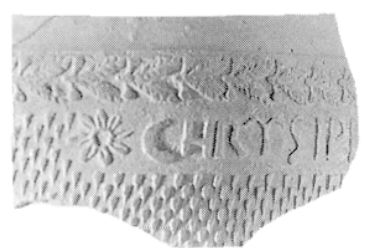

$42 b$

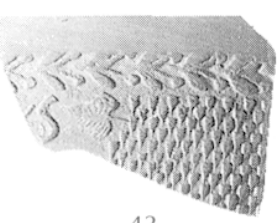

43

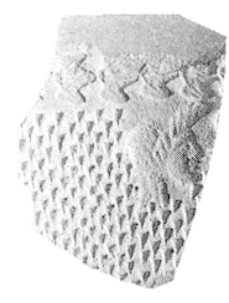

45

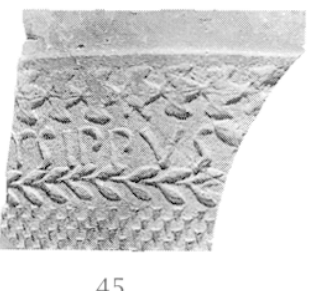

45

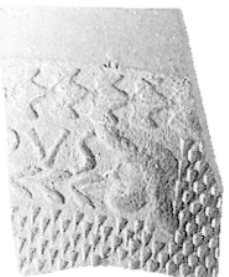

45

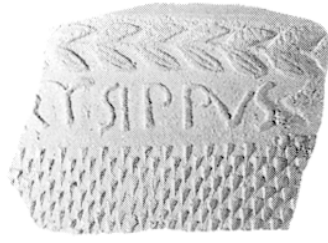

46

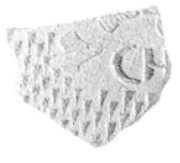

47

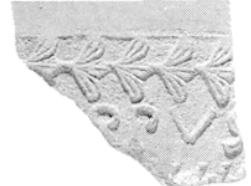

48

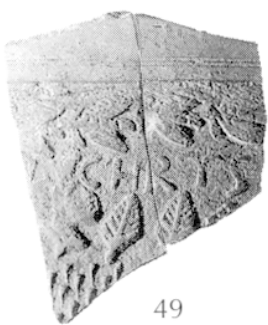

P1. 64. Décors de Chrysippus (échelle : 1/1). 

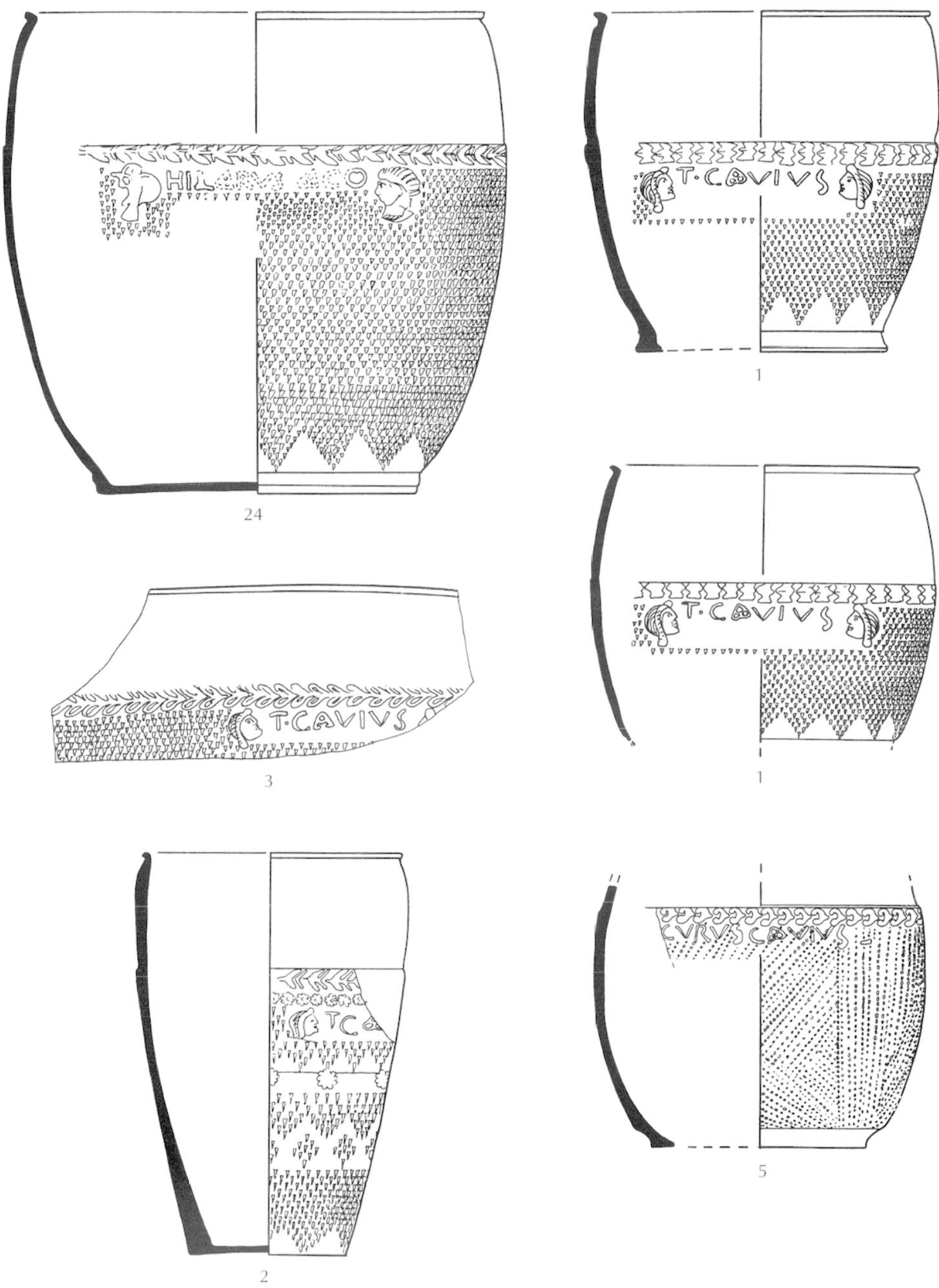

Pl. 65. Hilarus Aco, décor 24 et T. Cavius, décors 1, 2, 3 et 5 (échelle: 2/3). 

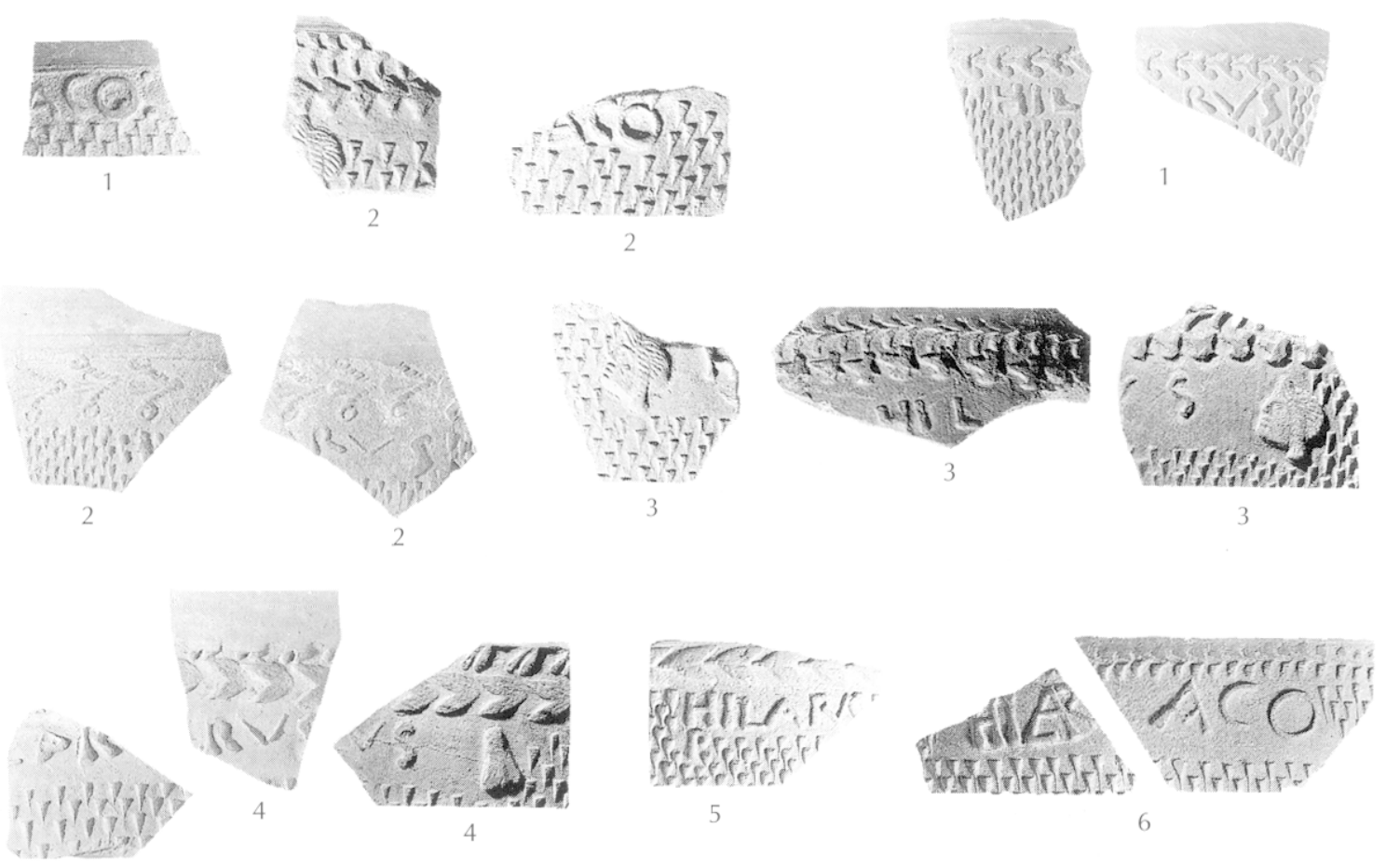

4

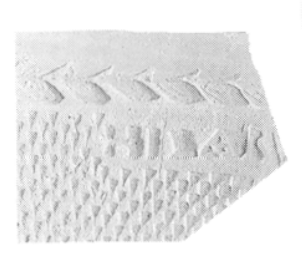

$7 a$

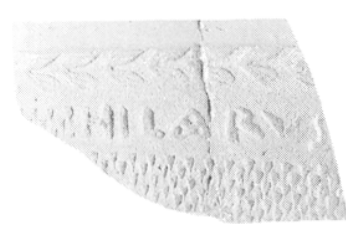

$7 d$
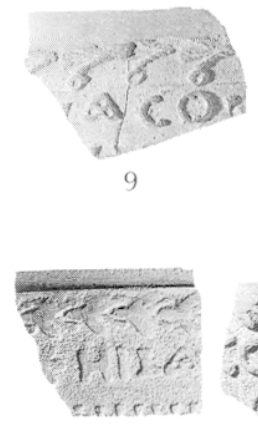

12

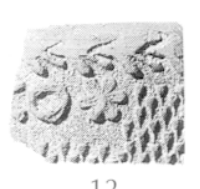

12
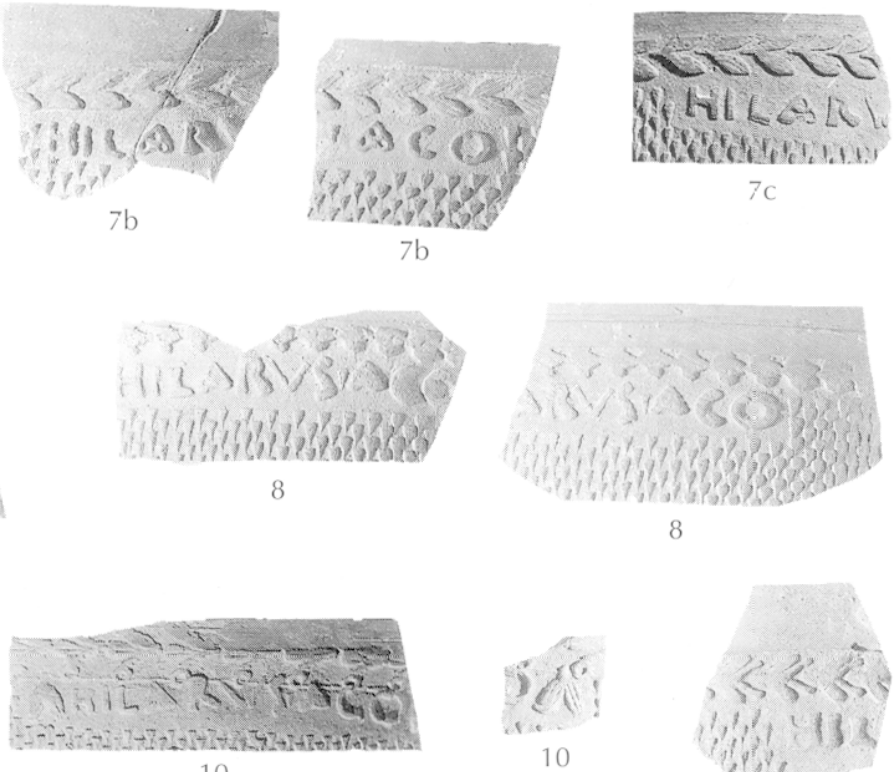

$7 \mathrm{C}$

$7 \mathrm{~b}$

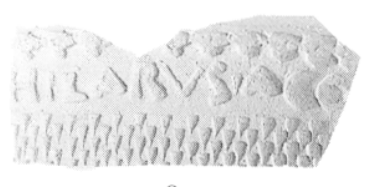

8

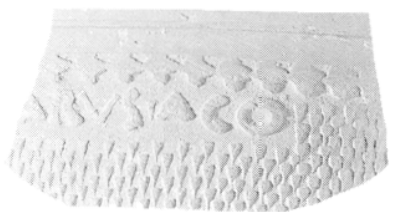

8

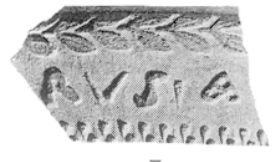

$7 \mathrm{C}$

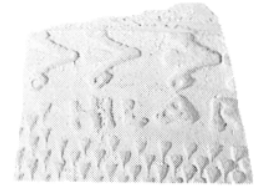

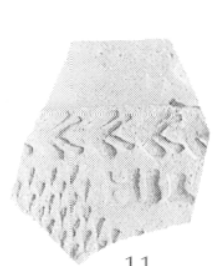

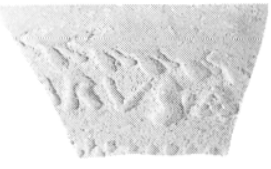

11

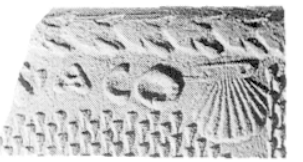

13

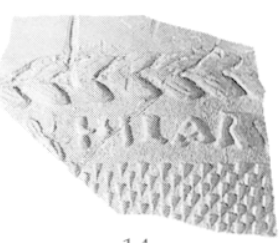

14

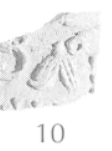

11

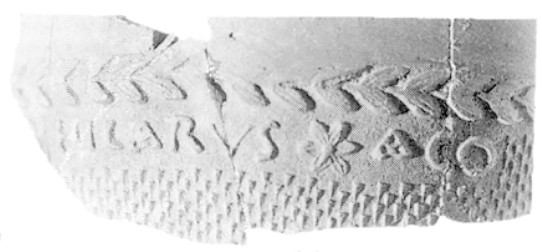

14

P1. 66. Décors d'Aco et d'Hilarvs Aco (échelle : 1/1). 


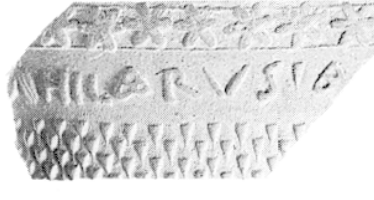

15

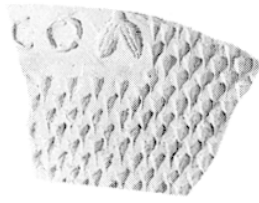

15

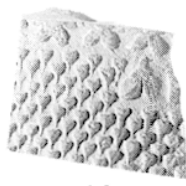

16

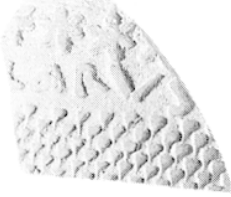

$16 \mathrm{a}$

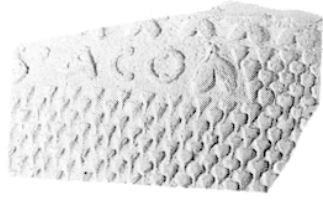

$16 \mathrm{a}$

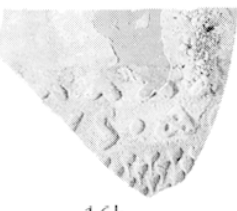

$16 b$

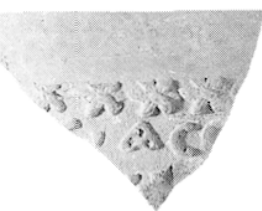

17

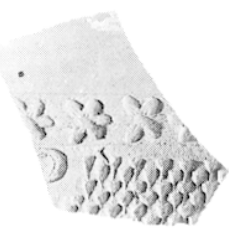

17

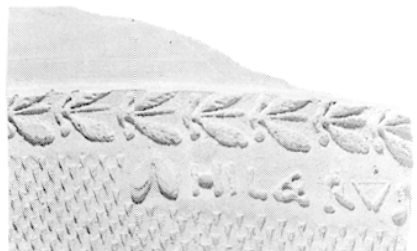

18

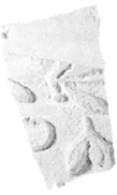

18

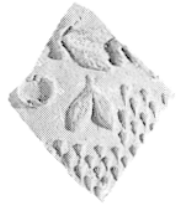

18

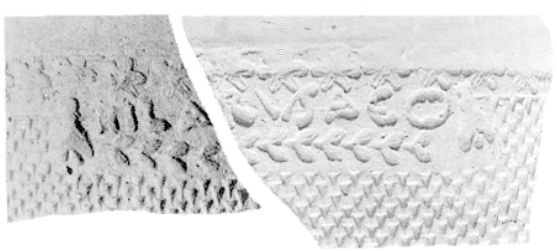

19

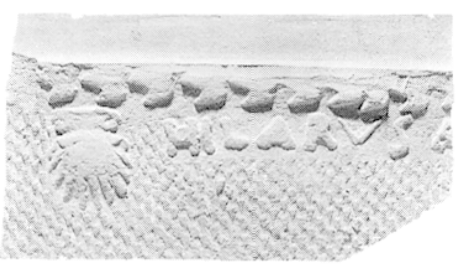

20

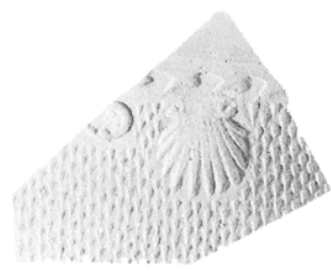

20

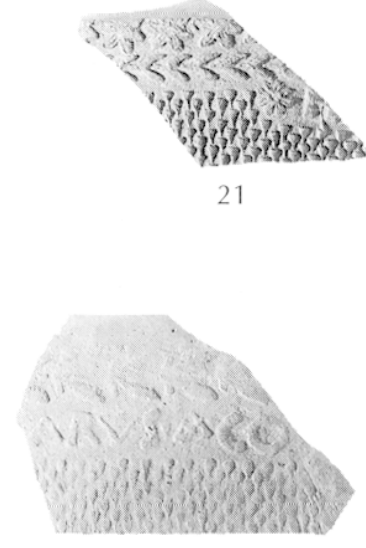

24

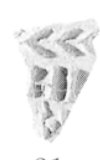

21

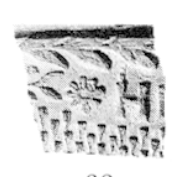

22

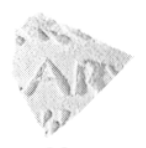

23

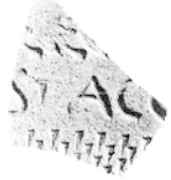

23

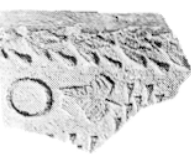

23
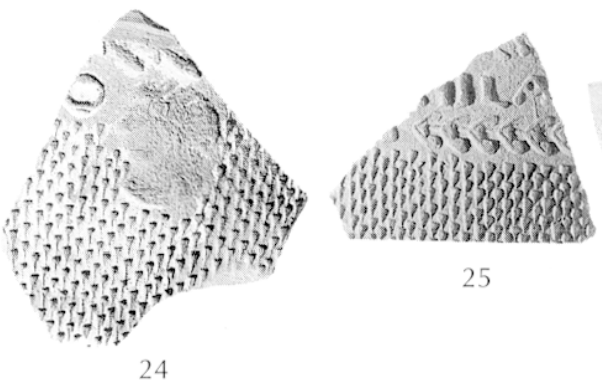

25

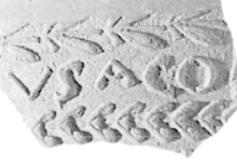

25

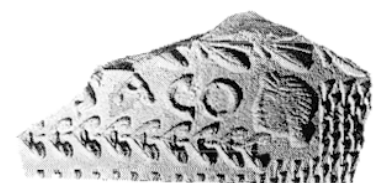

25

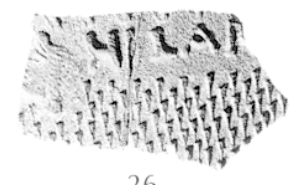

26

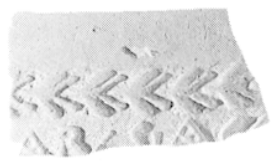

27

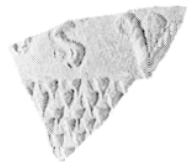

28

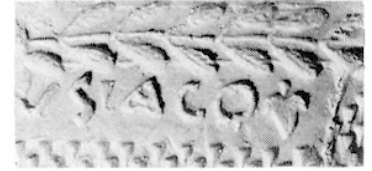

29

Pl. 67. Décors d'Hilarus Aco (échelle: 1/1). 

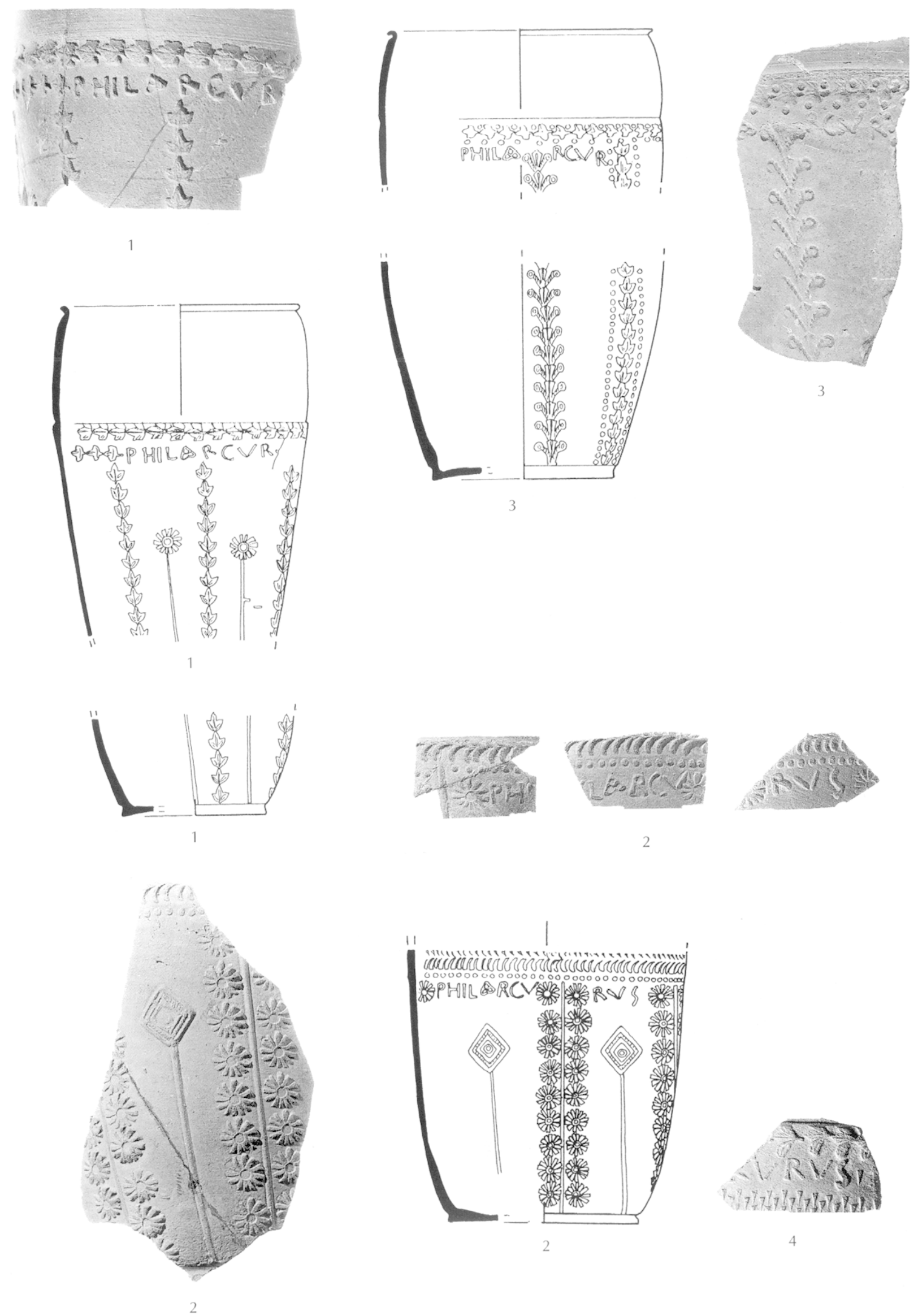

Pl. 68. Décors de Philarrorus (échelles dessins : 2/3; photos: 1/1). 

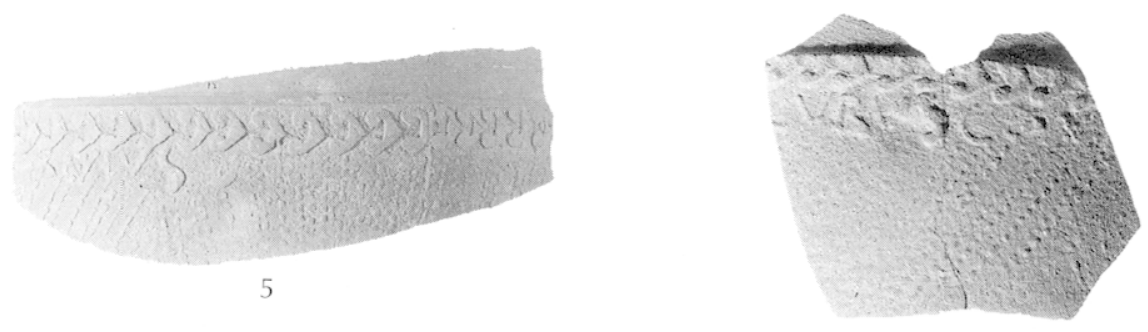

5
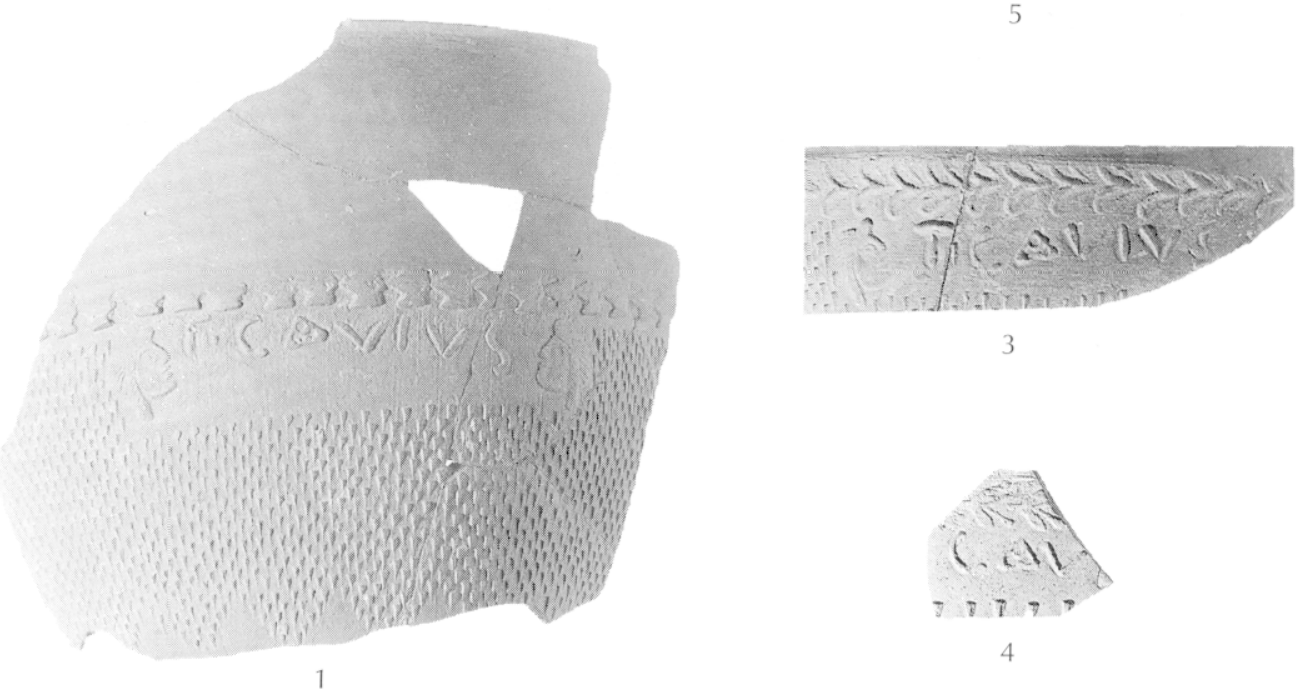

3
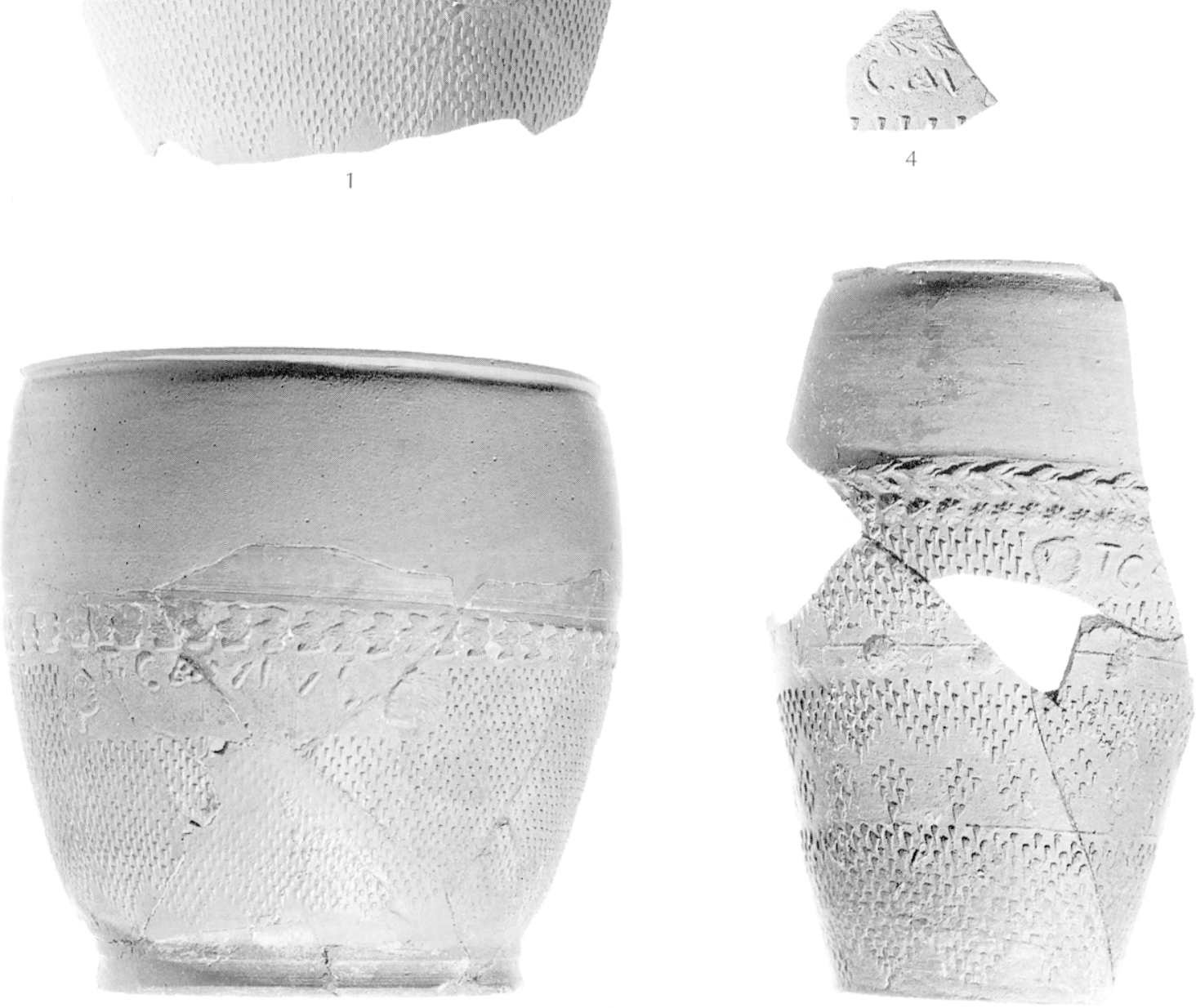

1

Pl. 69. Décors de T. Cavius (échelle : 1/1). 

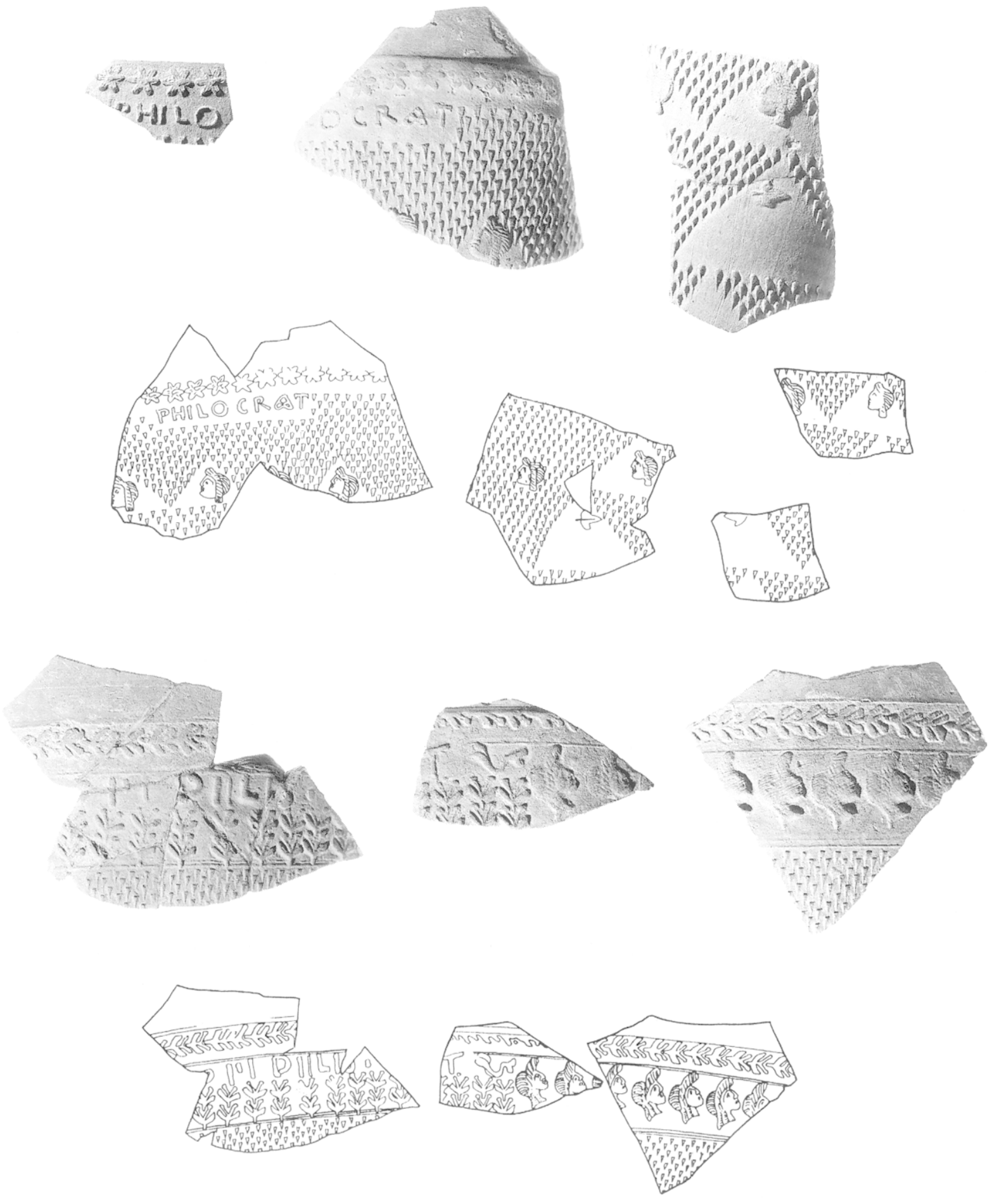

Pl. 70. Décors de Philocrates et de Fïdelis (échelles dessins: $2 / 3$; photos: $1 / 1$ ). 

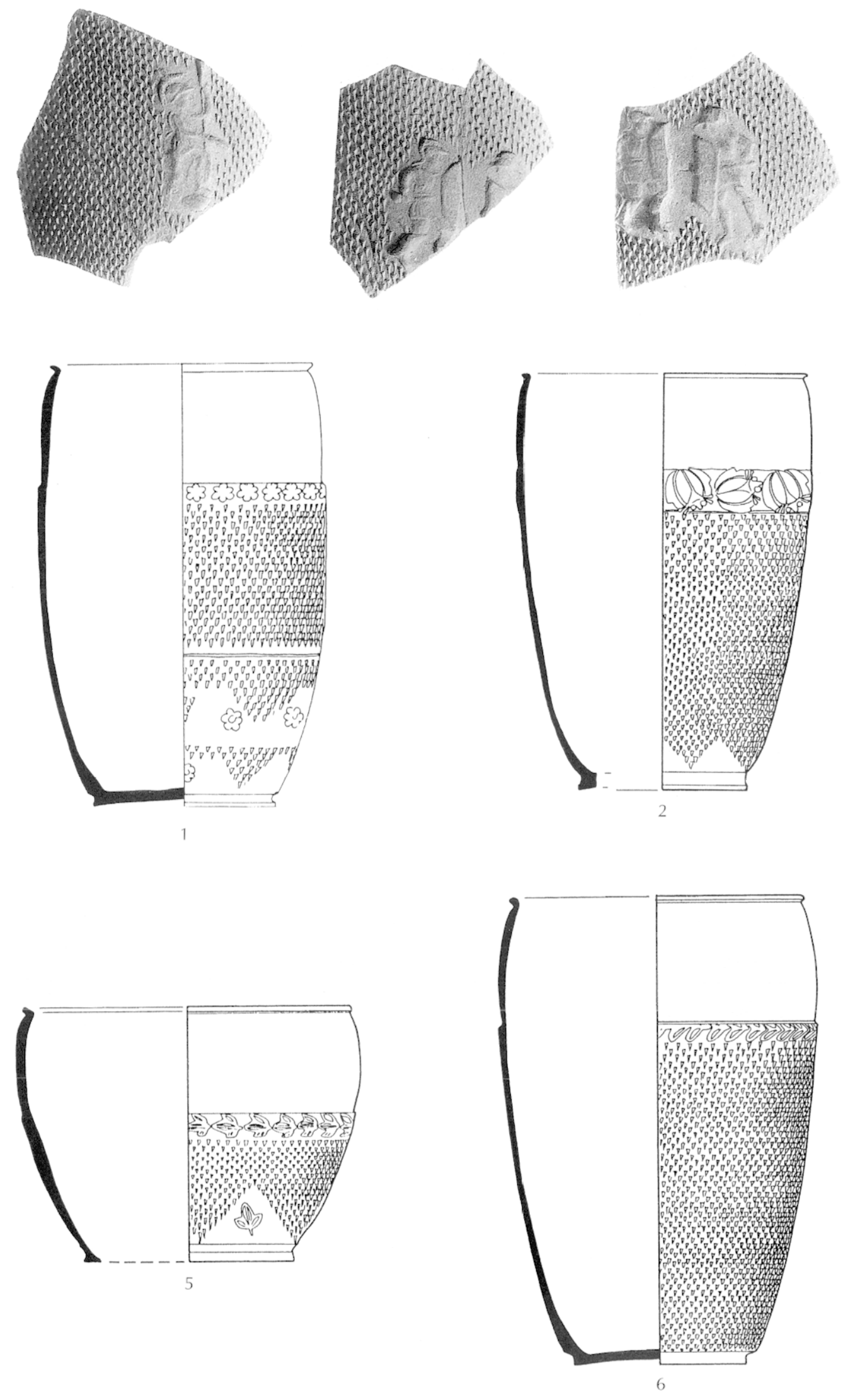

P1. 71. Décors non signés (échelles dessins : 2/3; photos: 1/1). 

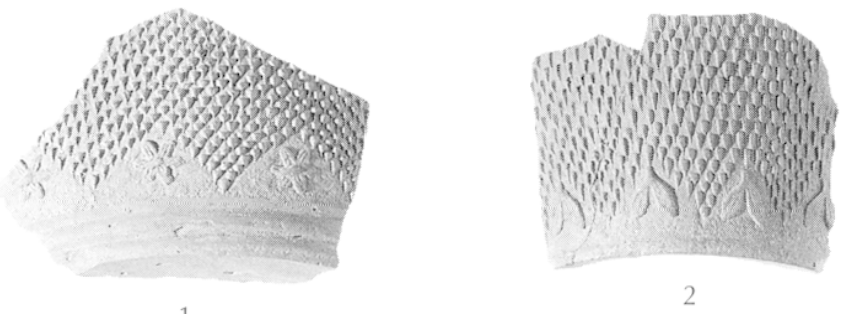

2

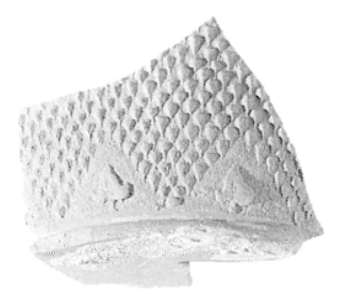

4

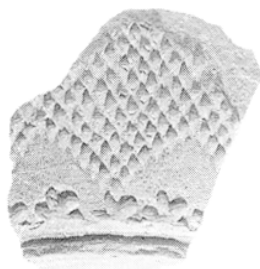

6

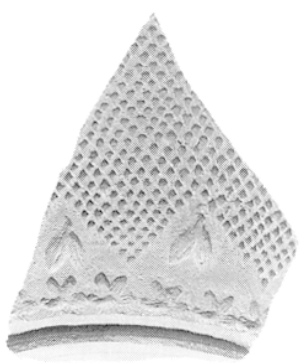

8

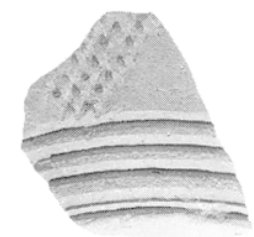

11

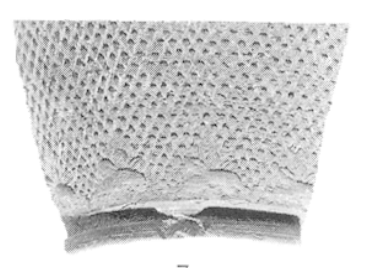

7

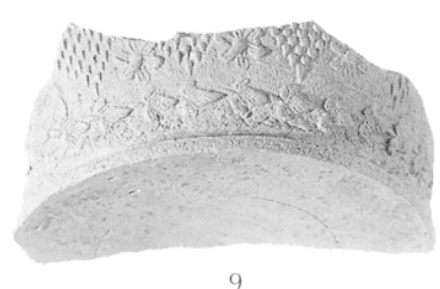

9

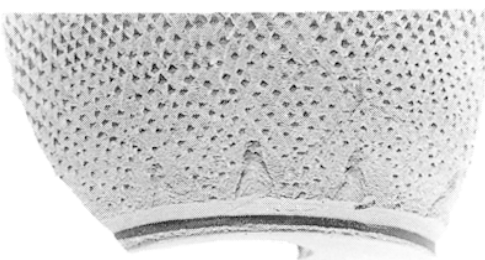

3
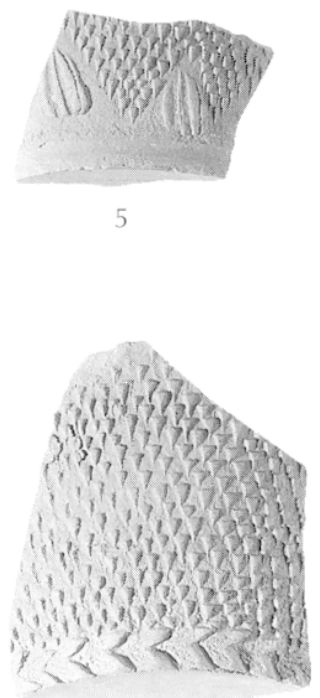

10

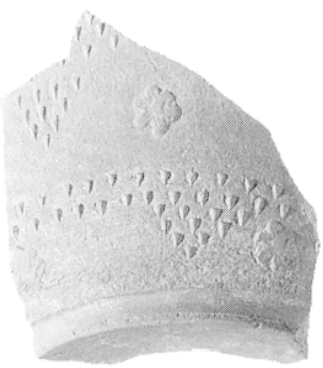

12

P1. 72. Fonds de gobelets (décors indéterminés) (échelle : 1/1). 

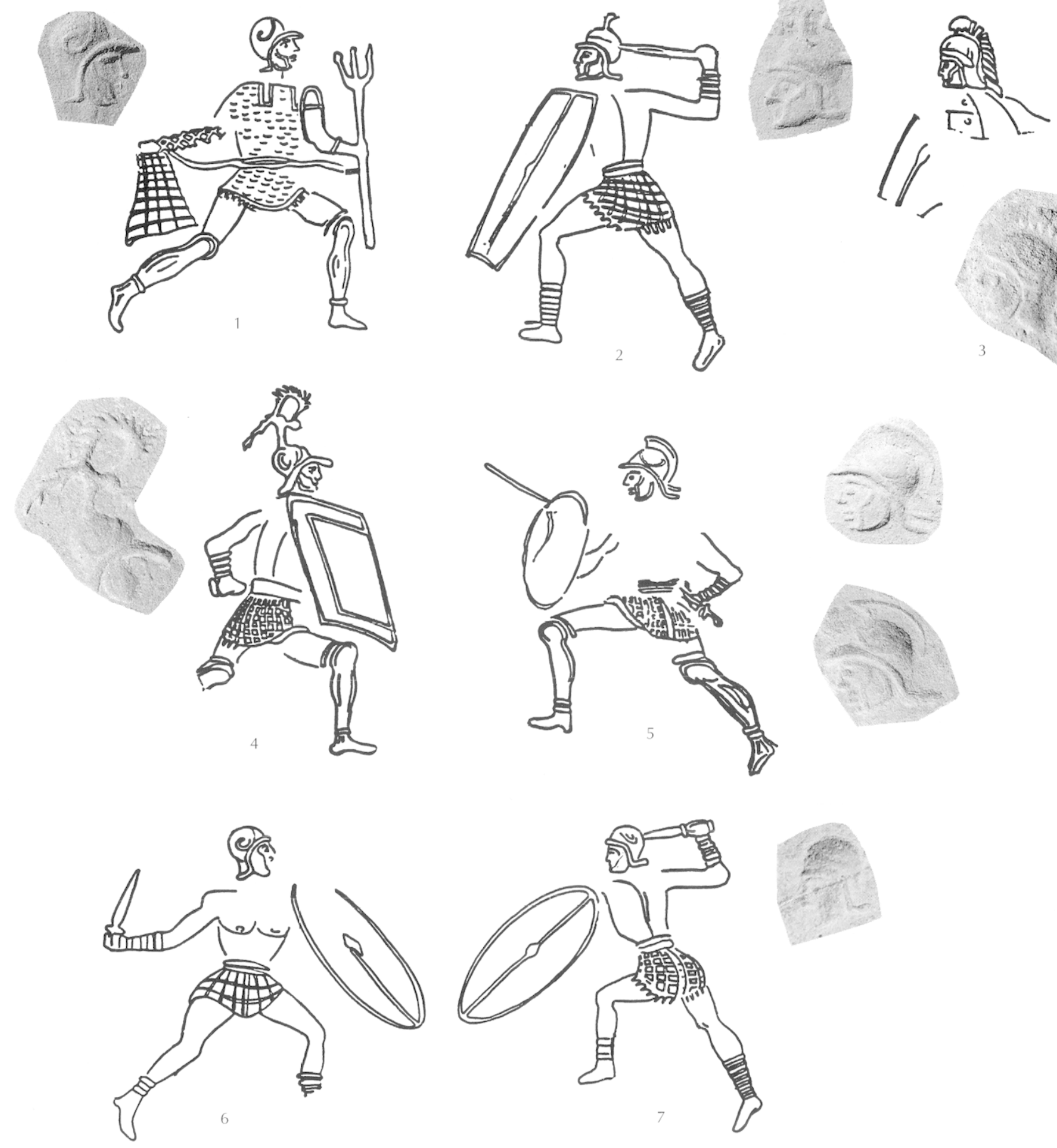

P1. 73. Poincons de (hrysippres, gladiateurs (échelles dessins : $1 / 1$; photos : 2/1). 

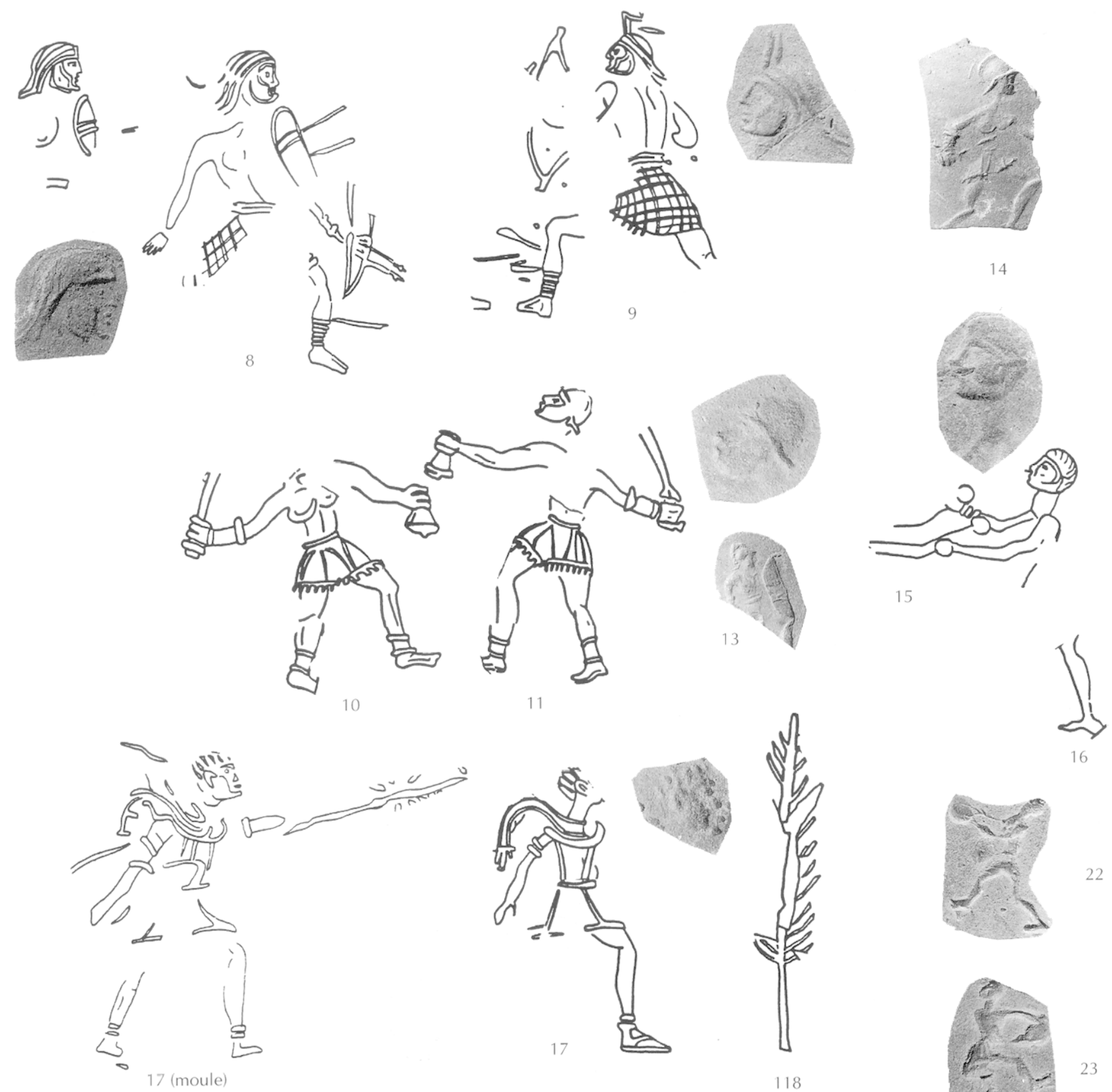

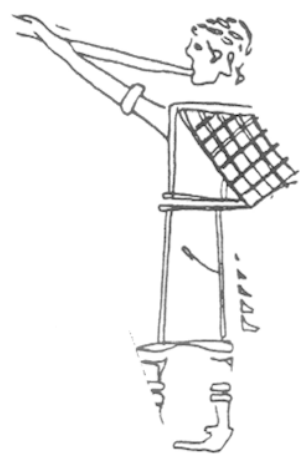

18

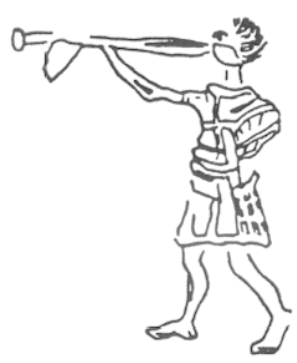

19

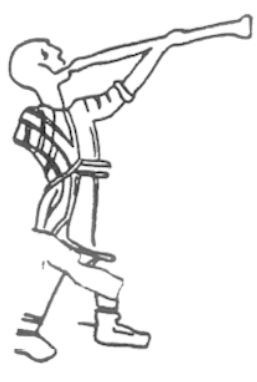

20

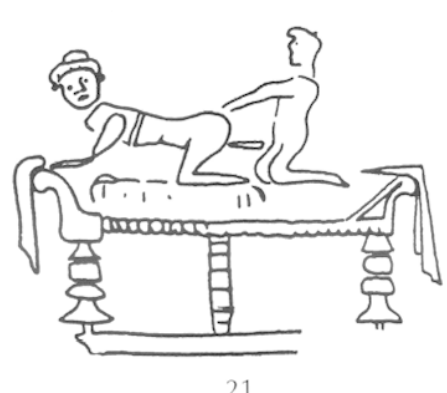

P1. 74. Poinsons de Chysippris, gladiateurs, autres personnages et motif végétal (échelles dessins : $1 / 1$; photos : 2/1 sauj 14, 22, 23, échelle : 1/1). 


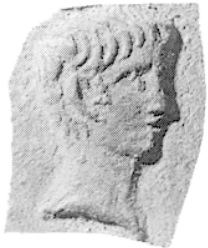

26

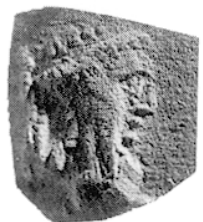

31

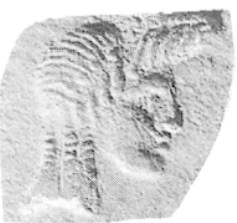

$36 a$

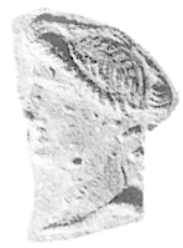

27

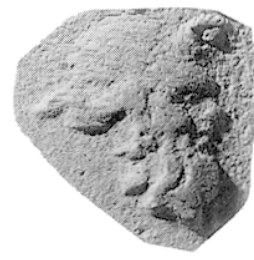

32

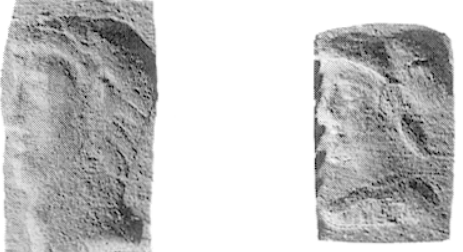

29

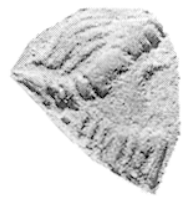

34

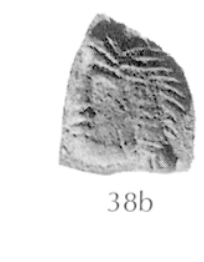

$38 b$
$38 \mathrm{a}$

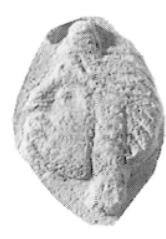

$36 b$

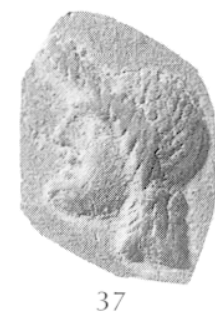

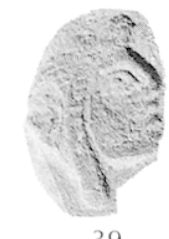

39

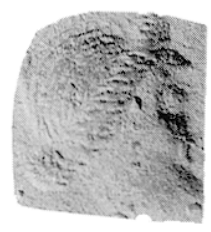

35

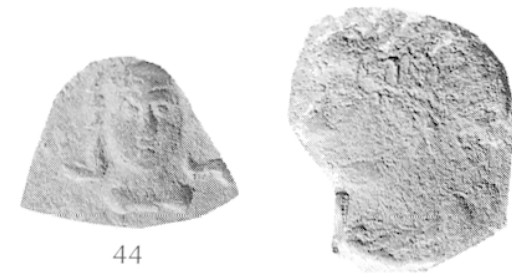

45

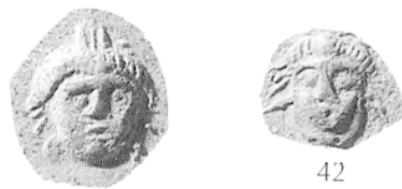

41

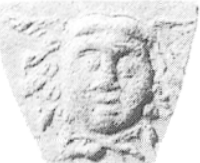

43

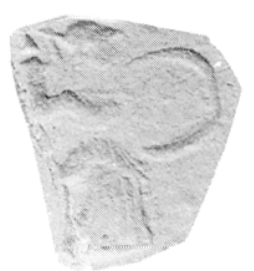

48

47

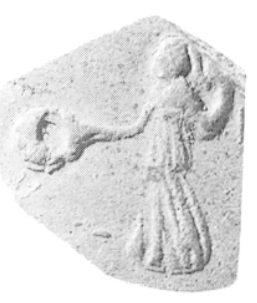

52

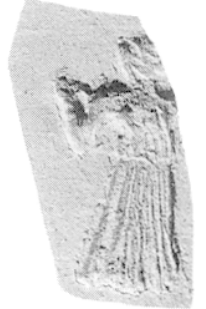

53

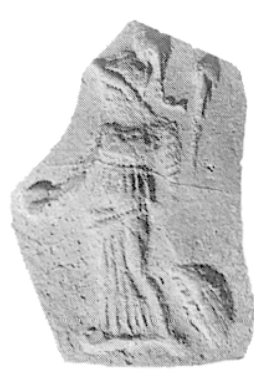

46
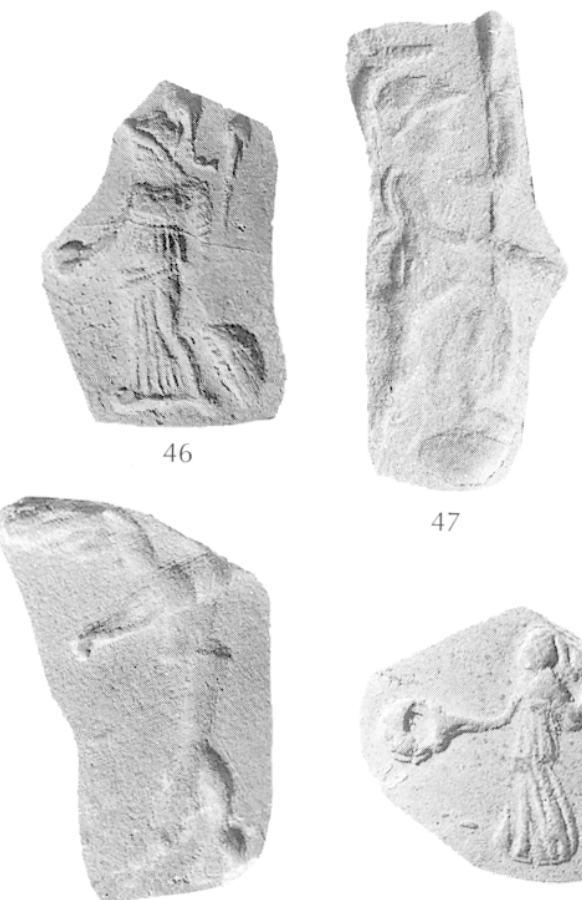

51

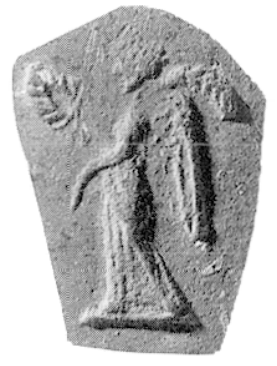

49

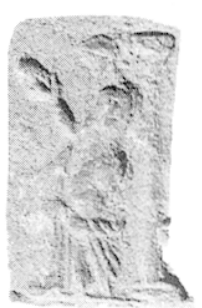

54
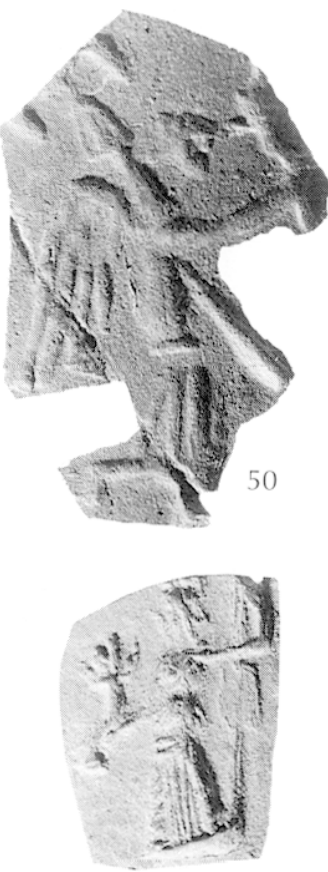

55

Pl. 75. Poinsons: bustes, masques et divinités (échelle : 2/1). 

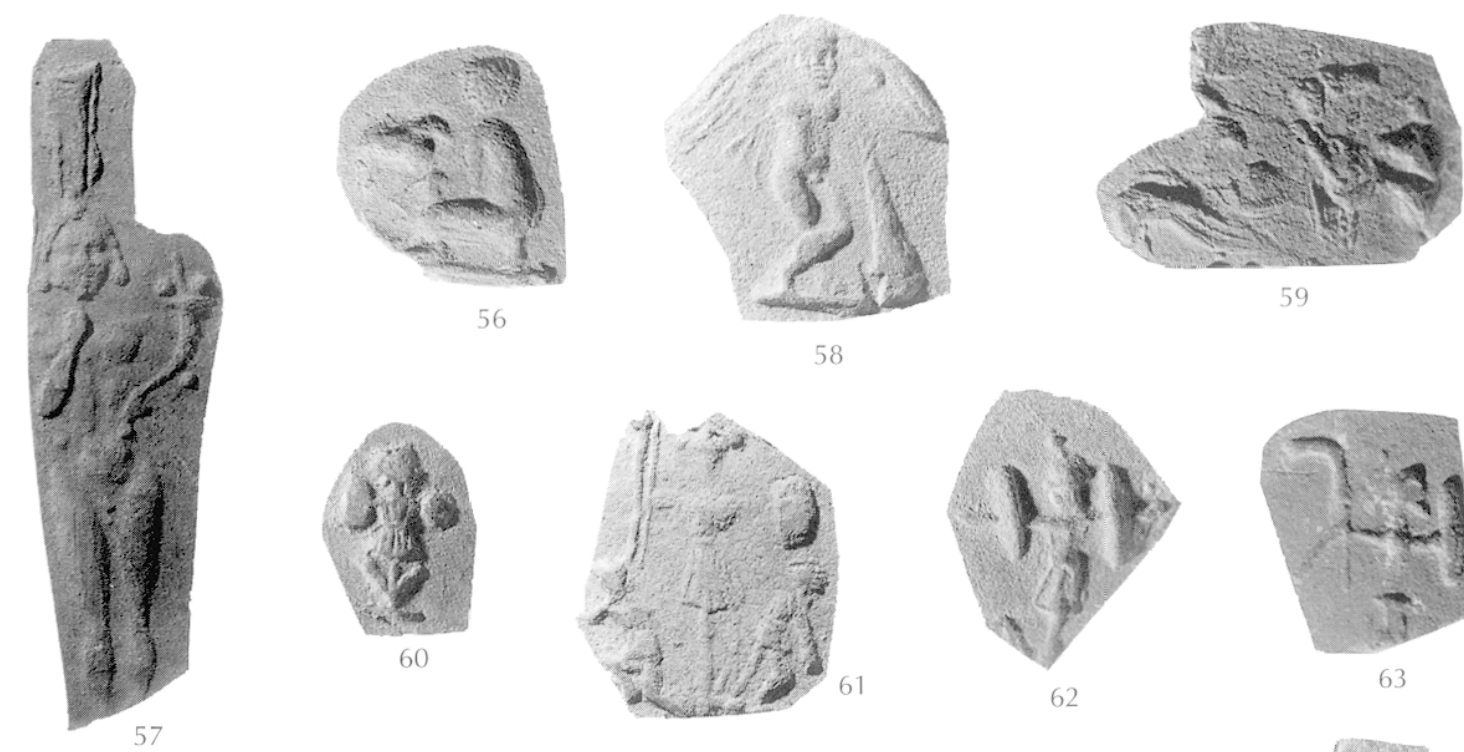

62

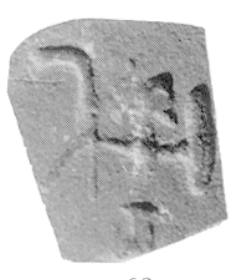

63
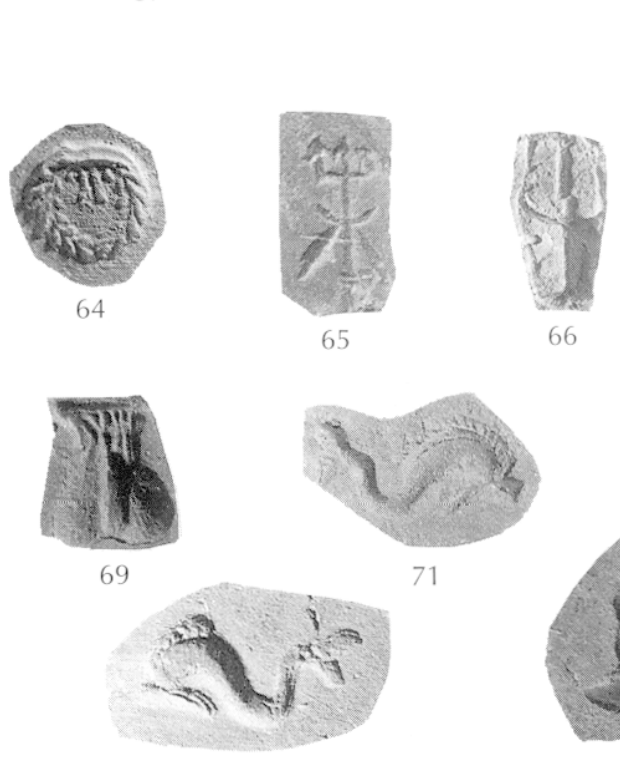

70
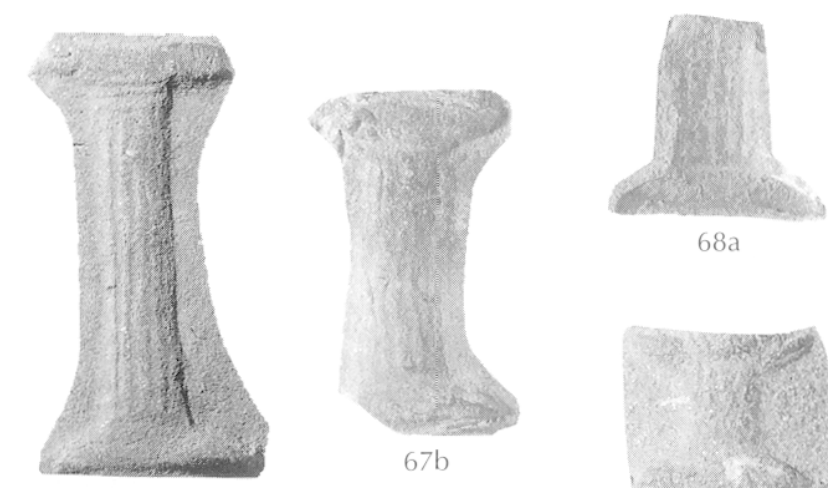

68a
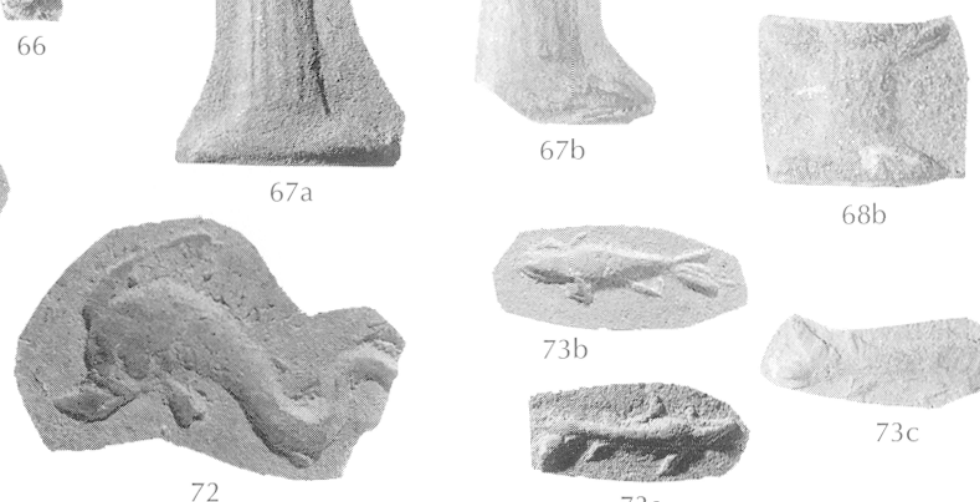

$68 b$
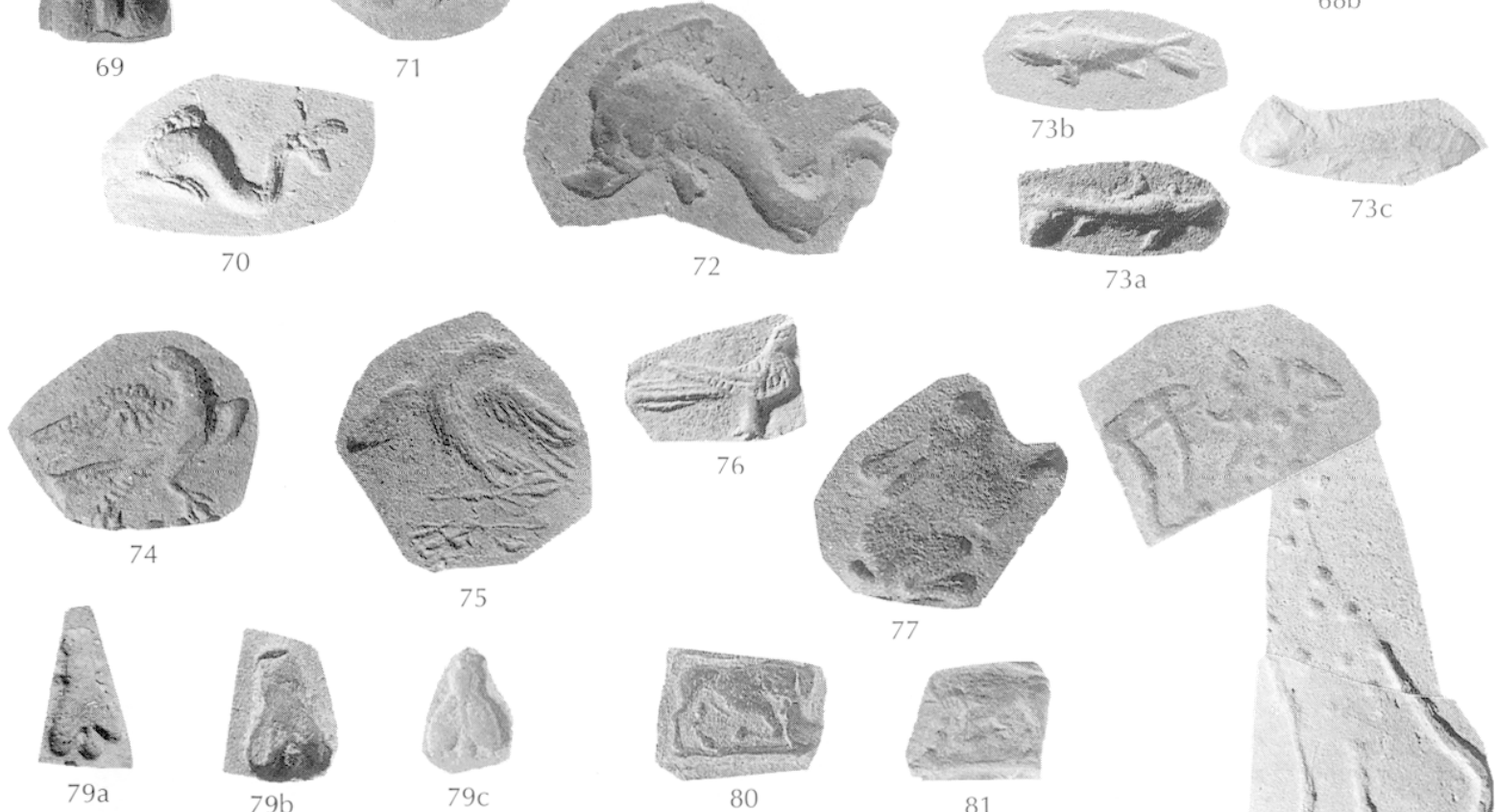

80

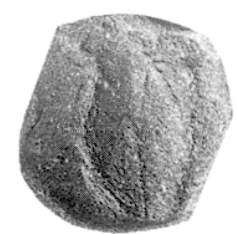

82

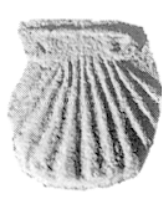

$83 a$

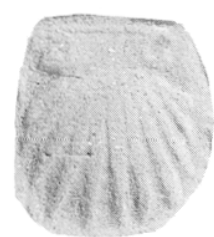

$83 \mathrm{~b}$
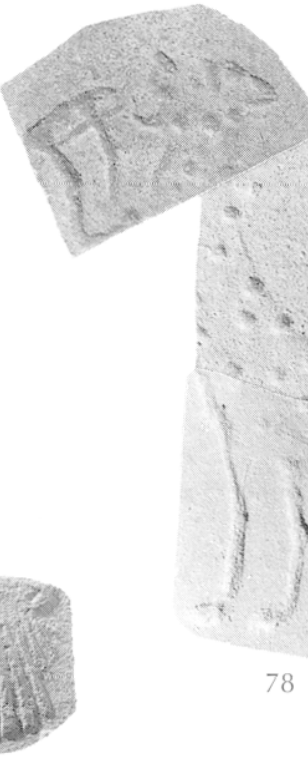

Pl. 76. Poinsons : personnages, attributs et animaux (échelle : 2/1). 

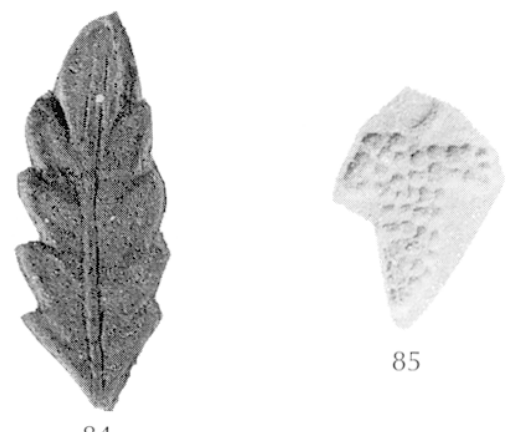

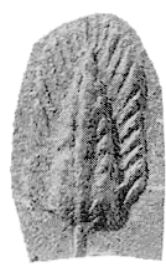

86

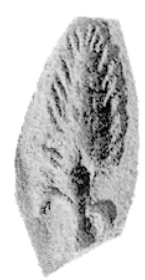

87

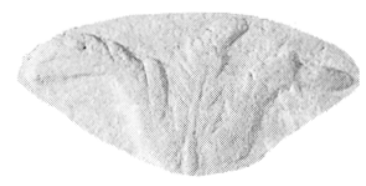

88

85

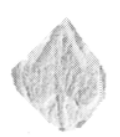

90

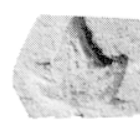

$91 \mathrm{a}$
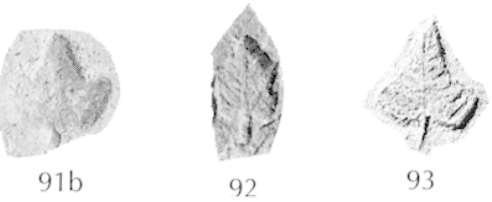

93

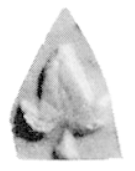

94
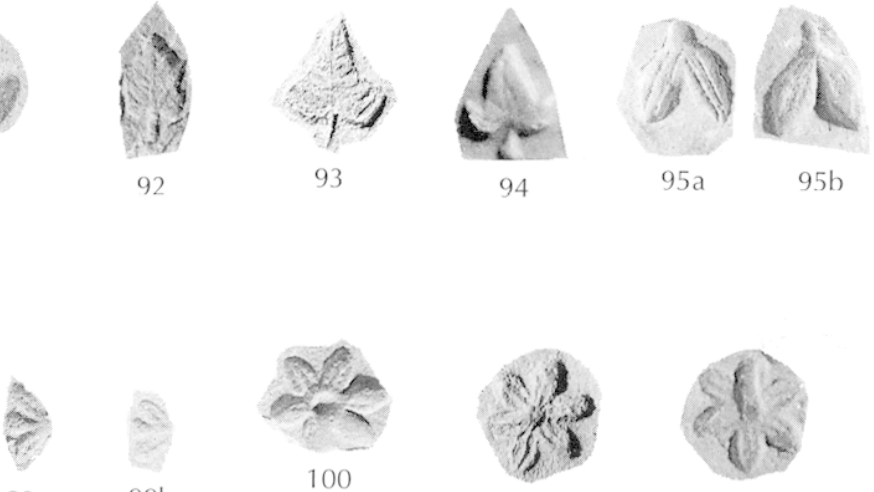

102

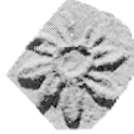

103

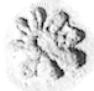

105

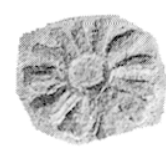

106

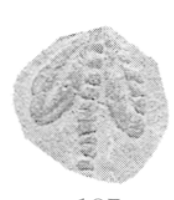

107

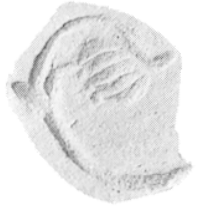

111

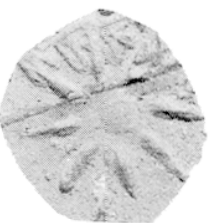

116

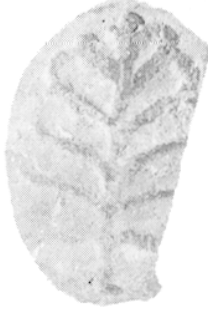

108
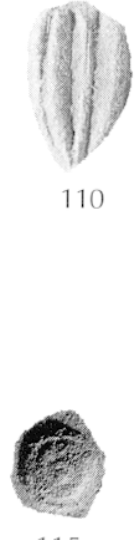

115

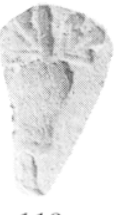

112

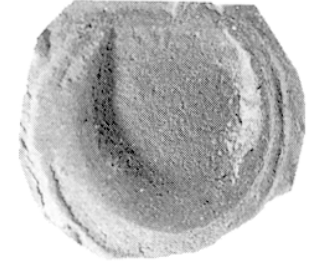

117

P1. 77. Poincons : motifs floraux et divers (échelle : 2/1). 

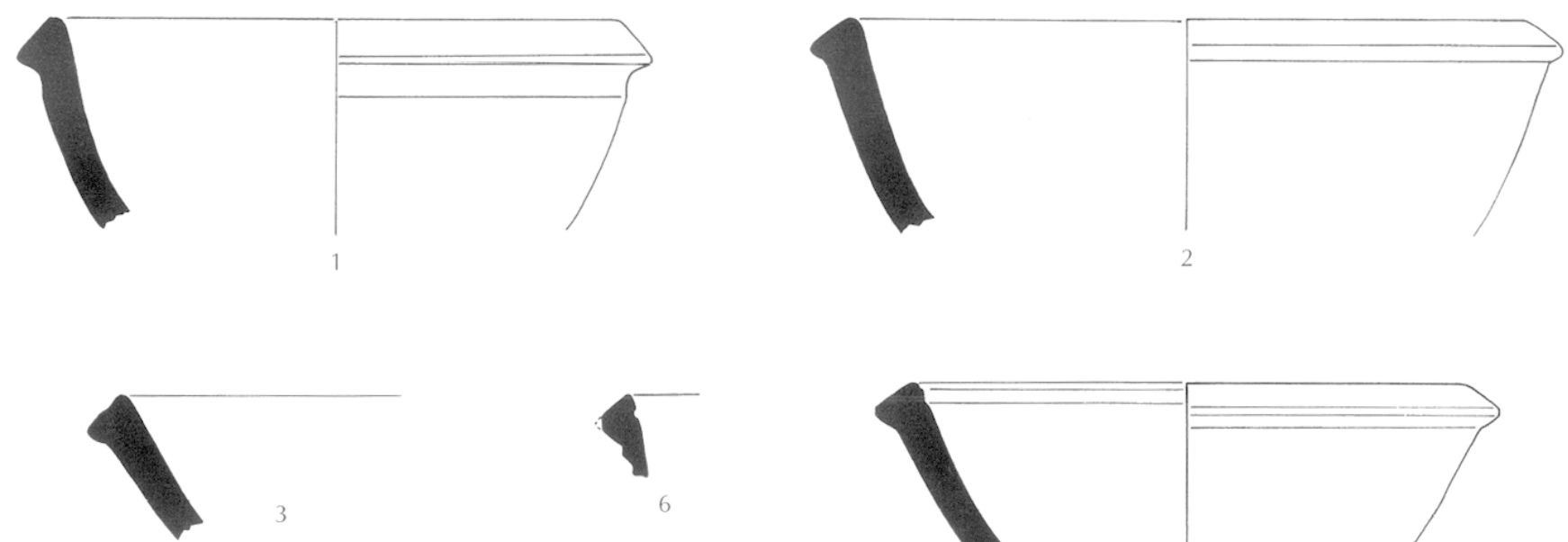

6
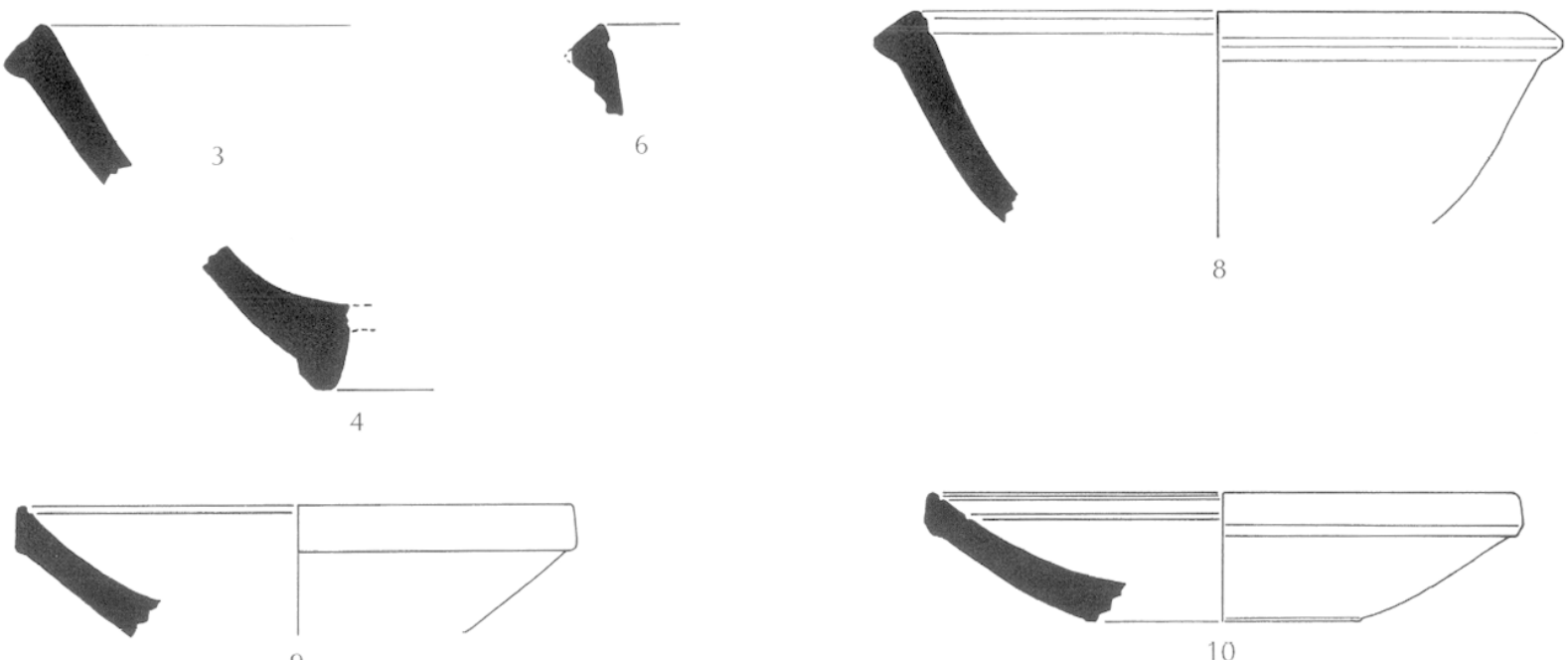

10
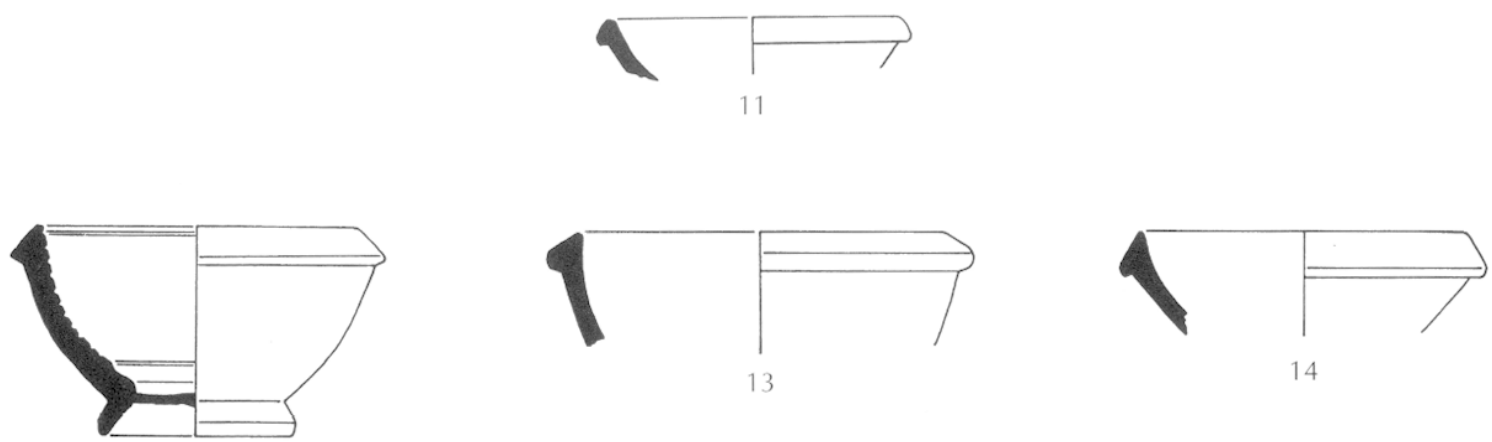

12
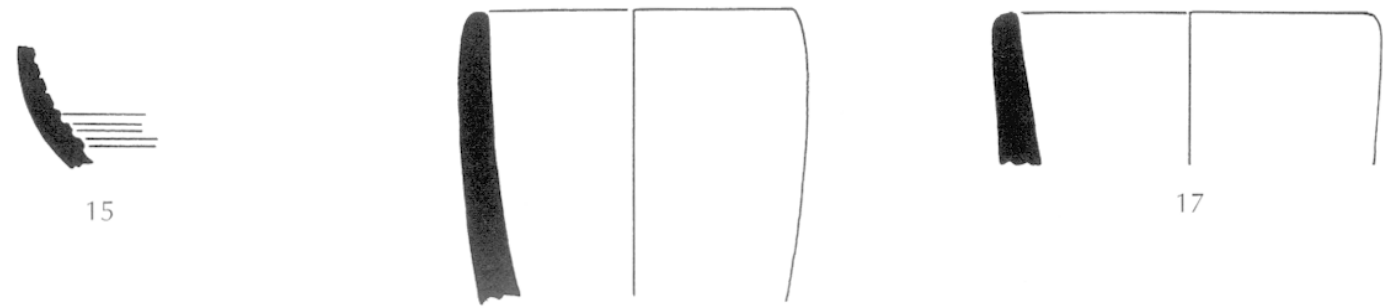

15

17

16

Pl. 78. Moules de sigillée et de gobelets, profils (échelle: 1/3). 

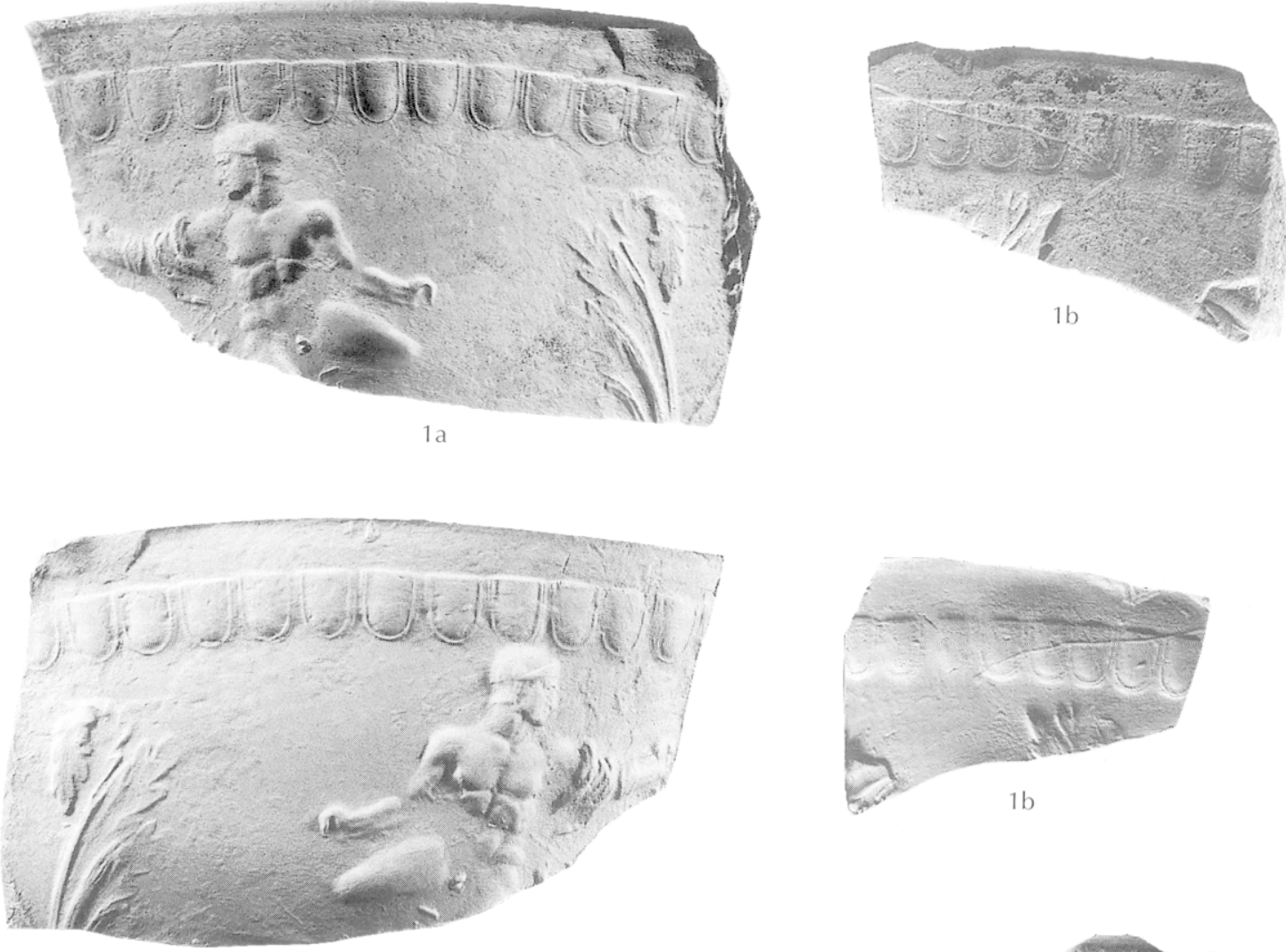

$1 \mathrm{a}$

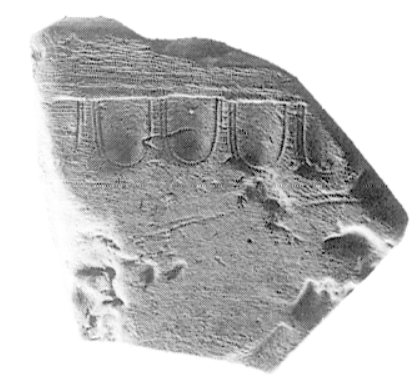

3
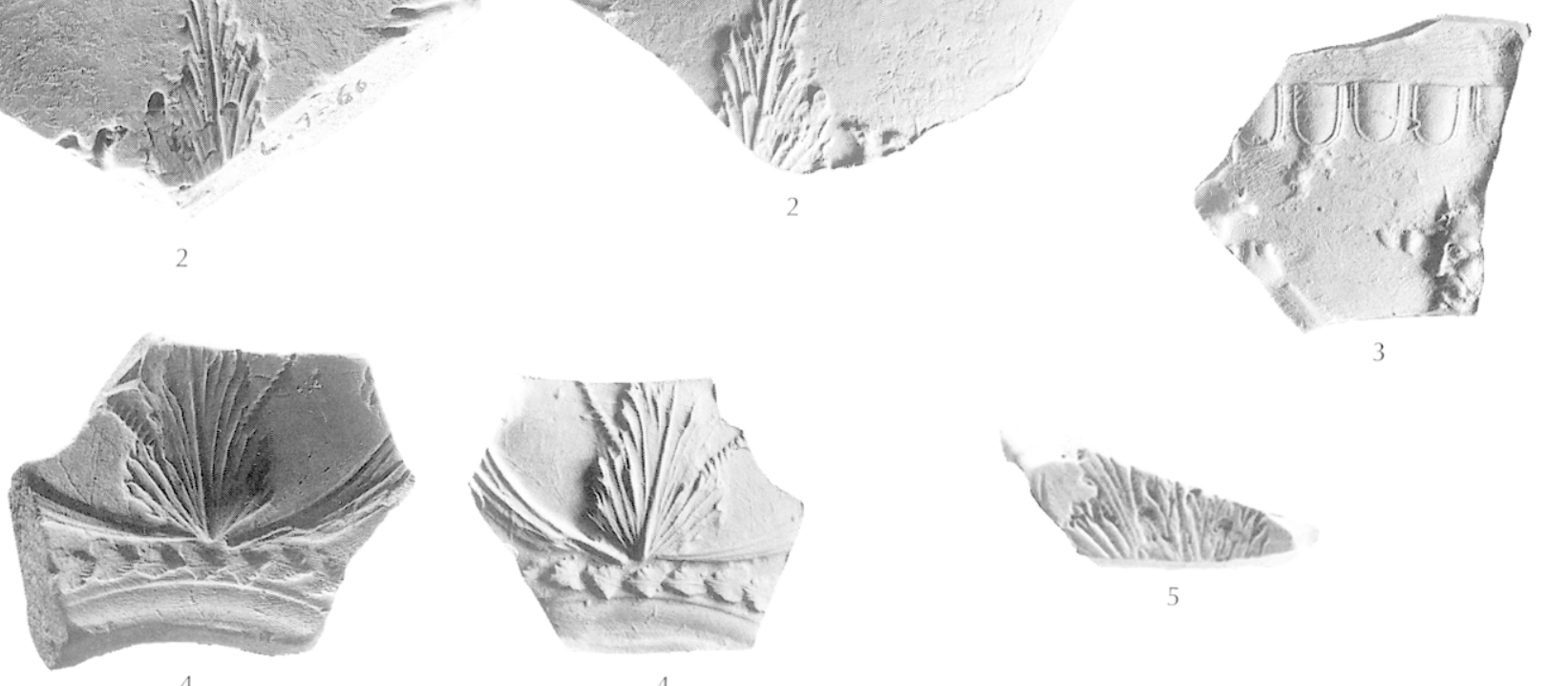

5

Pl. 79. Moules de sigillée (I-5) (échelle : 2/3). 

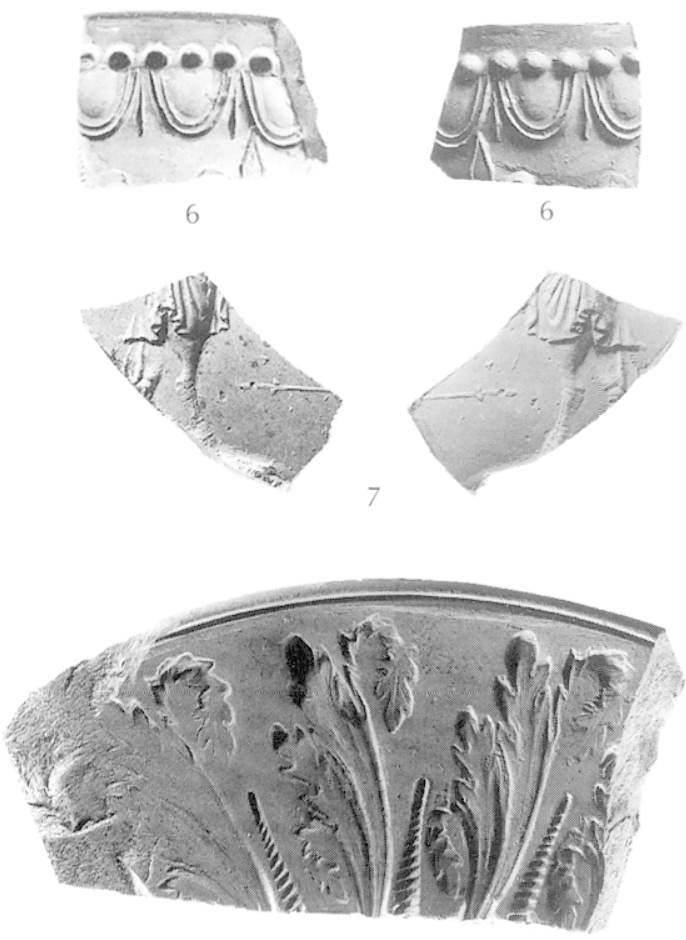

9

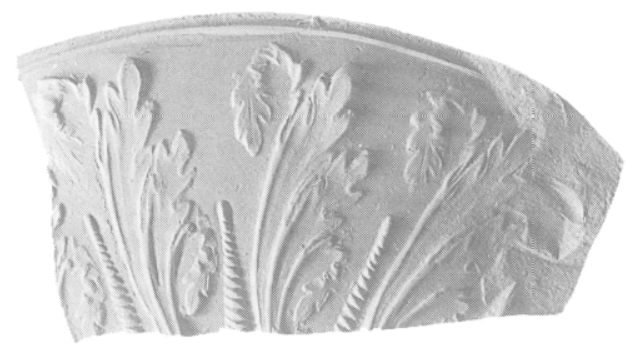

9

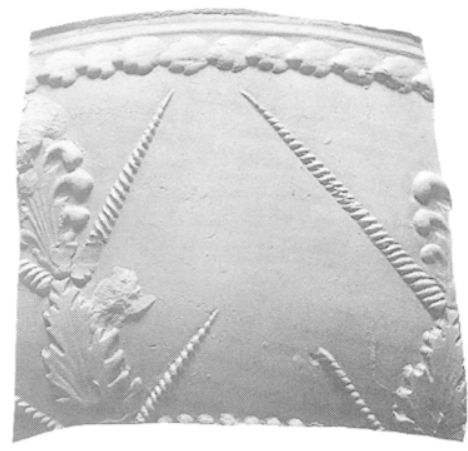

8
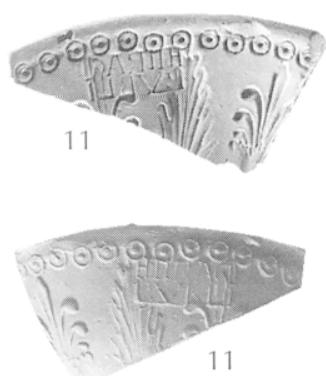
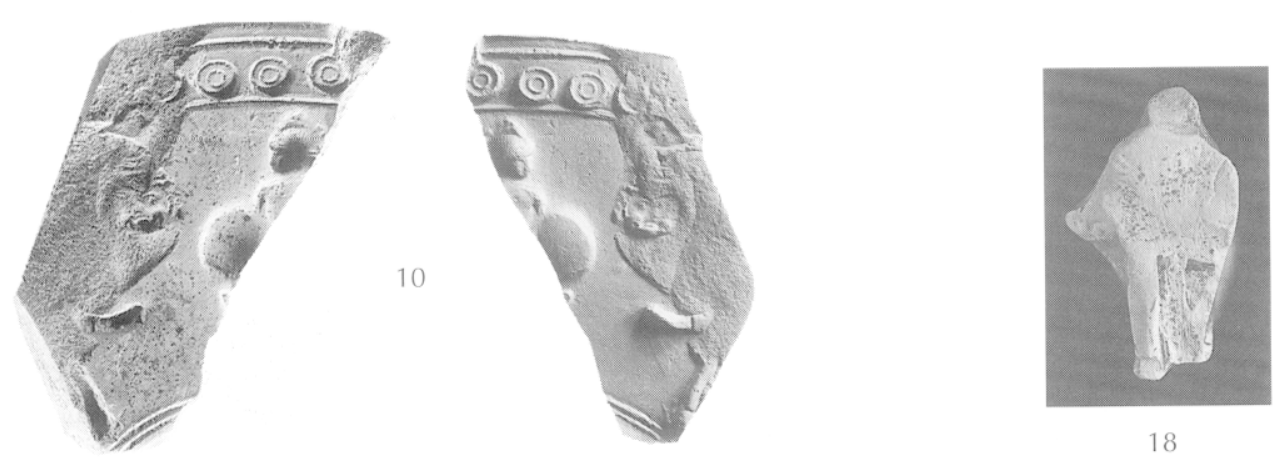

18
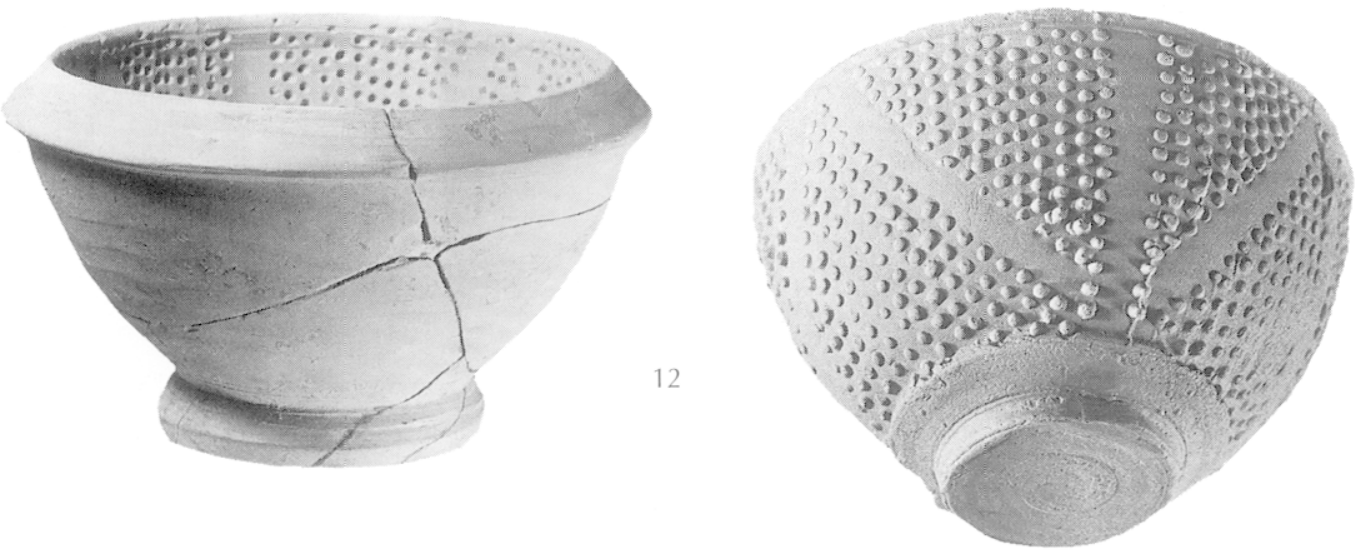

Pl. 80. Moules de sigillée (6-11), poinçon matrice (18) et moule de gobelet (12) (échelle: 2/3). 

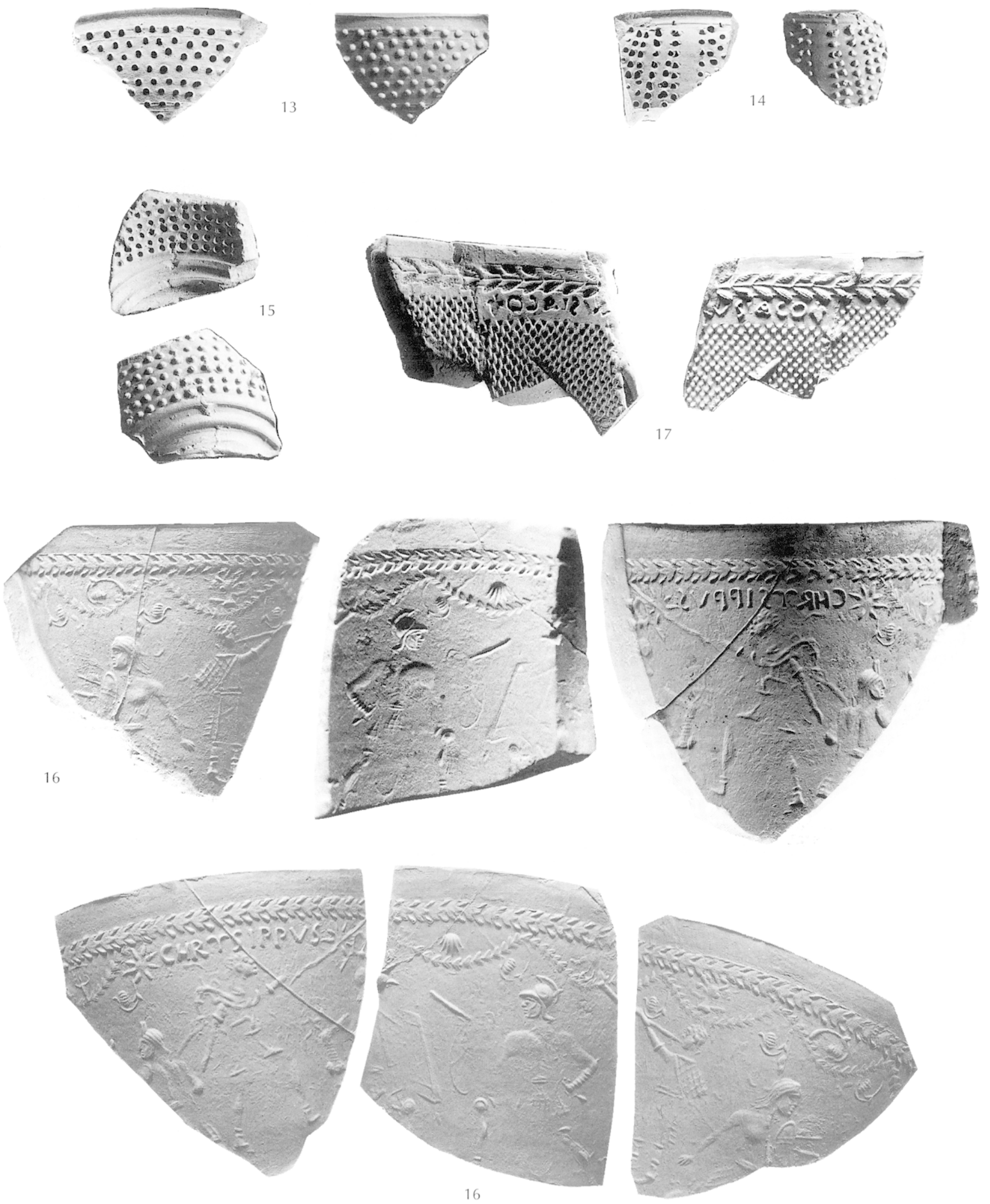

P1. 81. Moules de gobelets à décors de picots (13-15) et moules de gobelets d'Aco (16-17) (échelle : 2/3). 


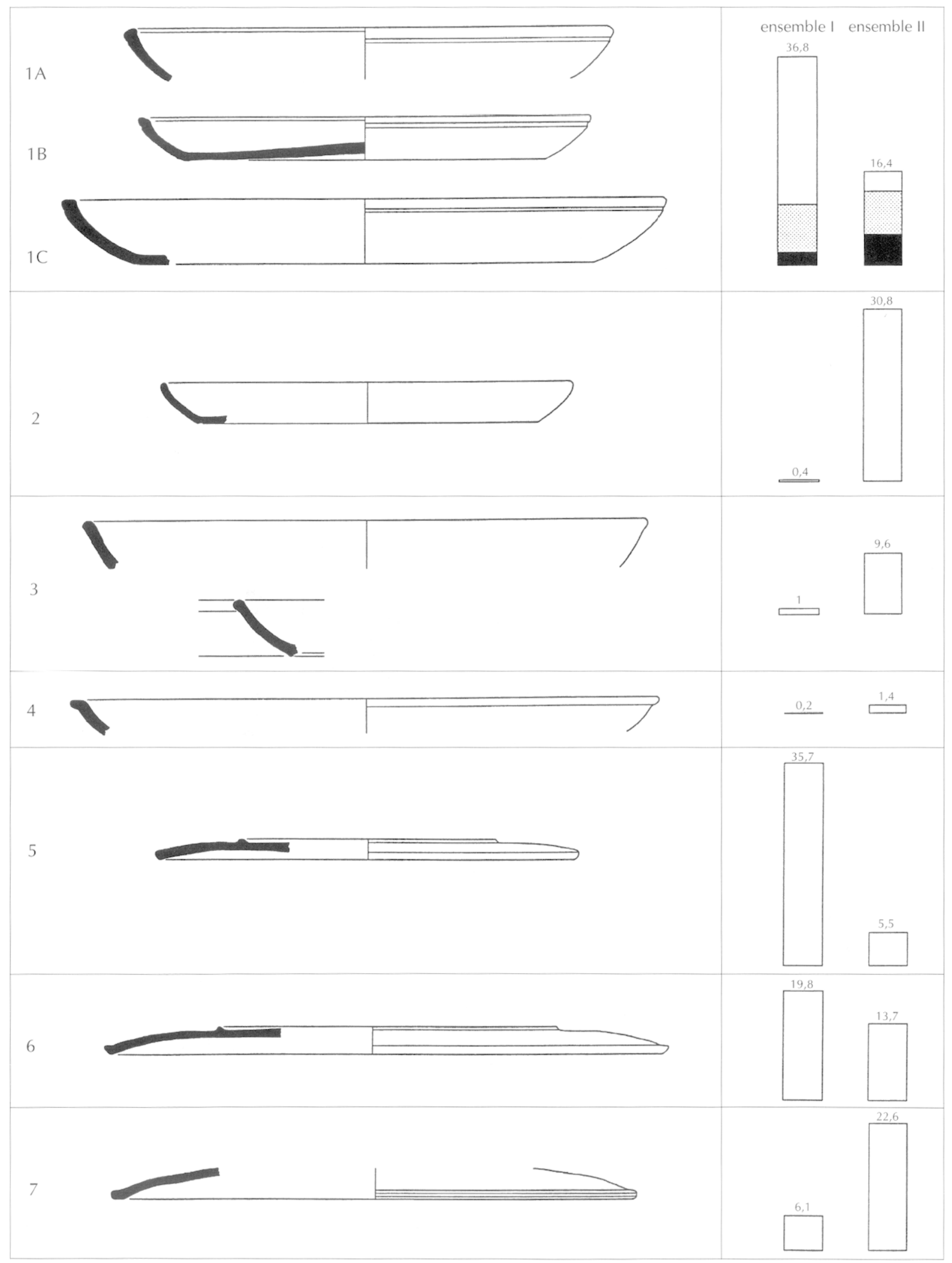

P1. 82. Typologie des plats et des couvercles de céramique à engobe interne rouge pompéien de l'atelier de la Muette et histogrammes de la répartition des types en pourcentage dans chaque ensemble (cf. tabl. VII et VIII pour la quantification chiffrée) (échelle 1/3). 


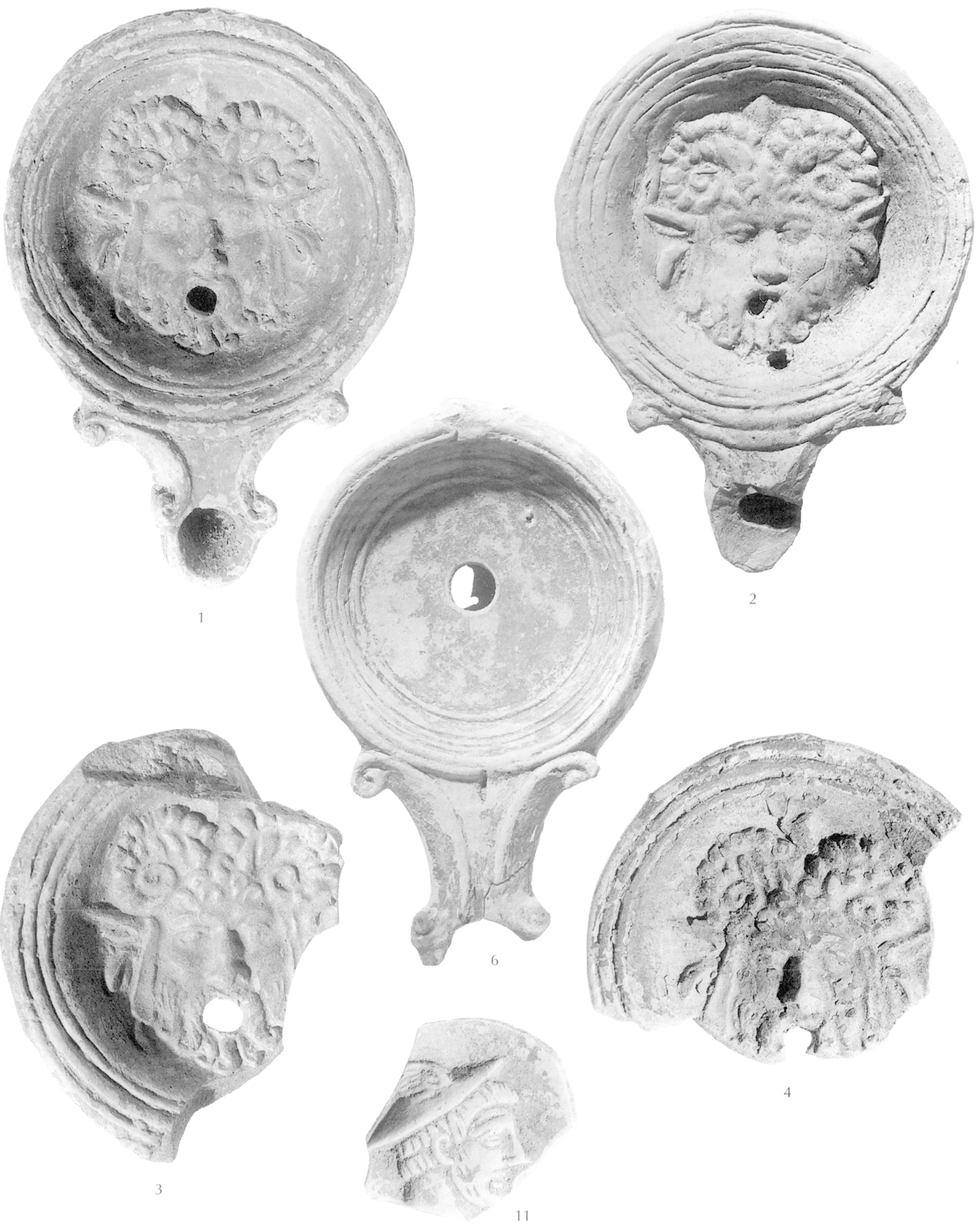

P1. 83. Lampes à huile de l'atelier de la Muette : 1-4, type Muette I; 6, type Muette II; 11, hors typologie (échelle : 1/1). 

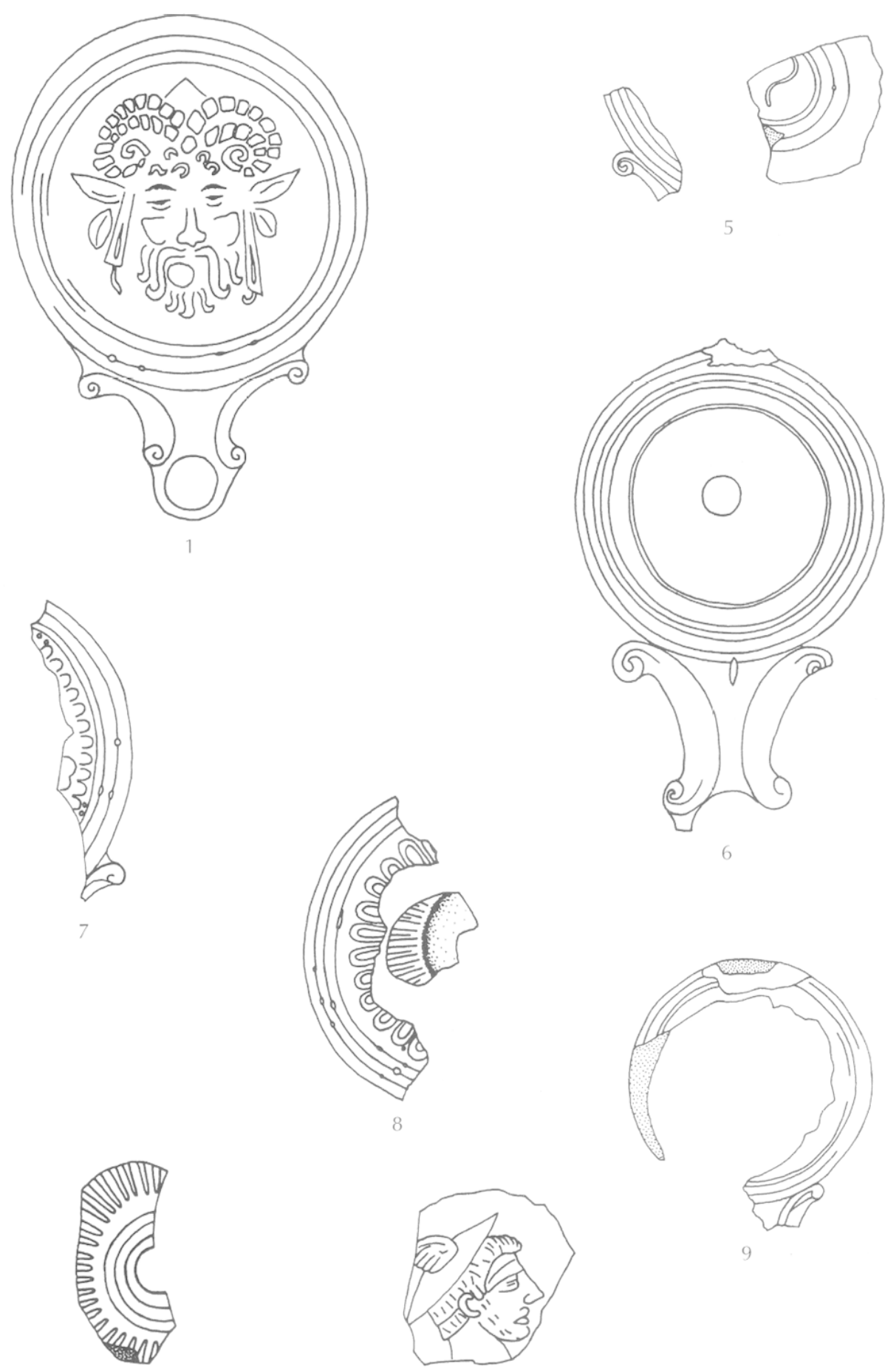

10

11

P1. 84. Lampes à huile de latelier de la .Muette: 1, 5, tye .Muette I; 6, type Muette II; 7, 8. type . Wuette IIIA, ; 9. type . Muetle IIIB: 10, II. hors typologie (échelle : 2/3). 

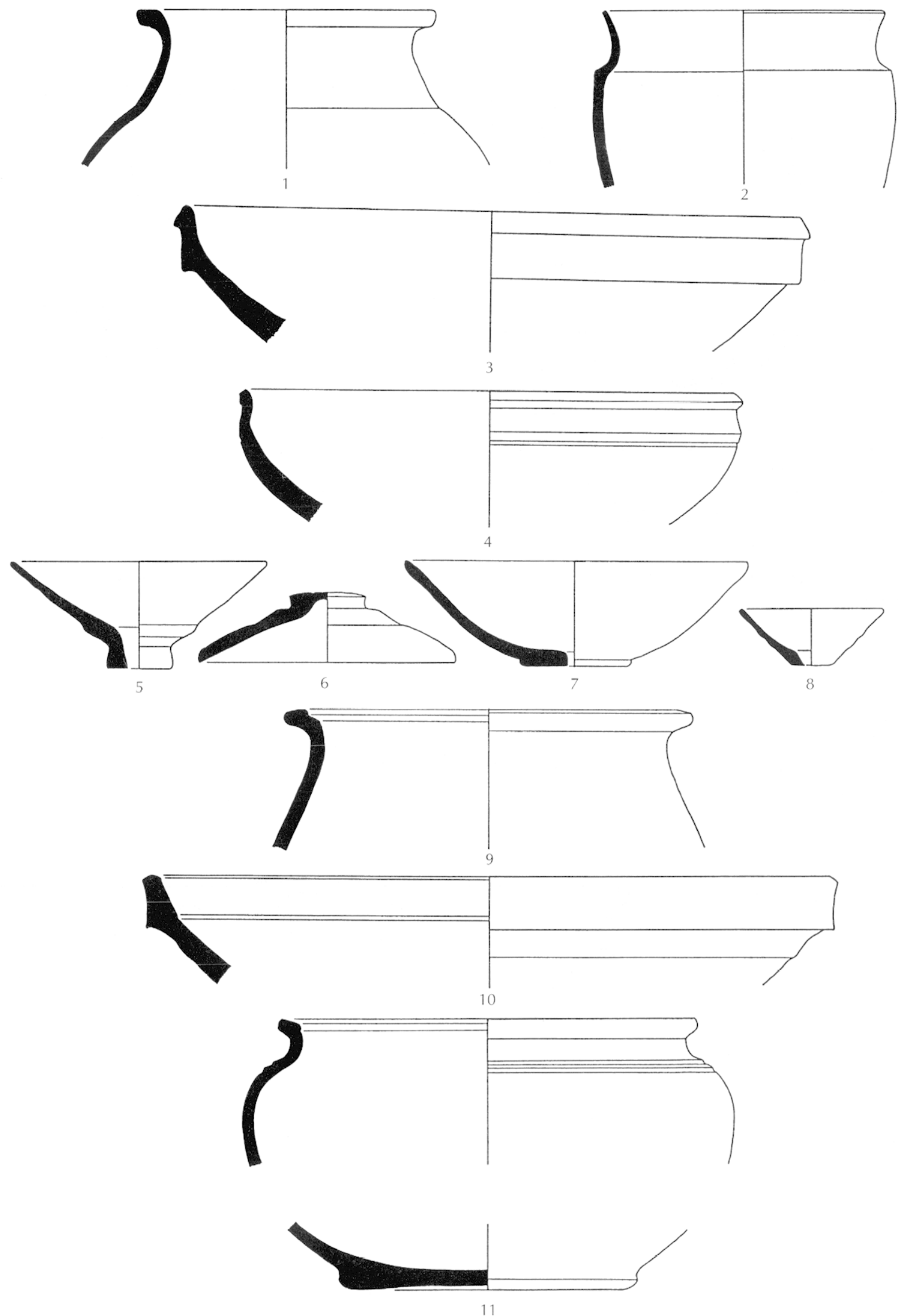

P1. 85. Céramique commune claire de l'ensemble I (échelle: I/3). 

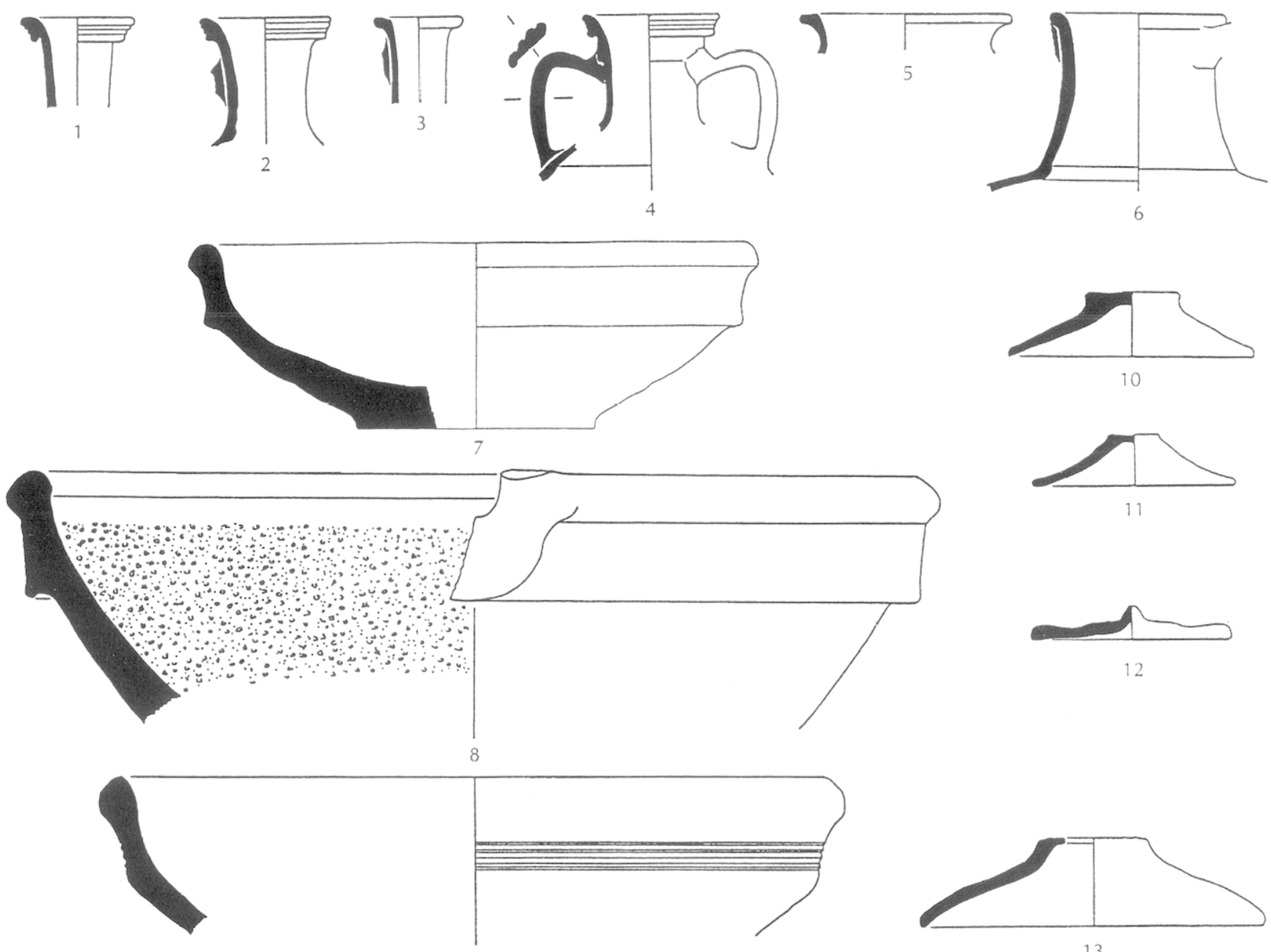

9
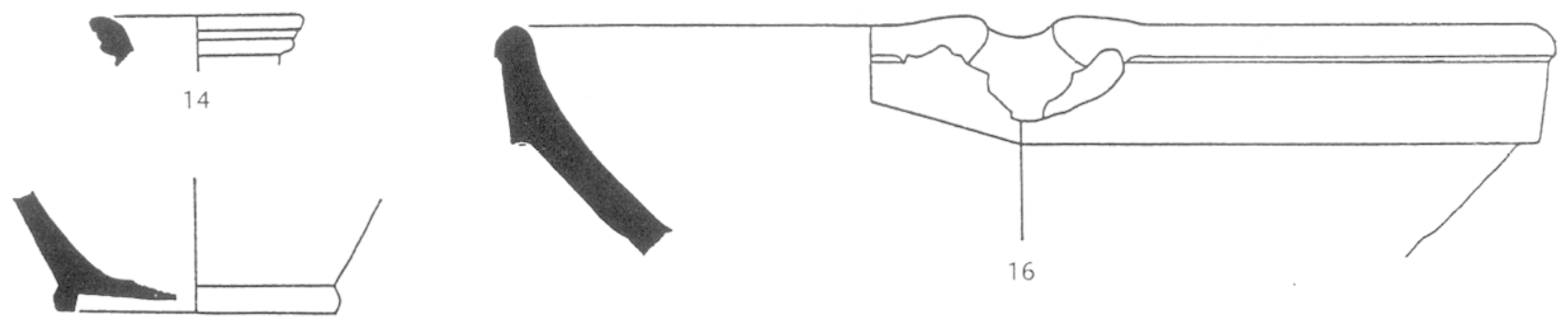

15
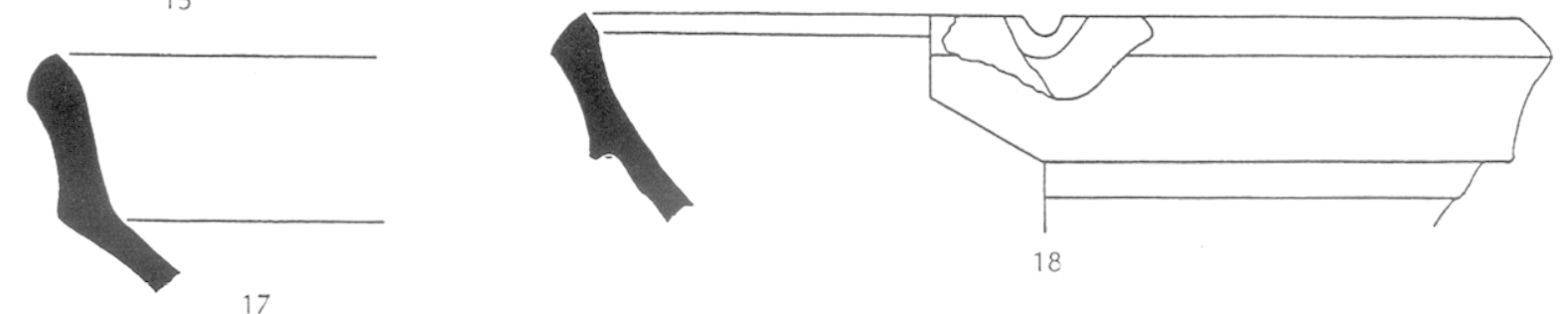

Pl. 86. Céramique commune claire de l'ensemble II léchelle: $1 / 3$ ). 

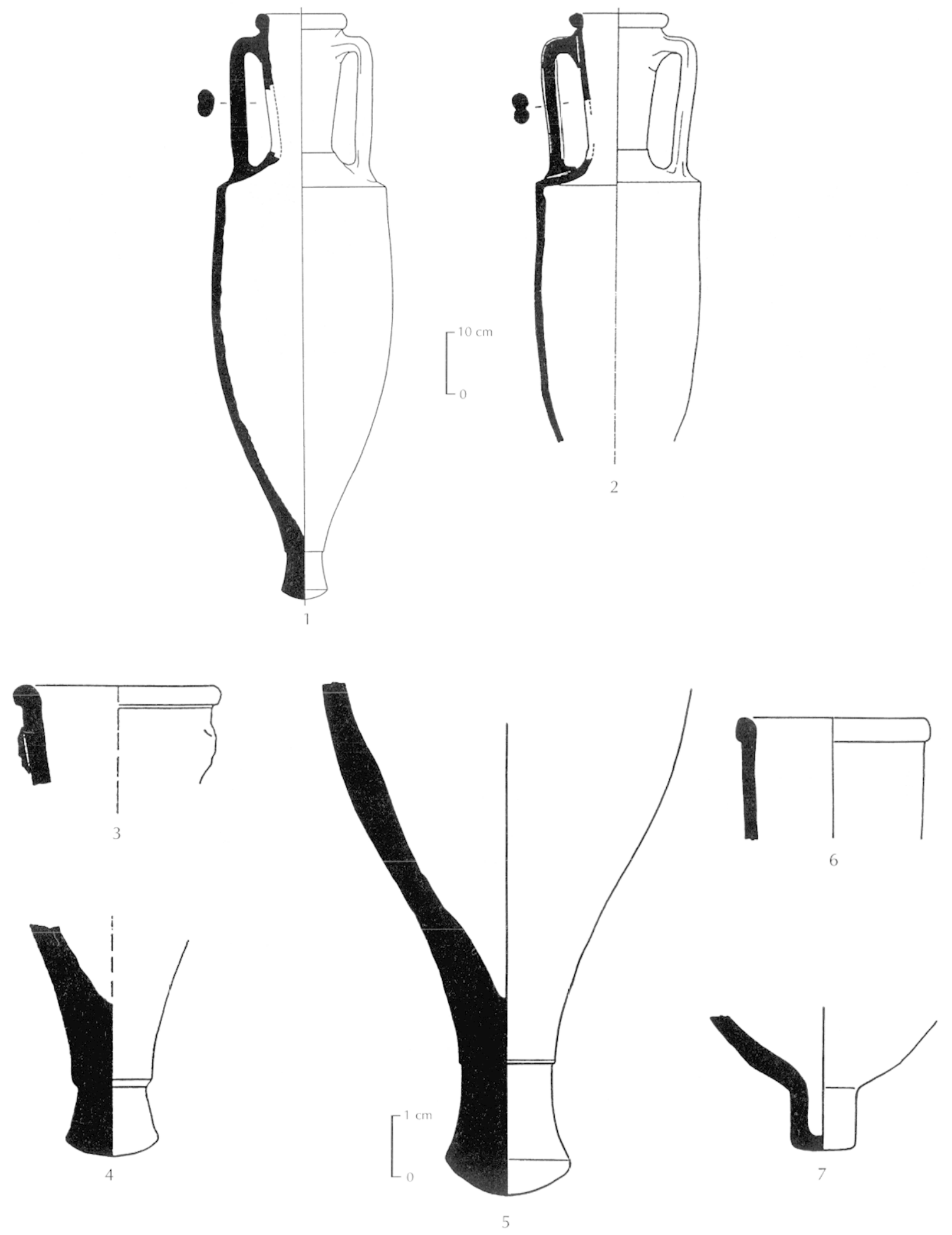

Pl. 87. Amphores Dressel 2/4, type Lyon 2 (échelle : 1/4). 

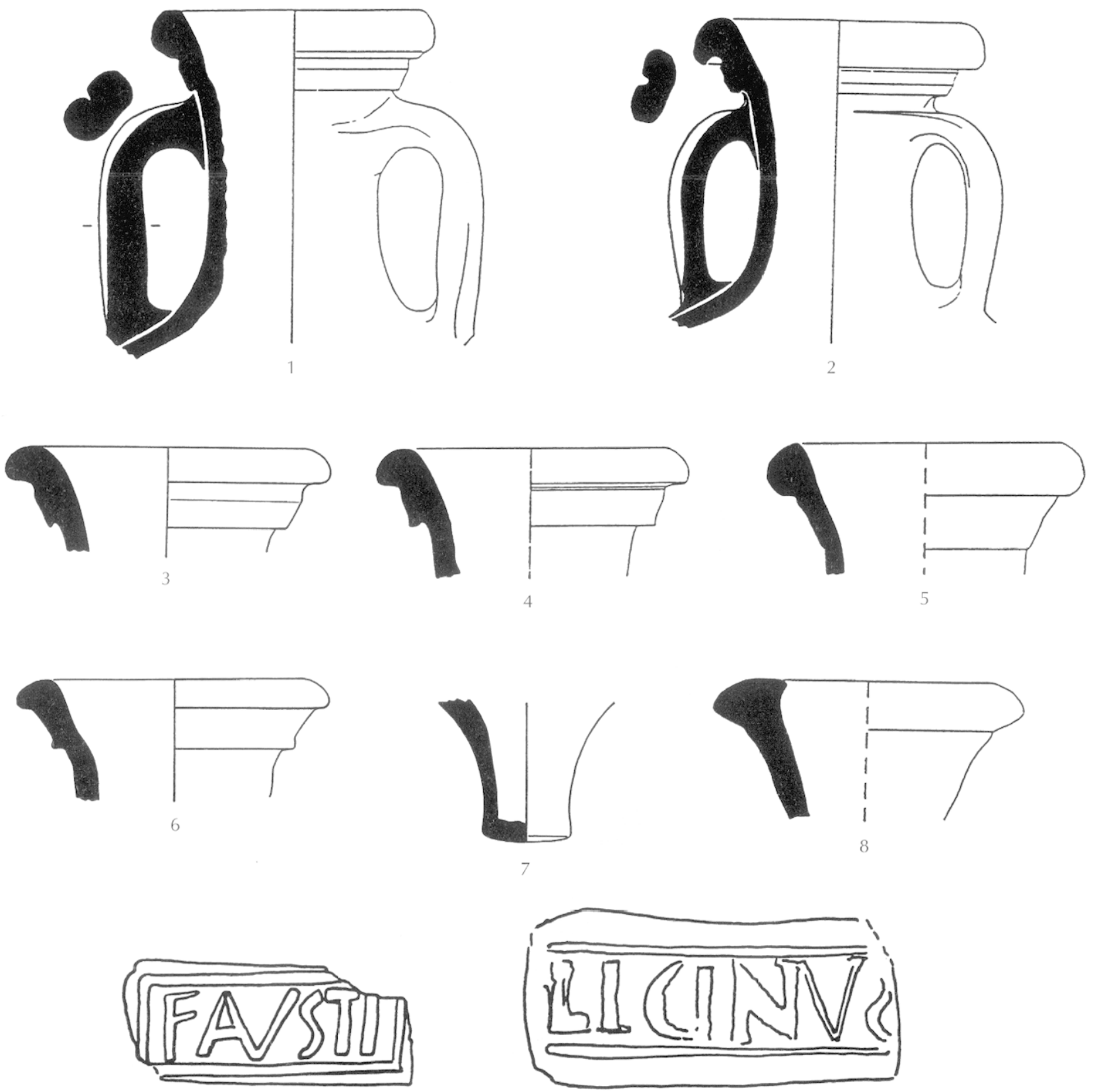

10

P1. 88. Amphores Dressel 9 similis, type Lyon 3 (échelle: $1 / 4$ sauf 9 et $10: 1 / 1$ ). 